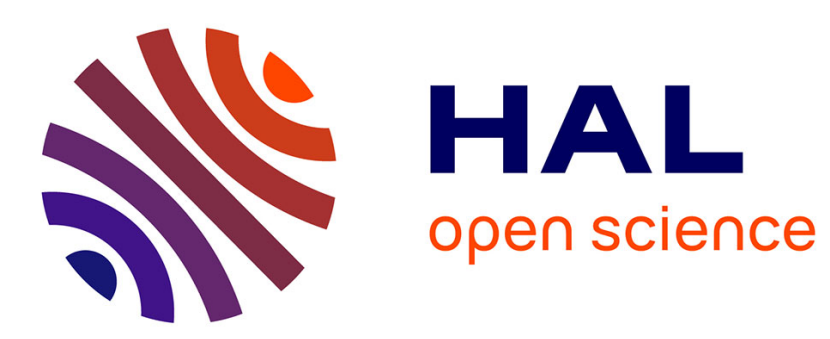

\title{
Mesure d'indépendance linéaire de logarithmes dans un groupe algébrique commutatif
}

\author{
Eric Gaudron
}

\section{To cite this version:}

Eric Gaudron. Mesure d'indépendance linéaire de logarithmes dans un groupe algébrique commutatif. Mathématiques [math]. Université Jean Monnet - Saint-Etienne, 2001. Français. NNT: . tel00001165

\section{HAL Id: tel-00001165 \\ https://theses.hal.science/tel-00001165}

Submitted on 27 Feb 2002

HAL is a multi-disciplinary open access archive for the deposit and dissemination of scientific research documents, whether they are published or not. The documents may come from teaching and research institutions in France or abroad, or from public or private research centers.
L'archive ouverte pluridisciplinaire HAL, est destinée au dépôt et à la diffusion de documents scientifiques de niveau recherche, publiés ou non, émanant des établissements d'enseignement et de recherche français ou étrangers, des laboratoires publics ou privés. 


\title{
Mesure d'indépendance linéaire de logarithmes dans un groupe algébrique commutatif
}

\author{
THÈSE \\ présentée et soutenue publiquement le 8 décembre 2001 \\ pour l'obtention du grade de
Docteur de l'Université Jean Monnet — Saint-Étienne (spécialité Mathématiques) \\ par \\ Éric Gaudron
}

Composition du jury

$\begin{array}{llll}\text { Président: } & \text { M. Waldschmidt } & \text { Professeur } & \text { Université Paris VI } \\ \text { Directeur : } & \text { G. Diaz } & \text { Professeur } & \text { Université Jean Monnet } \\ \text { Rapporteurs : } & \text { J.-B. Bost } & \text { Professeur Université Paris XI } \\ & \text { N. Hirata-Kohno } & \text { Professeur Université Nihon (Tokyo) } \\ \text { Examinateurs : } & \text { S. David } & \text { Maître de Conférences Université Paris VI } \\ & \text { M. Laurent } & \text { Directeur de Recherches C.N.R.S }\end{array}$





\section{Remerciements}

De nombreuses personnes ont contribué, de près ou de loin, à l'élaboration de ce travail. Mais c'est en premier lieu à S. David et M. Waldschmidt que je tiens à exprimer mes plus vifs remerciements. Leur patience à mon égard, leur disponibilité, leur aide constante et soutenue (en dépit de mon éloignement géographique) m’ont permis de mener à bien ces recherches. Ils m'ont transmis une vision claire et moderne de l'Approximation Diophantienne.

Ma reconnaissance s'adresse également à G. Diaz qui a toujours exprimé un très vif intérêt pour mes travaux. Son suivi permanent, les nombreuses discussions que j'ai eues avec lui et le travail important qu'il a fourni en lisant et en corrigeant les innombrables versions de ma thèse m'ont été d'une aide précieuse, dont je mesure le prix.

Je remercie N. Hirata-Kohno d'avoir accepté de rapporter ma thèse et de s'être acquittée de cette tâche avec promptitude et minutie, et, qui plus est, m'a fait l'immense honneur de venir du Japon pour être présente le jour de ma soutenance. Je remercie également J.-B. Bost dont les nombreuses remarques et suggestions me seront très utiles pour rendre plus accessibles plusieurs points de ce travail. Je suis particulièrement touché de l'acuité avec laquelle il a situé - au milieu de calculs (parfois assez lourds) qui jonchent la seconde partie de ce travail - les points délicats en me signalant quelques imprécisions et inexactitudes. Les cours que J.-B. Bost a donné à l'Institut Henri Poincaré en 1997 et 1999 ont été déterminants pour la seconde partie de ma thèse et resteront pour moi un très grand souvenir.

Je remercie encore M. Laurent d'avoir accepté de participer à mon jury, ainsi que D. Bertrand, P. Graftieaux et P. Philippon avec lesquels j'ai eu des conversations très instructives (voire décisives).

Au cours de mon séjour à Saint-Étienne, j'ai bénéficié du soutien amical et attentif de tous les membres du LArAl*, et, en particulier, de N. Brisebarre et F. Gramain qui ont joué un rôle notable dans le bon déroulement de ce travail. Le soutien du LArAl ne s'est pas limité aux encouragements d'usage vis à vis d'un thésard. En fait, les conditions matérielles et humaines que l'on m'a offertes ont été extraordinaires, avec, ce qui me tient le plus à cœur, la possibilité d'assister à de nombreuses conférences et colloques en France et à l'étranger.

Pour tout cela, je vous dis un grand merci à tous !

*Laboratoire d'Arithmétique et d'Algèbre (de Saint-Étienne). 


\section{Table des matières}

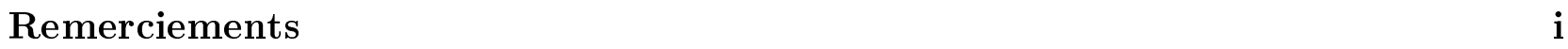

Notations et conventions générales $\quad$ ix

Introduction $\quad$ xi

Mesure d'indépendance linéaire de logarithmes I

Le cas général

$1 \quad$ Introduction . . . . . . . . . . . . . . . . . . 3

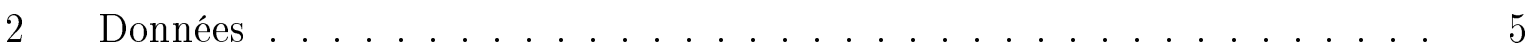

2.a Le groupe algébrique ................. 5

2.b Données arithmétiques . . . . . . . . . . . 6

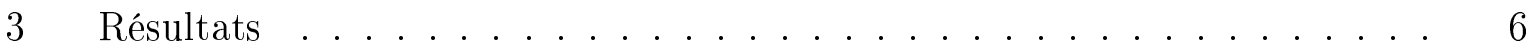

3.a Énoncés ........................... 6

3.b Commentaires et conséquences ............ 8

3.c Cas d'un groupe linéaire commutatif . . . . . . . . . . . . 11

$4 \quad$ Organisation du texte . . . . . . . . . . . . . . . 14

$5 \quad$ Modèle du groupe algébrique . . . . . . . . . . . . . . . . . . . 14

6 Réductions . . . . . . . . . . . . . . . . . . 17

$7 \quad$ Choix des paramètres f..................... 18 
8 Choix de bases pour l'hyperplan $W \ldots \ldots \ldots \ldots \ldots \ldots$

9 Le cas périodique : compléments . . . . . . . . . . . . 23

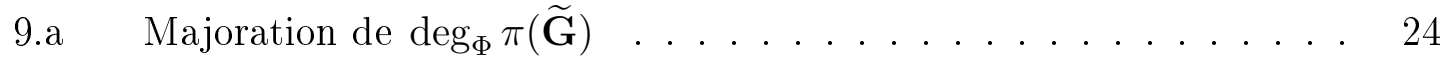

9.b Nouvelle base pour $W \otimes \mathbb{C} \ldots \ldots \ldots . \ldots . \ldots . \ldots 25$

10 Changement de bases de dérivations . . . . . . . . . . . . 26

11 Rang du système linéaire . . . . . . . . . . . . . . . . . . . . 27

12 Construction de la fonction auxiliaire . . . . . . . . . . . . . . 29

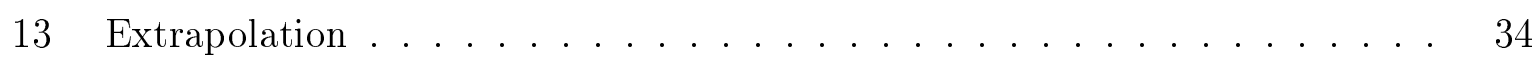

14 Estimations ultramétriques . . . . . . . . . . . . . . 37

15 Estimations archimédiennes . . . . . . . . . . . . . . . . 42

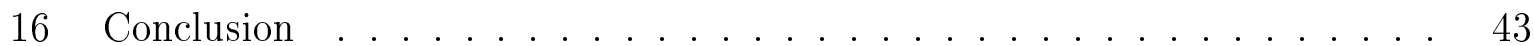

$\begin{array}{ll}\text { A Paramètres libres } & 45\end{array}$

A.1 Les paramètres . . . . . . . . . . . . . . . . . . 45

A.2 Le cas périodique : compléments . . . . . . . . . . . 47

A.3 Rang du système linéaire . . . . . . . . . . . . . . . . . . . . . . . . 48

A.4 Construction de la fonction auxiliaire . . . . . . . . . . . . . . . . 48

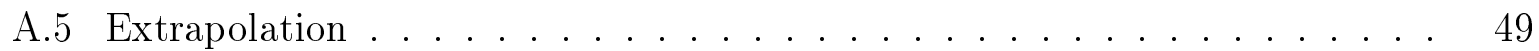

A.6 Estimations ultramétriques et archimédiennes . . . . . . . . . . 50

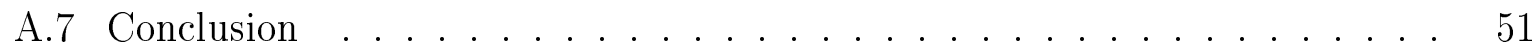

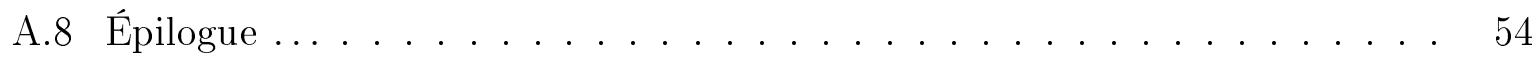

A.8.a Les protagonistes . . . . . . . . . . . . . . 54

A.8.b Tableau des contraintes .................. 56

A.8.c Justification du choix des paramètres . . . . . . . . . . . . . 57 


\section{Le cas abélien non-homogène}

$1 \quad$ Introduction . . . . . . . . . . . . . . . . . . . 61

2 Données et résultats . . . . . . . . . . . . . . . 62

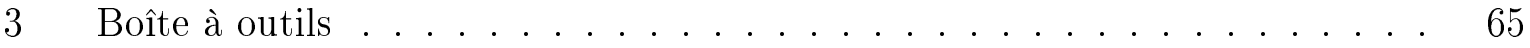

3.a Modèles de Moret-Bailly . . . . . . . . . . . . . 66

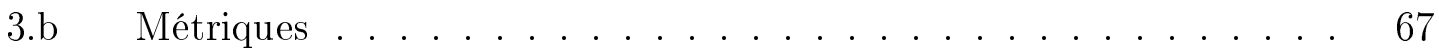

Métrique de Fubini-Study . . . . . . . . . . . . . . . 67

Métrique sur l'espace tangent $t_{\mathbf{G}_{\sigma}}(\mathbb{C}) \ldots \ldots . \ldots . \ldots 69$

Métrique sur la puissance symétrique . . . . . . . . . . . 69

Métrique sur un fibré inversible d'une variété abélienne complexe . 70

3.c Quelques résultats auxiliaires ............... 71

Irréductibilité d'une action de groupe . . . . . . . . . . 71

Groupe unitaire . . . . . . . . . . . . . . . 72

Un calcul de hauteur . . . . . . . . . . . . 73

3.d Méthode des pentes . . . . . . . . . . 74

4 Schéma tactique de la preuve . . . . . . . . . . . 75

5 Démonstration du théorème . . . . . . . . . . . . 76

5.a Choix des paramètres, du fibré hermitien de départ et de la filtration 76

Liste des paramètres . . . . . . . . . . . . 76

Choix du fibré hermitien . . . . . . . . . . . . 79

Choix de la filtration . . . . . . . . . . . . 81

5.b Pente maximale des fibrés hermitiens quotients . . . . . . . 84

5.c Rang d'un sous-fibré ... . . . . . . . . . . . 85

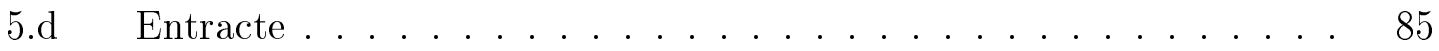

5.e Reprise analytique ................. 86

5.f Hauteur des morphismes . . . . . . . . . . . . 87

Évaluation simple des $\left\|\widetilde{\varphi}_{i}\right\|_{\sigma} \ldots \ldots \ldots . \ldots . \ldots 87$

Estimation fine des $\left\|\widetilde{\varphi}_{i}\right\|_{\sigma_{0}} \ldots \ldots . \ldots . \ldots . . \ldots 90$

Hauteur des morphismes . . . . . . . . . . . 93

5.g Conclusion ............................ 94 
B Inégalités de pentes et Transcendance $\quad 97$

B.1 Définitions . . . . . . . . . . . . . . . . . . . . 97

B.2 Pentes des fibrés vectoriels hermitiens . . . . . . . . . . . . 98

B.3 Pentes pour les morphismes . . . . . . . . . . . . . . . . 98

B.3.a Théorie locale . . . . . . . . . . . . . . 99

B.3.b Théorie globale . . . . . . . . . . . . . . . . . . 99

B.4 Inégalités de pentes . . . . . . . . . . . . . . . . . 100

B.5 Lien avec la transcendance . . . . . . . . . . . . . . . . 101

B.6 Application à la transcendance . . . . . . . . . . . . . . . . . . . . . 102

B.6.a Données . . . . . . . . . . . . . . . . . 102

B.6.b Injectivité de $\varphi_{K} \ldots \ldots \ldots \ldots$. . . . . . . . . . . 103

B.6.c Évaluation de $\left\|\varphi_{K}^{i}\right\|_{\mathfrak{p}},\left\|\varphi_{K}^{i}\right\|_{\sigma} \ldots \ldots \ldots \ldots$

B.6.d Majoration plus fine pour $\left\|\varphi_{K}^{1}\right\|_{\sigma_{0}} \ldots \ldots$. . . . . . . . . 104

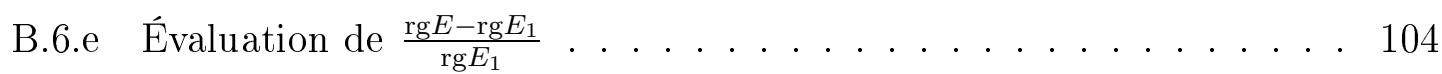

B.6.f On applique l'inégalité de pente . . . . . . . . . . . . . . . 104

B.6.g Conclusion ...................... 105

C Aide-mémoire sur la notion de voisinage infinitésimal 107

C.1 Introduction . . . . . . . . . . . . . . . . . . . 107

C.2 Voisinages infinitésimaux . . . . . . . . . . . . . . 107

C.2.a Schémas et groupes formels . . . . . . . . . . . . . . 109

C.2.b Cas d'un fibré vectoriel . . . . . . . . . . . . . . . . 111

C.2.c Cas d'un schéma en groupes . . . . . . . . . . . . . . . 112

C.3 Notion de lissité . . . . . . . . . . . . . . . . . . . . . . . . . 117

C.4 Exemples de morphismes lisses . . . . . . . . . . . . . . . . . . 119

C.4.a Fibrés vectoriels et fibrés projectifs . . . . . . . . . . . . 119

C.4.b Schémas en groupes sur un corps . . . . . . . . . . . . . . . 119

C.4.c Lissité des sous-groupes de $\mathbb{G}_{\mathrm{a}}^{g}$ et un calcul de $\Omega^{1}$. . . . . . . . 120

C.4.d Sous-groupes de $\mathbb{G}_{\mathrm{m}}^{g} \ldots \ldots \ldots \ldots$. . . . . . . . . 123

C.4.e Translations . . . . . . . . . . . . . . . 124

C.4.f Multiplication par $n \ldots \ldots \ldots \ldots . \ldots \ldots$

C.5 Dérivations et opérateurs différentiels . . . . . . . . . . . . . 125

C.5.a Dérivations ....................... 125

C.5.b Opérateurs différentiels . . . . . . . . . . . . 126 
Références Bibliographiques

vii 
Table des matières

viii 


\section{Notations et conventions générales}

$\circledast$ Tout au long de ce texte, nous désignerons par une lettre grasse $\mathbf{X}$ un multiplet de variables $\left(X_{1}, \ldots, X_{g}\right)$. Un multiplet de réels $\left(t_{1}, \ldots, t_{g}\right)$ sera noté, indifféremment, $\underline{t}$ ou $\mathbf{t}$. La notation $\mathrm{X}^{\mathrm{t}}$ désigne le produit $X_{1}^{t_{1}} \cdots X_{g}^{t_{g}}$, $|\underline{t}|$ est la somme $t_{1}+\cdots+t_{g}$ (nous parlerons aussi de la longueur de $\underline{t}$ ) et, si $\underline{t} \in \mathbb{N}^{g}$ alors $\underline{t} !:=t_{1} ! \cdots t_{g}$ !

$\circledast[x]$ désigne la partie entière du réel $x$.

$\circledast$ Si $x$ est un réel, le symbole $x^{+}$désigne le maximum de $x$ et 0 .

$\circledast \overline{\mathbb{Q}}$ désigne la clôture algébrique de $\mathbb{Q}$ dans $\mathbb{C}$ et $\overline{\mathbb{Z}}$ est l'ensemble des entiers algébriques dans $\overline{\mathbb{Q}}$.

$\circledast \mathbb{P}_{\mathbb{Z}}^{N}$ désigne l'espace projectif $\operatorname{Proj}\left(\mathbb{Z}\left[X_{0}, \ldots, X_{N}\right]\right)$.

$\circledast$ Soit $\mathbb{K}$ un corps de nombres. Nous désignons par $M_{\mathbb{K}}$ l'ensemble des places de $\mathbb{K}$. Soit $v \in M_{\mathbb{K}}$. La valeur absolue $|\cdot|_{v}$ sur le complété $\mathbb{K}_{v}$ est normalisée de la manière suivante :

$$
\left\{\begin{array}{l}
|1|_{v}=1 \quad \text { si } v \text { est archimédienne, } \\
|p|_{v}=\frac{1}{p} \quad \text { si } v \text { est ultramétrique, au-dessus du nombre premier } p .
\end{array}\right.
$$

$\circledast$ Si $\mathbf{x}=\left(x_{0}: \cdots: x_{g}\right) \in \mathbb{P}^{g}(\mathbb{K})\left(\mathbb{K}:\right.$ corps de nombres), le réel $h(\mathbf{x})$ (noté aussi $\left.h_{\text {Weil }}(\mathbf{x})\right)$ est la hauteur de Weil logarithmique absolue de $\mathbf{x}$ :

$$
h(\mathbf{x}):=\frac{1}{[\mathbb{K}: \mathbb{Q}]} \sum_{v \in M_{\mathbb{K}}}\left[\mathbb{K}_{v}: \mathbb{Q}_{v}\right] \log \max \left\{\left|x_{0}\right|_{v}, \ldots,\left|x_{g}\right|_{v}\right\}
$$

(cf. [84], p. 77). Lorsque $a_{1}, \ldots, a_{h} \in \overline{\mathbb{Q}}$ sont les coefficients d'un polynôme $P$, nous noterons $h(P)$ la hauteur du point projectif $\left(1: a_{1}: \cdots: a_{h}\right)$.

$\circledast$ Soient $g$ un entier strictement positif et $E$ un sous-espace vectoriel de $\overline{\mathbb{Q}}^{g}$, de codimension $\ell$. Fixons une base e de $\overline{\mathbb{Q}}^{g}$ et considérons un système d'équations, à coefficients algébriques, $\beta_{i, 1} z_{1}+\cdots+\beta_{i, g} z_{g}=0(1 \leq i \leq \ell)$ définissant $E$. Alors, la hauteur de $E$, notée $h(E)$, relative à la base e, est la somme des hauteurs des points $\left(\beta_{i, 1}: \cdots: \beta_{i, g}\right) \in \mathbb{P}^{g-1}(\overline{\mathbb{Q}})$. Cette définition est relativement arbitraire et de pure convenance (sauf lorsque $E$ est un hyperplan). À une fonction de $g$ près, cette notion de hauteur rejoint celle définie par W.M. ScHMIDT [71], ainsi que celle, plus arakelovienne, définie par J.-B. Bost dans [19], page 133.

$\circledast$ Soient $N, a$ des entiers non nuls et $\mathbb{K}$ un corps. Nous désignons par $\mathbb{K}\left[\mathbb{P}^{N}\right]_{a}$ l'ensemble des polynômes homogènes de degré $a$ en les variables $X_{0}, \ldots, X_{N}$, à coefficients dans $\mathbb{K}$, et $\mathbb{K}\left[\mathbb{P}^{N}\right]$ l'union des $\mathbb{K}\left[\mathbb{P}^{N}\right]_{a}, a \in \mathbb{N}$. Autrement dit, $\mathbb{K}\left[\mathbb{P}^{N}\right]$ est l'ensemble des polynômes homogènes à $N+1$ variables et à coefficients dans $\mathbb{K}$. 
$\circledast \mathrm{Si} \mathbb{K}$ est un corps (commutatif) et si $\mathbf{v}=\left(v_{1}, \ldots, v_{n}\right)$ est un élément de $\mathbb{K}^{n}$ alors

$$
\mathcal{D}_{\mathbf{v}}:=v_{1} \frac{\partial}{\partial z_{1}}+\cdots+v_{n} \frac{\partial}{\partial z_{n}} .
$$

Considérons $\underline{t} \in \mathbb{N}^{g}$ et $\mathbf{e}=\left\{\mathbf{e}_{1}, \ldots, \mathbf{e}_{g}\right\}$ une famille d'éléments de $\mathbb{K}^{n}$, alors

$$
\mathcal{D} \frac{t}{\mathbf{e}}:=\mathcal{D}_{\mathbf{e}_{1}}^{t_{1}} \cdots \mathcal{D}_{\mathbf{e}_{g}}^{t_{g}} .
$$

$\circledast$ Dans ce texte, $\ln =\log$ est le logarithme népérien (éventuellement étendu à un ouvert simplement connexe de $\mathbb{C} \backslash\{0\})$. Et $\log ^{+}$désigne la fonction $x \mapsto \log \max \{1, x\}$.

$\circledast$ Si $\mathbf{G}$ est un groupe algébrique sur un corps $\mathbb{K}$, la notation $t_{\mathbf{G}}$ désigne le $\mathbb{K}$-espace tangent à l'origine de $\mathbf{G}$. Si $\mathbb{K}=\mathbb{C}, \Omega_{\mathbf{G}(\mathbb{C})}$ désigne le sous-groupe des périodes de $\mathbf{G}$.

$\circledast$ Si $\mathbf{G}$ est un groupe algébrique défini sur un corps $\mathbb{K}$ et $\mathbb{M}$ est une extension de $\mathbb{K}$, alors $\mathbf{G}_{\mathbb{M}}$ désigne le groupe $\mathbf{G} \times_{\text {Spec } \mathbb{K}} \operatorname{Spec} \mathbb{M}$.

$\circledast$ Au cours de la démonstration des théorèmes du $\S$ I.3.a apparaissent des constantes $c$, indexées par $\mathbb{N}$, qui ne dépendent que du groupe algébrique $\mathbf{G}$ et d'un éventuel plongement de ce groupe dans un espace projectif. Par convention, ces constantes seront positives et choisies de manière croissante en fonction de leur indice $\left(c_{1} \leq c_{2} \leq \cdots\right)$.

$\circledast$ Lorsque $n$ est un entier naturel et $\mathbf{p}$ un point complexe d'un groupe algébrique, $\Gamma_{\mathbf{p}}(n)$ désignera l'ensemble $\{0, \mathbf{p}, \ldots, n \mathbf{p}\}$.

$\circledast$ card désigne le cardinal d'un ensemble et Vol le volume d'un objet.

$\circledast$ Si $\alpha$ est un nombre algébrique (sur $\mathbb{Q}$ ), $|\bar{\alpha}|$ désigne le maximum des valeurs absolues des conjugués de $\alpha$.

$\circledast$ Si $\mathbb{K}$ est un corps de nombres, nous désignerons par $\mathcal{O}_{\mathbb{K}}$ l'anneau des entiers de $\mathbb{K}$.

$\circledast$ Si $V=V_{1} \times \cdots \times V_{n}$ est une variété sur un corps $\mathbb{K}, \mathscr{H}\left(V ; X_{1}, \ldots, X_{n}\right)$ désigne la partie homogène de plus haut degré du polynôme de Hilbert-Samuel multihomogène de $V$, multipliée $\operatorname{par}(\operatorname{dim} V)$ ! (voir $\S 5.2 .3$ de [84]). 


\title{
Introduction
}

\author{
ProblémATique
}

Cette thèse s'inscrit dans la lignée des nombreux travaux relatifs à la théorie des formes linéaires de logarithmes.

Étant donné des nombres algébriques $\alpha_{1}, \ldots, \alpha_{n}$ non nuls et le choix de logarithmes $\lambda_{i}$ de $\alpha_{i}$ (i.e. $\left.\alpha_{i}=e^{\lambda_{i}}\right)$, A. BAKER a démontré que la famille $\left\{1, \lambda_{1}, \ldots, \lambda_{n}\right\}$ est libre sur le corps des nombres algébriques $\overline{\mathbb{Q}}$ si la famille $\left\{\lambda_{1}, \ldots, \lambda_{n}\right\}$ l'est sur $\mathbb{Q}[2]$. Ce théorème est apparu comme la conséquence d'un résultat plus général. Considérons $\beta_{0}, \ldots, \beta_{n}$ des nombres algébriques non tous nuls. Sous l'hypothèse de linéaire indépendance sur $\mathbb{Q}$ des $\lambda_{i}$, Baker fournit (ibid.) une minoration de la quantité

$$
\log \left|\beta_{0}+\beta_{1} \lambda_{1}+\cdots+\beta_{n} \lambda_{n}\right|
$$

polynomiale en la hauteur des $\beta_{i}$. Ces résultats marquent le début de l'histoire des mesures d'indépendance linéaire de logarithmes.

Nous savons que ces questions s'inscrivent naturellement dans le cadre des groupes algébriques commutatifs, définis sur $\overline{\mathbb{Q}}[49,50,80]$. Étant donné un tel groupe $\mathbf{G}$, d'espace tangent à l'origine $t_{\mathbf{G}}$, un logarithme $\mathbf{u} \in t_{\mathbf{G}}(\mathbb{C})$ d'exponentielle algébrique (i.e. $\exp (\mathbf{u}) \in \mathbf{G}(\overline{\mathbb{Q}})$ où $\exp$ est l'exponentielle du groupe de Lie complexe $\mathbf{G}(\mathbb{C})$ ) et un hyperplan $W$ de $t_{\mathbf{G}}$, que peut-on dire de la distance de $\mathbf{u}$ à $W$ ? Les travaux de Baker étudient le cas des groupes linéaires (commutatifs). Cette problématique se scinde immédiatement en deux parties :

(1) - expliquer les raisons pour lesquelles $\mathbf{u}$ peut appartenir à $W$,

(2) - minorer la distance $\mathrm{d}(\mathbf{u}, W)$ (si elle n'est pas nulle).

Dans ce cadre général, G. WÜsThOLZ [85] a apporté un élément de réponse à la première question en démontrant que si $\mathbf{u}$ appartient à $W$ alors il existe un sous-groupe algébrique $\widetilde{\mathbf{G}}$ de $\mathbf{G}$, d'espace tangent inclus dans $W$, tel que $\mathbf{u} \in t_{\widetilde{\mathbf{G}}}(\mathbb{C})$. Ce résultat généralise le théorème de Baker (énoncé qualitatif). Le problème devient :

(1)' - Si $\mathbf{u} \in W$, quelles informations apporter sur un sous-groupe (algébrique) $\widetilde{\mathbf{G}}$ tel que $\mathbf{u} \in t_{\widetilde{\mathbf{G}}}(\mathbb{C})$ ? Peut-on, par exemple, estimer le covolume de son groupe des périodes?

Les paramètres qui entrent en jeu dans ces questions ont trois origines distinctes:

$\boldsymbol{X}$ Le groupe $\mathbf{G}$ : sa dimension, le degré d'un corps (de nombres) de définition, les caractéristiques d'un plongement de $\mathbf{G}$ dans un espace projectif*, une distance sur $t_{\mathbf{G}}(\mathbb{C})$;

*Si G est une variété abélienne, c'est le choix d'une polarisation. 


\section{Introduction}

$\boldsymbol{X}$ Le logarithme $\mathbf{u}$ : sa norme, sa hauteur et le degré d'un corps de définition de $\exp (\mathbf{u})$; $\boldsymbol{x}$ L'hyperplan $W$ : sa hauteur et le degré d'un corps de définition.

La question (1)' (ou une de ses variantes) a été l'objet de plusieurs articles $[3,7,9,10,55]$ dans le cas d'un groupe linéaire commutatif, et a presque toujours été éludée dans les autres cas. En fait, bien que la méthode de démonstration au cœur des articles [45, 65] fournisse une majoration du covolume du sous-groupe des périodes de $\widetilde{\mathbf{G}}$ (et même, un peu mieux, du degré de $\widetilde{\mathbf{G}}$ ), très peu de résultats ont été publiés. Nous citerons S. DAVID [30] dans le cas où le groupe $\mathbf{G}$ est le produit du groupe $\mathbb{G}_{\mathrm{a}}$ et de courbes elliptiques, D. BERTRAND [6] qui s'est intéressé à une variante de la question (1)' lorsque $\mathbf{G}$ est une variété abélienne et V. Bosser [15] dans le contexte légèrement différent des modules de Drinfeld.

Quant au second aspect du problème (question (2)), le cas d'un groupe linéaire a sans aucun doute été le plus étudié en raison de son aspect élémentaire et de ses multiples applications en arithmétique (voir [35], § 2 du chap. 4). Pour un groupe algébrique commutatif quelconque, la première réponse a été donnée par P. PHILIPPON et M. WALDSCHMidT [65] et suivie, quelques années plus tard, d'une importante amélioration par N. HIRATA-KoHNo [45].

En dehors de ces résultats généraux, beaucoup d'articles traitent de cas particuliers (logarithmes d'un produit de courbes elliptiques CM ; G tore et $W$ hyperplan défini sur $\mathbb{Q}$, etc.) avec des preuves et des outils très variés (polynômes binomiaux ou de Lagrange ; déterminants d'interpolation, etc.).

Les réponses apportées aux questions (1)' et (2) varient en fonction, d'une part, des paramètres mis en valeur (en vue, par exemple, de résoudre certaines équations diophantiennes *) et, d'autre part, de la méthode de démonstration choisie (et des outils qu'elle autorise).

\section{COntenu DE LA THĖSE}

Elle comporte deux parties indépendantes ainsi que trois annexes.

La première partie traite le cas général d'un groupe algébrique commutatif, défini sur $\overline{\mathbb{Q}}$. Nous apportons de nouvelles réponses aux questions (1)' et (2) qui améliorent les résultats connus auparavant (pour les groupes algébriques commutatifs quelconques), en généralisant les techniques de [31]. En particulier, nous obtenons une minoration de la distance de $\mathbf{u}$ à $W$ optimale en la hauteur de l'hyperplan $W$ et plus précise que la mesure d'indépendance linéaire de N. Hirata-Kohno [45] en la hauteur du point $\exp (\mathbf{u})$. Pour obtenir ces avancées, nous avons modifié la partie arithmétique de la démonstration. Nous utilisons le procédé de changement de variable de CHUDNOvSKY, dans le cadre des groupes formels, pour majorer plus précisément qu'auparavant les normes ultramétriques de nombres algébriques construits au cours de la preuve.

La seconde partie adopte un point de vue totalement différent, bien que le lemme clef de la démonstration précédente soit utilisé de nouveau. Nous étudions le cas particulier du

\footnotetext{
*Ces considérations expliquent pourquoi la mesure donnée dans [52], bien qu'assez mauvaise d'un point de vue théorique, mais avec une constante numérique très faible, est si utile pour la résolution pratique d'équations diophantiennes $[11,26,53,54,57,58,62]$.
} 
groupe produit (direct) d'une variété abélienne (principalement polarisée) par le groupe additif $\mathbb{G}_{a}$, avec une hypothèse (technique) supplémentaire sur le logarithme u (« cas abélien nonhomogène »). Dans ce cadre restreint, nous établissons une nouvelle mesure d'indépendance linéaire comparable à celles données dans la première partie mais totalement explicite en les invariants liés à la variété abélienne (dimension, hauteur de Faltings). C'est le point de vue adopté dans [30] dans le cas où la variété abélienne est un produit de courbes elliptiques, avec, cependant, ici, une méthode et des outils très différents. La particularité de cette seconde partie est surtout de mettre en œuvre, pour la première fois dans ce contexte, la méthode des pentes de J.-B. Bost [19] et certains résultats de Géométrie d'Arakelov qui lui sont naturellement attachés. 
Introduction

xiv 


\section{Mesure d'indépendance linéaire de logarithmes I}





\section{Le cas général}

\section{$\S 1$. Introduction}

Étant donné un groupe algébrique commutatif $\mathbf{G}$ défini sur $\overline{\mathbb{Q}}$, un hyperplan $W$ de l'espace tangent à l'origine de $\mathbf{G}$ et $\mathbf{u}$ un point complexe de cet espace tangent, d'exponentielle (de Lie) algébrique, nous étudions la distance qui sépare $\mathbf{u}$ de cet hyperplan $W$.

Si $\mathbf{u} \in W$, un théorème de Wüstholz [86] assure l'existence d'un sous-groupe $\widetilde{\mathbf{G}}$ de $\mathbf{G}$ tel que $\mathbf{u} \in t_{\widetilde{\mathbf{G}}}(\mathbb{C}) \subseteq W \otimes \mathbb{C}$. Nous redémontrons ce théorème en apportant une précision supplémentaire sur le groupe $\widetilde{\mathbf{G}}$ : son degré (relatif à un plongement fixé de $\mathbf{G}$ dans un espace projectif) admet un majorant effectif, indépendant de la hauteur de l'espace vectoriel $W$. Cette précision permet de quantifier la relation de dépendance algébrique entre les coordonnées de $\mathbf{u}$. Par exemple, dans le cas particulier où $\mathbf{G}$ est une variété semi-abélienne* et $W$ un hyperplan, une conséquence du théorème que nous obtenons est le résultat suivant :

Théorème I.1.1. Soit $\mathbf{G}$ une variété semi-abélienne, définie sur $\overline{\mathbb{Q}}$ et munie d'un plongement $\Phi$ dans un espace projectif. Soient $W$ un hyperplan de l'espace tangent $t_{\mathbf{G}}$ et $\mathbf{u} \in W \otimes \mathbb{C}$ tel que $\mathbf{p}:=\exp _{\mathbf{G}(\mathbb{C})} \mathbf{u} \in \mathbf{G}(\overline{\mathbb{Q}})$. Soient $h(\mathbf{p})$ la hauteur du point (projectif) $\Phi(\mathbf{p})$ et $\|$.$\| une norme$ hermitienne sur $t_{\mathbf{G}} \otimes \mathbb{C}$.

Alors il existe une constante $c_{1}=c_{1}(\mathbf{G}, \Phi,\|\mathbf{u}\|,[\mathbb{Q}(\mathbf{p}): \mathbb{Q}]$ ) (indépendante de la hauteur de $\mathbf{p}$ et de la hauteur de $W$ ) et un sous-groupe algébrique connexe $\widetilde{\mathbf{G}}$ de $\mathbf{G}$, de dimension $\widetilde{d}$, vérifiant les conditions suivantes :

(1) $\mathbf{u} \in t_{\widetilde{\mathbf{G}}}(\mathbb{C}) \subseteq W \otimes \mathbb{C}$

(2) $\operatorname{deg}_{\Phi} \widetilde{\mathbf{G}} \leq c_{1}\left(\frac{\max \{1, h(\mathbf{p})\}}{\log \max \{e, h(\mathbf{p})\}}\right)^{\widetilde{d}}$

Ce type d'énoncé est rarement mis en relief en dépit de la (relative) simplicité avec laquelle il se déduit de la démonstration d'une mesure d'indépendance linéaire de logarithmes. Dans [30], p. 10, S. DAVID fournit une majoration du degré de $\widetilde{\mathbf{G}}$, dans le cas particulier où $\mathbf{G}$ est un produit de courbes elliptiques, avec une constante $c_{1}$ explicite (mais sa borne dépend de la hauteur de $W$ ). L'énoncé du théorème I.1.1 est assez simple mais il n'est pas assez précis pour les applications éventuelles. Aussi, à l'intention du lecteur « spécial », nous en donnons ci-après une version plus explicite.

Théorème I.1.1 bis. Soit $\mathbf{G}$ une variété semi-abélienne définie sur $\overline{\mathbb{Q}}$, munie d'un plongement $\Phi$ dans un espace projectif. Soient $W$ un hyperplan de l'espace tangent $t_{\mathbf{G}}$ et $\mathbf{u} \in W \otimes \mathbb{C}$ tel que $\mathbf{p}:=\exp _{\mathbf{G}(\mathbb{C})} \mathbf{u} \in \mathbf{G}(\overline{\mathbb{Q}})$.

${ }^{*}$ Un groupe algébrique $\mathfrak{G}$, sur un corps de caractéristique nulle, est dit semi-abélien s'il existe un tore $\mathfrak{L}$, une variété abélienne $\mathfrak{A}$ et une suite exacte (de groupes algébriques) $0 \rightarrow \mathfrak{L} \rightarrow \mathfrak{G} \rightarrow \mathfrak{A} \rightarrow 0$. 
Soient $h(\mathbf{p})$ la hauteur du point (projectif) $\Phi(\mathbf{p})$ et $\|\cdot\|$ une norme hermitienne sur $t_{\mathbf{G}} \otimes \mathbb{C}$. Soit D un majorant du degré d'un corps de définition de $\mathbf{G}, W, \mathbf{p}$. Enfin définissons le réel $E \geq$ e par

$$
E^{2}=e^{2} \frac{D}{\|\mathbf{u}\|^{2}} \max \left\{1, h(\mathbf{p}), \frac{\|\mathbf{u}\|^{2}}{D}\right\} .
$$

Alors il existe une constante $c_{2}=c_{2}(\mathbf{G}, \Phi,\|$.$\| ) (en particulier indépendante de la hauteur de \mathbf{p}$ et de la hauteur de $W$ ) et il existe un sous-groupe algébrique connexe $\widetilde{\mathbf{G}}$ de $\mathbf{G}$, de dimension $\widetilde{d}$, vérifiant les conditions suivantes :

$$
\begin{aligned}
& \text { (1) } \mathbf{u} \in t_{\widetilde{\mathbf{G}}}(\mathbb{C}) \subseteq W \otimes \mathbb{C} \\
& \qquad \operatorname{deg}_{\Phi} \widetilde{\mathbf{G}} \leq c_{1} \max \left\{1, \frac{D \log (E\|\mathbf{u}\|)}{\log E}\right\} \times \max \left\{1, \frac{(E\|\mathbf{u}\|)^{2}}{\log E}, \frac{D(E\|\mathbf{u}\|)^{2} \log (E\|\mathbf{u}\|)}{(\log E)^{2}}\right\}^{\widetilde{d}} .
\end{aligned}
$$

Lorsque $\mathbf{u} \notin W$, nous obtenons une minoration de la distance $\mathrm{d}(\mathbf{u}, W)$, optimale en la hauteur $h(W)$ de l'espace $W$ :

Théorème I.1.2. Soit $\mathbf{G}$ un groupe algébrique commutatif, défini sur $\overline{\mathbb{Q}}$. Soit $W$ un hyperplan de l'espace tangent à l'origine $t_{\mathbf{G}}$, défini sur un corps de nombres de degré $D$, et soit $\mathbf{u} \in$ $t_{\mathbf{G}}(\mathbb{C})$, d'exponentielle algébrique. Munissons $t_{\mathbf{G}}(\mathbb{C})$ d'une distance hermitienne $\mathrm{d}$. Il existe une constante $c_{3}=c_{3}(\mathbf{G}, \mathrm{d}, \mathbf{u}, D)$ (indépendante de la hauteur de $W$ ) telle que, si $\mathbf{u} \notin W$, alors

$$
\log \mathrm{d}(\mathbf{u}, W) \geq-c_{3} \max \{1, h(W)\} .
$$

Ce théorème améliore la dépendance en $h(W)$ du résultat antérieur de N. HiRATA-KoHNo [46], qui comportait une puissance de $\log h(W)$ supplémentaire. Il est optimal. De ce point de vue, nous généralisons également les travaux récents de M. ABLy [1], S. DAVID et N. HiRATAKoHNo [31], qui contenaient des hypothèses restrictives sur le groupe G. Pour un historique détaillé des progrès réalisés sur cette question, nous renvoyons le lecteur à [31].

Dans le cadre général d'un groupe algébrique commutatif, toutes les mesures d'indépendance linéaire de logarithmes, obtenues jusqu'à maintenant, ont leur schéma de démonstration fondé sur l'article original de P. PHILIPPON et M. WALDSCHMIDT [65], avec en toile de fond la méthode de Baker. En 1991, N. HiratA-KoHNo introduisit une nouvelle idée qui permit d'améliorer considérablement la dépendance de la mesure en le paramètre « hauteur de l'hyperplan ». Cette idée consiste à modifier légèrement le groupe algébrique et l'hyperplan de départ, puis à se servir d'un facteur $\mathbb{G}_{a}$ pour juguler l'influence des dérivations. Le nouvel argument qui optimise la mesure pour ce paramètre (théorème I.1.2 ci-dessus) est dû à G.V. Chudnovsky [27, 28, 29], mais a été mis en œuvre pour la première fois dans ce contexte par S. David et N. Hirata-Kohno [31]. Il repose sur un (simple !) changement de variable qui donne lieu à un raisonnement direct avec les paramètres (locaux) du groupe algébrique et non, comme auparavant, avec ceux de l'espace tangent du groupe. Cela revient à utiliser le logarithme (formel) en lieu et place de l'exponentielle (ce qui, au fond, pour obtenir des formes linéaires de logarithmes peut sembler être une idée bien naturelle) pour les estimations des normes ultramétriques des nombres algébriques qui apparaissent au cours de notre démonstration. C'est donc la méthode générale de [65], précédée d'un conditionnement des données (groupe algébrique, hyperplan) et à laquelle s'ajoute le choix pertinent des variables locales, qui permet d'obtenir les résultats du paragraphe I.3.a et d'en déduire en particulier les théorèmes I.1.1 et I.1.2. 
Signalons qu'il est possible de donner un aspect beaucoup plus intrinsèque et géométrique aux résultats et à leurs démonstrations en utilisant la méthode des pentes de J.-B. Bost (c'est l'objet de la seconde partie de cette thèse). C'est cette méthode qui est probablement la plus efficace pour calculer la constante qui apparaît dans le théorème I.3.2. Elle fournit un cadre naturel aux démonstrations de transcendance. À titre d'exemple, lorsque $\mathbf{G}$ est une variété abélienne, J.-B. Bost a obtenu une majoration totalement explicite du degré de $\widetilde{\mathbf{G}}$ dans le théorème I.1.1 ci-dessus, avec, cependant, une dépendance (polynomiale) en $h(W)$ ( $c f$. proposition 5.2 de [19]). Malheureusement, cette méthode ne possède pas, en l'état actuel, la souplesse requise pour s'adapter directement au cadre de notre étude (la méthode des déterminants d'interpolation de M. LAURENT non plus, du reste). En particulier, les formes de raisonnement assez « tortueuses » qu'autorise le fameux « polynôme auxiliaire » de la transcendance sont parfois plus difficiles (un des difficultés est expliquée en détail dans la seconde partie) à mettre en œuvre avec ces méthodes. Concrètement, la méthode des pentes sépare nettement les places ultramétriques des places archimédiennes (on évalue les normes d'un morphisme d'évaluation séparément pour ces deux types de places et de manière indépendante). Avec la méthode du polynôme auxiliaire, on extrapole préalablement pour majorer (finement) les dérivées en une place archimédienne puis, c'est la première dérivée non-nulle que l'on évalue aux places ultramétriques. L'ordre dans lequel on effectue les opérations est crucial : il serait impossible (dans ce que nous appellerons le cas périodique) d'évaluer précisément les dérivées de la fonction auxiliaire aux places ultramétriques sans avoir extrapolé auparavant.

\section{$\S$ 2. Données}

\section{$\S$ 2.a. Le groupe algébrique}

Soient $n$ un entier naturel non nul et $\mathbf{K}$ un corps de nombres plongé dans $\mathbb{C}$ (nous noterons parfois $\sigma_{0}: \mathbf{K} \rightarrow \mathbb{C}$ le plongement choisi de $\mathbf{K}$ dans $\mathbb{C}$ ). Considérons $\mathbf{G}_{1}, \ldots, \mathbf{G}_{n}$ des groupes algébriques commutatifs, définis sur K. Notons

$$
\mathbf{G}_{0}:=\mathbb{G}_{\mathrm{a}}, \quad \mathbf{G}:=\mathbf{G}_{0} \times \mathbf{G}_{1} \times \cdots \times \mathbf{G}_{n}
$$

le groupe algébrique produit, $\delta_{\ell}$ la dimension de $\mathbf{G}_{\ell}$ et $d+1:=\delta_{0}+\delta_{1}+\ldots+\delta_{n}$ celle de $\mathbf{G}$. Soit $\mathbf{e}:=\left(e_{m}\right)_{0 \leq m \leq d}$ une base de l'espace tangent $t_{\mathbf{G}}$ telle que

$$
\mathbf{e}_{i}=\left(e_{\delta_{0}+\cdots+\delta_{i-1}}, \ldots, e_{\delta_{0}+\cdots+\delta_{i}-1}\right)
$$

forme une base de $t_{\mathbf{G}_{i}}$ pour $i \in\{0, \ldots, n\}$ (par convention d'écriture, $\delta_{-1}:=-1$ ). Enfin, nous choisissons un plongement admissible* $\Phi$ du groupe $\mathbf{G}$ dans un espace multi-projectif $\mathbb{P}:=\mathbb{P}^{N_{0}} \times \cdots \times \mathbb{P}^{N_{n}}$, et nous noterons $\rho_{j}$ un majorant $\geq 1$ de l'ordre ${ }^{\dagger}$ d'une représentation de

*Au sens du $§$ I.5. On dit aussi parfois plongement « à la Serre », en référence à l'appendice [73] de [80], écrit par J.-P. SERRE sur ce sujet.

${ }^{\dagger}$ Lorsque $f\left(z_{1}, \ldots, z_{\ell}\right)$ est une fonction analytique à $\ell$ variables, l'ordre de $f$ est par définition

$$
\limsup _{R \rightarrow \infty} \frac{\log \log ^{+} \max _{\left|z_{i}\right| \leq R}\left|f\left(z_{1}, \ldots, z_{\ell}\right)\right|}{\log R} .
$$


l'exponentielle du groupe de Lie $\mathbf{G}_{j}(\mathbb{C})$ dans ce plongement. Dans la pratique, nous prendrons $\rho_{j}=1$ si $\mathbf{G}_{j}$ est un groupe linéaire (commutatif) et $\rho_{j}=2$ si $\mathbf{G}_{j}$ a une partie abélienne non triviale.

\section{§ 2.b. Données arithmétiques}

Soient $\mathbf{L}$ un corps de nombres contenant $\mathbf{K}, D$ l'entier naturel $[\mathbf{L}: \mathbb{Q}]$ et $\mathbf{p} \in \mathbf{G}(\mathbf{L})$. Soit $\xi_{1} \ldots, \xi_{D}$ une base quelconque du $\mathbb{Q}$-espace vectoriel L. Conformément au paragraphe « Notations et conventions générales », le réel $h\left(\xi_{1}: \cdots: \xi_{D}\right)$ est la hauteur logarithmique absolue de $\left(\xi_{1}: \cdots: \xi_{D}\right) \in \mathbb{P}^{D-1}(\mathbf{L})$. Nous supposerons que $\mathbf{L}$ est plongé dans $\mathbb{C}$ via un prolongement de $\sigma_{0}$ (que nous noterons encore $\sigma_{0}$ ).

Considérons $\mathbf{u}=u_{0} e_{0}+\cdots+u_{d} e_{d} \in t_{\mathbf{G}}(\mathbb{C})$ un logarithme de $\mathbf{p}$, i.e.

$$
\exp _{\mathbf{G}(\mathbb{C})}(\mathbf{u})=\mathbf{p}
$$

(en particulier $u_{0} \in \mathbf{L}$ ). Nous notons $\mathbf{p}_{j}$ l'image de $\mathbf{p}$ dans $\mathbf{G}_{j}$ et $\mathbf{u}_{j}$ un logarithme de $\mathbf{p}_{j}$ tel que

$$
\sum_{j=0}^{n} \mathbf{u}_{j}=\mathbf{u}
$$

Le réel $h\left(\mathbf{p}_{j}\right)$ est la hauteur (logarithmique absolue) du point $\Phi\left(\mathbf{p}_{j}\right)$ dans l'espace projectif $\mathbb{P}^{N_{j}}$.

Enfin soit $W$ un hyperplan de $t_{\mathbf{G}} \otimes_{\mathbf{K}} \mathbf{L}$ d'équation

$$
z_{0}=\beta_{1} z_{1}+\cdots+\beta_{d} z_{d}
$$

dans la base e, où $\beta_{1}, \ldots, \beta_{d}$ sont des éléments de $\mathbf{L}$. Rappelons que, pour nous, la hauteur $h(W)$ de $W$ est la hauteur logarithmique absolue de $\left(1: \beta_{1}: \cdots: \beta_{d}\right)$.

Posons également

$$
\Lambda:=u_{0}-\beta_{1} u_{1}-\cdots-\beta_{d} u_{d} .
$$

Le théorème porte sur une minoration de la distance de $\mathbf{u}$ à $W$ lorsque cette quantité n'est pas nulle. Pour énoncer le théorème du paragraphe suivant, nous fixons une norme hermitienne $\|$. $\|$ sur $t_{\mathbf{G}}(\mathbb{C})$ (et notons d la distance associée à cette norme). Par exemple, si nous choisissons la norme qui rend la base e orthonormée, nous avons

$$
\mathrm{d}(\mathbf{u}, W)=\frac{|\Lambda|}{\left(1+\left|\beta_{1}\right|^{2}+\cdots+\left|\beta_{d}\right|^{2}\right)^{1 / 2}} .
$$

Ainsi, il est équivalent de minorer $\mathrm{d}(\mathbf{u}, W)$ et de minorer le module d'une forme linéaire de logarithmes.

\section{§ 3. Résultats}

\section{$\S$ 3.a. Énoncés}

Théorème I.3.1. Il existe une constante (effective) $c_{4}>0$, ne dépendant que du triplet $(\mathbf{G}, \Phi, \mathrm{d})$, ayant la propriété suivante. Soit $E$ un réel $\geq$ e. Posons

$$
\mathfrak{a}:=1+\left[\frac{D \log \left(D+\sum_{j=1}^{n}\left(h\left(\mathbf{p}_{j}\right)+E\left\|\mathbf{u}_{j}\right\|\right)\right)}{\log E}\right]
$$


et

$$
\begin{aligned}
U:= & \frac{\operatorname{card}\left(\Gamma_{\mathbf{p}}(\mathfrak{a})\right)}{(\mathfrak{a} \log E)^{d}} \times\left(D h(W)+D h\left(u_{0}\right)+\mathfrak{a} \cdot \log E\right) \\
& \times \prod_{j=1}^{n}\left(\mathfrak{a} \log E+D \max _{0 \leq s \leq c_{4} \mathfrak{a}}\left\{h\left(s \mathbf{p}_{j}\right)\right\}+\left(E \mathfrak{a}\left\|\mathbf{u}_{j}\right\|\right)^{\rho_{j}}\right)^{\delta_{j}} .
\end{aligned}
$$

- Si $\mathbf{u} \in W_{\mathbb{C}}$, alors il existe un sous-groupe algébrique connexe $\widetilde{\mathbf{G}}$ de $\mathbf{G}_{\mathbf{L}}$, de dimension $\widetilde{d}$, tel que

(a) L'espace tangent à l'origine $t_{\widetilde{\mathbf{G}}}$ est inclus dans l'hyperplan $W$.

(b) Le point $\mathbf{u}$ appartient $\grave{a} t_{\widetilde{\mathbf{G}}}(\mathbb{C})$.

(c) Si $\pi: \mathbf{G} \longrightarrow \prod_{j=1}^{n} \mathbf{G}_{j}$ désigne la projection canonique, il existe un entier $m \geq 1$ et des entiers distincts $1 \leq j_{1}<\cdots<j_{m} \leq n$ tels que le degré de $\pi(\widetilde{\mathbf{G}})$ (relatif au plongement choisi de $\mathbf{G}$ dans un espace projectif) vérifie

$$
\begin{aligned}
\operatorname{deg}_{\Phi} \pi(\widetilde{\mathbf{G}}) \leq & c_{4} \frac{\operatorname{card}\left(\Gamma_{\mathbf{p}}(\mathfrak{a})\right)}{(\mathfrak{a} \log E)^{\tilde{d}}} \times \prod_{\ell=1}^{m-1}\left(\mathfrak{a} \log E+D \max _{0 \leq s \leq c_{4} \mathfrak{a}}\left\{h\left(s \mathbf{p}_{j_{\ell}}\right)\right\}+\left(E \mathfrak{a}\left\|\mathbf{u}_{j_{\ell}}\right\|\right)^{\rho_{j_{\ell}}}\right)^{\delta_{j_{\ell}}} \\
& \times\left(\mathfrak{a} \log E+D \max _{0 \leq s \leq c_{4} \mathfrak{a}}\left\{h\left(s \mathbf{p}_{j_{m}}\right)\right\}+\left(E \mathfrak{a}\left\|\mathbf{u}_{j_{m}}\right\|\right)^{\rho_{j_{m}}}\right)^{\widetilde{d}-\left(\delta_{j_{1}}+\cdots+\delta_{j_{m-1}}\right)} \\
& \times \max \left\{1, \frac{D h\left(\xi_{1}: \cdots: \xi_{D}\right)}{U}\right\} .
\end{aligned}
$$

- Si $\mathbf{u} \notin W_{\mathbb{C}}$, alors :

$$
\log \mathrm{d}(\mathbf{u}, W) \geq-c_{4} \max \left\{U, D h\left(\xi_{1}: \cdots: \xi_{D}\right)\right\}
$$

Dans le cas particulier où la forme linéaire est non-homogène, nous avons le résultat suivant plus précis.

Théorème I.3.2. Avec les notations du théorème I.3.1, supposons que le nombre complexe $u_{0}$ soit non nul et que le groupe algébrique $\prod_{j=1}^{n} \mathbf{G}_{j}$ soit une variété semi-abélienne. Alors $\mathbf{u}$ n'appartient pas à $W$ et il existe une constante $c_{5}>0$, ne dépendant que du triplet $(\mathbf{G}, \Phi, \mathrm{d})$, telle que, si

$$
\begin{aligned}
U^{\prime}:= & \frac{\mathfrak{a}}{(\mathfrak{a} \log E)^{d}} \times\left(D h(W)+D \max _{0 \leq s \leq c_{5} \mathfrak{a}}\left\{h\left(s u_{0}\right)\right\}+\log E\right) \\
& \times \prod_{j=1}^{n}\left(\mathfrak{a} \log E+D \max _{0 \leq s \leq c_{5} \mathfrak{a}}\left\{h\left(s \mathbf{p}_{j}\right)\right\}+\left(E \mathfrak{a}\left\|\mathbf{u}_{j}\right\|\right)^{\rho_{j}}\right)^{\delta_{j}}
\end{aligned}
$$

alors

$$
\log \mathrm{d}(\mathbf{u}, W) \geq-c_{5} \max \left\{U^{\prime}, D h\left(\xi_{1}: \cdots: \xi_{D}\right)\right\}
$$




\section{$\S 3 . b$. Commentaires et conséquences}

Ce théorème contient les mesures d'indépendance linéaires de logarithmes obtenues par N. Hirata-Kohno [45] et S. David [30], à l'exception, dans ce dernier cas, de la constante $c_{5}$ qui est totalement explicite. L'amélioration essentielle qu'apporte le théorème I.3.1 par rapport à ces résultats est la disparition de termes parasites en $\log h(W)$, pour garder un minorant de $\log \mathrm{d}(\mathbf{u}, W)$ linéaire en $h(W)$ (la quantité $D h\left(\xi_{1}: \cdots \xi_{D}\right)$ pouvant être majorée par une fonction linéaire de $h(W)$, voir ci-dessous). La majoration $\log |\Lambda| \geq-c_{6} \max \{1, h(W)\}$ est alors optimale*. Le majorant (2) de $\operatorname{deg} \pi(\widetilde{\mathbf{G}})$ est, quant à lui - et c'est nouveau - indépendant de $h(W)$.

Avec des arguments ad hoc, il est possible de démontrer des majorations plus précises du degré de $\pi(\widetilde{\mathbf{G}})$ lorsque $\mathbf{G}$ est un groupe linéaire commutatif (voir $\S$ I.3.c ci-après). Cependant, dans le cadre général d'un groupe algébrique commutatif quelconque, seule la méthode de Baker (que nous employons) permet, à l'heure actuelle, d'obtenir de tels renseignements. Par ailleurs, si nous nous sommes contentés de majorer le degré total de $\pi(\widetilde{\mathbf{G}})$, une adaptation triviale de la proposition I.9.3 permet de majorer individuellement chaque coefficient de degré maximal du polynôme de Hilbert-Samuel multihomogène de $\pi(\widetilde{\mathbf{G}})$ (i.e. le degré des projections de $\pi(\widetilde{\mathbf{G}})$ sur ses $« n$-faces d'indice $\left(i_{1}, \ldots, i_{n}\right) »$ de $\left.\mathbf{G}_{1} \times \cdots \times \mathbf{G}_{n}\right)$, à l'instar du théorème 2 de [7].

Il existe une constante $c_{7}$, ne dépendant que de $(\mathbf{G}, \Phi)$, telle que le terme $\max _{0 \leq s \leq \mathfrak{a}}\left\{h\left(s \mathbf{p}_{j}\right)\right\}$ soit majoré par $c_{7} \mathfrak{a}^{\rho_{j}}\left(h\left(\mathbf{p}_{j}\right)+1\right)$. Il était d'usage de remplacer le maximum par son majorant ${ }^{\dagger}$. Mais cette majoration devient très maladroite lorsque le groupe $\mathbf{G}_{j}$ est un tore ou une variété abélienne. Dans le premier cas, par définition de $h$, on a

$$
\max _{0 \leq s \leq \mathfrak{a}}\left\{h\left(s \mathbf{p}_{j}\right)\right\}=\max \left\{h\left(\mathbf{p}_{j}^{s}\right)\right\}=\max \left\{|s| h\left(\mathbf{p}_{j}\right)\right\}=\mathfrak{a} h\left(\mathbf{p}_{j}\right)
$$

et, dans le second cas, la hauteur $h$ est comparable à la hauteur de Néron-Tate $\widehat{h}$ (relative à une polarisation) de la variété abélienne (i.e. $h-\widehat{h}=O(1))$ et on a donc

$$
\max _{0 \leq s \leq \mathfrak{a}}\left\{h\left(s \mathbf{p}_{j}\right)\right\}=\mathfrak{a}^{2} \widehat{h}\left(\mathbf{p}_{j}\right)+O(1) .
$$

Dans ces deux cas, nous évitons un terme $\mathfrak{a}^{\rho_{j}}$ supplémentaire et cette absence peut se révéler vraiment importante lorsque (par exemple) $\mathbf{p}_{j}$ est un point de torsion du groupe $\mathbf{G}_{j}$.

${ }^{*}$ Supposons qu'il existe une fonction $b: \mathbb{R}^{+} \rightarrow \mathbb{R}^{+}$telle que $b(x) / x \underset{x \rightarrow \infty}{\longrightarrow} 0$ et telle que

$$
\log |\Lambda| \geq-b\left(h\left(1: u_{0}: \beta_{1}: \cdots: \beta_{d}\right)\right) .
$$

Alors le passage de $\left(u_{0}, \beta_{1}, \ldots, \beta_{d}\right)$ à $\left(\frac{u_{0}}{N}, \frac{\beta_{1}}{N}, \ldots, \frac{\beta_{d}}{N}\right)$ (où $N$ est un entier naturel $>0$, premier à l'idéal fractionnaire $\left(u_{0}\right)+\left(\beta_{1}\right)+\cdots+\left(\beta_{d}\right)$ de $\left.\mathcal{O}_{\mathbf{L}}\right)$ ne modifie pas le degré du corps de nombres $\mathbf{L}$ (ni, non plus, aucune des autres données) et transforme $\log |\Lambda|$ en $\log |\Lambda|-\log N$ ainsi que $h\left(1: u_{0}: \beta_{1}: \cdots: \beta_{d}\right)$ en $h\left(1: u_{0}: \beta_{1}: \cdots: \beta_{d}\right)+\log N$ (pour $\left.N>\max \left\{\left|\overline{u_{0}}\right|, \ldots,\left|\overline{\beta_{d}}\right|\right\}\right)$. La contradiction arrive lorsque $N \rightarrow \infty$.

${ }^{\dagger}$ Ce qui est source d'une erreur extrêmement fréquente dans la littérature $[45,46,65]$, erreur déjà constatée par G. DiAz [34], qui consistait à majorer $h\left(\mathbf{p}_{j}\right)+1$ par une quantité $\log V_{j}$, définie comme un majorant de $\max \left\{\frac{1}{D}, h\left(\mathbf{p}_{j}\right), \frac{\left\|\mathbf{u}_{j}\right\|^{\rho_{j}}}{D}\right\}$ au lieu de $\max \left\{1, h\left(\mathbf{p}_{j}\right), \frac{\left\|\mathbf{u}_{j}\right\|^{\rho_{j}}}{D}\right\}$. 
En l'absence d'informations particulières sur le point p de $\mathbf{G}(\overline{\mathbb{Q}})$, il convient de remplacer, dans les inégalités (2) et (3), le terme $\operatorname{card}\left(\Gamma_{\mathbf{p}}(\mathfrak{a})\right)$ par $\mathfrak{a}+1$. Cependant, lorsque $\mathbf{p}$ est un point de torsion de $\mathbf{G}(\overline{\mathbb{Q}})$ (ou, encore mieux, si $\mathbf{p}=0$, i.e. le logarithme $\mathbf{u}$ appartient au sous-groupe des périodes de $\mathbf{G}(\mathbb{C})$ ), le cardinal de $\Gamma_{\mathbf{p}}(\mathfrak{a})$ est, a priori, très inférieur à $\mathfrak{a}+1$ et, concurremment avec la remarque précédente, les inégalités (2) et (3) deviennent beaucoup plus précises. Si $\mathbf{u} \in \Omega_{\mathbf{G}(\mathbb{C})}$, il n'est pas exclu qu'une modification de la construction de la fonction auxiliaire ou qu'un lemme de Schwarz idoine améliore fortement ces inégalités.

Nous n'imposons aucune majoration de E, contrairement à [30] et [45]. C'est une question de présentation (voir remarque 2.9 de [45]), mais nous pensons que cela clarifie le lien entre les données et les paramètres. L'origine du paramètre $E$ est analytique. Il représente un quotient de rayons dans le lemme de Schwarz (approché).

Le calcul explicite des constantes qui apparaissent dans les théorèmes I.3.1 et I.3.2 nécessite la connaissance des données suivantes :

a) Pour chaque plongement $\sigma: \mathbf{K} \hookrightarrow \mathbb{C}$, les constantes $c_{\sigma}^{-}$et $c_{\sigma}^{+}$, définies par les inégalités (9) et (10), qui permettent de mesurer la « taille analytique »du plongement $\Phi$ par rapport à la norme choisie sur $t_{\mathbf{G}_{\sigma}}(\mathbb{C})$.

b) Les constantes liées à l'écriture explicite des formules d'additions et de dérivations dans le groupe $\mathbf{G}$ (page 16).

c) La constante de la proposition I.9.2. Cette constante peut être calculée pour un choix raisonnable des normes sur l'espace tangent et de $\Phi$ en reprenant les calculs de [8] et en s'appuyant en particulier sur la remarque 3 de cet article.

d) La hauteur d'un élément primitif $\alpha$ de $\mathbf{K}$ (i.e. tel que $\mathbf{K}=\mathbb{Q}(\alpha)$ ).

e) La constante qui apparaît dans la majoration du rang du système linéaire (lemme I.11.1). Cependant, un plongement « à la Serre », comme le nôtre, permet de prendre $8^{d}$ pour cette constante (voir le lemme 6.7 de [65]).

f) La constante $r$ liée à l'arithmétique du groupe $\mathbf{G}$ et qui permet d'avoir un modèle lisse $\mathcal{G} \rightarrow \operatorname{Spec} \mathcal{O}_{\mathbf{K}}[r]$ de $\mathbf{G}$ (lemme I.5.1). Lorsque $\mathbf{G}$ est une variété semi-abélienne, nous pourrions prendre $r=1$ en utilisant un modèle de Néron de $\mathbf{G}$, dont l'existence est établie dans [68].

Les constantes les plus difficiles à appréhender sont celles liées au plongement $\Phi$. Le mieux est donc d'éviter de plonger le groupe dans un espace projectif! C'est ce que nous avons fait dans la seconde partie de cette thèse (où les constantes sont donc explicites).

Le terme $D h\left(\xi_{1}: \cdots: \xi_{D}\right)$, qui apparaît dans les inégalités (2), (3) et (4), résulte de la construction du polynôme auxiliaire avec une variante du lemme de Siegel. En fait, ce terme est récurrent dans la littérature (voir, par exemple, le paragraphe 5 de [82] (condition (5.5)), le début de la démonstration de la proposition 3.8 de [81] (page 275), et bien sûr [1, 30, 45, $46,65,66])$ mais il ne représentait pas une contrainte réelle car, la plupart du temps *, il était inclus dans un terme général plus grand. Dans les cas favorables où la méthode des pentes (ou des déterminants d'interpolation) s'applique, cette quantité est supprimée (voir seconde partie). Lorsque le corps de nombres $\mathbf{L}$ est choisi « minimal » (corps de définition de $\mathbf{G}, W$ et $\mathbf{p})$, il existe une base $\xi_{1}, \ldots, \xi_{D}$ de $\mathbf{L}$ telle que la quantité $D h\left(\xi_{1}: \cdots: \xi_{D}\right)$ soit contrôlée.

\footnotetext{
*Sauf pour certaines mesures d'approximations simultanées (voir $[46,66])$.
} 
En effet, notons $\left(\beta_{0}^{(\ell)}: \cdots: \beta_{N_{\ell}}^{(\ell)}\right)$ l'image du point $\mathbf{p}_{\ell}$ par $\Phi$ dans $\mathbb{P}^{N_{\ell}}(\mathbf{L})(0 \leq \ell \leq n)$ et $\mathbf{K}_{\ell}$ le corps engendré sur $\mathbf{K}$ par les éléments $\beta_{j}^{(\ell)}, 0 \leq j \leq N_{\ell}$. Soit $\alpha$ un élément primitif de $\mathbf{K}_{\mid \mathbb{Q}}$. L'ensemble

$$
\left\{\alpha^{h} \beta_{1}^{i_{1}} \cdots \beta_{d}^{i_{d}} \prod_{\ell=0}^{n} \prod_{j=0}^{N_{\ell}}\left(\beta_{j}^{(\ell)}\right)^{m_{\ell, j}}\right\}
$$

où $i_{1}+\cdots+i_{d} \leq\left[\mathbf{K}\left(\beta_{1}, \ldots, \beta_{d}\right): \mathbf{K}\right], \sum_{j=0}^{N_{\ell}} m_{\ell, j} \leq\left[\mathbf{K}_{\ell}: \mathbf{K}\right]$ et $h \leq[\mathbf{K}: \mathbb{Q}]$, est un ensemble de générateurs du $\mathbb{Q}$-espace vectoriel $\mathbf{L}$. En particulier, $D$ étant le degré $[\mathbf{L}: \mathbb{Q}]$, on peut choisir une base $\xi_{1}=1, \xi_{2}, \ldots, \xi_{D}$ parmi ces générateurs. Cette base vérifie :

$$
h\left(\xi_{1}: \cdots: \xi_{D}\right) \leq\left[\mathbf{K}\left(\beta_{1}, \ldots, \beta_{d}\right): \mathbf{K}\right] h\left(1: \beta_{1}: \cdots: \beta_{d}\right)+\sum_{\ell=0}^{n}\left[\mathbf{K}_{\ell}: \mathbf{K}\right] h\left(\mathbf{p}_{\ell}\right)+[\mathbf{K}: \mathbb{Q}] h(\alpha)
$$

et donc

$$
h\left(\xi_{1}: \cdots: \xi_{D}\right) \leq D\left(h(W)+h\left(u_{0}\right)+h\left(\mathbf{p}_{1}\right)+\cdots+h\left(\mathbf{p}_{n}\right)\right)+[\mathbf{K}: \mathbb{Q}] h(\alpha) .
$$

Si cette majoration permet d'avoir une idée de la taille maximale de $D h\left(\xi_{1}: \cdots: \xi_{D}\right)$, il serait maladroit de la substituer systématiquement à ce terme (par exemple si $\mathbf{L}=\mathbb{Q}$ ). L'utilisation du lemme de Bombieri-Vaaler [13] au lieu du lemme de Thue-Siegel doit permettre de remplacer la quantité $D h\left(\xi_{1}: \cdots: \xi_{D}\right)$ par le logarithme de la valeur absolue du discriminant (absolue) de $\mathbf{L}$.

Enfin, il est très probable que ce résultat correctement adapté reste valide lorsque $W$ est un sous-espace vectoriel de codimension $>1$ de $t_{\mathrm{G}}(\overline{\mathbb{Q}})$. Cette généralisation fera l'objet d'une prochaine étude.

Afin de mieux percevoir la portée de la mesure (3) du théorème I.3.1, nous allons mentionner plusieurs conséquences (simples) de ce théorème, lorsque le groupe $\mathbf{G}$ est réduit à un unique groupe algébrique $(n=1)$, en isolant chacune des quantités $h(\mathbf{p}), D,\|\mathbf{u}\|$. Les données sont celles du $\S$ I.2, sans hypothèse particulière sur la nature du groupe algébrique $\mathbf{G}$.

Conséquence I.3.3 (Hauteur du point p). Il existe une constante

$$
c_{8}=c_{8}\left(\mathbf{G}, \Phi, \mathrm{d},\|\mathbf{u}\|, u_{0}, W, D\right)
$$

indépendante de $h(\mathbf{p})$, telle que si $\mathbf{u} \notin W$ alors

$$
\log \left|u_{0}-\beta_{1} u_{1}-\cdots-\beta_{d} u_{d}\right| \geq-c_{8} \frac{\max \{1, h(\mathbf{p})\}^{d}}{(\log \max \{e, h(\mathbf{p})\})^{d-1}} .
$$

Question : Quelle est la minoration optimale en $h(\mathbf{p})$ ? Une fonction linéaire de $h(\mathbf{p})$ ?

Conséquence I.3.4 (Degré du corps de nombres L). Il existe une constante

$$
c_{9}=c_{9}(\mathbf{G}, \Phi, \mathrm{d},\|\mathbf{u}\|, W, h(\mathbf{p})),
$$

indépendante de $D$, telle que si $\mathbf{u} \notin W$ alors

$$
\log \left|u_{0}-\beta_{1} u_{1}-\cdots-\beta_{d} u_{d}\right| \geq-c_{9} \frac{D^{2 d+2}}{(\log \max \{e, D\})^{d-1}} .
$$


Conséquence I.3.5 (Norme du logarithme u). Il existe une constante

$$
c_{10}=c_{10}(\mathbf{G}, \Phi, \mathrm{d}, W, D, h(\mathbf{p}))
$$

indépendante de $\|\mathbf{u}\|$, telle que si $\mathbf{u} \notin W$ alors

$$
\log \left|u_{0}-\beta_{1} u_{1}-\cdots-\beta_{d} u_{d}\right| \geq-c_{10} \max \{1,\|\mathbf{u}\|\}^{2 d}(\log \max \{e,\|\mathbf{u}\|\})^{d+2} .
$$

Les conséquences I.3.3 et I.3.5 améliorent, dans le cas général, les (meilleures) minorations (que nous avons déduites) de [45]:

$$
\log \left|u_{0}-\beta_{1} u_{1}-\cdots-\beta_{d} u_{d}\right| \geq-c_{11} \frac{\max \{1, h(\mathbf{p})\}^{d+1}}{(\log \max \{e, h(\mathbf{p})\})^{d}}
$$

et

$$
\log \left|u_{0}-\beta_{1} u_{1}-\cdots-\beta_{d} u_{d}\right| \geq-c_{12} \max \{1,\|\mathbf{u}\|\}^{2 d+2}(\log \max \{e, \| \mathbf{u} \mid\})^{d+1} .
$$

Ainsi, outre une mesure optimale en la hauteur de l'hyperplan, nous avons également amélioré la dépendance en la hauteur du point $\mathbf{p}$ et en la norme de son logarithme. La raison est que, au cours de la démonstration, nous avons remplacé la quantité $T \log T$ par $T \log \min \left\{D_{0}, T\right\}$ ( $T$ est un paramètre de dérivation et $D_{0}$ un degré partiel de polynôme). Comme le paramètre $T$ ne dépendait pas exclusivement de $h(W)$ (je pense, ici, à [30, 45, 65]), mais était aussi fonction de $h(\mathbf{p})$ et $\|\mathbf{u}\|$, la substitution du terme $T \log T$ au terme $T \log D_{0}$ apporte des modifications (en l'occurrence des améliorations) de la mesure. Ce n'est pas seulement $\log h(W)$ qui a disparu mais tout $\log \log B^{*}$. La dépendance en le degré du corps de nombres n'évolue pas. Le lecteur qui voudrait mieux comprendre ces points techniques est invité à se reporter au paragraphe A.8.

\section{$\S$ 3.c. Cas d'un groupe linéaire commutatif}

Dans ce paragraphe, nous détaillons le cas (classique) où $\mathbf{G}$ est le produit du groupe additif $\mathbb{G}_{\mathrm{a}}$ par un tore.

Corollaire I.3.6. Pour tout entier $k \geq 1$, il existe une constante $c(k)>0$ vérifiant la propriété suivante. Soient $u_{1}, \ldots, u_{k}$ des nombres complexes tels que $\alpha_{\ell}:=e^{u_{\ell}}$ appartienne $\grave{a} \overline{\mathbb{Q}}$ (pour tout $1 \leq \ell \leq k)$. Soient $u_{0}, \beta_{1}, \ldots, \beta_{k}$ des nombres algébriques non tous nuls. Soient $D$ le degré $d u$ corps de nombres $\mathbf{M}:=\mathbb{Q}\left(\alpha_{1}, \ldots, \alpha_{k}, u_{0}, \beta_{1}, \ldots, \beta_{k}\right)$ et $\xi_{1}, \ldots, \xi_{D}$ une base $d u$ $\mathbb{Q}$-espace vectoriel $\mathbf{M}$. Soit $E$ un réel $\geq$ e. Posons $\Lambda:=u_{0}+\beta_{1} u_{1}+\cdots+\beta_{k} u_{k}$,

$$
\mathfrak{a}^{\prime}:=1+\left[\frac{D \log \left(D+h\left(\alpha_{1}\right)+\cdots+h\left(\alpha_{k}\right)+E\left|u_{1}\right|+\cdots+E\left|u_{k}\right|\right)}{\log E}\right],
$$

et

$$
V:=\operatorname{card}\left(\Gamma_{\mathbf{p}}\left(\mathfrak{a}^{\prime}\right)\right) \times\left(D h\left(1: u_{0}: \beta_{1}: \cdots: \beta_{k}\right)+\mathfrak{a}^{\prime} \cdot \log E\right) \times \prod_{j=1}^{k}\left(1+\frac{D h\left(\alpha_{j}\right)+E\left|u_{j}\right|}{\log E}\right) .
$$

Premier cas : $\Lambda=0$.

Alors $u_{0}=0$ et il existe une $\mathbb{Z}$-base $e_{1}, \ldots, e_{k}$ de $\mathbb{Z}^{k}$, un entier naturel $0<q<k$ et des entiers $1 \leq j_{1}<\cdots<j_{k-q} \leq k$, tels que

${ }^{*}$ Cette phrase ésotérique est destinée aux happy few. 
a) Le $k$-uplet $\left(\beta_{1}, \ldots, \beta_{k}\right)$ appartient au $\mathbf{M}$-espace vectoriel engendré par $e_{1}, \ldots, e_{q}$.

b) Si $e_{\ell, 1}, \ldots, e_{\ell, k}$ désignent les coordonnées de $e_{\ell}$ dans la base canonique de $\mathbb{Z}^{k}$, alors, pour tout $\ell \in\{1, \ldots, q\}$, on $a e_{\ell, 1} u_{1}+\cdots+e_{\ell, k} u_{k}=0$.

c) Si $\mathcal{M}$ désigne la matrice $\left(e_{\ell, i}\right)_{\substack{1 \leq \ell \leq q \\ 1 \leq i \leq k}} \in \operatorname{Mat}_{q, k}(\mathbb{Z})$ alors

$$
\left(\operatorname{det} \mathcal{M}^{\mathrm{t}} \mathcal{M}\right)^{1 / 2} \leq c(k) \operatorname{card}\left(\Gamma_{\mathbf{p}}\left(\mathfrak{a}^{\prime}\right)\right) \times\left\{\prod_{\ell=1}^{k-q}\left(1+\frac{D h\left(\alpha_{j_{\ell}}\right)+E\left|u_{j_{\ell}}\right|}{\log E}\right)\right\} \times \max \left\{1, \frac{D h\left(\xi_{1}: \cdots: \xi_{D}\right)}{V}\right\}
$$

où ${ }^{\mathrm{M}} \mathcal{M}$ est la matrice transposée de $\mathcal{M}$.

Second cas : $\Lambda \neq 0$.

Alors on a

$$
\log |\Lambda| \geq-c(k) \max \left\{V, \operatorname{Dh}\left(\xi_{1}: \cdots: \xi_{D}\right)\right\}
$$

De plus, si $u_{0} \neq 0$ et

$$
V^{\prime}:=\mathfrak{a}^{\prime} \times\left(D h\left(1: \beta_{1}: \cdots: \beta_{k}\right)+\max _{0 \leq s \leq c(k) \mathfrak{a}^{\prime}}\left\{D h\left(s u_{0}\right)\right\}+\log E\right) \times \prod_{j=1}^{k}\left(1+\frac{D h\left(\alpha_{j}\right)+E\left|u_{j}\right|}{\log E}\right),
$$

alors

$$
\log |\Lambda| \geq-c(k) \max \left\{V^{\prime}, \operatorname{Dh}\left(\xi_{1}: \cdots: \xi_{D}\right)\right\} .
$$

Démonstration. La démonstration de ce corollaire à partir des théorèmes I.3.1 et I.3.2 repose essentiellement sur la structure des sous-groupes algébriques d'un groupe linéaire commutatif (voir, par exemple, la proposition 5.6 de [84], page 157).

- Si $\Lambda=0$, d'après le théorème I.3.1 appliqué à $\mathbf{G}=\mathbb{G}_{\mathrm{a}} \times \mathbb{G}_{\mathrm{m}}^{k}$, il existe un sous-groupe algébrique connexe $\widetilde{\mathbf{G}}$ de $\mathbf{G}$ vérifiant les points suivants :

a) Il existe un sous-groupe $\widetilde{Z}$ de $\mathbb{Z}^{k}$ tel que $\widetilde{\mathbf{G}}=\{0\} \times \mathbf{T}_{\widetilde{Z}}$, où, si $A$ est un anneau (commutatif), $\mathbf{T}_{\widetilde{Z}}(A)=\left\{\underline{x} \in A^{k} ; \underline{x} \underline{\underline{m}}=1\right.$ pour tout $\left.\underline{m} \in \widetilde{Z}\right\}$. Notons $q$ le rang de $\widetilde{Z}$. Soient $e_{1}, \ldots, e_{k}$ une base (adaptée à $\widetilde{Z}$ ) de $\mathbb{Z}^{k}$ et $a_{1}, \ldots, a_{q}$ des entiers naturels non nuls tels que $\widetilde{Z}:=\mathbb{Z} . a_{1} e_{1} \oplus \cdots \oplus \mathbb{Z}$. $a_{q} e_{q}$. Comme $\widetilde{\mathbf{G}}$ est connexe, le $\mathbb{Z}$-module $\widetilde{Z}$ est facteur direct dans $\mathbb{Z}^{k}$ (on dit aussi que $\widetilde{Z}$ est un sous-groupe primitif de $\mathbb{Z}^{k}$ ), i.e. $\mathbb{Z}^{k} / \widetilde{Z}$ est sans torsion. Par conséquent, les entiers $a_{i}$ sont égaux à 1 . Notons que la partie additive de $\widetilde{\mathbf{G}}$ est nulle car sinon on aurait $t_{\mathbb{G}_{\mathrm{a}}} \subseteq\left\{z_{0}=\beta_{1} z_{1}+\cdots+\beta_{k} z_{k}\right\}$. De plus, l'espace tangent $t_{\widetilde{\mathbf{G}}}=t_{\mathbf{T}_{\tilde{Z}}}$ s'identifie à l'ensemble

$$
\widetilde{Z}^{\perp}=\left\{\left(x_{1}, \ldots, x_{k}\right) \in \mathbf{M}^{k} ; x_{1} m_{1}+\cdots+x_{k} m_{k}=0 \text { pour tout }\left(m_{1}, \ldots, m_{k}\right) \in M\right\}
$$

(voir Lemma 8.13 de [84]). L'inclusion de $t_{\mathbf{T}_{\tilde{Z}}}$ dans l'hyperplan $W:=\left\{\beta_{1} z_{1}+\cdots+\beta_{k} z_{k}=\right.$ $0\}$ se traduit alors par $W^{\perp}=\mathbf{M} .\left(\beta_{1}, \ldots, \beta_{k}\right) \subseteq \widetilde{Z} \otimes \mathbf{M}$.

b) L'appartenance de $\left(u_{0}, u_{1}, \ldots, u_{k}\right)$ à $t_{\widetilde{\mathbf{G}}}(\mathbb{C})$ signifie précisément que $u_{0}=0$ et $e_{\ell, 1} u_{1}+$ $\cdots+e_{\ell, k} u_{k}=0$, pour tout $\ell \in\{1, \ldots, q\}$.

c) Le degré du groupe $\pi(\widetilde{\mathbf{G}})=\mathbf{T}_{\widetilde{Z}}$, relatif au plongement usuel de $\mathbf{G}$ dans $\left(\mathbb{P}^{1}\right)^{k+1}$, est, à une constante près ne dépendant que de $k$, égal à la somme des valeurs absolues des mineurs maximaux de la matrice $\mathcal{M}$ et est donc équivalent à $\sqrt{\operatorname{det}\left(\mathcal{M}^{\mathrm{t}} \mathcal{M}\right)}$, en vertu de la formule de Cauchy-Binet (pour plus de détails, on peut se reporter à la démonstration de la proposition 4 de [8]). L'inégalité (5) résulte alors de la majoration (2) de $\operatorname{deg} \pi(\widetilde{\mathbf{G}})$. 
- Si $\Lambda \neq 0$, les minorations de $\log |\Lambda|$ sont les conséquences directes des inégalités (3) et (4).

Ce résultat entraîne le théorème célèbre de BAKER :

Théorème (BAKER, 1966). Si $\alpha_{1}, \ldots, \alpha_{k}$ sont des nombres algébriques dont les logarithmes sont linéairement indépendants sur $\mathbb{Q}$ alors $1, \log \alpha_{1}, \ldots, \log \alpha_{k}$ sont linéairement indépendants sur $\overline{\mathbb{Q}}$.

La majoration du déterminant de la première partie du corollaire I.3.6 s'inscrit dans le prolongement des travaux sur les relations de dépendance linéaire entre les logarithmes de nombres algébriques. Elle est une conséquence de la démonstration de la mesure d' indépendance linéaire. Pour obtenir des « petites » relations de dépendance linéaire, il est parfois plus efficace d'attaquer le problème directement (donc sans fonction auxiliaire, extrapolation, ....) à l'aide, par exemple, de la géométrie des nombres (voir lemme 7.19. de [84], page 222). Ce problème est lié aux relations de dépendance multiplicative entre nombres algébriques et à l'étude du covolume du sous-groupe de $\mathbb{Z}^{k}$ :

$$
\left\{\left(b_{1}, \ldots, b_{k}\right) \in \mathbb{Z}^{k} ; \alpha_{1}^{b_{1}} \cdots \alpha_{k}^{b_{k}}=1\right\}
$$

Le majorant du covolume est de la forme

$$
c(k, \mathbf{L}) \times \max _{1 \leq j_{1}<\cdots<j_{k-q} \leq k}\left\{\prod_{\ell=1}^{k-q} h\left(\alpha_{j_{\ell}}\right)\right\} .
$$

Un théorème plus précis se trouve page 205 de [7] (voir également [5]).

Par ailleurs, le corollaire I.3.6 ne contient pas les résultats quantitatifs déjà connus pour les sous-groupes linéaires commutatifs (comme, par exemple, le théorème 9.1 de [84]), ne serait-ce que parce que, contrairement à nous, les auteurs précisent la constante $c(k)$. En fait, dans le cas non-homogène $\left(u_{0} \neq 0\right)$, le terme

$$
\mathfrak{a}^{\prime} \times\left(D h\left(1: \beta_{1}: \cdots: \beta_{k}\right)+\max _{s}\left\{D h\left(s u_{0}\right)\right\}+\log E\right)
$$

devrait plutôt être remplacé par

$$
\operatorname{Dh}\left(1: u_{0}: \beta_{1}: \cdots: \beta_{k}\right)+\mathfrak{a}^{\prime}+\log E
$$

( [84], theorem 9.1, page 251). L'explication de cette anomalie provient d'une utilisation (encore) trop « grossière » du lemme de zéros de Philippon, car, contrairement à [84], nous n'avons pas supposé que les logarithmes $u_{1}, \ldots, u_{k}$ étaient linéairement indépendants* sur $\mathbb{Q}$. A fortiori, avec la mesure d'indépendance (6), nous ne retrouvons pas les mesures fines relatives au « cas rationnel homogène », qui concerne les minorations de $\left|b_{1} \log \alpha_{1}+\cdots+b_{k} \log \alpha_{k}\right|$ avec $b_{i} \in \mathbb{Q}$ et $\alpha_{j} \in \overline{\mathbb{Q}}$ (le groupe algébrique sous-jacent étant $\mathbb{G}_{\mathrm{m}}^{k}$ ou $\mathbb{G}_{\mathrm{a}}^{k-1} \times \mathbb{G}_{\mathrm{m}}$ selon que l'on utilise la méthode de Baker ou de Schneider, voir [84], chapitre 14.4), ces mesures étant obtenues à partir de méthodes et d'outils spécifiques comme les polynômes de Fel'dman.

${ }^{*}$ Cette hypothèse supplémentaire permet, par exemple, de calculer le cardinal des quotients $\left(\Gamma_{\mathbf{p}}(S)+\right.$ $\left.\mathbf{G}^{\prime}(\mathbf{L})\right) / \mathbf{G}^{\prime}(\mathbf{L})$ qui apparaissent si on utilise le lemme de zéros [63]. 


\section{$\S 4$. Organisation du texte}

La démonstration des théorèmes I.3.1 et I.3.2 fait intervenir l'existence de modèles lisses des groupes $\mathbf{G}_{j}$ et de plongements de ces groupes dans des espaces projectifs, plongements qui possèdent des propriétés supplémentaires « à la Serre ». Ces outils de géométrie algébrique sont détaillés dans le paragraphe suivant. Ensuite vient la preuve des théorèmes I.3.1 et I.3.2. Tout d'abord (§ I.6), nous « justifions » le choix des données de départ (en particulier l'hyperplan $W$, transversal aux espaces tangents $t_{\mathbb{G}_{\mathrm{a}}}$ et $\left.t_{\mathbf{G}_{1}} \oplus \cdots \oplus t_{\mathbf{G}_{n}}\right)$. Nous choisissons alors des paramètres de manière à ce que la section d'un fibré ample sur $\mathbf{G}$, qui sera construite avec ces paramètres, ne puisse s'annuler le long de l'hyperplan avec un ordre de multiplicité élevé en les premiers multiples d'un point $\mathbf{p}\left(:=\exp _{\mathbf{G}(\mathbb{C})}(\mathbf{u})\right)$ sans être identiquement nulle (§ I.7). Le choix de ces paramètres est guidé par le souci de prendre en compte d'emblée le « sous-groupe obstructeur » fourni par le lemme de zéros de PHILIPPon [63]. La contrainte liée à ce type de méthode est que, dans le reste de la démonstration, nous devons garder (jusqu'à l'extrapolation analytique) un sous-groupe obstructeur particulier qui conditionne le type d'extrapolation effectué au § I.13, selon qu'un multiple de $\mathbf{p}$ appartienne ou non à ce sous-groupe (distinction entre cas périodique et cas non-périodique, fin du paragraphe I.7). Dans le cas périodique (qui est, de loin, le plus délicat), nous donnons quelques informations complémentaires sur le sous-groupe obstructeur, qui seront à l'origine du théorème I.3.1 (§ I.9). Après quelques énoncés techniques concernant le choix de bases pour l'hyperplan (§ I.8 et I.9.b) et les modifications des dérivées liées au passage d'une base à une autre (§ I.10), nous entrons dans le vif de la démonstration avec la construction de la fonction auxiliaire (§ I.11 et I.12) puis nous extrapolons «à la Baker » (dans le cas non-périodique seulement, le cas périodique subissant un traitement différent). Un paramétrage local directement lié au groupe algébrique et non plus à son espace tangent nous permet d'apporter une amélioration essentielle dans l'estimation arithmétique de la première dérivée non-nulle de la fonction auxiliaire. Le résultat du paragraphe I.14 est au centre de la démonstration des théorèmes du $\S$ I.3.a, qui découle alors d'un raisonnement par l'absurde. Enfin, en annexe, nous reprenons la démonstration des théorèmes I.3.1 et I.3.2 sans fixer les paramètres. Nous justifions alors le choix des paramètres effectué au paragraphe I.7.

\section{$\S 5$. Modèle du groupe algébrique}

Les estimations ultramétriques du paragraphe I.14 sont à l'origine du besoin que nous aurons de modèles entiers (i.e. sur $\mathcal{O}_{\mathbf{K}}$ ) et lisses des groupes $\mathbf{G}_{j}$. Nous présentons ici les propriétés que nous pouvons attendre d'un tel modèle, en particulier vis-à-vis de la représentation de la loi d'addition du groupe dans un espace projectif. Ce passage par la géométrie algébrique (et en particulier la notion de schéma) permet de nous affranchir des contraintes techniques liées à la construction effective de paramètres locaux, comme l'ont fait S. DAVID et N. Hirata-Kohno dans [31] pour les courbes elliptiques ou, dans un contexte différent et dans le cas d'une variété abélienne, comme le fait P. GRAFtiEAux dans sa thèse [38]. La méthode de pentes permet d'utiliser ce formalisme de manière plus raffinée en évitant le plongement du groupe dans un espace projectif.

Résultats généraux. Soit $\mathbf{H}$ un groupe algébrique commutatif, défini sur un corps de nombres 
$\mathbf{K}$, de dimension $d$.

Lemme I.5.1. Il existe un entier $r^{-1}$ et un schéma en groupes

$$
\mathcal{H} \longrightarrow \operatorname{Spec} \mathcal{O}_{\mathbf{K}}[r]
$$

lisse et quasi-projectif, dont la fibre générique coüncide avec $\mathbf{H}$. De plus, l'entier $r^{-1}$ peut être choisi de telle façon que l'anneau $A:=\mathcal{O}_{\mathbf{K}}[r]$ soit principal.

Démonstration. Pour l'existence de $\mathcal{H}$, nous renvoyons à [22].

Pour la principalité de $A$, c'est un résultat bien connu, mais faute de références précises, nous en proposons une démonstration :

Considérons $\left\{\overline{\mathfrak{P}}_{1}, \ldots, \overline{\mathfrak{P}}_{h}\right\}$ le groupe des classes d'idéaux de $\mathcal{O}_{\mathbf{K}}$. Chaque $\mathfrak{P}_{i}^{h}$ est un idéal principal ; choisissons $r^{\prime-1}$ le produit des normes (sur $\mathbf{K}$ ) des $\mathfrak{P}_{i}^{h}$ et de l'entier $r^{-1}$ choisi pour la construction de $\mathcal{H}$. Alors $\mathfrak{P}_{i} \mathcal{O}_{\mathbf{K}}\left[r^{\prime}\right]=(1)\left(\operatorname{car} \mathfrak{P}_{i}^{h} \mathcal{O}_{\mathbf{K}}\left[r^{\prime}\right]=(1)\right)$. Soit $I$ un idéal de $\mathcal{O}_{\mathbf{K}}\left[r^{\prime}\right]$. On a $I=\left(I \cap \mathcal{O}_{\mathbf{K}}\right) \mathcal{O}_{\mathbf{K}}\left[r^{\prime}\right]$ (si $x \in I$, $r^{\prime-\alpha} x \in I \cap \mathcal{O}_{\mathbf{K}}$ pour un certain $\left.\alpha \in \mathbb{N}\right)$. Or tout idéal de $\mathcal{O}_{\mathbf{K}}$ est principal dans $\mathcal{O}_{\mathbf{K}}\left[r^{\prime}\right]$, car un tel idéal est équivalent (modulo un idéal principal) à un produit de puissances de $\mathfrak{P}_{i}$. Donc $I=\left(I \cap \mathcal{O}_{\mathbf{K}}\right) \mathcal{O}_{\mathbf{K}}\left[r^{\prime}\right]$ est principal. Et l'anneau $A:=\mathcal{O}_{\mathbf{K}}\left[r^{\prime}\right]$ convient.

Proposition I.5.2. Soit $\varepsilon: \operatorname{Spec} A \rightarrow \mathcal{H}$ la section nulle de $\mathcal{H}$, d'idéal de définition $\mathcal{J}$. Il existe un ouvert affine non-vide $\mathcal{U}$ de $\varepsilon(\operatorname{Spec} A)$, il existe d sections $s_{1}, \ldots, s_{d}$ de $\mathcal{J}$ sur $\mathcal{U}$ telles que $\mathbf{e}:=\left(\varepsilon^{*} s_{1}, \ldots, \varepsilon^{*} s_{d}\right)$ forme une base de $t_{\mathcal{H}}$ et telle que l'application $T_{i} \rightarrow s_{i}$ définisse un isomorphisme de schémas formels :

$$
\widehat{\mathcal{H}} \stackrel{\sim}{\longrightarrow} \operatorname{Specf} A\left[\left[T_{1}, \ldots, T_{d}\right]\right]
$$

où $\widehat{\mathcal{H}}$ est le complété formel de $\mathcal{H}$ le long de la section nulle.

Démonstration. C'est la proposition 2.9 (page 19) de la thèse de P. Graftieaux [38].

Considérons $\sigma: \mathbf{K} \hookrightarrow \mathbb{C}$ un plongement de $\mathbf{K}$ dans $\mathbb{C}$. La quasi-projectivité de $\mathcal{H}$ donne l'existence d'un plongement de $A$-schémas $i: \mathcal{H} \hookrightarrow \mathbb{P}_{A}^{N}$ qui induit $i_{\sigma}: \mathcal{H}_{\sigma}(\mathbb{C}) \hookrightarrow \mathbb{P}^{N}(\mathbb{C})$. D'après [73], le groupe de Lie $\mathcal{H}_{\sigma}(\mathbb{C})$ admet alors une représentation de son exponentielle $\exp _{\sigma}$ par des fonctions holomorphes ${ }^{*} \varphi_{0}, \ldots, \varphi_{N}$ sur $t_{\mathcal{H}_{\sigma}}(\mathbb{C})$ :

$$
\forall z \in t_{\mathcal{H}_{\sigma}}(\mathbb{C}), \quad \exp _{\sigma}(z)=\left(\varphi_{0}(z): \cdots: \varphi_{N}(z)\right) .
$$

Nous pouvons choisir ces fonctions $\varphi_{j}$ avec les propriétés suivantes ( $c f$. [83], en particulier les propriétés 4.4 et 4.6 , page 76$)$ :

- Les fonctions $\varphi_{j}$ sont des fonctions analytiques d'ordre $\rho$ inférieur ou égal à 2 et sans zéro commun dans $t_{\mathcal{H}_{\sigma}}(\mathbb{C})$.

- $\left(\varphi_{0}(0), \cdots, \varphi_{N}(0)\right)=(1,0, \ldots, 0)$

- Soit $\|.\|_{\sigma}$ la norme hermitienne sur $t_{\mathcal{H}_{\sigma}}(\mathbb{C})$ qui rend orthonormée la base e définie dans l'énoncé du corollaire I.5.2. Il existe deux constantes positives $c_{\sigma}^{-}$et $c_{\sigma}^{+}$telles que, pour tout $z \in t_{\mathcal{H}_{\sigma}}(\mathbb{C})$, on ait

$$
\exp \left\{-c_{\sigma}^{-}\left(1+\|z\|_{\sigma}\right)^{\rho}\right\} \leq \max \left\{\left|\varphi_{0}(z)\right|, \ldots,\left|\varphi_{N}(z)\right|\right\}
$$

et

$$
\max \left\{\left|\varphi_{0}(z)\right|, \ldots,\left|\varphi_{N}(z)\right|\right\} \leq \exp \left\{c_{\sigma}^{+}\left(1+\|z\|_{\sigma}\right)^{\rho}\right\} .
$$

*Pour ne pas alourdir excessivement les notations, nous omettrons la référence au plongement $\sigma$; dans le contexte, il n'y aura aucune confusion possible. 
- Il existe une constante $c_{\mathcal{H}_{\sigma}}^{(1)}$ vérifiant : pour tout $x \in t_{\mathcal{H}_{\sigma}}(\mathbb{C})$, il existe un voisinage $\mathcal{V}_{x}$ de 0 dans $t_{\mathcal{H}_{\sigma}}(\mathbb{C})$ et $N+1$ polynômes $A_{0}, \ldots, A_{N}$ de $\mathbf{K}[\mathbf{X}, \mathbf{Y}]$, où $\mathbf{X}=\left(X_{0}, \ldots, X_{N}\right)$ et $\mathbf{Y}=\left(Y_{0}, \ldots, Y_{N}\right)$, bi-homogènes, tous de même bidegré, de degré total $\leq c_{\mathcal{H}_{\sigma}}^{(1)}$, tels que, pour tout $z \in \mathcal{V}_{x}$, les éléments

$$
\left(\varphi_{0}(z+x): \cdots: \varphi_{N}(z+x)\right)
$$

et

$$
\left(A_{0}(\varphi(z), \varphi(x)): \cdots: A_{N}(\varphi(z), \varphi(x))\right)
$$

soient égaux.

- Identifions $t_{\mathcal{H}_{\sigma}}(\mathbb{C})$ à $\mathbb{C}^{d}$ grâce à la base e. Il existe une constante $c_{\mathcal{H}_{\sigma}}^{(2)}$ vérifiant : pour tout triplet $(k, j, h)$ avec $k \in\{1, \ldots, d\}$ et $j, h \in\{0, \ldots, N\}$, il existe un polynôme $C_{k j h} \in \mathbf{K}[\mathbf{X}]$, de degré total $\leq c_{\mathcal{H}_{\sigma}}^{(2)}$, tel que

$$
\frac{\partial}{\partial z_{k}}\left(\frac{\varphi_{j}}{\varphi_{h}}\right)=C_{k j h}\left(\frac{\varphi_{0}}{\varphi_{h}}, \ldots, \frac{\varphi_{N}}{\varphi_{h}}\right)
$$

Nous désignerons par $\Phi$ l'application holomorphe qui, à $z \in t_{\mathcal{H}_{\sigma}}(\mathbb{C})$, associe

$$
\left(\varphi_{0}(z), \ldots, \varphi_{N}(z)\right) \in \mathbb{C}^{N+1}
$$

et, pour $h \in\{0, \ldots, N\}$, par $\psi_{h}$ l'application méromorphe

$$
\left(\frac{\varphi_{0}}{\varphi_{h}}, \ldots, \frac{\varphi_{N}}{\varphi_{h}}\right)
$$

\section{Notations.}

Lorsque $\mathbf{H}$ est un groupe produit $\mathbf{H}_{1} \times \cdots \times \mathbf{H}_{\ell}$ de $\mathbf{K}$-groupes algébriques (commutatifs), les résultats précédents s'appliquent à chacun des groupes $\mathbf{H}_{i}$, et en prenant par exemple le rationnel $r=r_{1} \cdots r_{\ell}$, le schéma en groupes $\mathcal{H}:=\mathcal{H}_{1} \times \cdots \times \mathcal{H}_{\ell}$ est lisse sur $\operatorname{Spec} \mathcal{O}_{\mathbf{K}}[r]$. Après le choix d'un plongement $\sigma: \mathbf{K} \rightarrow \mathbb{C}$, l'exponentielle complexe de $\mathbf{H}_{i, \sigma}(\mathbb{C})$ sera représenté par les fonctions $\varphi_{h}^{(i)}$ avec $0 \leq h \leq N_{i}$. La fonction $\varphi_{h}^{(i)}$ est d'ordre $\leq \rho_{i}$. Nous noterons également $A_{h}^{(i)}$ (resp. $C_{k j h}^{(i)}$ ) les polynômes provenant des lois d'additions de $\mathbf{H}_{i}$ (resp. des propriétés différentielles de $\left.\mathbf{H}_{i}\right)$. Ce sont des polynômes en les variables $\mathbf{X}_{i}:=\left(X_{0}^{(i)}, \ldots, X_{N_{i}}^{(i)}\right)$ et $\mathbf{Y}_{i}:=\left(Y_{0}^{(i)}, \ldots, Y_{N_{i}}^{(i)}\right)$. Enfin, nous désignerons par $\psi_{h}^{(i)}: t_{\mathbf{H}_{i, \sigma}}(\mathbb{C}) \rightarrow \mathbb{C}^{N_{i}+1}$ l'application méromorphe

$$
\left(\frac{\varphi_{0}^{(i)}}{\varphi_{h}^{(i)}}, \ldots, \frac{\varphi_{h-1}^{(i)}}{\varphi_{h}^{(i)}}, 1, \frac{\varphi_{h+1}^{(i)}}{\varphi_{h}^{(i)}}, \ldots, \frac{\varphi_{N_{i}}^{(i)}}{\varphi_{h}^{(i)}}\right)
$$

Nous noterons $\|\cdot\|_{j, \sigma}$ (ou $\|\cdot\|_{j}$ si $\sigma$ est le plongement $\sigma_{0}$ de $\mathbf{K}$ dans $\mathbb{C}$ choisi au $\S$ I.2.a) la norme hermitienne sur $t_{\mathcal{H}_{j, \sigma}}(\mathbb{C})$ obtenue comme précédemment; $\|\cdot\|_{\sigma}$ sera la norme induite $\operatorname{sur} t_{\mathcal{H}_{\sigma}}(\mathbb{C})$.

\section{Démonstration des théorèmes I.3.1 et I.3.2}




\section{$\S 6$. Réductions}

N. Hirata-Kohno a remarqué [45] que l'ajout d'un groupe affine $\mathbb{G}_{\mathrm{a}}$ à $\mathbf{G}$ a un rôle modérateur sur les dérivations. L'adjonction « artificielle» de ce facteur $\mathbb{G}_{\mathrm{a}}$ à $\mathbf{G}$ revient à minorer $\left|1.0-\left(\beta_{1} u_{1}+\cdots+\beta_{d} u_{d}\right)\right|$. La présence de ce facteur $\mathbb{G}_{\mathrm{a}}$ contrebalance l'influence négative des dérivations sur les coefficients $\beta_{i}$. En effet, dériver, à l'ordre $T$, le long de l'hyperplan $z_{0}=\beta_{1} z_{1}+\cdots+\beta_{d} z_{d}$, la fonction $P \circ \exp _{\left(\mathbb{G}_{\mathrm{a}} \times \mathbf{G}\right)}$ (où $P$ est un polynôme multihomogène), au point 0 , équivaut à dériver $F_{W}(\underline{z}):=P\left(\beta_{1} z_{1}+\cdots+\beta_{d} z_{d}, \exp _{\mathbf{G}}\left(z_{1} e_{1}+\cdots+z_{d} e_{d}\right)\right.$ ) (la notation ici se veut plus suggestive que rigoureuse) dans toutes les directions $\frac{\partial}{\partial z_{i}}(i=1, \ldots, d)$, ce qui fait apparaître, dans les coefficients de Taylor de $F_{W}$ d'ordre $T$, une dépendance polynomiale en les $\beta_{i}$ de degré majoré par min $\left\{\operatorname{deg}_{X_{0}} P, T\right\}$ (au lieu de $T$ ).

Si cette astuce est relativement simple du point de vue technique, il est plus difficile d'en percevoir la philosophie. Certes le nouveau groupe $\mathbb{G}_{\mathrm{a}}$ s'accompagne d'un paramètre $D_{0}$, « degré de liberté » supplémentaire - choisi indépendamment de $h(W)$ - qui augmente la possibilité de résoudre le système de paramètres. Cependant, en général, il est plutôt néfaste (en particulier pour la dépendance en le degré $D$ du corps de nombres) d'accroître la dimension du groupe algébrique, d'autant qu'avec la méthode employée, chaque nouveau groupe donne un facteur multiplicatif supplémentaire à la mesure de dépendance (voir commentaire ci-dessous).

Nous procéderons en fait de la manière suivante :

Tout d'abord, nous pouvons supposer, sans perte de généralité, qu' aucun des espaces tangents $t_{\mathbf{G}_{i}}$ n'est inclus dans $W$ (i.e. que la famille des $\beta_{j}$ correspondant aux variables de $t_{\mathbf{G}_{i}}$ n'est pas réduite à $\{0\})$.

Sans perte de généralités, nous supposerons que $\max \left\{\left|u_{0}\right|,\left|\beta_{m}\right|_{1 \leq m \leq d}\right\}=1$. En effet, si on note $\mathfrak{x}$ un élément de $\left\{u_{0}\right\} \cup\left\{\beta_{m}\right\}_{1 \leq m \leq d}$, de valeur absolue maximale, alors

$$
D h\left(u_{0}\right)+D h(W) \geq \log |\mathfrak{x}| \geq-D h\left(u_{0}\right)-D h(W)
$$

et il est donc équivalent de minorer $\log |\Lambda|$ et $\log \left|\frac{u_{0}}{\mathfrak{x}}-\sum_{m=1}^{d}\left(\frac{\beta_{m}}{\mathfrak{x}}\right) \cdot u_{m}\right|$.

Soient $r$ un rationnel $>0$ et $\mathcal{G}=\mathcal{G}_{0} \times \cdots \times \mathcal{G}_{n}$ un modèle lisse de $\mathbf{G}$ sur $\mathcal{O}_{\mathbf{K}}[r]$ vérifiant les conditions du paragraphe précédent. Nous noterons $\mathbf{e}=\left(e_{0}, \ldots, e_{d}\right)$ la base de $t_{\mathcal{G}}$ obtenue par recollement des bases de $t_{\mathcal{G}_{i}}$, apparaissant dans la proposition I.5.2 ( $e_{0}$ est la base canonique de $\left.t_{\mathcal{G}_{0}}\right)$. Chaque schéma en groupes $\mathcal{G}_{\ell}$ se plonge dans un espace projectif $\mathbb{P}_{\mathcal{O}_{\mathbf{K}}[r]}^{N_{\ell}}$. Nous noterons $\mathbb{P}$ l'espace multiprojectif $\mathbb{P}^{N_{0}} \times \cdots \times \mathbb{P}^{N_{n}}$. L'espace tangent à l'origine de $\mathcal{G}$ est naturellement muni de normes hermitiennes $\|\cdot\|_{\sigma}$ aux places infinies $\sigma$, comme cela est expliqué au paragraphe précédent.

Enfin, avec les notations de la fin du paragraphe I.5, nous noterons $\Phi$ l'application analytique de $t_{\mathbf{G}}(\mathbb{C})$ dans $\mathbb{C}^{\left(N_{0}+1\right)+\cdots+\left(N_{n}+1\right)}$ qui à $z$ associe

$$
\left(\left(\varphi_{0}^{(0)}(z), \ldots, \varphi_{N_{0}}^{(0)}(z)\right), \ldots,\left(\varphi_{0}^{(n)}(z), \ldots, \varphi_{N_{n}}^{(n)}(z)\right)\right) .
$$

Commentaire heuristique.

La méthode générale et les outils techniques locaux employés dans ce texte pour démontrer les théorèmes font qu'à chaque facteur $\mathbf{G}_{j}$ du groupe $\mathbf{G}$ correspond un facteur (multiplicatif) de la mesure de dépendance, ce facteur étant, grosso modo,

$$
\left(D \max _{0 \leq s \leq S}\left\{h\left(s \mathbf{p}_{j}\right)\right\}+\left(E S\left\|\mathbf{u}_{j}\right\|\right)^{\rho_{j}}\right)^{\delta_{j}}
$$


(pour $1 \leq j \leq n$ ). Donc, si on veut rendre la mesure de dépendance optimale, le mieux est de décomposer au maximum le groupe $\mathbf{G}$ (et, le cas échéant, de «comprimer » les facteurs $\mathbb{G}_{\mathrm{a}}$ pour diminuer la dimension du groupe). En effet, considérer, par exemple, $\mathbf{G}$ comme un groupe d'un seul tenant conduirait à une mesure de dépendance comportant au moins un terme de la forme

$$
\left(D \max _{0 \leq s \leq S}\{h(s \mathbf{p})\}+(E S\|\mathbf{u}\|)^{\rho}\right)^{d}
$$

(où $\rho:=\max \left\{\rho_{i}\right\}$ et $\left.\mathbf{p}:=\exp _{\mathbf{G}(\mathbb{C})} \mathbf{u}\right)$ au lieu du terme

$$
\prod_{j=1}^{n}\left(D \max _{0 \leq s \leq S}\left\{h\left(s \mathbf{p}_{j}\right)\right\}+\left(E S\left\|\mathbf{u}_{j}\right\|\right)^{\rho_{j}}\right)^{\delta_{j}},
$$

qui est nettement meilleur, compte tenu de $h(\mathbf{p}) \approx h\left(\mathbf{p}_{1}\right)+\cdots+h\left(\mathbf{p}_{k}\right)$ et $\|\mathbf{u}\| \approx\left\|\mathbf{u}_{1}\right\|+\cdots+\left\|\mathbf{u}_{k}\right\|$.

Ce principe de « dévissage total » du groupe $\mathbf{G}$ est freiné par la possibilité pour les $\mathbf{G}_{j}$ d'être des extensions (non triviales) de groupes. Cependant, si on écrit une suite de décomposition

$$
0 \longrightarrow \mathbb{G}_{\mathrm{a}}^{d_{0}} \times \mathbb{G}_{\mathrm{m}}^{d_{1}} \longrightarrow \mathbf{G} \longrightarrow A \longrightarrow 0
$$

(où $A$ est une variété abélienne), les espaces tangents $t_{\mathbf{G}}$ et $t_{\mathbb{G}_{\mathrm{a}}^{d_{0}}} \oplus t_{\mathbb{G}_{\mathrm{m}}^{d_{1}}} \oplus t_{A}$ sont $K$-isomorphes (non canoniquement), et si on décompose $A$, à son tour, à isogénie près, en $A_{1}^{e_{1}} \times \cdots \times A_{\mathfrak{l}}^{e_{\mathfrak{l}}}$, où les $A_{i}$ sont des variétés abéliennes simples (théorème de Poincaré), l'espace $t_{\mathbf{G}}$ est isomorphe* à $t_{\mathbb{G}_{\mathrm{a}}^{d_{0}}} \oplus t_{\mathbb{G}_{\mathrm{m}}^{d_{1}}} \oplus\left(t_{A_{1}} \oplus \cdots \oplus t_{A_{1}}\right) \oplus \cdots \oplus\left(t_{A_{\mathrm{l}}} \oplus \cdots \oplus t_{A_{\mathrm{l}}}\right)$. Comme le théorème I.3.1 porte sur des objets $(\mathbf{u} \text { et } W)^{m}$ de cet espace tangent, tout reviendrait, en quelque sorte, à obtenir une minoration en travaillant avec le groupe $\mathbf{G}^{\prime}=\mathbb{G}_{\mathrm{a}}^{d_{0}} \times \cdots \times A_{\mathfrak{l}}^{e_{\mathfrak{l}}}$. La seule difficulté est de s'assurer que le point $\mathbf{u}^{\prime}$ dans $t_{\mathbb{G}_{\mathrm{a}}} d_{0} \oplus \cdots \oplus t_{A_{\mathrm{l}}}$, correspondant à $\mathbf{u}$, peut être choisi de manière à avoir une exponentielle algébrique, et que, dans ce cas, la hauteur de $\exp _{\mathbf{G}^{\prime}}\left(\mathbf{u}^{\prime}\right)$ est du même ordre de grandeur que celle de $\exp _{\mathbf{G}}(\mathbf{u})$. Cette obstruction est, en général, impossible à lever dans la mesure où le scindage des espaces tangents ne respecte pas l'algébricité des données.

\section{$\S 7$. Choix des paramètres}

Soit $C_{0}$ un réel positif et $E \geq e$. Au long de la démonstration, nous dirons souvent qu'une inégalité est vraie « pour $C_{0}$ assez grand ». Cela signifie qu'il existe une constante ne dépendant que de $(\mathbf{G}, \Phi)$ telle que si $C_{0}$ est supérieur à cette constante alors l'inégalité est vraie. Dans le contexte, la constante en question sera facile à calculer (voir commentaires du $\S$ I.3.a) et, comme la preuve ne génère qu'un nombre fini de telles constantes, le réel $C_{0}$ peut être choisi comme le maximum de ces constantes.

Définissons

$$
S:=\left[C_{0}^{5} \mathfrak{a}\right], \quad S_{0}:=\left[C_{0}^{3} \mathfrak{a}\right] .
$$

On pourra noter que $D \log S \leq S \log E$ pour $C_{0}$ assez grand. Pour définir les paramètres suivants, nous aurons à distinguer le cas général du cas où $\prod_{j=1}^{n} \mathbf{G}_{j}$ est une variété semi-

${ }^{*}$ Et, comme la mesure de dépendance laisse indéfinie la constante qui dépend de $\mathbf{G}$, nous pouvons rendre isométrique cet isomorphisme. 
abélienne et $u_{0} \neq 0$. Rappelons que $\mathbf{p}:=\exp _{\mathbf{G}_{\sigma_{0}}(\mathbb{C})} \mathbf{u}$ et posons :

$$
\begin{aligned}
U_{0}:=\max \{ & C_{0}^{4 d-1} \times \frac{\operatorname{card}\left(\Gamma_{\mathbf{p}}(S)\right)}{(S \log E)^{d}} \times\left(D h(W)+D h\left(u_{0}\right)+S \log E\right) \\
& \left.\times \prod_{j=1}^{n}\left(S \log E+\max _{0 \leq s \leq(d+1) S}\left\{D h\left(s \mathbf{p}_{j}\right)\right\}+\left(E S\left\|\mathbf{u}_{j}\right\|\right)^{\rho_{j}}\right)^{\delta_{j}}, C_{0} D h\left(\xi_{1}: \cdots: \xi_{D}\right)\right\}
\end{aligned}
$$

resp.

$$
\begin{aligned}
U_{0}^{\prime}:=\max \{ & C_{0}^{4 d-1} \frac{S}{(S \log E)^{d}}\left(D h(W)+\max _{0 \leq s \leq(d+1) S}\left\{D h\left(s u_{0}\right)\right\}+C_{0}^{2} \log E\right) \\
& \left.\times \prod_{j=1}^{n}\left(S \log E+\max _{0 \leq s \leq(d+1) S}\left\{D h\left(s \mathbf{p}_{j}\right)\right\}+\left(E S\left\|\mathbf{u}_{j}\right\|\right)^{\rho_{j}}\right)^{\delta_{j}}, C_{0} D h\left(\xi_{1}: \cdots: \xi_{D}\right)\right\} .
\end{aligned}
$$

Le réel $U_{0}$ (resp. $\left.U_{0}^{\prime}\right)$ est comparable, à une fonction polynomiale de $C_{0}$ près, à la mesure du théorème I.3.1 (resp. théorème I.3.2). Pour ne pas alourdir la démonstration, nous ne traiterons que le cas général avec $U_{0}$. Il va de soi que si nous sommes dans le cas particulier où $u_{0} \neq 0$ et $\prod_{j=1}^{n} \mathbf{G}_{j}$ est une variété abélienne alors $U_{0}$ doit être remplacé par $U_{0}^{\prime}$ (et de même pour les paramètres définis ci-après). Le lecteur pourra s'ôter d'un doute, sur ce qui s'apparente à une friponnerie, en consultant l'annexe, où les calculs sont écrits (et les propositions énoncées) avec des paramètres quelconques. Nous insistons sur le fait que la légère amélioration de la mesure d'indépendance dans le cas particulier sus-nommé n'a été rendue possible que grâce à la condition (plus faible) exigée par le lemme de zéros (voir démonstration du corollaire I.11.2).

Notons alors

$$
\begin{gathered}
\widetilde{T}:=\frac{C_{0}^{4} U_{0}}{S \log E}, \quad\left(\text { resp. } \widetilde{T}:=\frac{C_{0}^{4} U_{0}^{\prime}}{S \log E}\right) \\
\widetilde{T}_{0}:=\frac{\widetilde{T}}{C_{0}^{2}}, \\
\widetilde{D}_{0}:=\frac{U_{0}}{D h(W)+D h\left(u_{0}\right)+S \log E}, \\
\left(r e s p . \quad \widetilde{D}_{0}:=\frac{U_{0}^{\prime}}{D h(W)+\max _{0 \leq s \leq(d+1) S}\left\{D h\left(s u_{0}\right)\right\}+C_{0}^{2} \log E}\right)
\end{gathered}
$$

et, pour $j \in\{1, \ldots, n\}$,

$$
\begin{gathered}
\widetilde{D}_{j}:=\frac{U_{0}}{S \log E+\max _{0 \leq s \leq(d+1) S}\left\{D h\left(s \mathbf{p}_{j}\right)\right\}+\left(E S\left\|\mathbf{u}_{j}\right\|\right)^{\rho_{j}}}, \\
\text { resp. } \left.\widetilde{D}_{j}:=\frac{U_{0}^{\prime}}{S \log E+\max _{0 \leq s \leq(d+1) S}\left\{D h\left(s \mathbf{p}_{j}\right)\right\}+\left(E S\left\|\mathbf{u}_{j}\right\|\right)^{\rho_{j}}}\right) .
\end{gathered}
$$

On notera que les inégalités suivantes sont vérifiées : 
- $\widetilde{T}_{0} \geq C_{0} \cdot \max \left\{\widetilde{D}_{1}, \ldots, \widetilde{D}_{n}\right\}$.

- $\widetilde{T}_{0} \geq C_{0} \cdot \widetilde{D}_{0}$ dans le cas général.

- $\widetilde{T}_{0} \geq C_{0} \cdot \frac{\widetilde{D}_{0}}{S_{0}}$ dans le cas où $u_{0} \neq 0$ et $\prod_{j=1}^{n} \mathbf{G}_{j}$ est une variété semi-abélienne.

Nous supposerons que $\widetilde{D}_{1}, \ldots, \widetilde{D}_{n}$ sont rangés en ordre décroissant, i.e.

$$
\max _{0 \leq s \leq(d+1) S}\left\{D h\left(s \mathbf{p}_{1}\right)\right\}+\left(E S\left\|\mathbf{u}_{1}\right\|\right)^{\rho_{1}} \geq \max _{0 \leq s \leq(d+1) S}\left\{D h\left(s \mathbf{p}_{2}\right)\right\}+\left(E S\left\|\mathbf{u}_{2}\right\|\right)^{\rho_{2}} \geq \cdots
$$

Cette hypothèse, qui n'est pas du tout essentielle (ni réductrice), simplifie la présentation de la majoration du degré du sous-groupe obstructeur donnée au $\S$ I.9.a.

Soit $x \in \mathbb{R}^{+}$. Notons également $D_{i}^{\#}:=x \widetilde{D}_{i}, D_{i}:=\left[D_{i}^{\#}\right], T:=[\widetilde{T}]$ et $T_{0}:=\left[\widetilde{T}_{0}\right]$.

La proposition suivante, qui détermine le choix de $x$, s'énonce en fonction de la partie homogène de plus haut degré du polynôme de Hilbert-Samuel d'une sous-variété $V$ de $\mathbb{P}^{N_{0}} \times$ $\cdots \times \mathbb{P}^{N_{n}}$, polynôme que nous notons $\mathscr{H}$. Nous n'aurons besoin que de propriétés immédiates de $\mathscr{H}$ (liées à sa structure de polynôme homogène) sauf au § I.9.a.

Proposition I.7.1. Il existe un réel $x \in] 0,1]$ tel que les conditions suivantes soient vérifiées.

(i) Pour tout sous-groupe algébrique connexe $\mathbf{G}^{\prime}$ de $\mathbf{G}_{\mathbf{L}}$ de codimension $r^{\prime}$, dont l'espace tangent à l'origine est inclus dans l'hyperplan $W$, on a :

$$
(\widetilde{T})^{r^{\prime}-1} \times \operatorname{card}\left(\frac{\Gamma_{\mathbf{p}}(S)+\mathbf{G}^{\prime}(\mathbf{L})}{\mathbf{G}^{\prime}(\mathbf{L})}\right) \times \mathscr{H}\left(\mathbf{G}^{\prime} ; D_{0}^{\#}, \ldots, D_{n}^{\#}\right) \geq C_{0} \mathscr{H}\left(\mathbf{G} ; D_{0}^{\#}, \ldots, D_{n}^{\#}\right) .
$$

(ii) Il existe au moins un sous-groupe algébrique algébrique connexe $\widetilde{\mathbf{G}}$ qui réalise l'égalité.

Fixons $\widetilde{\mathbf{G}}$ et notons $\widetilde{d}$ (resp. $\widetilde{r}$ ) sa dimension (resp. sa codimension dans $\mathbf{G}$ ).

Démonstration. La démonstration est classique (depuis [65]). Cependant, pour simplifier la vérification des théorèmes, nous reproduisons la preuve.

Soit $\mathbf{G}^{\prime}$ un sous-groupe algébrique connexe de $\mathbf{G}_{\mathbf{L}}$; définissons

$$
A\left(\mathbf{G}^{\prime}\right):=(\widetilde{T})^{r^{\prime}-1} \times \operatorname{card}\left(\frac{\Gamma_{\mathbf{p}}(S)+\mathbf{G}^{\prime}(\mathbf{L})}{\mathbf{G}^{\prime}(\mathbf{L})}\right) \times \frac{\mathscr{H}\left(\mathbf{G}^{\prime} ; \widetilde{D}_{0}, \ldots, \widetilde{D}_{n}\right)}{C_{0} \mathscr{H}\left(\mathbf{G} ; \widetilde{D}_{0}, \ldots, \widetilde{D}_{n}\right)}
$$

et $B\left(\mathbf{G}^{\prime}\right):=A\left(\mathbf{G}^{\prime}\right)^{1 / r^{\prime}} \times \max \left\{1, A\left(\mathbf{G}^{\prime}\right)\right\}^{\frac{r^{\prime}-1}{r^{\prime}}}$. Comme nous avons (c'est un encadrement trivial)

$$
\left(\operatorname{deg}_{\Phi} \mathbf{G}^{\prime}\right) \max _{\substack{0 \leq i_{k} \leq \delta_{k} \\ i_{0}+\cdots+i_{n}=d^{\prime}}}\left\{\widetilde{D}_{0}^{i_{0}} \cdots \widetilde{D}_{n}^{i_{n}}\right\} \geq \mathscr{H}\left(\mathbf{G}^{\prime} ; \widetilde{D}_{0}, \ldots, \widetilde{D}_{n}\right) \geq\left(\operatorname{deg}_{\Phi} \mathbf{G}^{\prime}\right) \min _{\substack{0 \leq i_{k} \leq \delta_{k} \\ i_{0}+\cdots+i_{n}=d^{\prime}}}\left\{\widetilde{D}_{0}^{i_{0}} \cdots \widetilde{D}_{n}^{i_{n}}\right\}
$$

on en déduit

$$
C_{0} \widetilde{T} \times\left(\frac{\max _{0 \leq i \leq n}\left\{\widetilde{D}_{i}\right\}}{\widetilde{T}}\right)^{r^{\prime}} \geq \frac{\operatorname{deg}_{\Phi} \mathbf{G}^{\prime}}{A\left(\mathbf{G}^{\prime}\right)} \geq \frac{C_{0} \widetilde{T}}{S+1} \times\left(\frac{\max _{0 \leq i \leq n}\left\{\widetilde{D}_{i}\right\}}{\widetilde{T}}\right)^{r^{\prime}} .
$$

Ainsi, à degré (de $\mathbf{G}^{\prime}$ ) fixé, la quantité $A\left(\mathbf{G}^{\prime}\right)$ (et donc aussi $B\left(\mathbf{G}^{\prime}\right)$ ) ne prend qu'un nombre fini de valeur et lorsque $\operatorname{deg}_{\Phi} \mathbf{G}^{\prime}$ s'accroît indéfiniment, il en est de même de $A\left(\mathbf{G}^{\prime}\right)$. Ces observations justifient l'existence d'un sous-groupe connexe $\widetilde{\mathbf{G}}$ de $\mathbf{G}_{\mathbf{L}}$ tel que $B(\widetilde{\mathbf{G}})$ soit minimal parmi 
tous les $\mathbf{G}^{\prime}$ d'espace tangent (à l'origine) inclus dans $W$. Posons alors $x:=B(\widetilde{\mathbf{G}})$ (clairement non nul).

Montrons que $x \leq 1$.

Le choix des paramètres permet de calculer

$$
\begin{aligned}
A(\{0\}) & =(\widetilde{T})^{d} \times \operatorname{card}\left(\Gamma_{\mathbf{p}}(S)\right) \times \frac{1}{C_{0} \times\left(\operatorname{deg}_{\Phi} \mathbf{G}\right) \times \widetilde{D}_{0}^{\delta_{0}} \cdots \widetilde{D}_{n}^{\delta_{n}}} \\
& =\frac{1}{\operatorname{deg}_{\Phi} \mathbf{G}}
\end{aligned}
$$

et, donc, $1 \geq A(\{0\})$, ce qui implique

$$
x=\min _{t_{\mathbf{G}^{\prime}} \subseteq W}\left\{B\left(\mathbf{G}^{\prime}\right)\right\} \leq B(\{0\})=A(\{0\})^{1 /(d+1)} \leq 1 .
$$

Montrons que l'inégalité (16) est vérifiée :

Posons

$$
\aleph_{\mathbf{G}^{\prime}}=(\widetilde{T})^{r^{\prime}-1} \times \operatorname{card}\left(\frac{\Gamma_{\mathbf{p}}(S)+\mathbf{G}^{\prime}(\mathbf{L})}{\mathbf{G}^{\prime}(\mathbf{L})}\right) \times \frac{\mathscr{H}\left(\mathbf{G}^{\prime} ; D_{0}^{\#}, \cdots, D_{n}^{\#}\right)}{C_{0} \cdot \mathscr{H}\left(\mathbf{G} ; D_{0}^{\#}, \cdots, D_{n}^{\#}\right)} .
$$

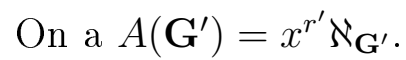

- Si $A\left(\mathbf{G}^{\prime}\right) \geq 1$, on a $A\left(\mathbf{G}^{\prime}\right) \geq x$ et donc $\aleph_{\mathbf{G}^{\prime}} \geq\left(\frac{1}{x}\right)^{r^{\prime}-1} \geq 1$.

- Si $A\left(\mathbf{G}^{\prime}\right)<1$, on a $B\left(\mathbf{G}^{\prime}\right)=A\left(\mathbf{G}^{\prime}\right)^{1 / r^{\prime}} \geq x$, donc $\left(\aleph_{\mathbf{G}^{\prime}}\right)^{1 / r^{\prime}} x \geq x$, i.e. $\aleph_{\mathbf{G}^{\prime}} \geq 1$.

En remplaçant les inégalités par des égalités $(x=B(\widetilde{\mathbf{G}})$ et nécessairement $A(\widetilde{\mathbf{G}}) \leq 1$ sinon $x=A(\widetilde{\mathbf{G}})$ ce qui contredit $x \leq 1)$, on obtient $\aleph_{\widetilde{\mathbf{G}}}=1$.

Tout au long de la démonstration, nous aurons à tenir compte du sous-groupe $\widetilde{\mathbf{G}}$ introduit dans cette proposition. Nous montrerons plus loin (corollaire I.11.2) que le choix de $x$ permet d'assurer qu'aucun des entiers $D_{0}, \ldots, D_{n}$ n'est nul.

Définition I.7.2. Nous dirons que nous sommes dans le cas périodique s'il existe $s \in\{1, \ldots, 2(d+$ $1) S\}$ tel que $s \mathbf{u} \in \Omega_{\mathbf{G}(\mathbb{C})}+t_{\widetilde{\mathbf{G}}}(\mathbb{C})$, et dans le cas non-périodique si un tel entier $s$ n'existe pas.

Le cas non-périodique se prêtera à une extrapolation sur les points et nous poserons

$$
\Upsilon:=\left\{(\underline{t}, s) \in \mathbb{N}^{d} \times \mathbb{N} ;|\underline{t}| \leq 2(d+1) T \text { et } 0 \leq s<S_{0}\right\} .
$$

Dans le cas périodique, nous extrapolerons sur les dérivées et nous poserons

$$
\Upsilon:=\left\{(\underline{t}, s) \in \mathbb{N}^{d} \times \mathbb{N} ;|\underline{t}| \leq 2(d+1) T, \quad t_{d}<T_{0} \text { et } 0 \leq s<2(d+1) S\right\} .
$$

Remarque : il serait plus naturel de majorer $|\underline{t}|$ (resp. s) par $2 T$ (resp. $2 S)$ au lieu de $2(d+1) T$ (resp. $2(d+1) S)$. Le facteur $d+1$ supplémentaire est présent par pure commodité dans l'application du lemme de zéros de Philippon.

Le cardinal de $\Upsilon$ se calcule aisément, via le

Lemme I.7.3. Si n, A, B sont des entiers naturels non nuls alors le cardinal de l'ensemble

$$
\left\{\left(x_{1}, \ldots, x_{n}\right) \in \mathbb{N}^{n} ; x_{n} \leq A \text { et } x_{1}+\cdots+x_{n} \leq A+B\right\}
$$

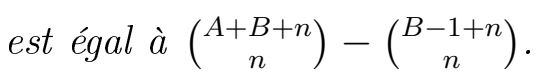




\section{Le cas général}

Démonstration. On a

$$
\begin{aligned}
\operatorname{card}\left\{\left(x_{1}, \ldots, x_{n}\right) ; x_{n} \leq A \text { et } x_{1}+\ldots+x_{n} \leq A+B\right\} \\
=\sum_{j=0}^{A+B} \operatorname{card}\left\{\left(x_{1}, \ldots, x_{n}\right) ; x_{n} \leq A \text { et } x_{1}+\ldots+x_{n}=j\right\} \\
=\sum_{j=0}^{A+B} \sum_{i=0}^{\min (A, j)} \operatorname{card}\left\{\left(x_{1}, \ldots, x_{n-1}\right) ; x_{1}+\ldots+x_{n-1}=j-i\right\} \\
=\sum_{j=0}^{A} \operatorname{card}\left\{\left(x_{1}, \ldots, x_{n-1}\right) ; x_{1}+\ldots+x_{n-1} \leq j\right\} \\
\quad+\sum_{j=A+1}^{A+B} \operatorname{card}\left\{\left(x_{1}, \ldots, x_{n-1}\right) ; x_{1}+\ldots+x_{n-1} \leq j\right\} \\
\quad-\sum_{j=A+1}^{A+B} \operatorname{card}\left\{\left(x_{1}, \ldots, x_{n-1}\right) ; x_{1}+\ldots+x_{n-1} \leq j-A-1\right\} \\
=\sum_{j=0}^{A+B} \operatorname{card}\left\{\left(x_{1}, \ldots, x_{n-1}\right) ; x_{1}+\ldots+x_{n-1} \leq j\right\}-\sum_{j=0}^{B-1} \operatorname{card}\left\{\left(x_{1}, \ldots, x_{n-1}\right) ; x_{1}+\ldots+x_{n-1} \leq j\right\} \\
=\sum_{j=B}^{A+B} \operatorname{card}\left\{\left(x_{1}, \ldots, x_{n-1}\right) ; x_{1}+\ldots+x_{n-1} \leq j\right\} \\
=\sum_{j=B}^{A+B}(j+n-1) \\
\quad j-1 \\
\end{aligned}
$$

et le lemme découle alors de la relation $\sum_{j=0}^{m}\left(\begin{array}{c}j+k \\ k\end{array}\right)=\left(\begin{array}{c}m+1+k \\ k+1\end{array}\right)$.

Nous en déduisons alors que

$$
\operatorname{card} \Upsilon=\left(\begin{array}{c}
2(d+1) T+d \\
d
\end{array}\right) \times S_{0}
$$

dans le cas non-périodique et

$$
\operatorname{card} \Upsilon=(2(d+1) S-1) \times\left[\left(\begin{array}{c}
2(d+1) T+d \\
d
\end{array}\right)-\left(\begin{array}{c}
2(d+1) T-T_{0}+d+1 \\
d
\end{array}\right)+1\right]
$$

dans le cas périodique. En fait, nous aurons seulement besoin de la majoration (assez grossière)

$$
\mu:=\operatorname{card} \Upsilon \leq 2(d+1) S(2(d+1) T+1)^{d} \leq c_{13} S T^{d} \leq e^{c_{14} U_{0}} .
$$

Les dérivées que nous considérerons seront toutes dans la direction de l'hyperplan $W$. Cependant, dans le cas périodique, nous choisirons une direction privilégiée (transcendante), selon laquelle nous extrapolerons.

\section{$\S 8$. Choix de bases pour l'hyperplan $W$}

Dans ce paragraphe très court, nous fixons une base orthonormée de l'hyperplan $W$, utile pour l'extrapolation «selon les dérivées » dans le cas périodique.

- Par définition, dans la base $\left(e_{0}, \ldots, e_{d}\right)$ de $t_{\mathbf{G}}$, l'hyperplan $W$ a pour équation $z_{0}=$ $\beta_{1} z_{1}+\cdots+\beta_{d} z_{d}$. La famille $\left\{\widetilde{e}_{i}=e_{i}+\beta_{i} e_{0}\right\}_{1 \leq i \leq d}$ constitue donc une base $\widetilde{\mathbf{e}}$ de $W$. 
- L'isomorphisme $\left(z_{1}, \ldots, z_{d}\right) \rightarrow z_{1} \widetilde{e}_{1}+\cdots+z_{d} \widetilde{e}_{d}$, entre $\mathbb{C}^{d}$ et $W \otimes \mathbb{C}$ (le produit tensoriel, ici, est relatif au plongement initial $\sigma_{0}$ de $\mathbf{L}$ dans $\mathbb{C}$ ), munit $W \otimes \mathbb{C}$ d'une (unique) structure hermitienne, héritée du produit scalaire canonique sur $\mathbb{C}^{d}$. Nous noterons |.| la norme ainsi obtenue* sur $W$, et nous poserons $\mathbf{e}^{\prime}:=\left(e_{1}^{\prime}, \ldots, e_{d}^{\prime}\right)$ une base orthonormée de $W$ pour ce produit scalaire, telle que $\left(e_{1}^{\prime}, \ldots, e_{\widetilde{d}}^{\prime}\right)$ soit également une base orthonormée de $t_{\widetilde{\mathbf{G}}}(\mathbb{C})$. Nous supposerons de plus que $\left(e_{\widetilde{d}+1}^{\prime}, \ldots, e_{d}^{\prime}\right)$ est ordonnée de telle façon que si $\mathbf{w}:=u_{1} \widetilde{e}_{1}+\cdots+u_{d} \widetilde{e}_{d}$ s'écrit $w_{1} e_{1}^{\prime}+\cdots+w_{d} e_{d}^{\prime}$ dans la base $\mathbf{e}^{\prime}$, alors

$$
\left|w_{d}\right|=\max \left\{\left|w_{i}\right| ; \quad \widetilde{d}+1 \leq i \leq d\right\} .
$$

Comme les bases $\widetilde{\mathbf{e}}$ et $\mathbf{e}^{\prime}$ de $W$ sont orthonormées pour le même produit hermitien sur $W \otimes \mathbb{C}$, les matrices de passages entre elles sont unitaires. On notera également que $\mathbf{u}-\mathbf{w}=\Lambda . e_{0}$ et donc $\|\mathbf{u}-\mathbf{w}\|=|\Lambda|$.

\section{$\S 9$. Le cas périodique : compléments}

Rappelons tout d'abord le résultat principal de [8] :

Théorème I.9.1. Soit $\mathfrak{G}$ un groupe algébrique défini sur $\mathbb{C}$, et $\iota$ un plongement (quasiprojectif) de $\mathfrak{G}$ dans un espace projectif $\mathbb{P}$. Lorsque $\mathfrak{G}^{\prime}$ est un sous-groupe algébrique de $\mathfrak{G}$, on note $\operatorname{deg}_{\iota} \mathfrak{G}^{\prime}$ le degré de l'adhérence de Zariski de $\iota\left(\mathfrak{G}^{\prime}\right)$ dans $\mathbb{P}$. On munit l'espace tangent à l'origine $t_{\mathfrak{G}}$ d'une distance (euclidienne) $\mathrm{d}$. Il existe une constante $c_{15}=c(\mathfrak{G}, \iota, \mathrm{d})$ telle que, pour tout sous-groupe algébrique $\mathfrak{G}^{\prime}$ de $\mathfrak{G}$, pour tout $x \in \Omega_{\mathfrak{G}}$, si $\mathrm{d}\left(x, t_{\mathfrak{G}^{\prime}}\right) \leq 1 /\left(c_{15} \mathrm{deg}_{\iota} \mathfrak{G}^{\prime}\right)$ alors $x \in \Omega_{\mathfrak{G}^{\prime}}$.

Nous déduisons alors la

Proposition 1.9.2. Il existe une constante $c_{16}$, ne dépendant que de $(\mathbf{G}, \Phi)$, telle que, si $\omega \in \Omega_{\mathbf{G}(\mathbb{C})} \backslash \Omega_{\widetilde{\mathbf{G}}(\mathbb{C})}$, on ait

$$
\mathrm{d}\left(\omega, t_{\widetilde{\mathbf{G}}}(\mathbb{C})\right) \geq \frac{1}{c_{16} \operatorname{deg}_{\Phi} \pi(\widetilde{\mathbf{G}})}
$$

où $\pi: \mathbf{G}(\mathbb{C}) \rightarrow \mathbf{G}_{1}(\mathbb{C}) \times \cdots \times \mathbf{G}_{n}(\mathbb{C})$ est la projection canonique.

Démonstration. Comme $\Omega_{\mathbb{G}_{\mathrm{a}}(\mathbb{C})}=\{0\}$, on a $\omega=\pi^{\prime}(\omega)$ où $\pi^{\prime}$ est la projection de $t_{\mathbf{G}}(\mathbb{C})$ sur $t_{\mathbf{G}_{1}(\mathbb{C}) \times \cdots \times \mathbf{G}_{n}(\mathbb{C})}$. Donc $\mathrm{d}\left(\omega, t_{\widetilde{\mathbf{G}}}(\mathbb{C})\right)=\mathrm{d}\left(\pi^{\prime}(\omega), t_{\widetilde{\mathbf{G}}}(\mathbb{C})\right)$. De plus, l'inclusion $\widetilde{\mathbf{G}} \subseteq \mathbb{G}_{\mathrm{a}} \times \pi(\widetilde{\mathbf{G}})$ implique $t_{\widetilde{\mathbf{G}}} \subseteq t_{\mathbb{G}_{\mathrm{a}}} \oplus t_{\pi(\widetilde{\mathbf{G}})}$ donc $\mathrm{d}\left(\pi^{\prime}(\omega), t_{\widetilde{\mathbf{G}}}(\mathbb{C})\right) \geq \mathrm{d}\left(\pi^{\prime}(\omega), t_{\pi(\widetilde{\mathbf{G}})}(\mathbb{C})\right)$. D'après le théorème I.9.1, on a

$$
\mathrm{d}\left(\pi^{\prime}(\omega), t_{\pi(\widetilde{\mathbf{G}})}(\mathbb{C})\right) \geq \frac{1}{c_{16} \operatorname{deg}_{\Phi} \pi(\widetilde{\mathbf{G}})},
$$

ce qui conclut la démonstration.

Des propriétés supplémentaires du groupe $\widetilde{\mathbf{G}}$, introduit au $\S$ I.7, seront obtenues à l'aide de cette proposition. Mais auparavant nous voulons majorer le degré de $\pi(\widetilde{\mathbf{G}})$.

*Dans le contexte, il ne devrait pas y avoir de confusion avec une « simple » valeur absolue. 


\section{$\S$ 9.a. Majoration $\operatorname{de} \operatorname{deg}_{\Phi} \pi(\widetilde{\mathbf{G}})$}

Proposition I.9.3. Soit $\mathscr{H}$ le polynôme multi-homogène (de Hilbert-Samuel) de G. Alors on $a$

$$
\mathscr{H}\left(\widetilde{\mathbf{G}} ; x_{0}, \ldots, x_{n}\right) \geq\left(\operatorname{deg}_{\Phi} \pi(\widetilde{\mathbf{G}})\right) \cdot \min \left\{x_{i_{1}} \cdots x_{i_{\tilde{d}}}\right\}
$$

où $\widetilde{d}=\operatorname{dim} \widetilde{\mathbf{G}}$ et le minimum porte sur les $\widetilde{d}$-uplets $\left(i_{1}, \ldots, i_{\widetilde{d}}\right)$ vérifiant $1 \leq i_{1} \leq \cdots \leq i_{\widetilde{d}} \leq n$ et chaque $i_{\ell}$ apparaissant au plus $\delta_{i_{\ell}}$ fois.

Remarque : l'hypothèse $t_{\widetilde{\mathbf{G}}} \subseteq W$ implique $\operatorname{dim} \pi(\widetilde{\mathbf{G}})=\widetilde{d}$ car la surjection $\widetilde{\mathbf{G}} \rightarrow \pi(\widetilde{\mathbf{G}})$ donne $\operatorname{dim} \pi(\widetilde{\mathbf{G}}) \leq \operatorname{dim} \widetilde{\mathbf{G}}$, et si cette inégalité était stricte, l'inclusion $\widetilde{\mathbf{G}} \subseteq \mathbf{G}_{0} \times \pi(\widetilde{\mathbf{G}})$ entrainerait $t_{\widetilde{\mathbf{G}}}=t_{\mathbf{G}_{0} \times \pi(\widetilde{\mathbf{G}})}=t_{\mathbf{G}_{0}} \oplus t_{\pi(\widetilde{\mathbf{G}})}$, et donc $t_{\mathbf{G}_{0}} \subseteq W$ ce qui est faux.

Démonstration. C'est immédiat d'après l'interprétation géométrique des coefficients du polynôme de Hilbert-Samuel (voir [63, 64]).

Lemme I.9.4. Soit $m$ le plus grand entier de $\{1, \ldots, n\}$ tel que $\delta_{1}+\cdots+\delta_{m-1} \leq \widetilde{d}$. Posons $\delta_{m}^{\prime}:=\widetilde{d}-\delta_{1}-\cdots-\delta_{m-1}$. Alors, le degré $\operatorname{deg}_{\Phi} \pi(\widetilde{\mathbf{G}})$ est majoré par

$$
\begin{aligned}
& C_{0}^{4 d+2} \times\left(\operatorname{deg}_{\Phi} \mathbf{G}\right) \times \frac{\operatorname{card}\left(\Gamma_{\mathbf{p}}(\mathfrak{a})\right)}{(\mathfrak{a} \log E)^{\tilde{d}}} \times \prod_{\ell=1}^{m-1}\left(\mathfrak{a} \log E+\max _{0 \leq s \leq(d+1) C_{0}^{5} \mathfrak{a}}\left\{D h\left(s \mathbf{p}_{\ell}\right)\right\}+\left(E \mathfrak{a}\left\|\mathbf{u}_{\ell}\right\|\right)^{\rho_{\ell}}\right)^{\delta_{\ell}} \\
& \times\left(\mathfrak{a} \log E+\max _{0 \leq s \leq(d+1) C_{0}^{5} \mathfrak{a}}\left\{D h\left(s \mathbf{p}_{m}\right)\right\}+\left(E \mathfrak{a}\left\|\mathbf{u}_{m}\right\|\right)^{\rho_{m}}\right)^{\delta_{m}^{\prime}} \times \max \left\{1, \frac{D h\left(\xi_{1}: \cdots: \xi_{D}\right)}{U}\right\} .
\end{aligned}
$$

Remarques :

$\boldsymbol{x}$ Cette inégalité précise la majoration (2) du théorème I.3.1 en montrant l'origine des entiers $j_{1}, \ldots, j_{m}$ via l'hypothèse (15), p. 20.

$\boldsymbol{x}$ En fait, pour la démonstration du théorème I.3.1, nous utiliserons plus couramment la majoration plus faible:

$$
\operatorname{deg}_{\Phi} \pi(\widetilde{\mathbf{G}}) \leq C_{0}^{20 d} \cdot\left(D+D h\left(\mathbf{p}_{1}\right)+\cdots+D h\left(\mathbf{p}_{n}\right)+E\left\|\mathbf{u}_{1}\right\|+\cdots+E\left\|\mathbf{u}_{n}\right\|\right)^{20 d},
$$

car c'est le logarithme de $\operatorname{deg}_{\Phi} \pi(\widetilde{\mathbf{G}})$ qui apparaittra.

Démonstration de ce lemme. La proposition I.9.3 et la définition de $\widetilde{\mathbf{G}}$ entraîne

$$
\begin{aligned}
\frac{C_{0}}{(\widetilde{T})^{\widetilde{r}-1} \operatorname{card}\left(\frac{\Gamma_{\mathbf{p}}(S)+\widetilde{\mathbf{G}}(\mathbf{L})}{\widetilde{\mathbf{G}}(\mathbf{L})}\right)} & =\frac{\mathscr{H}\left(\widetilde{\mathbf{G}} ; D_{0}^{\#}, \ldots, D_{n}^{\#}\right)}{\mathscr{H}\left(\mathbf{G} ; D_{0}^{\#}, \ldots, D_{n}^{\#}\right)} \\
& \geq \frac{\operatorname{deg}_{\Phi} \pi(\widetilde{\mathbf{G}})}{\operatorname{deg}_{\Phi} \mathbf{G}} \times \frac{1}{D_{0}^{\#} \max \left\{D_{j_{1}}^{\#} \cdots D_{\tilde{r}_{\tilde{r}-1}}^{\#}\right\}} \\
& =\frac{\operatorname{deg}_{\Phi} \pi(\widetilde{\mathbf{G}})}{\operatorname{deg}_{\Phi} \mathbf{G}} \times \frac{1}{D_{0}^{\#}\left(D_{n}^{\#}\right)^{\delta_{n}} \cdots\left(D_{\ell+1}^{\#}\right)^{\delta_{m+1}}\left(D_{\ell}^{\#}\right)^{\delta_{m}-\delta_{m}^{\prime}}}
\end{aligned}
$$

où $m$ est l'entier défini dans l'énoncé du lemme. Il s'ensuit

$$
\operatorname{deg}_{\Phi} \pi(\widetilde{\mathbf{G}}) \leq C_{0} \times\left(\operatorname{deg}_{\Phi} \mathbf{G}\right) \times \widetilde{D}_{0} \times \frac{\widetilde{D}_{n}^{\delta_{n}} \cdots \widetilde{D}_{m}^{\delta_{m}-\delta_{m}^{\prime}}}{\widetilde{T}^{\widetilde{r}-1}},
$$


ce qui, en remplaçant les paramètres par leur valeur, implique le résultat voulu. La seconde remarque qui suit le lemme est triviale. Le point important est que la majoration de $\operatorname{deg}_{\Phi} \pi(\widetilde{\mathbf{G}})$ est indépendante de $h(W)$.

Corollaire 1.9.5. Dans le cas périodique, supposons $\mathbf{u} \notin t_{\widetilde{\mathbf{G}}}(\mathbb{C})$. Alors, pour $C_{0}$ assez grand:

$$
\mathrm{d}\left(\mathbf{u}, t_{\widetilde{\mathbf{G}}}(\mathbb{C})\right) \geq C_{0}^{-21 d} \times\left(D+D\left(h\left(\mathbf{p}_{1}\right)+\cdots+h\left(\mathbf{p}_{n}\right)\right)+E\left\|\mathbf{u}_{1}\right\|+\cdots+E\left\|\mathbf{u}_{n}\right\|\right)^{-20 d}
$$

Et, toujours dans le cas périodique, supposons maintenant que

$$
|\Lambda|<\frac{1}{2} \mathrm{~d}\left(\mathbf{u}, t_{\widetilde{\mathbf{G}}}(\mathbb{C})\right) .
$$

Alors $\mathbf{w} \notin t_{\widetilde{\mathbf{G}}}(\mathbb{C})$ et $t_{\widetilde{\mathbf{G}}}(\mathbb{C}) \subsetneq W \otimes \mathbb{C}$. En particulier $w_{d} \neq 0$.

Démonstration. Soit $s_{0} \in\{1, \ldots, 2(d+1) S\}$ minimal tel que $s_{0} \mathbf{u} \in \Omega_{\mathbf{G}(\mathbb{C})}+t_{\widetilde{\mathbf{G}}}(\mathbb{C})$. On a donc $s_{0} \leq 2(d+1) \operatorname{card}\left(\frac{\Gamma_{\mathbf{p}}(S)+\widetilde{\mathbf{G}}(\mathbf{L})}{\widetilde{\mathbf{G}}(\mathbf{L})}\right)$. Comme $s_{0} \mathbf{u} \notin \Omega_{\widetilde{\mathbf{G}}(\mathbb{C})}\left(\right.$ sinon $\left.\mathbf{u} \in t_{\widetilde{\mathbf{G}}}(\mathbb{C})\right)$, la proposition I.9.2 et la majoration (22) impliquent l'inégalité voulue.

Par ailleurs, si w appartenait à $t_{\widetilde{\mathbf{G}}}(\mathbb{C})$, nous aurions $|\Lambda|=\|\mathbf{u}-\mathbf{w}\| \geq \mathrm{d}\left(\mathbf{u}, t_{\widetilde{\mathbf{G}}}(\mathbb{C})\right)$ ce qui contredit l'hypothèse. Comme $\mathbf{w} \in(W \otimes \mathbb{C}) \backslash t_{\widetilde{\mathbf{G}}}(\mathbb{C})$, on a $t_{\widetilde{\mathbf{G}}}(\mathbb{C}) \subsetneq W \otimes \mathbb{C}$, et par définition de $w_{d}\left(\S\right.$ I.8), on a $w_{d} \neq 0$.

Remarque : nous avons supposé $\mathbf{u} \notin t_{\widetilde{\mathbf{G}}}(\mathbb{C})$ alors qu'il serait plus « naturel » de se contenter de $\mathbf{u} \notin W \otimes \mathbb{C}$. Mais, si l'hypothèse du corollaire I.9.5 est fausse, nous disposons d'une précision supplémentaire sur $\mathbf{u}$.

\section{$\S$ 9.b. Nouvelle base pour $W \otimes \mathbb{C}$}

Dans le cas périodique, nous posons

$$
\mathbf{f}:=\left(e_{1}^{\prime}, \ldots, e_{d-1}^{\prime}, \mathbf{w}\right)
$$

(é⿱ $\mathbf{e}^{\prime}$ est la base orthonormée définie au $\S$ I.8). C'est une base de $W \otimes \mathbb{C}$ car $w_{d} \neq 0$. Pour unifier les notations, nous noterons également $\mathbf{f}:=\mathbf{e}^{\prime}$ dans le cas non-périodique.

Lemme I.9.6. Pour tout $x \in W$, on $a|x| \leq\|x\| \leq(\sqrt{d+1})|x|$.

Démonstration. Soit $x \in W$. On écrit

$$
\begin{aligned}
x & =x_{1} \widetilde{e}_{1}+\cdots+x_{d} \widetilde{e}_{d} \\
& =\left(\beta_{1} x_{1}+\cdots+\beta_{d} x_{d}\right) e_{0}+x_{1} e_{1}+\cdots+x_{d} e_{d}
\end{aligned}
$$

On a alors $|x|^{2}=\left|x_{1}\right|^{2}+\cdots+\left|x_{d}\right|^{2}$ et $\|x\|^{2}=\left|x_{1}\right|^{2}+\cdots+\left|x_{d}\right|^{2}+\left|\beta_{1} x_{1}+\cdots+\beta_{d} x_{d}\right|^{2}$.

Donc $|x| \leq\|x\|$ résulte des définitions et $\|x\| \leq(\sqrt{d+1})|x|$ vient du fait que les $\beta_{i}$ sont de module $\leq 1$, et de l'inégalité de Cauchy-Schwarz.

Proposition I.9.7. Dans le cas périodique, supposons que $|\Lambda|<\frac{1}{2} \mathrm{~d}\left(\mathbf{u}, t_{\widetilde{\mathbf{G}}}(\mathbb{C})\right)$. Alors on a

$$
\left|w_{d}\right| \geq \frac{1}{2(d+1)} \mathrm{d}\left(\mathbf{u}, t_{\widetilde{\mathbf{G}}}(\mathbb{C})\right) .
$$




\section{Le cas général}

Démonstration. Posons $x:=w_{1} e_{1}^{\prime}+\cdots+w_{\widetilde{d}} e_{\widetilde{d}}^{\prime}$. C'est un élément de $t_{\widetilde{\mathbf{G}}}(\mathbb{C})$ et $\mathbf{w}-x=w_{\widetilde{d}+1} e_{\widetilde{d}+1}^{\prime}+\cdots+$ $w_{d} e_{d}^{\prime}$. Donc $|\mathbf{w}-x| \leq(\sqrt{d-\widetilde{d}})\left|w_{d}\right| \leq(\sqrt{d+1})\left|w_{d}\right| \operatorname{car}\left|w_{d}\right|=\max _{\widetilde{d} \leq i \leq d}\left|w_{i}\right|$. Alors $\|\mathbf{w}-x\| \leq(d+1)\left|w_{d}\right|$ en vertu du lemme I.9.6, puis $\|\mathbf{u}-x\|-\|\mathbf{w}-\mathbf{u}\| \leq(d+1)\left|w_{d}\right|$, ce qui implique $\mathrm{d}\left(\mathbf{u}, t_{\widetilde{\mathbf{G}}}(\mathbb{C})\right)-|\Lambda| \leq$ $(d+1)\left|w_{d}\right|$. L'hypothèse permet de conclure.

Du corollaire I.9.5 et de la proposition I.9.7, nous déduisons le

Corollaire I.9.8. Sous les deux hypothèses ${ }^{*}$ du corollaire I.9.5, on a

$$
\left|w_{d}\right| \geq C_{0}^{-22 d} \times\left(D+D\left(h\left(\mathbf{p}_{1}\right)+\cdots+h\left(\mathbf{p}_{n}\right)\right)+E\left\|\mathbf{u}_{1}\right\|+\cdots+E\left\|\mathbf{u}_{n}\right\|\right)^{-20 d} .
$$

\section{$\S 10$. Changement de bases de dérivations}

L'objectif de ce paragraphe est de comparer les dérivées le long de $W$ d'une fonction analytique, lorsqu'on change la base de $W \otimes \mathbb{C}$, et de préciser le résultat dans les cas périodique et non-périodique. Nous aurons besoin du

Lemme I.10.1 (Variante du lemme 3.1 de [65]). Soient $\left(\mathrm{a}_{i, j}\right)_{\substack{1 \leq i \leq n \\ 1 \leq j \leq m}}$ des nombres complexes, $\mathrm{f}_{1}, \ldots, \mathrm{f}_{m}, z$ des vecteurs de $\mathbb{C}^{d+1}$, et $\Theta$ une fonction analytique complexe au voisinage de z. Posons $\mathrm{e}_{i}=\sum_{j=1}^{m} \mathrm{a}_{i, j} \mathrm{f}_{j}$ et $\mathrm{A}:=\sum_{i, j}\left|\mathrm{a}_{i, j}\right|$. Alors, pour tout entier naturel $T$, on a

$$
\max _{|\underline{t}|=T}\left\{\left|\frac{\mathcal{D}_{\mathrm{e}_{1}}^{t_{1}} \cdots \mathcal{D}_{\mathrm{e}_{n}}^{t_{n}}}{\underline{t} !} \Theta(z)\right|\right\} \leq \mathrm{A}^{T} \times \max _{|\underline{h}|=T}\left\{\left|\frac{\mathcal{D}_{\mathrm{f}_{1}}^{h_{1}} \cdots \mathcal{D}_{\mathrm{f}_{m}}^{h_{m}}}{\underline{h} !} \Theta(z)\right|\right\} .
$$

Démonstration. La démonstration consiste à développer $\frac{1}{t !} \mathcal{D}_{\mathrm{e}_{1}}^{t_{1}} \cdots \mathcal{D}_{\mathrm{e}_{n}}^{t_{n}}$ en utilisant la formule du multinôme :

$$
\frac{1}{\underline{t} !} \mathcal{D}_{\mathrm{e}_{1}}^{t_{1}} \cdots \mathcal{D}_{\mathrm{e}_{n}}^{t_{n}} \Theta(z)=\sum_{\substack{t_{i, 1}+\cdots+t_{i, m}=t_{i} \\ 1 \leq i \leq n}}\left(\prod_{i, j} \frac{\left(\mathrm{a}_{i, j}\right)^{t_{i, j}}}{\left(t_{i, j}\right) !}\right)\left\{\mathcal{D}_{\mathrm{f}_{1}}^{t_{1,1}+\cdots+t_{n, 1}} \cdots \mathcal{D}_{\mathrm{f}_{m}}^{t_{1, m}+\cdots+t_{n, m}} \Theta\right\}(z) .
$$

Le lemme se déduit alors des majorations

$$
\prod_{j=1}^{m}\left(t_{1, j}+\cdots+t_{n, j}\right) ! \leq\left(\sum_{i, j} t_{i, j}\right) !
$$

et

$$
\sum_{t_{i, 1}+\cdots+t_{i, m}=t_{i}}\left\{\left(\prod_{i, j} \frac{\left|\mathrm{a}_{i, j}\right|^{t_{i, j}}}{\left(t_{i, j}\right) !}\right) \times\left(\sum_{i, j} t_{i, j}\right) !\right\} \leq\left(\sum_{i, j}\left|\mathrm{a}_{i, j}\right|\right)^{|\underline{t}|} .
$$

Grâce à ce lemme appliqué aux bases $\mathbf{f}$ et $\widetilde{\mathbf{e}}$ de $W$ construites précédemment, nous obtenons la

*En fait, la seconde hypothèse suffit puisqu'elle implique la première. 
Proposition I.10.2. Soit $\Theta$ une fonction analytique au voisinage d'un point $z \in \mathbb{C}^{d+1}$. Soit $m$ un entier naturel.

- Dans le cas non-périodique :

$$
\frac{1}{d^{3 m / 2}} \cdot \max _{|\underline{t}| \leq m}\left\{\frac{1}{\underline{t} !}|\mathcal{D} \underset{\widetilde{\mathbf{e}}}{\underline{t}} \Theta(z)|\right\} \leq \max _{|\underline{t}| \leq m}\left\{\frac{1}{\underline{t} !}\left|\mathcal{D}_{\mathbf{f}}^{\underline{t}} \Theta(z)\right|\right\} \leq d^{3 m / 2} \cdot \max _{|\underline{t}| \leq m}\left\{\frac{1}{\underline{t} !}\left|\mathcal{D}_{\tilde{\widetilde{\mathbf{e}}}}^{\underline{t}} \Theta(z)\right|\right\}
$$

- Dans le cas périodique :

$$
\max _{|\underline{t}| \leq m}\left\{\frac{1}{\underline{t} !}\left|\mathcal{D}_{\widetilde{\mathbf{e}}}^{\underline{t}} \Theta(z)\right|\right\} \leq d^{m / 2}\left(d+\frac{1+\left|w_{1}\right|+\cdots+\left|w_{d-1}\right|}{\left|w_{d}\right|}\right)^{m} \max _{|\underline{\mid}| \leq m}\left\{\frac{1}{\underline{t} !}\left|\mathcal{D}_{\overline{\mathbf{f}}}^{\underline{t}} \Theta(z)\right|\right\}
$$

et

$$
\max _{|\underline{t}| \leq m}\left\{\frac{1}{\underline{t} !}\left|\mathcal{D}_{\mathbf{f}}^{\underline{t}} \Theta(z)\right|\right\} \leq d^{m / 2}(d+\|\mathbf{u}\|)^{m} \cdot \max _{|\underline{t}| \leq m}\left\{\frac{1}{\underline{t} !}\left|\mathcal{D}_{\tilde{\widetilde{\mathbf{e}}}}^{\underline{t}} \Theta(z)\right|\right\}
$$

Remarque : dans le cas périodique, on a :

$$
\begin{aligned}
& \frac{1+\left|w_{1}\right|+\cdots+\left|w_{d-1}\right|}{\left|w_{d}\right|} \\
& \leq 2(d+1)\left(\frac{\sqrt{d}}{2}+\frac{1+d^{1 / 2}\|\mathbf{u}\|}{\mathrm{d}\left(\mathbf{u}, t_{\widetilde{\mathbf{G}}}(\mathbb{C})\right)}\right) \\
& \leq C_{0}^{23 d}\left(D+D h\left(\mathbf{p}_{1}\right)+\cdots+D h\left(\mathbf{p}_{n}\right)+E\left\|\mathbf{u}_{1}\right\|+\cdots+E\left\|\mathbf{u}_{n}\right\|\right)^{20 d}
\end{aligned}
$$

pour $C_{0}$ assez grand. C'est cette inégalité qui requiert un majorant de $\operatorname{deg} \pi(\widetilde{\mathbf{G}})$ indépendant de $h(W)$, sans quoi l'inégalité (25) réintroduirait un terme en

$$
T \times \log (\text { d'une fonction de } h(W)) .
$$

La dernière inégalité résulte directement du corollaire I.9.5. Pour la première, on écrit

$$
\begin{aligned}
1+\left|w_{1}\right|+\cdots+\left|w_{d-1}\right| & \leq 1+d^{1 / 2}|\mathbf{w}| \\
& \leq 1+d^{1 / 2}\|\mathbf{w}\| \\
& \leq 1+d^{1 / 2}(\|\mathbf{u}\|+|\Lambda|) \\
& \leq 1+d^{1 / 2}\left(\|\mathbf{u}\|+\frac{1}{2} \mathrm{~d}\left(\mathbf{u}, t_{\widetilde{\mathbf{G}}}(\mathbb{C})\right)\right)
\end{aligned}
$$

et on utilise la minoration $\left|w_{d}\right| \geq \frac{1}{2(d+1)} \mathrm{d}\left(\mathbf{u}, t_{\widetilde{\mathbf{G}}}(\mathbb{C})\right)$ (proposition I.9.7).

\section{$\S$ 11. Rang du système linéaire}

Soit $P$ un polynôme multihomogène de $\mathbb{P}\left(=\mathbb{P}^{N_{0}} \times \cdots \times \mathbb{P}^{N_{n}}\right)$, à coefficients dans $\mathbf{L}$, de multidegré $\underline{D}:=\left(D_{0}, \ldots, D_{n}\right)$ (les entiers $D_{i}$ sont ceux définis au $\S$ I.7) et qui ne s'annule pas identiquement sur G. L'application $\Phi=\left(\Phi_{0}, \ldots, \Phi_{n}\right)$, définie page 17, est une application homolorphe de $t_{\mathbf{G}}(\mathbb{C})$ dans $\mathbb{C}^{\sum_{i=0}^{n}\left(N_{i}+1\right)} ;$ posons

$$
F=P \circ \Phi .
$$


Nous nous intéressons au rang du système linéaire - où les coordonnées des coefficients de $P$ dans la base de $\mathbf{L}$ sont, ici, vus comme des inconnues - défini par les conditions d'annulation suivantes :

$$
\forall(\underline{t}, s) \in \Upsilon, \quad \mathcal{D}_{\overline{\mathbf{f}}}^{\underline{t}} F(s \mathbf{u})=0 .
$$

Soit $E_{\mathbb{C}}:=(\mathbb{C}[\mathbb{P}] / \mathcal{I}(\mathbf{G}))_{D}$ l'ensemble des polynômes $P$, comme ci-dessus, à coefficients complexes $(\mathcal{I}(\mathbf{G})$ est l'idéal annulateur de $\mathbf{G}(\mathbb{C})$ dans $\mathbb{C}[\mathbb{P}])$. L'ensemble des classes des monômes $\mathrm{X}^{\lambda}$ de $\mathbb{C}[\mathbb{P}]_{\underline{D}}$ forme une famille génératrice de $E_{\mathbb{C}}$. Soit $\mathcal{B}$ une famille de tels monômes dont les classes forment une base de $E_{\mathbb{C}}$. Le polynôme $P$ peut s'écrire

$$
\sum_{\underline{\lambda}, i} a_{\underline{\lambda}, i} \cdot \xi_{i} \cdot \mathbf{X}^{\underline{\lambda}}
$$

où $a_{\underline{\lambda}, i} \in \mathbb{Q}$ et $\mathbf{X}^{\underline{\lambda}} \in \mathcal{B}$. Les inconnues du système (27) seront les $a_{\underline{\lambda}, i}$, qui sont au nombre de $\nu:=D \operatorname{dim} E_{\mathbb{C}}$.

Lemme I.11.1. Soit $\rho$ le rang du système (27). Il existe une constante $c_{17}$, ne dépendant que de $(\mathbf{G}, \Phi)$, telle que

$$
\rho \leq \frac{c_{17}}{C_{0}} \cdot \mathscr{H}\left(\mathbf{G} ; D_{0}^{\prime}, \ldots, D_{n}^{\prime}\right) \text { et } \nu \geq c_{17}^{-1} D \mathscr{H}\left(\mathbf{G} ; D_{0}^{\prime}, \ldots, D_{n}^{\prime}\right)
$$

où $D_{i}^{\prime}:=\max \left\{1, D_{i}\right\}$.

Démonstration. C'est le lemme 4.20 de [45], et aussi la scolie 6.13 de [65]. Le choix de $S_{0}$ (resp. $T_{0}$ ) joue un rôle important dans cette preuve pour le cas non-périodique ( resp. périodique).

Corollaire I.11.2. Les entiers $D_{i}$ sont tous non nuls, i.e. $D_{i}^{\prime}=D_{i}$.

Démonstration. Supposons que pour un indice $i \in\{0, \ldots, n\}$, l'entier $D_{i}$ est nul. D'après le lemme I.11.1, le nombre d'inconnues $\nu$ du système (27) est strictement supérieur au rang de ce système. Donc il existe un polynôme $P$, non identiquement nul sur $\mathbf{G}$, multihomogène de multidegré $\underline{D}$, solution de (27). En particulier $P$ s'annule à l'ordre $T_{0}$ en les points $s \mathbf{u}$

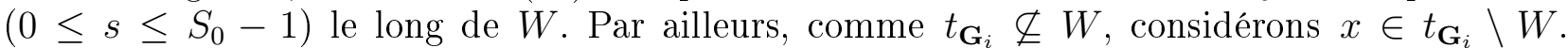
L'indépendance de la fonction $F$ vis-à-vis des variables relatives à $t_{\mathbf{G}_{i}}$ (c'est l'hypothèse $D_{i}=0$ ) implique que $F$ s'annule à un ordre infini le long de la droite engendrée par $x$. Ainsi $F$ s'annule à l'ordre $T_{0}$ en les points $s \mathbf{u}\left(0 \leq s \leq S_{0}-1\right)$ le long de $(W \otimes \mathbb{C}) \oplus \mathbb{C} \cdot x=t_{\mathbf{G}}(\mathbb{C})$. D'après le lemme de zéros de PhiLIPPON [63], il existe un sous-groupe connexe et propre $\mathbf{G}^{\prime}$ de $\mathbf{G}$ tel que

$$
T_{0}^{r^{\prime}} \times \mathscr{H}\left(\mathbf{G}^{\prime} ; D_{0}^{\prime}, \ldots, D_{n}^{\prime}\right) \times \operatorname{card}\left(\frac{\Gamma_{\mathbf{p}}\left(\left[\frac{S_{0}-1}{d+1}\right]\right)+\mathbf{G}^{\prime}(\mathbf{L})}{\mathbf{G}^{\prime}(\mathbf{L})}\right) \leq c_{18} \mathscr{H}\left(\mathbf{G} ; D_{0}^{\prime}, \ldots, D_{n}^{\prime}\right)
$$

Dans le cas général, nous en déduisons

$$
T_{0} \times \mathscr{H}\left(\mathbf{G}^{\prime} ; D_{0}^{\prime}, \ldots, D_{n}^{\prime}\right) \leq c_{18} \mathscr{H}\left(\mathbf{G} ; D_{0}^{\prime}, \ldots, D_{n}^{\prime}\right),
$$

(l'entier $r^{\prime}=\operatorname{codim}_{\mathbf{G}} \mathbf{G}^{\prime}$ est non nul car $\mathbf{G}^{\prime} \subsetneq \mathbf{G}$ ) ce qui contredit l'inégalité

$$
T_{0} \geq C_{0} \max \left\{1, D_{0}, \ldots, D_{n}\right\}
$$


Dans le cas particulier où $u_{0} \neq 0$ et $\prod_{j=1}^{n} \mathbf{G}_{j}$ est une variété semi-abélienne, nous pouvons être plus précis. En effet, dans ce cas, le sous-groupe $\mathbf{G}^{\prime}$ s'écrit $\mathbf{G}_{0}^{\prime} \times \mathbf{G}^{\prime \prime}$ où $\mathbf{G}_{0}^{\prime}=\{0\}$ ou $\mathbb{G}_{\mathrm{a}}$, et où $\mathbf{G}^{\prime \prime}$ est un sous-groupe algébrique (connexe) de $\prod_{j=1}^{n} \mathbf{G}_{j}$. Si $\mathbf{G}_{0}^{\prime}=\{0\}$ alors, comme $u_{0} \neq 0$, on a nécessairement

$$
\operatorname{card}\left(\frac{\Gamma_{\mathbf{p}}\left(\left[\frac{S_{0}-1}{d+1}\right]\right)+\mathbf{G}^{\prime}(\mathbf{L})}{\mathbf{G}^{\prime}(\mathbf{L})}\right)=\left[\frac{S_{0}-1}{d+1}\right]+1
$$

et $\mathscr{H}\left(\mathbf{G}^{\prime} ; D_{0}^{\prime}, \ldots, D_{n}^{\prime}\right)=\mathscr{H}\left(\mathbf{G}^{\prime \prime} ; D_{1}^{\prime}, \ldots, D_{n}^{\prime}\right)$. L'inégalité (28) devient alors

$$
T_{0}^{r^{\prime}} \times\left(\frac{S_{0}-1}{d+1}\right) \leq c_{19} D_{0}^{\prime} \times \max \left\{D_{1}^{\prime}, \ldots, D_{n}^{\prime}\right\}^{r^{\prime}-1}
$$

ce qui contredit

$$
T_{0} \geq C_{0} \max \left\{1, \frac{D_{0}}{S_{0}}, D_{1}, \ldots, D_{n}\right\}
$$

pour $C_{0}$ assez grand.

Si $\mathbf{G}_{0}^{\prime}=\mathbb{G}_{\mathrm{a}}$ alors

$$
\mathscr{H}\left(\mathbf{G}^{\prime} ; D_{0}^{\prime}, \ldots, D_{n}^{\prime}\right)=D_{0}^{\prime} \times \mathscr{H}\left(\mathbf{G}^{\prime \prime} ; D_{1}^{\prime}, \ldots, D_{n}^{\prime}\right)
$$

et $\operatorname{dim} \mathbf{G}^{\prime \prime}=\operatorname{dim} \mathbf{G}^{\prime}-1=d-r^{\prime}$ donc l'inégalité (28) implique

$$
T_{0}^{r^{\prime}} \times \mathscr{H}\left(\mathbf{G}^{\prime \prime} ; D_{1}^{\prime}, \ldots, D_{n}^{\prime}\right) \leq c_{20} \mathscr{H}\left(\prod_{j=1}^{n} \mathbf{G}_{j} ; D_{1}^{\prime}, \ldots, D_{n}^{\prime}\right)
$$

ce qui contredit $T_{0} \geq C_{0} \cdot \max \left\{1, D_{1}, \ldots, D_{n}\right\}$.

Remarque : La non-nullité des entiers $D_{i}$ sera utilisée implicitement au début de la démonstration du lemme I.14.3 (page 37).

\section{$\S 12$. Construction de la fonction auxiliaire}

Nous utiliserons le « classique »:

Lemme I.12.1 (de Thue-Siegel). Soit $\left(u_{i, j}\right)_{\substack{1 \leq i \leq j \\ 1 \leq j \leq \mu}} \in \mathrm{M}_{\nu, \mu}(\mathbb{C})$, de rang $\leq \rho$. Soient $\delta, m, p$ des réels positifs vérifiant

$$
\left(2 \mu e^{\delta+m+p}+1\right)^{2 \rho} \leq e^{\nu \delta} \text { et } \max _{1 \leq j \leq \mu}\left\{\sum_{i=1}^{\nu}\left|u_{i, j}\right|\right\} \leq e^{m} .
$$

Alors, il existe $\left(a_{1}, \ldots, a_{\nu}\right) \in \mathbb{Z}^{\nu} \backslash\{0\}$ tel que

$$
\max _{1 \leq i \leq \nu}\left\{\left|a_{i}\right|\right\} \leq e^{\delta} \quad \text { et } \max _{1 \leq j \leq \mu}\left\{\left|\sum_{i=1}^{\nu} u_{i, j} a_{i}\right|\right\} \leq e^{-p} .
$$


La démonstration de ce résultat est effectuée dans [65] (pages $301-303$ ). Les notations $\nu$ et $\mu$ (a priori « muettes ») ont été choisies de façon cohérente avec celles des paragraphes I.7 et I.11.

Le lemme technique qui suit - majoration d'un coefficient de Taylor - est une conséquence des formules de Cauchy. Le terme « remarquable» du majorant de ce coefficient de Taylor est $\varsigma_{0}^{\min \left(D_{0},|\underline{t}|\right)}$; l'exposant $\min \left(D_{0},|\underline{t}|\right)$ provient simplement du fait que la dérivée $|\underline{t}|^{\text {ème }}$ d'un monôme de degré $D_{0}$ est nulle si $|\underline{t}|>D_{0}$.

Lemme 1.12.2. Soient $H$ un nombre réel $\geq e, D_{0}, \ldots, D_{n} \in \mathbb{N}$ et $\ell \in \mathbb{N}^{*}$. Soit $\mathbf{x}:=$ $\left(\mathbf{x}_{i}\right)_{i \in\{1, \ldots, \ell\}} \in\left(t_{\mathbf{G}}(\mathbb{C})\right)^{\ell} ;$ les coordonnées de $\mathbf{x}_{i}$ sont $\left(x_{i, j}\right)_{j \in\{0, \ldots, d\}}$ dans la base e de $t_{\mathbf{G}}(\mathbb{C})$. Soient $\underline{t} \in \mathbb{N}^{\ell}$ et $\mathbf{z}=z_{0} e_{0}+\cdots+z_{d} e_{d} \in t_{\mathbf{G}}(\mathbb{C})$. Soit $P$ un polynôme multihomogène de multidegré $\left(D_{0}, \ldots, D_{n}\right)$ dont la somme des modules des coefficients est $\leq H$. Rappelons que $F$ désigne la fonction $P \circ \Phi$. Considérons $\varsigma_{0}, \varsigma_{1}$ vérifiant:

$$
\varsigma_{0} \geq \max \left\{1,\left|x_{1,0}\right|, \ldots,\left|x_{\ell, 0}\right|\right\} \quad \text { et } \varsigma_{1} \geq \max _{\substack{1 \leq i \leq \ell \\ 1 \leq j \leq d}}\left\{1,\left|x_{i, j}\right|\right\} \text {. }
$$

Il existe une constante $c_{21}$, ne dépendant que de $(\mathbf{G}, \Phi)$, telle que :

$$
\begin{aligned}
|\underline{\underline{t}} \underline{\underline{t}} \mathcal{D} \mathbf{\underline { x }} F(\mathbf{z})| \leq & \varsigma_{0}^{\min \left\{D_{0},|\underline{t}|\right\}} \times \varsigma_{1}^{|\underline{t}|} \times(\ell(d+1))^{|\underline{\underline{a}}|} \times H \times\left(1+\left|z_{0}\right|\right)^{D_{0}} \\
& \times \exp \left\{c_{21} \sum_{j=1}^{n} D_{j}\left(1+\left\|p_{j}^{\prime}(\mathbf{z})\right\|\right)^{\rho_{j}}\right\}
\end{aligned}
$$

où $p_{j}^{\prime}$ désigne la projection $t_{\mathbf{G}}(\mathbb{C}) \rightarrow t_{\mathbf{G}_{j}}(\mathbb{C})$.

Démonstration. On développe :

$$
\begin{aligned}
& \underline{\underline{t} !} \mathcal{D}_{\mathbf{x}}^{\underline{t}} F(\mathbf{z}) \\
& =\prod_{i=1}^{\ell} \frac{\left(\sum_{j=0}^{d} x_{i, j} \frac{\partial}{\partial z_{j}}\right)^{t_{i}}}{t_{i} !} F(\mathbf{z}) \\
& =\sum_{t_{i, 0}+\cdots+t_{i, d}=t_{i}}\left(\prod_{i \leq i \leq \ell} \frac{\left(x_{i, j}^{t_{i, j}}\right)}{\left(t_{i, j}\right) !}\right) \prod_{h=0}^{d}\left(\frac{\partial}{\partial z_{h}}\right)^{t_{1, h}+\cdots+t_{\ell, h}} F(\mathbf{z}) \\
& =\sum_{t_{i, 0}+\cdots+t_{i, d}=t_{i}}\left\{\left(\prod_{i, j} x_{i, j}^{t_{i, j}}\right) \times\left(\prod_{h=0}^{d} \frac{\left(t_{1, h}+\cdots+t_{\ell, h}\right) !}{t_{1, h} ! \cdots t_{\ell, h} !}\right) \times \prod_{h=0}^{d} \frac{\left(\frac{\partial}{\partial z_{h}}\right)^{t_{1, h}+\cdots+t_{\ell, h}}}{\left(t_{1, h}+\cdots+t_{\ell, h}\right) !} F(\mathbf{z})\right\}
\end{aligned}
$$

Dans le membre de droite ci-dessus, nous pouvons restreindre la somme à $t_{1,0}+\cdots+t_{\ell, 0} \leq D_{0}$ car $F$ est polynomiale en $z_{0}$ de degré au plus $D_{0}$. Nous en déduisons la majoration :

$$
\left|\frac{1}{\underline{t} !} \mathcal{D} \frac{\underline{t}}{\mathbf{x}} F(\mathbf{z})\right| \leq \varsigma_{0}^{\min \left(D_{0},|\underline{t}|\right)} \times \varsigma_{1}^{\mid \underline{|t|}} \times\left(\sum_{\substack{t_{i, 0}+\cdots+t_{i, d}=t_{i} \\ 1 \leq i \leq \ell}} \prod_{h=0}^{d} \frac{\left(t_{1, h}+\cdots+t_{\ell, h}\right) !}{t_{1, h} ! \cdots t_{\ell, h} !}\right) \times \max _{|\underline{\tau}|=|\underline{t}|}\left\{\left|\frac{1}{\tau !} \mathcal{D} \frac{\tau}{\mathbf{\tau}} F(\mathbf{z})\right|\right\}
$$


Comme $\prod_{h=0}^{d}\left(t_{1, h}+\cdots+t_{\ell, h}\right) ! \leq\left(\sum_{i, j} t_{i, j}\right) !=(|\underline{t}|) !$, on a

$$
\sum_{\substack{t_{i, 0}+\cdots+t_{i, d}=t_{i} \\ 1 \leq i \leq \ell}} \prod_{h=0}^{d} \frac{\left(t_{1, h}+\cdots+t_{\ell, h}\right) !}{t_{1, h} ! \cdots t_{\ell, h} !} \leq\{\ell(d+1)\}^{|\underline{\underline{t}}|}
$$

et donc

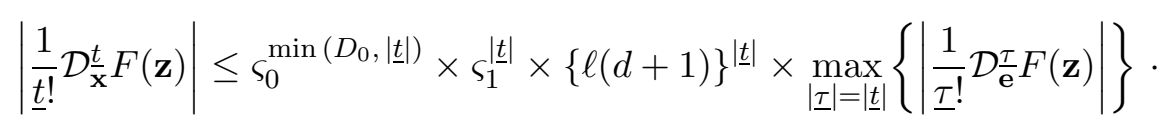

D'après l'inégalité de Cauchy, si nous posons

$$
\mathbf{z}_{\theta}:=e^{i \theta_{0}} e_{0}+\cdots+e^{i \theta_{d}} e_{d},
$$

on a

$$
\begin{aligned}
\left|\frac{1}{\underline{\tau} !} \mathcal{D} \frac{\tau}{\mathbf{e}} F(\mathbf{z})\right| & \leq \sup _{\theta_{j} \in[0,2 \pi]}\left\{\left|F\left(\mathbf{z}+\mathbf{z}_{\theta}\right)\right|\right\} \\
& \leq H \times\left(1+\left|z_{0}\right|\right)^{D_{0}} \times \sup _{\theta \in[0,2 \pi]^{d+1}}\left\{\prod_{j=1}^{n} \exp \left\{c_{j}^{+} D_{j}\left(1+\left\|p_{j}^{\prime}\left(\mathbf{z}+\mathbf{z}_{\theta}\right)\right\|_{j}\right)^{\rho_{j}}\right\}\right\}
\end{aligned}
$$

(cf. inégalité (10), page 15).

Alors, comme $1+\left\|p_{j}\left(\mathbf{z}+\mathbf{z}_{\theta}\right)\right\|_{j} \leq 1+\| p_{j}\left(\mathbf{z} \|_{j}+\sqrt{\delta_{j}}\right.$, nous obtenons

$$
\left|\frac{1}{\underline{\tau} !} \mathcal{D} \frac{\tau}{\mathbf{e}} F(\mathbf{z})\right| \leq H \times\left(1+\left|z_{0}\right|\right)^{D_{0}} \times \prod_{j=1}^{n} \exp \left\{c_{j}^{+} D_{j}\left(1+\left\|p_{j}(\mathbf{z})\right\|_{j}+\sqrt{\delta_{j}}\right)^{\rho_{j}}\right\}
$$

Ce qui, via l'inégalité (30), donne le résultat voulu.

Remarque : au vu de la démonstration, il apparaît clairement que l'on peut améliorer substantiellement cette majoration en considérant le vecteur $r_{0} e^{i \theta_{0}} w_{0}+\cdots+r_{d} e^{i \theta_{d}} w_{d}$ au lieu de $\mathbf{z}_{\theta}=e^{i \theta_{0}} w_{0}+\cdots+e^{i \theta_{d}} w_{d}$ et en ajustant $\left(r_{0}, \ldots, r_{d}\right)$ pour minimiser le membre de droite de (31). De cette manière, il est possible de faire apparaître (dans le majorant de $\left.\frac{1}{\underline{t} !}\left|\mathcal{D} \frac{t}{\mathbf{x}} F(\mathbf{z})\right|\right)$ un facteur de la forme

$$
\min \left(1, \frac{D_{0}+\cdots+D_{n}}{|\underline{t}|}\right)^{\mid \underline{|t|} / 2} .
$$

Mais, outre les complications inhérentes à cette optimisation, il est décevant de constater que, dans le cadre de notre étude, ce raffinement est inexploitable et dénué d'intérêt (sauf si on cherche à optimiser la constante dépendant de G), car le terme amélioré sera «négligeable » devant d'autres termes.

Proposition I.12.3. Pour $C_{0}$ assez grand, il existe un polynôme multihomogène $P$ de $\mathbf{L}[\mathbb{P}]$, de multidegré $\left(D_{0}, \ldots, D_{n}\right)$, ne s'annulant pas identiquement sur $\mathbf{G}$, tel que :

- Les coefficients de $P$ appartiennent à $\mathbb{Z} . \xi_{1} \oplus \cdots \oplus \mathbb{Z} . \xi_{D}$

- La hauteur $h(P)$ du polynôme $P$ est majorée par:

$$
C_{0}^{1 / 2} \cdot \frac{U_{0}}{D}
$$




\section{Le cas général}

- En posant $F=P \circ \Phi$ comme précédemment :

$$
\max _{(\underline{t}, s) \in \Upsilon}\left\{\left|\frac{1}{\underline{t} !} \mathcal{D}_{\mathbf{f}}^{\underline{t}} F(s \mathbf{u})\right|\right\} \leq e^{-C_{0} U_{0}}
$$

Démonstration. On écrit, a priori, $P=\sum_{\underline{\lambda}, i} a_{\underline{\lambda}, i} \cdot \xi_{i} . \mathbf{X}^{\underline{\lambda}}$ où $a_{\underline{\lambda}, i} \in \mathbb{Z}$ et $\mathbf{X}^{\underline{\lambda}} \in \mathcal{B}(c f . \S$ I.11). Pour $(\underline{t}, s) \in \Upsilon$, posons

$$
u_{(\underline{\lambda}, i)(\underline{t}, s)}=\xi_{i} \cdot \frac{1}{\underline{t} !} \cdot \mathcal{D}_{\underline{\mathbf{f}}}^{\underline{\mathbf{f}}}\left(\Phi^{\underline{\lambda}}\right)(s \mathbf{u}) .
$$

On notera $p_{\underline{\lambda}}:=\sum_{i} a_{\underline{\lambda}, i} \xi_{i}$ et $\mu$ le cardinal de $\Upsilon$. La matrice

$$
\left(u_{(\underline{\lambda}, i)(\underline{t}, s)}\right)_{\substack{\underline{\lambda}, i) \\(\underline{t})}} \in \mathrm{M}_{\nu, \mu}(\mathbb{C})
$$

est de rang $\rho$ (par définition de $\rho$ (lemme I.11.1)). Considérons

$$
\varsigma_{0}=(d+1)^{1 / 2} \max \left\{1,\left|\beta_{1}\right|, \ldots,\left|\beta_{d}\right|\right\}=(d+1)^{1 / 2}
$$

$\left(\right.$ car $\left|\beta_{i}\right| \leq 1$, voir page 17$)$ et

$$
\varsigma_{1}= \begin{cases}1 & \text { dans le cas non-périodique } \\ 1+\|\mathbf{u}\| & \text { dans le cas périodique }\end{cases}
$$

Appliquons le lemme I.12.2 au monôme $\mathbf{X}^{\underline{\lambda}}$, à la base $\mathbf{x}:=\mathbf{f}$, et au vecteur $\mathbf{z}:=s \mathbf{u}$ :

$$
\begin{aligned}
\left|\frac{1}{\underline{t} !} \mathcal{D}_{\underline{\mathbf{f}}}^{\underline{t}}\left(\Phi^{\underline{\lambda}}\right)(s \mathbf{u})\right| \leq & (d+1)^{\min \left\{D_{0},|\underline{t}|\right\} / 2} \times \varsigma_{1}^{|\underline{t}|} \times\{(d+1)(d+2)\}^{|\underline{t}|} \\
& \times\left(1+s\left\|\mathbf{u}_{0}\right\|\right)^{D_{0}} \times \exp \left\{c_{22} \sum_{j=1}^{n} D_{j}\left(1+s\left\|\mathbf{u}_{j}\right\|\right)^{\rho_{j}}\right\} \\
\leq & c_{23}^{T} \times\left(1+S\left\|\mathbf{u}_{0}\right\|\right)^{D_{0}} \times e^{c_{23} \sum_{j=1}^{n} D_{j}\left(1+S\left\|\mathbf{u}_{j}\right\|\right)^{\rho_{j}}} \\
\leq & e^{c_{24} U_{0}}
\end{aligned}
$$

Ainsi, il existe une constante $c_{25}$, ne dépendant que de $(\mathbf{G}, \Phi)$, telle que :

$$
\begin{aligned}
\max _{(\underline{t}, s) \in \Upsilon}\left\{\sum_{\underline{\lambda}}\left|\frac{1}{\underline{t} !} \mathcal{D}^{\underline{\underline{f}}}\left(\Phi^{\underline{\lambda}}\right)(s \mathbf{u})\right|\right\} & \leq \mathscr{H}\left(\mathbf{G} ; D_{0}, \ldots, D_{n}\right) \times e^{c_{25} U_{0}} \\
& \leq e^{2 c_{25} U_{0}}
\end{aligned}
$$

car $\mathscr{H}\left(\mathbf{G} ; D_{0}, \ldots, D_{n}\right) \leq\left(\operatorname{deg}_{\Phi} \mathbf{G}\right) U_{0}^{n+1} \leq e^{c_{25} U_{0}}$, pour $C_{0}$ assez grand. Comme

$$
\begin{aligned}
\sum_{i=1}^{D}\left|\xi_{i}\right| & \leq D \max \left\{\left|\xi_{1}\right|, \ldots,\left|\xi_{D}\right|\right\} \\
& \leq D \times e^{D h\left(\xi_{1}: \cdots: \xi_{D}\right)} \\
& \leq e^{U_{0}}
\end{aligned}
$$


nous en déduisons une majoration de :

$$
\begin{aligned}
\max _{(\underline{t}, s) \in \Upsilon}\left\{\sum_{(\underline{\lambda}, i)}\left|u_{(\underline{\lambda}, i),(\underline{t}, s)}\right|\right\} & \leq\left(\sum_{i=1}^{D}\left|\xi_{i}\right|\right) \times \max _{(\underline{\underline{t}, s) \in \Upsilon}}\left\{\sum_{\underline{\underline{\lambda}}}\left|\frac{1}{\underline{t} !} \mathcal{D}_{\underline{\mathbf{f}}}^{\underline{\underline{t}}}\left(\Phi^{\underline{\lambda}}\right)(s \mathbf{u})\right|\right\} \\
& \leq e^{c_{26} U_{0}} .
\end{aligned}
$$

Par ailleurs, $\nu \geq c_{27} D \mathscr{H}\left(\mathbf{G} ; D_{0}, \ldots, D_{n}\right)$ et

$$
\frac{1}{\rho} \geq \frac{C_{0}}{c_{28}} \cdot \frac{1}{\mathscr{H}\left(\mathbf{G} ; D_{0}, \ldots, D_{n}\right)}
$$

(lemme I.11.1 et corollaire I.11.2) donc

$$
\frac{\nu}{2 \rho} \geq \frac{C_{0} D}{c_{29}} .
$$

Pour que la condition (du lemme de Thue-Siegel)

$$
\left(2 \mu e^{\delta+m+p}+1\right)^{2 \rho} \leq e^{\nu \delta}
$$

soit vérifiée, nous choisissons $p:=C_{0} U_{0}, m:=c_{30} U_{0}$ et $\delta:=C_{0}^{1 / 4} \cdot \frac{U_{0}}{D}$ (voir également majoration de $\mu$ page 22). Ainsi, le lemme de Thue-Siegel permet de construire un tel polynôme $P$, de coefficients $p_{\underline{\lambda}}=\sum_{i} a_{\underline{\lambda}, i} \cdot \xi_{i}$, avec $a_{\underline{\lambda}, i} \in \mathbb{Z}$ non tous nuls et $\max _{\underline{\lambda}, i}\left\{\left|a_{\underline{\lambda}, i}\right|\right\} \leq e^{\delta}$. Par conséquent :

- Pour une place $\mathfrak{P}$ ultramétrique :

$$
\left|p_{\underline{\lambda}}\right|_{\mathfrak{P}} \leq \max _{1 \leq i \leq D}\left\{\left|\xi_{i}\right|_{\mathfrak{P}}\right\}
$$

- Pour une place $\sigma$ archimédienne :

$$
\begin{aligned}
\left|p_{\underline{\lambda}}\right|_{\sigma} & \leq D \cdot \max _{i}\left\{\left|a_{\underline{\lambda}, i}\right|\right\} \cdot \max _{1 \leq i \leq D}\left\{\left|\xi_{i}\right|_{\sigma}\right\} \\
& \leq\left(D e^{\delta}\right) \cdot \max _{1 \leq i \leq D}\left\{\left|\xi_{i}\right|_{\sigma}\right\}
\end{aligned}
$$

Donc

$$
h(P) \leq \log \left(D e^{\delta}\right)+h\left(\xi_{1}: \cdots: \xi_{D}\right) \leq \log D+C_{0}^{1 / 4} \cdot \frac{U_{0}}{D}+\frac{U_{0}}{D} \leq C_{0}^{1 / 2} \cdot \frac{U_{0}}{D}
$$

pour $C_{0}$ assez grand. Ce qui clôt la démonstration.

Tout le travail préparatoire (élimination des éventuels « mauvais » sous-groupes et construction du polynôme auxiliaire) étant effectué, le schéma de démonstration est le suivant: considérons $\frac{1}{t !} \mathcal{D} \underset{\widetilde{\mathbf{e}}}{\underline{t}} F(m \mathbf{u})$ le premier* coefficient de Taylor non nul. Un tel coefficient existe car, dans le cas contraire, le lemme de zéros de Philippon entrerait en conflit avec le choix des paramètres (voir lemme I.14.3, page 37). Ce terme est proportionnel à un élément $\Xi \in \mathbf{L}$.

1. Nous évaluons la norme $\mathfrak{P}$-adique de $\Xi(\S \mathrm{I} .14)$.

2. Nous évaluons la norme $\sigma$-archimédienne de $\Xi$, pour tout plongement $\sigma: \mathbf{L} \rightarrow \mathbb{C}(\S \mathrm{I} .15)$.

*Pour l'ordre lexicographique sur $\mathbb{N} \times \mathbb{N}^{d}$. 
3. La quantité $\frac{1}{j !} \mathcal{D}_{\overline{\mathbf{f}}}^{\frac{j}{F}} F(s \mathbf{u})$ est « petite $» \operatorname{pour}(\underline{j}, s) \in \Upsilon$ (et relativement à la valeur absolue associée au plongement initial $\sigma_{0}$ de $\mathbf{L}$ dans $\mathbb{C}$ ). Nous commençons alors un raisonnement par l'absurde et nous supposons que la distance de $\mathbf{u}$ à $W$ est non nulle et plus petite qu'une certaine quantité. Par extrapolation, nous montrons alors que le terme $\frac{1}{j !} \mathcal{D}_{\overline{\mathbf{f}}}^{j} F(s \mathbf{u})$ reste « petit » pour $|\underline{j}| \leq(d+1) T$ et $s \leq(d+1) S$. Nous en déduisons alors, par changement de base, une estimation de $\frac{1}{t !} \mathcal{D} \underset{\widetilde{\mathbf{e}}}{t} F(m \mathbf{u})$ puis de $\Xi$ (c'est l'objet du paragraphe suivant).

4. Nous aurons alors un nombre algébrique $\Xi$ «petit » pour toutes les valeurs absolues associées aux différentes places de $\mathbf{L}$. La formule $d u$ produit impliquera la nullité de ce nombre. Ce qui est en contradiction avec la définition du couple $(\underline{t}, m)$. Nous en déduirons alors la minoration idoine de $|\Lambda|$.

\section{$\S$ 13. Extrapolation}

Dorénavant, nous raisonnerons par l'absurde et nous supposerons que

$$
|\Lambda| \leq e^{-C_{0} U_{0}} .
$$

Voici un lemme préliminaire :

Lemme I.13.1. Soit F la fonction holomorphe associée au polynôme construit précédemment. Il existe une constante $c_{31}>0$, ne dépendant que de $(\mathbf{G}, \Phi)$, telle que, pour tout couple $(\underline{t}, s) \in$ $\mathbb{N}^{d+1}$ avec $|\underline{t}| \leq 2(d+1) T$ et $s \leq 2(d+1) S$, l'inégalité suivante soit vérifiée :

$$
\left|\frac{1}{t !} \mathcal{D}_{\mathbf{f}}^{\underline{t}} F(s \mathbf{u})-\frac{1}{\underline{t} !} \mathcal{D}_{\mathbf{f}}^{\underline{t}} F(s \mathbf{w})\right| \leq e^{-c_{31} C_{0} U_{0}}
$$

Démonstration. C'est une conséquence de l'inégalité des accroissements finis. Considérons, pour un couple $(\underline{t}, s)$ et un réel $x$, la fonction

$$
f(x)=\frac{1}{\underline{t} !} \mathcal{D}_{\mathbf{f}}^{\underline{t}} F(s \mathbf{u}+x s(\mathbf{w}-\mathbf{u})) .
$$

Cette fonction est dérivable sur $[0,1]$ et donc $|f(0)-f(1)| \leq \max _{x \in[0,1]}\left|f^{\prime}(x)\right|$. Or

$$
f^{\prime}(x)=\sum_{i=0}^{d} s\left(w_{i}-u_{i}\right) \frac{\partial}{\partial z_{i}}\left(\frac{1}{t !} \mathcal{D} \frac{\underline{t}}{\mathbf{f}} F(s \mathbf{u}+x s(\mathbf{w}-\mathbf{u}))\right)
$$

donc

$$
\left|f^{\prime}(x)\right|=s|\Lambda|\left|\frac{\partial}{\partial z_{0}}\left(\frac{1}{\underline{t} !} \mathcal{D}_{\mathbf{f}}^{\underline{t}} F(s \mathbf{u}+x s(\mathbf{w}-\mathbf{u}))\right)\right| .
$$

La majoration (34) découle du lemme I.12.2 appliqué à $\ell:=d+1, \mathbf{x}:=\left(e_{0}, \mathbf{f}\right), \underline{t}^{\prime}:=(1, \underline{t})$, $\mathbf{z}:=s \mathbf{u}+x s(\mathbf{w}-\mathbf{u}), H:=e^{C_{0}^{1 / 2} U_{0}}, \varsigma_{0}:=(d+1)^{1 / 2}$ et $\varsigma_{1}:=1+\|\mathbf{u}\|$ (on a pris 1 dans le cas non-périodique), et de l'inégalité $2(d+1) S|\Lambda| \leq 1$ (conséquence de l'hypothèse (33)). 
Remarque : Dans la démonstration de ce lemme, au lieu d'invoquer le lemme I.12.2 pour $\left(e_{0}, \mathbf{f}\right)$, nous aurions pu écrire les vecteurs de la base $\mathbf{f}$ dans une base orthonormée $\mathbf{f}^{\prime}$ de $t_{\mathbf{G}}(\mathbb{C})$ (base indépendante de $W$, c'est important). Les coefficients de la matrice de passage entre ces deux bases orthonormées seraient $\leq 1$. Ensuite on aurait utilisé le lemme I.10.1 (de changement de bases) puis le lemme I.12.2 pour $\left(e_{0}, \mathbf{f}^{\prime}\right)$. Cela aurait éviter à l'astuce $\varsigma_{0}^{\min }\left\{D_{0},|\underline{\mid l}|\right\}$ d'intervenir ici.

La proposition suivante est le cœur de l'extrapolation (analytique) que nous voulons effectuer. Sa preuve repose sur un lemme de Schwarz approché.

Proposition I.13.2. Pour $C_{0}$ assez grand, pour tout réel $E \geq 1$, pour tout $(\underline{t}, s) \in \mathbb{N}^{d+1}$ avec $|\underline{t}| \leq(d+1) T$ et $s \leq(d+1) S$, on a l'inégalité

$$
\left|\frac{1}{\underline{t} !} \mathcal{D}_{\mathbf{f}}^{\underline{t}} F(s \mathbf{u})\right| \leq \exp \left\{-C_{0}^{3 / 4} U_{0}\right\}
$$

Démonstration. Par construction de $P$, pour tout $(\underline{t}, s) \in \Upsilon$, on a

$$
\begin{aligned}
\left|\frac{1}{\underline{t} !} \mathcal{D}_{\mathbf{f}}^{\underline{t}} F(s \mathbf{w})\right| & \leq\left|\frac{1}{\underline{t} !} \mathcal{D}_{\mathbf{f}}^{\underline{t}} F(s \mathbf{u})\right|+\left|\frac{1}{\underline{t} !} \mathcal{D}_{\frac{\mathbf{f}}{t}} F(s \mathbf{u})-\frac{1}{\underline{t} !} \mathcal{D}_{\mathbf{f}}^{\underline{t}} F(s \mathbf{w})\right| \\
& \leq e^{-c_{32} C_{0} U_{0}}
\end{aligned}
$$

pour une certaine constante $c_{32}$, ne dépendant que de $(\mathbf{G}, \Phi)$.

- Dans le cas non-périodique, fixons un $d$-uplet $\underline{t}=\left(t_{1}, \ldots, t_{d}\right)$ tel que $t_{1}+\cdots+t_{d} \leq(d+1) T$ et posons

$$
f(z)=\frac{1}{\underline{t} !} \mathcal{D}_{\mathbf{f}}^{\underline{t}} F(z \mathbf{w}) .
$$

- Dans le cas périodique, nous fixons également $\underline{t}=\left(t_{1}, \ldots, t_{d-1}, t_{d}\right)$ avec $|\underline{t}| \leq(d+1) T$ mais nous posons

$$
f(z)=\frac{1}{t_{1} ! \cdots t_{d-1} !} \mathcal{D}_{\mathbf{f}}^{\left(t_{1}, \ldots, t_{d-1}, 0\right)} F(z \mathbf{w}) .
$$

La fonction complexe $f$ ainsi définie est analytique, et les dérivées sont

$$
\frac{1}{m !} f^{(m)}(z)=\sum_{\substack{j \in \mathbb{N}^{d} \\
j_{1}+\cdots+j_{d}=m}}\left(\begin{array}{c}
\underline{t}+\underline{j} \\
\underline{j}
\end{array}\right) \mathfrak{w}_{1}^{j_{1}} \cdots \mathfrak{w}_{d}^{j_{d}} \frac{1}{(\underline{t}+\underline{j}) !} \mathcal{D}_{\mathbf{f}}^{\underline{t+j}}-\underline{j}(z \mathbf{w})
$$

où $\mathfrak{w}_{1}, \ldots, \mathfrak{w}_{d}$ sont les composantes de w dans la base $\mathbf{f}$. Le « lemme de Schwarz approché », évoqué en préambule de la proposition, qui est au centre du processus d'extrapolation est le suivant :

Lemme I.13.3 ( [81]). Soient $f$ une fonction analytique dans le disque de centre 0 et de rayon $R \geq 4, S_{1}$ un entier $\geq 2, r \in\left[S_{1}, R / 2\right]$ et $T_{1}$ un entier naturel. Alors

$$
|f|_{2 r} \leq 2|f|_{R}\left(\frac{4 r}{R}\right)^{T_{1} S_{1}}+5\left(\frac{18 r}{S_{1}}\right)^{T_{1} S_{1}} \times \max _{\substack{0 \leq m<T_{1} \\ 0 \leq h<S_{1}}}\left\{\left|\frac{1}{m !} f^{(m)}(h)\right|\right\} .
$$


(1) Cas non-périodique

Nous allons appliquer le lemme précédent, pour extrapoler sur les points, avec $r:=(d+$ 1) $S / 2, R:=2(d+1) S E, T_{1}:=(d+1) T$ et $S_{1}:=S_{0}$. L'égalité (37) permet de majorer

$$
\max \left\{\left|\frac{1}{m !} f^{(m)}(h)\right| ; 0 \leq m<(d+1) T \text { et } 0 \leq h<S_{0}\right\}
$$

par

$$
\left(d+\left|\mathfrak{w}_{1}\right|+\cdots+\left|\mathfrak{w}_{d}\right|\right)^{2(d+1) T} \times \max _{(\underline{h}, s) \in \Upsilon}\left|\frac{1}{h !} \mathcal{D}_{\underline{\mathbf{f}}}^{\underline{h}} F(s \mathbf{w})\right|
$$

puis, via l'inégalité (36), par $e^{-c_{33} C_{0} U_{0}}$. De plus, d'après la proposition I.10.2 (changement de bases de dérivation) et comme $2(d+1) S E|\Lambda| \leq 1$ (i.e. $R|\Lambda| \leq 1$, c'est encore une conséquence de l'hypothèse (33)), on a

$$
|f|_{R} \leq d^{3(d+1) T / 2} \times \max _{\substack{|z|=R \\|\underline{h}| \leq(d+1) T}}\left\{\left|\frac{1}{\underline{h} !} \mathcal{D} \underset{\widetilde{\mathbf{e}}}{\widetilde{h}} F(z \mathbf{w})\right|\right\} \leq e^{c_{34} U_{0}}
$$

Le lemme I.13.3 permet alors d'obtenir la majoration annoncée de $\left|\frac{1}{\underline{t} !} \mathcal{D}_{\mathbf{f}}^{\underline{t}} F(s \mathbf{u})\right|$.

(2) Cas périodique

Dans ce cas, par définition de la base $\mathbf{f}$, qui contient le vecteur $\mathbf{w}$, la formule de dérivation (37) se simplifie :

$$
\forall m \in \mathbb{N}, \quad \frac{1}{m !} f^{(m)}(z)=\frac{1}{t_{1} ! \cdots t_{d-1} ! m !} \mathcal{D}_{\mathbf{f}}^{\left(t_{1}, \ldots, t_{d-1}, m\right)} F(z \mathbf{w}) .
$$

Par conséquent, la construction de $P$ et le lemme I.13.1 impliquent que le terme

$$
\max _{\substack{0 \leq m \leq T_{0} \\ 0 \leq s<2(d+1) S}}\left\{\left|\frac{1}{m !} f^{(m)}(s)\right|\right\}
$$

est inférieur à $e^{-c_{35} C_{0} U_{0}}$. Nous appliquons le lemme d'interpolation avec $R:=4(d+1) S E$, $T_{1}:=T_{0}, r=S_{1}:=(d+1) S$. L'inégalité de Cauchy :

$$
\frac{1}{t_{d} !}\left|f^{\left(t_{d}\right)}(s)\right| \leq|f|_{(d+1) S+1} \leq|f|_{2(d+1) S}
$$

pour $s \in\{0, \ldots,(d+1) S\}$, et, de nouveau, le lemme I.13.3 permettent d'obtenir la majoration voulue.

Corollaire I.13.4. Pour $C_{0}$ assez grand, pour tous $(\underline{t}, s) \in \mathbb{N}^{d+1}$ avec $|\underline{t}| \leq(d+1) T$ et $s \leq(d+1) S$, la quantité $\left|\frac{1}{\underline{t} !} \mathcal{D} \underline{\underline{\mathbf{e}}} F(s \mathbf{u})\right|$ est majorée par $\exp \left\{-C_{0}^{5 / 8} U_{0}\right\}$.

Démonstration. Nous appliquons la proposition I.10.2 de changement de bases de dérivations. Dans le cas non-périodique, le corollaire est immédiat. Dans le cas périodique, avec la remarque qui suit la proposition I.10.2, nous avons

$$
\begin{aligned}
\log \left|\frac{1}{\underline{t} !} \mathcal{D} \underline{\underline{\mathbf{e}}} F(s \mathbf{u})\right| \leq & c_{36} T \log \left(C_{0}^{23 d}\left(D+D h\left(\mathbf{p}_{1}\right)+\cdots+D h\left(\mathbf{p}_{n}\right)+E\left\|\mathbf{u}_{1}\right\|+\cdots+E\left\|\mathbf{u}_{n}\right\|\right)^{20 d}\right) \\
& \quad+\max _{|\underline{t}| \leq(d+1) T} \log \left|\frac{1}{t !} \mathcal{D}_{\underline{\mathbf{f}}}^{\underline{t}} F(s \mathbf{u})\right| \\
\leq & C_{0}^{1 / 4} U_{0}-C_{0}^{3 / 4} U_{0} \leq-C_{0}^{5 / 8} U_{0} \quad \text { pour } C_{0} \quad \text { assez grand. }
\end{aligned}
$$


Ce corollaire conclut la partie analytique de la démonstration. Le paragraphe suivant a trait à la partie arithmétique qui est au centre de ce texte.

\section{$\S$ 14. Estimations ultramétriques}

Notons $M_{\mathbf{L}, f}$ l'ensemble des places finies de $\mathbf{L}$.

Définition I.14.1. Soient $n$ et $k$ des entiers naturels non nuls. Nous noterons $\delta_{k}(n)$ l'entier :

$$
\operatorname{ppcm}\left\{i_{1} \cdots i_{h} ; i_{j} \in \mathbb{N}^{*}, h \leq n, i_{1}+\cdots+i_{h} \leq k\right\}
$$

Dans [25], S. BRUiLTeT a démontré* le

Lemme I.14.2. Pour tout $n, k \in \mathbb{N}^{*}$, on a

$$
\log \delta_{k}(n) \leq k \log (4 n) .
$$

Lemme I.14.3. Parmi les nombres $\frac{1}{t !} \mathcal{D}_{\widetilde{\mathbf{e}}}^{t} F(m \mathbf{u})$, avec $0 \leq|\underline{t}| \leq(d+1) T$ et $0 \leq m \leq(d+1) S$, au moins un n'est pas nul.

Démonstration. Si tous les nombres complexes $\mathcal{D} \underset{\widetilde{\mathbf{e}}}{\underline{t}} F(m \mathbf{u})$ étaient nuls, alors (par définition) $P$ s'annulerait à l'ordre $(d+1) T$ le long de $W$ en tous les points $s \mathbf{u}, s \in\{0, \ldots,(d+1) S\}$ et, d'après le lemme de zéros de Philippon, il existerait un sous-groupe connexe et propre $\mathbf{G}^{\prime}$ de $\mathrm{G}$ vérifiant l'inégalité

$$
T^{\operatorname{codim}_{W}\left(W \cap t_{\mathbf{G}^{\prime}}\right)} \times \operatorname{card}\left(\frac{\Gamma_{\mathbf{p}}(S)+\mathbf{G}^{\prime}(\mathbf{L})}{\mathbf{G}^{\prime}(\mathbf{L})}\right) \times \mathscr{H}\left(\mathbf{G}^{\prime} ; D_{0}, \ldots, D_{n}\right) \leq c_{37} \mathscr{H}\left(\mathbf{G} ; D_{0}, \ldots, D_{n}\right) .
$$

La proposition I.7.1 exclut le cas où $t_{\mathbf{G}^{\prime}} \subseteq W$. Le même argument que celui donné à la fin de la preuve du corollaire I.11.2 élimine l'autre cas. Donc $\mathbf{G}^{\prime}$ ne peut pas exister et le lemme est démontré.

Fixons un couple $(m, \underline{t})$ tel que $\mathcal{D}_{\widetilde{\mathbf{e}}}^{t} F(m \mathbf{u}) \neq 0$ et $\mathcal{D}_{\widetilde{\mathbf{e}}}^{\underline{h}} F(m \mathbf{u})=0$ pour tout $d$-uplet $\underline{h}$ de longueur $<|\underline{t}|$. Soit $\left(\tau_{0}, \ldots, \tau_{n}\right) \in\left\{0, \ldots, N_{0}\right\} \times \cdots \times\left\{0, \ldots, N_{n}\right\}$ tel que $\left|\varphi_{\tau_{j}}^{(j)}\left(m \mathbf{u}_{j}\right)\right|$ soit non-nul pour $0 \leq j \leq n$. Si nous regardons $F$ comme une section du fibré $\mathcal{O}_{\mathbf{G}}\left(D_{0}, \ldots, D_{n}\right)$, le choix de $\left(\tau_{0}, \ldots, \tau_{n}\right)$ correspond au choix d'une trivialisation de cette section au voisinage de $m$ p.

Lemme I.14.4. Le nombre complexe

$$
\frac{1}{\prod_{j=0}^{n}\left(\varphi_{\tau_{j}}^{(j)}\left(m \mathbf{u}_{j}\right)\right)^{D_{j}}} \cdot \frac{1}{\underline{t} !} \mathcal{D}_{\widetilde{\mathbf{e}}}^{t} F(m \mathbf{u})
$$

est un élément de $\mathbf{L}$.

\footnotetext{
*On dispose aussi (ibid.) de l'estimation asymptotique $\frac{1}{k} \log \delta_{k}(n) \underset{k \rightarrow+\infty}{\longrightarrow} \sum_{i=1}^{n} \frac{1}{i}$.
} 
Démonstration. En vertu de la formule de Leibniz, le nombre complexe (39) est la dérivée (divisée, le long de $W$, à l'ordre $\underline{t}$, au point $m \mathbf{u}$ ) de la fonction

$$
\frac{F(\mathbf{z})}{\prod_{j=0}^{n}\left(\varphi_{\tau_{j}}^{(j)}(\mathbf{z})\right)^{D_{j}}}
$$

qui est un polynôme en $\left(\psi_{\tau_{j}}^{(j)}\right)_{j=0, \ldots, n}$ (voir définition de $\psi_{\tau_{j}}^{(j)}$, page 16). Les formules de dérivations liées aux groupes $\mathbf{G}_{j}$ (du type de celle (11), page 16) permettent alors d'en déduire ce lemme.

Le point clef de la démonstration des théorèmes I.3.1 et I.3.2 est le

Lemme I.14.5. Il existe un ensemble fini $\mathcal{S}$ de places de $\mathbf{L}$, dont la trace sur $\mathbf{K}$ (i.e. $\{\mathfrak{P} \cap$ $\left.\left.\mathcal{O}_{\mathbf{K}} ; \mathfrak{P} \in \mathcal{S}\right\}\right)$ ne dépend que de $(\mathbf{G}, \Phi)$, il existe des constantes $\left(\mathbf{c}_{j}\right)_{j \in\{0, \ldots, n\}} \quad\left(\mathbf{c}_{0}=1\right)$ ne dépendant que de $(\mathbf{G}, \Phi)$, tels que, pour toute place $\mathfrak{P} \notin \mathcal{S}$, l'inégalité suivante soit vérifiée :

$$
\begin{aligned}
& \left|\frac{1}{\prod_{j=0}^{n}\left(\varphi_{\tau_{j}}^{(j)}\left(m \mathbf{u}_{j}\right)\right)^{D_{j}}} \frac{1}{\underline{t} !} \mathcal{D}_{\widetilde{\mathbf{e}}}^{\underline{\underline{\mathbf{e}}}} F(m \mathbf{u})\right|_{\mathfrak{P}} \leq \max \left\{1,\left|\beta_{1}\right|_{\mathfrak{P}}, \ldots,\left|\beta_{d}\right|_{\mathfrak{P}}\right\}^{D_{0}} \times \max _{\underline{\underline{\lambda}}}\left\{\left|p_{\underline{\underline{\lambda}}}\right|_{\mathfrak{P}}\right\} \\
& \times \frac{1}{\left|\delta_{|\underline{t}|}\left(D_{0}\right)\right|_{\mathfrak{P}}} \times \prod_{h=0}^{n} \frac{1}{\left|A_{\tau_{h}}^{(h)}\left(\psi_{0}^{(h)}(0), \psi_{\tau_{h}}^{(h)}\left(m \mathbf{u}_{h}\right)\right)\right|_{\mathfrak{P}}^{D_{h}}} \\
& \times \prod_{j=0}^{n} \max \left\{\left|\frac{\varphi_{0}^{(j)}\left(m \mathbf{u}_{j}\right)}{\varphi_{\tau_{j}}^{(j)}\left(m \mathbf{u}_{j}\right)}\right|_{\mathfrak{P}}, \cdots,\left|\frac{\varphi_{N_{j}}^{(j)}\left(m \mathbf{u}_{j}\right)}{\varphi_{\tau_{j}}^{(j)}\left(m \mathbf{u}_{j}\right)}\right|_{\mathfrak{P}}\right\}^{\mathbf{c}_{j} D_{j}}
\end{aligned}
$$

où $|\cdot|_{\mathfrak{P}}$ est la valeur absolue sur $K_{\mathfrak{P}}$ définie $p$. ix.

Démonstration. Rappelons que $\mathcal{G} \rightarrow \operatorname{Spec} \mathcal{O}_{\mathbf{K}}[r]$ désigne le modèle lisse de $\mathbf{G}$, que nous avons considéré au $\S$ I.5. Nous noterons $A:=\mathcal{O}_{\mathbf{K}}[r]$ (anneau principal).

Nous allons tout d'abord utiliser les formules d'additions sur G (relatives au plongement $\Phi$ ) pour nous ramener à $m=0$. La nullité des dérivées d'ordre $<\underline{t}$ de $F$ au point $m \mathbf{u}$ implique*,

*Dans la littérature, cette première étape porte le nom d'《 astuce d' Anderson-BAKER-CoATES 》. Elle vise à dissocier la contribution des dérivations et celle du point $m \mathbf{p}$. Dans un formalisme géométrique plus adéquat ( $c f$. seconde partie), elle est implicite et n'apparaît plus comme une « astuce ». 
en utilisant la formule de Leibniz,

$$
\begin{aligned}
& \frac{1}{\prod_{j=0}^{n}\left(\varphi_{\tau_{j}}^{(j)}\left(m \mathbf{u}_{j}\right)\right)^{D_{j}}} \frac{1}{\underline{t} !} \mathcal{D} \underline{\underline{\underline{e}}} F(m \mathbf{u}) \\
& =\frac{1}{\underline{t} !} \mathcal{D} \underline{\underline{\tilde{\mathbf{e}}}}\left(P \circ\left(\psi_{\tau_{0}}^{(0)}, \ldots, \psi_{\tau_{n}}^{(n)}\right)(z+m \mathbf{u})\right)(0) \\
& =\frac{1}{\underline{t} !} \mathcal{D} \underline{\underline{\tilde{\mathbf{e}}}}\left(P \circ\left(\left(\frac{A_{0}^{(h)}}{A_{\tau_{h}}^{(h)}}\left(\varphi(z), \varphi\left(m \mathbf{u}_{h}\right)\right), \ldots, \frac{A_{N_{h}}^{(h)}}{A_{\tau_{h}}^{(h)}}\left(\varphi(z), \varphi\left(m \mathbf{u}_{h}\right)\right)\right)_{h}\right)\right)(0) \\
& =\prod_{h=0}^{n} \frac{1}{A_{\tau_{h}}^{(h)}\left(\left(\psi_{0}^{(0)}, \ldots, \psi_{0}^{(n)}\right),\left(\psi_{\tau_{0}}^{(0)}\left(m \mathbf{u}_{h}\right), \ldots, \psi_{\tau_{n}}^{(n)}\left(m \mathbf{u}_{h}\right)\right)\right)^{D_{h}}} \\
& \times \frac{1}{\underline{t} !} \mathcal{D}_{\widetilde{\mathbf{e}}}^{\underline{t}}\left(Q\left(\psi_{0}^{(0)}, \ldots, \psi_{0}^{(n)}\right)\right)(0)
\end{aligned}
$$

où, si nous posons $\mathbf{X}_{h}:=\left(X_{0}^{(h)}, \ldots, X_{N_{h}}^{(h)}\right)$,

$$
Q\left(\mathbf{X}_{0}, \ldots, \mathbf{X}_{n}\right)=P\left(\left(A_{0}^{(h)}\left(\mathbf{X}_{h}, \psi_{\tau_{h}}^{(h)}\left(m \mathbf{u}_{h}\right)\right), \ldots, A_{N_{h}}^{(h)}\left(\mathbf{X}_{h}, \psi_{\tau_{h}}^{(h)}\left(m \mathbf{u}_{h}\right)\right)\right)_{0 \leq h \leq n}\right) .
$$

Le polynôme $Q$ est homogène en $\mathbf{X}_{h}$ de degré $\mathbf{c}_{h} D_{h}$ où

$$
\mathbf{c}_{h}:=\operatorname{deg}_{\mathbf{X}} A_{0}^{(h)}(\mathbf{X}, \mathbf{Y})
$$

(cela a un sens par définition des polynômes $A_{0}^{(h)}$, page 14).

Soit $\mathcal{F}$ l'ensemble des coefficients de tous les polynômes $A_{j}^{(h)}$ et $C_{k j h}^{(i)}$ (ces polynômes, définis à la fin du $\S \mathrm{I} .5$, sont en nombre fini). C'est un sous-ensemble fini de K. Soit $\mathcal{S}$ l'ensemble des places ultramétriques $\mathfrak{P}$ de $\mathbf{L}$ qui divisent $r^{-1}$ ou telles qu'il existe un élément de $\mathcal{F}$ qui n'appartiennent pas à

$$
\mathcal{O}_{\mathbf{L}_{\mathfrak{P}}}=\left\{x \in \mathbf{L}_{\mathfrak{P}} ;|x|_{\mathfrak{P}} \leq 1\right\} .
$$

L'intersection des éléments de l'ensemble $\mathcal{S}$ avec $\mathcal{O}_{\mathbf{K}}$ est un ensemble qui ne dépend que de $\mathbf{G}$ et du choix du plongement $\Phi$.

Notons $\left(q_{\underline{\lambda}}\right)$ les coefficients du polynôme $Q$. Pour toute place ultramétrique $\mathfrak{P}$ de $\mathbf{L}$, le terme $\left|q_{\underline{\lambda}}\right|_{\mathfrak{P}}$ est majoré (uniformément en $\underline{\lambda}$ ) par

$$
\max _{\underline{\mu}}\left\{\left|p_{\underline{\mu}}\right|_{\mathfrak{P}}\right\} \times \prod_{h=0}^{n} \max \left\{\left|\frac{\varphi_{0}^{(h)}}{\varphi_{\tau_{h}}^{(h)}}\left(m \mathbf{u}_{h}\right)\right|_{\mathfrak{P}}, \ldots,\left|\frac{\varphi_{N_{h}}^{(h)}}{\varphi_{\tau_{h}}^{(h)}}\left(m \mathbf{u}_{h}\right)\right|_{\mathfrak{P}}\right\}^{\mathbf{c}_{h} D_{h}} \times|\mathcal{F}|_{\mathfrak{P}}^{D_{0}+\cdots+D_{n}}
$$

où $|\mathcal{F}|_{\mathfrak{P}}:=\max _{x \in \mathcal{F}}\left\{1,|x|_{\mathfrak{P}}\right\}$ (cette quantité vaut 1 si $\mathfrak{P} \notin \mathcal{S}$ ). Par ailleurs, pour tout $d$-uplet $\underline{h}$ de

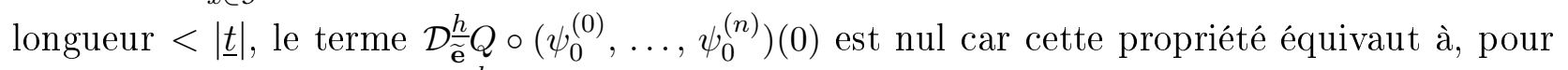
tout $\underline{h}$ de longueur $<|\underline{t}|, \mathcal{D} \underline{\widetilde{\mathbf{e}}} F(m \mathbf{u})=0$, assertion vraie par définition de $\underline{t}$. Par conséquent, le terme

$$
\frac{1}{\underline{t} !} \mathcal{D}_{\widetilde{\mathbf{e}}}^{\underline{\underline{\mathbf{e}}}}\left(Q\left(\psi_{0}^{(0)}, \ldots, \psi_{0}^{(n)}\right)\right)(0)
$$

est le premier* coefficient de Taylor non nul de la fonction holomorphe (au voisinage de 0)

$$
Q\left(\psi_{0}^{(0)}, \ldots, \psi_{0}^{(n)}\right)\left(z_{1} \widetilde{e}_{1}+\cdots+z_{d} \widetilde{e}_{d}\right)
$$

*Pour l'ordre lexicographique sur $\mathbb{N}^{d+1}$. 
L'isomorphisme de schémas formels $\widehat{\mathcal{G}} \longrightarrow$ Specf $A\left[\left[T_{0}, \ldots, T_{d}\right]\right]$ permet d'écrire (formellement) chacune des composantes des fonctions $\psi_{0}^{(h)}$ comme une série formelle $s_{j}^{(h)}$, à coefficients dans $A$, en les variables $T_{0}, \ldots, T_{d}$ (en fait, la structure de groupe produit de $\mathbf{G}$ et le choix de la base $\left(e_{0}, \ldots, e_{d}\right)$ impliquent que seules les variables $T_{\delta_{0}+\cdots+\delta_{h-1}}, \ldots, T_{\delta_{0}+\cdots+\delta_{h}-1}$ entrent en jeu pour $\left.\psi_{0}^{(h)}\right)$. Identifions $\widehat{t}_{\mathcal{G}}\left(=t_{\mathcal{G}} \otimes_{\mathcal{O}_{\text {Spec } A}} \widehat{\mathcal{O}}_{\mathcal{G}}\right)$ à Specf $A\left[\left[z_{0}, \ldots, z_{d}\right]\right]$ grâce au choix de la base e. Les variables $z_{i}$ se relient aux $T_{i}$ via le logarithme formel $: \ell=\left(\ell_{0}, \ldots, \ell_{d}\right)$ (attaché aux choix explicites des isomorphismes $\widehat{\mathcal{G}} \simeq \operatorname{Specf} A\left[\left[T_{0}, \ldots, T_{d}\right]\right]$ et $\left.\widehat{t}_{\mathcal{G}} \simeq \operatorname{Specf} A\left[\left[z_{0}, \ldots, z_{d}\right]\right]\right)$; en effet

$$
z_{i}=\ell_{i}\left(T_{0}, \ldots, T_{d}\right)
$$

avec $\ell_{i}$ indépendant de $T_{0}$ si $i \in\{1, \ldots, d\}$ et $\ell_{0}\left(T_{0}\right)=T_{0}$ (logarithme de $\mathbb{G}_{\mathrm{a}}$ !). De plus, rappelons (voir annexe $\mathrm{C}$ ) que, par construction de ce logarithme, la forme différentielle $\mathrm{d} \ell_{i}$ appartient à

$$
A\left[\left[T_{0}, \ldots, T_{d}\right]\right] . \mathrm{d} T_{0} \oplus \cdots \oplus A\left[\left[T_{0}, \ldots, T_{d}\right]\right] . \mathrm{d} T_{d}
$$

et la matrice jacobienne $\left(\frac{\partial \ell_{i}}{\partial T_{j}}(0)\right)$ est de déterminant égal à 1 . Par conséquent, le premier coefficient de Taylor non nul de

$$
Q\left(\psi_{0}^{(0)}, \ldots, \psi_{0}^{(n)}\right)\left(z_{1} \widetilde{e}_{1}+\cdots+z_{d} \widetilde{e}_{d}\right)
$$

(i.e. (a priori) $\left.\frac{1}{t !} \mathcal{D} \underline{\underline{\mathrm{e}}} Q\left(\psi_{0}^{(0)}, \ldots, \psi_{0}^{(n)}\right)(0)\right)$ est aussi le premier coefficient de Taylor de la série formelle (à coefficients dans $\mathbf{L}_{\mathfrak{P}}$ ) :

$$
Q\left(\ldots,\left(s_{0}^{(h)}, \ldots, s_{N_{h}}^{(h)}\right), \ldots\right)\left(\beta_{1} \ell_{1}+\cdots+\beta_{d} \ell_{d}, T_{1}, \ldots, T_{d}\right)
$$

(si $\widehat{W}$ est le sous-groupe formel de $\widehat{t}_{\mathbf{G}_{\mathbf{L}}}$ d'équation $z_{0}=\beta_{1} z_{1}+\cdots+\beta_{d} z_{d}$, le sous-groupe formel $\ell^{-1}(\widehat{W})$ de $\widehat{\mathbf{G}}_{\mathbf{L}}$ admet pour équation

$$
T_{0}=\beta_{1} \ell_{1}+\cdots+\beta_{d} \ell_{d}
$$

(modulo l'isomorphisme du corollaire I.5.2)). La série

$$
Q\left(\ldots,\left(s_{0}^{(h)}, \ldots, s_{N_{h}}^{(h)}\right), \ldots\right)\left(T_{0}, \ldots, T_{d}\right)
$$

s'écrit

$$
\mathfrak{s}:=\sum_{\underline{\alpha} \in \mathbb{N}^{d+1}} \theta_{\underline{\alpha}} \cdot T_{0}^{\alpha_{0}} \cdots T_{d}^{\alpha_{d}}
$$

avec $\theta_{\underline{\alpha}} \in \sum_{\underline{\lambda}} A$. $q_{\underline{\lambda}}$ et $\theta_{\underline{\alpha}}=0$ si $\alpha_{0}>D_{0}$. Écrivons également

$$
\ell_{i}=\sum_{\underline{n} \in \mathbb{N}^{d+1}} a_{\underline{n}}^{(i)} T_{0}^{n_{0}} \cdots T_{d}^{n_{d}}
$$

pour $i \in\{1, \ldots, d\}\left(a_{\underline{n}}^{(i)} \in \mathbf{K}\right)$. Posons $\gamma_{\underline{n}}:=\sum_{i=1}^{d} \beta_{i} a_{\underline{n}}^{(i)}$. Alors

$$
\mathfrak{s}\left(\beta_{1} \ell_{1}+\cdots+\beta_{d} \ell_{d}, T_{1}, \ldots, T_{d}\right)
$$

est égal à

$$
\sum_{\alpha_{0} \leq D_{0}} \theta_{\underline{\alpha}}\left(\sum_{\underline{n} \in \mathbb{N}^{d}} \gamma_{\underline{n}} \mathbf{T}^{\underline{n}}\right)^{\alpha_{0}} T_{1}^{\alpha_{1}} \cdots T_{d}^{\alpha_{d}}
$$


et le coefficient devant $T_{1}^{t_{1}} \cdots T_{d}^{t_{d}}$ est une somme de termes de la forme :

$$
\theta_{\underline{\alpha}} \gamma_{\underline{n}_{1}} \cdots \gamma_{\underline{n}_{0}} .
$$

Comme, pour tout $j, \mathrm{~d} \ell_{j}$ est une forme différentielle formelle à coefficients dans $\mathcal{O}_{K_{\mathrm{p}}}$, le coefficient $a_{\underline{n}}^{(j)}$ est une somme de termes de la forme $a / m$, où $a \in \mathcal{O}_{K_{\mathbf{p}}}$ et $m$ est un entier égal à une des composantes de $\underline{n}$. Par conséquent, le terme $\theta_{\underline{\alpha}} \gamma_{\underline{n}_{1}} \cdots \gamma_{\underline{n}_{\alpha_{0}}}$ est une somme de termes de la forme $a^{\prime} /\left(m_{1} \ldots m_{\alpha_{0}}\right)$, où $a^{\prime} \in \mathcal{O}_{K_{\mathbf{p}}}$ et $m_{1}, \ldots, m_{\alpha_{0}}$ sont des entiers non nuls, dont la somme est $\leq|\underline{n}|$. Par conséquent, la quantité

$$
\delta_{|\underline{\mid t}|}\left(D_{0}\right) \cdot \theta_{\underline{\alpha}} \gamma_{\underline{n}_{1}} \ldots \gamma_{\underline{n}_{\alpha_{0}}}
$$

(pour $\underline{n}_{1}+\cdots+\underline{n}_{\alpha_{0}} \leq \underline{t}$, au sens lexicographique) est un polynôme en $\beta_{1}, \ldots, \beta_{d}$, à coefficients dans $A$, de degré $\leq D_{0}$ (car $\left.\alpha_{0} \leq D_{0}\right)$. L'estimation $\mathfrak{P}$-adique $\left(\mathfrak{P} \in M_{\mathbf{L}, f} \backslash \mathcal{S}\right)$ du coefficient de Taylor

$$
\frac{1}{\underline{t} !} \mathcal{D} \underset{\widetilde{\mathbf{e}}}{\underline{\mathrm{e}}} Q\left(\psi_{0}^{(0)}, \ldots, \psi_{0}^{(n)}\right)(0)
$$

est alors immédiate :

$$
\left|\frac{1}{\underline{t} !} \mathcal{D}_{\underline{\tilde{\mathbf{e}}}}^{\underline{t}} Q\left(\psi_{0}^{(0)}, \ldots, \psi_{0}^{(n)}\right)(0)\right|_{\mathfrak{P}} \leq\left|\frac{1}{\delta_{|\underline{t \mid}|}\left(D_{0}\right)}\right|_{\mathfrak{P}} \max \left\{1,\left|\beta_{1}\right|_{\mathfrak{P}}, \ldots,\left|\beta_{d}\right|_{\mathfrak{P}}\right\}^{D_{0}} \max _{\underline{\underline{\lambda}}}\left\{\left|q_{\underline{\lambda}}\right|_{\mathfrak{P}}\right\}
$$

majoration de laquelle se déduit (40) via l'égalité (41) et la majoration (42) des coefficients $q_{\underline{\lambda}}$.

Si $\mathfrak{P} \in \mathcal{S}$, le procédé ci-dessus ne permet pas d'obtenir directement une majoration (même plus faible) de

$$
\left|\frac{1}{\underline{t} !} \mathcal{D}_{\widetilde{\mathbf{e}}}^{\underline{t}} Q\left(\psi_{0}^{(0)} \cdots \psi_{0}^{(n)}\right)(0)\right|_{\mathfrak{P}},
$$

car on ne connaît pas précisément la dépendance en $r$ de ce terme. Dans ce cas, nous disposons cependant d'une majoration similaire plus faible :

Lemme I.14.6. Avec les notations du lemme précédent, il existe une constante $c_{38}$ ne dépendant que de $(\mathbf{G}, \Phi)$, telle que, pour toute place $\mathfrak{P} \in \mathcal{S}$, on ait :

$$
\begin{aligned}
& \left|\frac{1}{\prod_{j=0}^{n}\left(\varphi_{\tau_{j}}^{(j)}\left(m \mathbf{u}_{j}\right)\right)^{D_{j}}} \frac{1}{\underline{t} !} \mathcal{D} \underline{\underline{\underline{\mathbf{e}}}} F(m \mathbf{u})\right|_{\mathfrak{P}} \leq c_{38}^{T} \times \max \left\{1,\left|\beta_{1}\right|_{\mathfrak{P}}, \ldots,\left|\beta_{d}\right|_{\mathfrak{P}}\right\}^{D_{0}} \\
& \times \max _{\underline{\lambda}}\left\{\left.\left|p_{\underline{\lambda}}\right|\right|_{\mathfrak{P}}\right\} \times \prod_{h=0}^{n} \frac{1}{\left|A_{\tau_{h}}^{(h)}\left(\psi_{0}^{(h)}(0), \psi_{\tau_{h}}^{(h)}\left(m \mathbf{u}_{h}\right)\right)\right|_{\mathfrak{P}}^{D_{h}}} \\
& \times \prod_{j=0}^{n} \max \left\{\left|\frac{\varphi_{0}^{(j)}\left(m \mathbf{u}_{j}\right)}{\varphi_{\tau_{j}}^{(j)}\left(m \mathbf{u}_{j}\right)}\right|_{\mathfrak{P}}, \cdots,\left|\frac{\varphi_{N_{j}}^{(j)}\left(m \mathbf{u}_{j}\right)}{\varphi_{\tau_{j}}^{(j)}\left(m \mathbf{u}_{j}\right)}\right|_{\mathfrak{P}}\right\}^{\mathbf{c}_{j} D_{j}} .
\end{aligned}
$$


Démonstration. Pour contourner le problème évoqué en préambule du lemme, nous allons majorer 《trivialement »ce coefficient grâce à la propriété de différentiabilité des fonctions $\varphi_{j}^{(h)} / \varphi_{0}^{(h)}(c f . \S$ I.5). Reprenons les notations introduites dans la preuve précédente. Il existe un polynôme $C_{k j}$ ne dépendant que de $\mathbf{G}$ tel que

$$
\frac{\partial}{\partial z_{k}}\left(\frac{\varphi_{j}^{(h)}}{\varphi_{0}^{(h)}}\right)=C_{k j}\left(\psi_{0}^{(0)}, \ldots, \psi_{0}^{(n)}\right) .
$$

Nous développons

$$
\mathcal{D}_{\widetilde{\mathbf{e}}}^{t}=\sum_{0 \leq m_{i} \leq t_{i}}\left(\prod_{i=1}^{d}\left(\begin{array}{c}
t_{i} \\
m_{i}
\end{array}\right) \cdot \beta_{i}^{m_{i}} \cdot\left(\frac{\partial}{\partial z_{i}}\right)^{t_{i}-m_{i}}\right)\left(\frac{\partial}{\partial z_{0}}\right)^{m_{1}+\cdots+m_{d}}
$$

Appliquée à $Q\left(\psi_{0}^{(0)}, \ldots, \psi_{0}^{(n)}\right)$, la somme se restreint à $m_{1}+\cdots+m_{d} \leq D_{0}$ car la fonction $Q\left(\psi_{0}^{(0)}, \ldots, \psi_{0}^{(n)}\right)$ est polynomiale en la variable $z_{0}$ de degré $\leq D_{0}$. Utilisons récursivement les égalités du type (44); il vient :

$$
\left|\mathcal{D} \frac{\underline{\underline{\mathbf{e}}}}{\left(\psi_{0}\right.}\left(\psi_{0}^{(0)}, \ldots, \psi_{0}^{(n)}\right)(0)\right|_{\mathfrak{P}} \leq|\mathcal{F}|_{\mathfrak{P}}^{|\underline{t}|+D_{0}+\cdots+D_{n}} \times \max _{\underline{\underline{\lambda}}}\left\{\left|q_{\underline{\lambda}}\right|_{\mathfrak{P}}\right\} \times \max \left\{1,\left|\beta_{1}\right|_{\mathfrak{P}}, \ldots,\left|\beta_{d}\right|_{\mathfrak{P}}\right\}^{D_{0}} .
$$

Compte tenu de l'évaluation $\mathfrak{P}$-adique de $\frac{1}{\underline{t} !}$ (une exponentielle de $|\underline{t}|$ ), on obtient le résultat annoncé, grâce au majorant (42).

\section{$\S 15$. Estimations archimédiennes}

Lemme I.15.1. Avec les notations du lemme I.14.5, il existe une constante $c_{39}$ ne dépendant que de $(\mathbf{G}, \Phi)$ telle que, pour toute place archimédienne $\sigma: \mathbf{L} \rightarrow \mathbb{C}$, on ait

$$
\begin{aligned}
\left|\frac{1}{\prod_{j=0}^{n}\left(\varphi_{\tau_{j}}^{(j)}\left(m \mathbf{u}_{j}\right)\right)^{D_{j}} \frac{1}{\underline{t}} \mathcal{D}} \mathcal{D}_{\underline{\underline{\mathbf{e}}}}^{\underline{t}} F(m \mathbf{u})\right|_{\sigma} \leq & c_{39}^{T} \times \max \left\{1,\left|\beta_{1}\right|_{\sigma}, \ldots,\left|\beta_{d}\right|_{\sigma}\right\}^{D_{0}} \times \max _{\underline{\lambda}}\left\{\left|p_{\underline{\lambda}}\right|_{\sigma}\right\} \\
& \times \prod_{h=0}^{n} \frac{1}{\left|A_{\tau_{h}}^{(h)}\left(\psi_{0}^{(h)}(0), \psi_{\tau_{h}}^{(h)}\left(m \mathbf{u}_{h}\right)\right)\right|_{\sigma}^{D_{h}}} \\
& \times \prod_{j=0}^{n} \max \left\{\left|\frac{\varphi_{0}^{(j)}\left(m \mathbf{u}_{j}\right)}{\varphi_{\tau_{j}}^{(j)}\left(m \mathbf{u}_{j}\right)}\right|_{\sigma}, \cdots,\left|\frac{\varphi_{N_{j}}^{(j)}\left(m \mathbf{u}_{j}\right)}{\varphi_{\tau_{j}}^{(j)}\left(m \mathbf{u}_{j}\right)}\right|_{\sigma}\right\}^{\mathbf{c}_{j} D_{j}}
\end{aligned}
$$

Démonstration. C'est exactement la même démonstration que celle du paragraphe précédent pour les places $\mathfrak{P} \in \mathcal{S}$. La constante $c_{39}$ n'est pas tout à fait $\max _{\sigma}\left\{|\mathcal{F}|_{\sigma}\right\}^{n+2}$ car, contrairement au cas ultramétrique où la valeur absolue d'une somme est majorée par le maximum des termes de la somme, nous devons tenir compte de la longueur des polynômes $A_{\tau_{h}}^{(h)}$ et $C_{k j}$ (notamment dans la majoration de $\left.\left|q_{\underline{\lambda}}\right|_{\sigma}\right)$. 


\section{$\S$ 16. Conclusion}

Des estimations $\mathfrak{P}$-adiques et archimédiennes du nombre algébrique

$$
\frac{1}{\prod_{j=0}^{n}\left(\varphi_{\tau_{j}}^{(j)}\left(m \mathbf{u}_{j}\right)\right)^{D_{j}}} \frac{1}{\underline{t} !} \mathcal{D} \frac{\underline{\underline{\mathbf{e}}}}{\mathrm{e}} F(m \mathbf{u}),
$$

nous allons en déduire une minoration de $\left|\frac{1}{t \underline{t} !} \mathcal{D} \underline{\underline{\mathbf{e}}} F(m \mathbf{u})\right|$. La comparaison avec la majoration de ce complexe obtenue à la suite de l'extrapolation (corollaire I.13.4) donnera une contradiction. Nous en déduirons alors les théorèmes I.3.1 et I.3.2.

Appliquons la formule du produit au nombre (46). Nous obtenons l'inégalité suivante :

$$
\begin{aligned}
& \frac{1}{D} \log \left|\frac{1}{\underline{t} !} \mathcal{D}_{\widetilde{\mathbf{e}}}^{\underline{t}} F(m \mathbf{u})\right| \geq \\
& -\left(c_{40} T+D_{0} h(W)+h(P)+\log \delta_{|\underline{t}|}\left(D_{0}\right)+\frac{1}{D} \log \prod_{h=0}^{n}\left|A_{\tau_{h}}^{(h)}\left(\psi_{0}^{(h)}(0), \psi_{\tau_{h}}^{(h)}\left(m \mathbf{u}_{h}\right)\right)\right|^{D_{h}}\right. \\
& \left.\quad+\sum_{j=0}^{n} \mathbf{c}_{j} D_{j}\left\{\frac{1}{D} \sum_{v \neq \sigma_{0}} \log \max \left\{\left|\frac{\varphi_{0}^{(j)}(m \mathbf{u})}{\varphi_{\tau_{j}}^{(j)}(m \mathbf{u})}\right|_{v}, \cdots,\left|\frac{\varphi_{N_{j}}^{(j)}(m \mathbf{u})}{\varphi_{\tau_{j}}^{(j)}(m \mathbf{u})}\right|_{v}\right\}\right\}\right)
\end{aligned}
$$

(la dernière somme porte sur toutes les places (finies et infinies) de $\mathbf{L}$, différentes de $\sigma_{0}$ ). La définition des polynômes $A_{\tau_{h}}^{(h)}$ implique l'existence d'une constante $c_{41}$, ne dépendant que de $(\mathbf{G}, \Phi)$, telle que, pour tout $h \in\{0, \ldots, n\}$,

$$
\left|A_{\tau_{h}}^{(h)}\left(\psi_{0}^{(h)}(0), \psi_{\tau_{h}}^{(h)}\left(m \mathbf{u}_{h}\right)\right)\right| \leq c_{41} \max \left\{\left|\frac{\varphi_{0}^{(h)}(m \mathbf{u})}{\varphi_{\tau_{h}}^{(h)}(m \mathbf{u})}\right|, \cdots,\left|\frac{\varphi_{N_{h}}^{(h)}(m \mathbf{u})}{\varphi_{\tau_{h}}^{(h)}(m \mathbf{u})}\right|\right\}^{\mathbf{c}_{h}} .
$$

Le minorant de l'inégalité (47) se simplifie alors et peut être remplacé par

$$
-c_{42}\left\{T \log D_{0}+D_{0} h(W)+h(P)+\sum_{j=0}^{n} D_{j} h\left(m \mathbf{p}_{j}\right)\right\} .
$$

Choisissons un $n$-uplet $\left(\tau_{1}, \ldots, \tau_{n}\right)$ tel que

$$
\begin{aligned}
\left|\varphi_{\tau_{j}}^{(j)}(m \mathbf{u})\right| & =\max \left\{\left|\varphi_{0}^{(j)}(m \mathbf{u})\right|, \ldots,\left|\varphi_{N_{j}}^{(j)}(m \mathbf{u})\right|\right\} \\
& \geq \exp \left\{-c_{j}^{-}\left(1+m\left\|\mathbf{u}_{j}\right\|\right)^{\rho_{j}}\right\} \text { lorsque } j \neq 0
\end{aligned}
$$

et

$$
\left|\varphi_{\tau_{0}}^{(0)}(m \mathbf{u})\right| \geq 1
$$

La quantité $\log \left|\frac{1}{\underline{t} !} \mathcal{D} \underset{\widetilde{\mathbf{e}}}{t} F(m \mathbf{u})\right|$ est alors minorée par

$$
\begin{aligned}
& -c_{43}\left\{D T \log D_{0}+D_{0}\left(D h(W)+\max _{0 \leq s \leq(d+1) S}\left\{D h\left(s u_{0}\right)\right\}\right)\right. \\
& \left.+D h(P)+\sum_{j=1}^{n} D_{j}\left(\max _{0 \leq s \leq(d+1) S}\left\{D h\left(s \mathbf{p}_{j}\right)\right\}+\left(S\left\|\mathbf{u}_{j}\right\|\right)^{\rho_{j}}\right)\right\} .
\end{aligned}
$$


En remplaçant les paramètres par leur valeur, nous obtenons

$$
\log \left|\frac{1}{\underline{t} !} \mathcal{D} \underline{\underline{\mathbf{e}}} F(m \mathbf{u})\right| \geq-c_{44} C_{0}^{1 / 2} U_{0} .
$$

Ce qui contredit, pour $C_{0}$ assez grand, la majoration du corollaire I.13.4 (page 36). Ainsi, une des trois hypothèses* émises lors de la démonstration est fausse. Donc

1. Ou bien l'inégalité (33) est fausse et alors

$$
\log |\Lambda| \geq-C_{0} U_{0}
$$

2. Ou bien $\mathbf{u} \in t_{\widetilde{\mathbf{G}}}(\mathbb{C})$ (et nécessairement nous sommes dans le cas périodique). Le théorème se déduit alors du lemme I.9.4.

3. Ou bien $\mathbf{u} \notin t_{\widetilde{\mathbf{G}}}(\mathbb{C})$ et nous sommes dans le cas périodique. Alors

$$
|\Lambda| \geq \frac{1}{2} \mathrm{~d}\left(\mathbf{u}, t_{\widetilde{\mathbf{G}}}(\mathbb{C})\right)
$$

ce qui donne, d'après la première partie du corollaire I.9.5, l'inégalité

$$
\begin{aligned}
\log |\Lambda| & \geq-\log 2+\log \left[C_{0}^{-23 d}\left(D+D h\left(\mathbf{p}_{1}\right)+\cdots+D h\left(\mathbf{p}_{n}\right)+E\left\|\mathbf{u}_{1}\right\|+\cdots+E\left\|\mathbf{u}_{n}\right\|\right)^{-20 d}\right] \\
& \geq-U_{0}
\end{aligned}
$$

Pour obtenir le théorème I.3.1, il ne reste plus qu'à remplacer dans $U_{0}$, l'entier $S$ par a, la comparaison entre $|\Lambda|$ et $\mathrm{d}(\mathbf{u}, W)$ s'effectuant via l'égalité (1). Quant au théorème I.3.2, les hypothèses de ce théorème impliquent que nous sommes nécessairement dans le cas nonpériodique. Tous les calculs effectués dans ce cas restent vrais lorsque $U_{0}$ est remplacé par $U_{0}^{\prime}$. La seule modification notable se situe au niveau du lemme de zéros, en particulier lors du corollaire I.11.2, où nous invitons le lecteur à se reporter pour de plus amples détails.

*Deux de ces hypothèses sont dans le corollaire I.9.5 (page 25), la troisième est au début du § I.13 (page 34 ). 


\section{Annexe A}

\section{Paramètres libres}

Dans cette partie, nous reprenons les énoncés des lemmes et propositions qui conduisent aux théorèmes I.3.1 et I.3.2 mais sans choisir les paramètres $\widetilde{D}_{0}, \ldots, \widetilde{D}_{n}, T, S, x_{0}$ comme au début du paragraphe I.7. Le but est de laisser au lecteur les clefs de la démonstration des théorèmes et la possibilité de changer les paramètres sans avoir à refaire tous les calculs. Nous obtenons alors un théorème qui est, en quelque sorte, une « boîte noire » dans laquelle, en entrée, on choisit des paramètres, et qui donne, en sortie, une minoration de la distance $\mathrm{d}(\mathbf{u}, W)$ de $\mathbf{u}$ à l'hyperplan $W$. En déconstruisant les inégalités données par ce théorème, nous énonçons les contraintes sur les paramètres puis nous expliquons pourquoi notre choix de paramètres est «le bon ».

\section{$\S$ A.1. Les paramètres}

Considérons $x, x_{0}, \widetilde{D}_{0}, \ldots, \widetilde{D}_{n}, \widetilde{T}_{0}<\widetilde{T}$ des réels strictement positifs, $S_{0}<S$ des entiers naturels non nuls. Posons $D_{i}^{\#}=x \widetilde{D}_{i}, D_{i}=\left[D_{i}^{\#}\right], T^{\#}=x_{0} \widetilde{T}, T=\left[T^{\#}\right], T_{0}^{\#}=x_{0} \widetilde{T}_{0}, T_{0}=\left[T_{0}^{\#}\right]$.

Nous allons donner des conditions (hypothèses A.1.1 et A.1.3 ci-dessous) que doivent vérifier ces paramètres pour que n'apparaisse pas de « sous-groupe obstructeur ${ }^{*}$.

Tout d'abord, supposons

Hypothèse A.1.1.

$$
x_{0} \geq \frac{(\widetilde{T})^{d} \operatorname{card}\left(\Gamma_{\mathbf{p}}(S)\right)}{C_{0}\left(\operatorname{deg}_{\Phi} \mathbf{G}\right) \widetilde{D}_{0}^{\delta_{0}} \cdots \widetilde{D}_{n}^{\delta_{n}}} .
$$

Remarque : c'est cette condition qui justifie le choix de $U_{0}$ sous forme de produit (p. 18). Cette hypothèse permet d'obtenir le pendant de la proposition I.7.1 :

Proposition A.1.2 (Proposition I.7.1 bis). Il existe un réel $\left.x \in] 0, x_{0}\right]$ tel que les conditions suivantes soient vérifiées.

(i) Pour tout sous-groupe connexe $\mathbf{G}^{\prime}$ de $\mathbf{G}$ de codimension $r^{\prime}$, dont l'espace tangent à l'origine est inclus dans l'hyperplan $W$, on $a$ :

$$
\left(T^{\#}\right)^{r^{\prime}-1} \times \operatorname{card}\left(\frac{\Gamma_{\mathbf{p}}(S)+\mathbf{G}^{\prime}(\mathbf{L})}{\mathbf{G}^{\prime}(\mathbf{L})}\right) \times \mathscr{H}\left(\mathbf{G}^{\prime} ; D_{0}^{\#}, \ldots, D_{n}^{\#}\right) \geq C_{0} \mathscr{H}\left(\mathbf{G} ; D_{0}^{\#}, \ldots, D_{n}^{\#}\right) .
$$

*Rappelons que, dans ce texte, la terminologie « sous-groupe obstructeur » désigne simplement un sousgroupe de $\mathbf{G}$ donné par le lemme de zéros de Philippon [63]. 
(ii) Il y a égalité pour un certain groupe que l'on notera $\widetilde{\mathbf{G}}$.

On notera que $T^{\#}$ a remplacé $\widetilde{T}$. Comme dans la proposition I.7.1, le point essentiel de la démonstration est que, si on note

$$
B\left(\mathbf{G}^{\prime}\right)=A\left(\mathbf{G}^{\prime}\right)^{1 / r^{\prime}} \max \left\{A\left(\mathbf{G}^{\prime}\right), x_{0}\right\}^{\frac{r^{\prime}-1}{r^{\prime}}},
$$

alors, par construction, $x \leq B(\{0\})$ et le calcul de $B(\{0\})$ repose sur l'inégalité $x_{0} \geq A(\{0\})$, qui est l'hypothèse A.1.1 (pour plus de détails, cf. page 20). L'hypothèse A.1.1 valide donc la proposition A.1.2 dont le rôle est d'éliminer les « sous-groupes obstructeurs » d'espace tangent inclus dans $W$.

Afin de faire disparaître, parmi ces sous-groupes, ceux dont l'espace tangent à l'origine n'est pas inclus dans $W$, on suppose*

\section{Hypothèse A.1.3.}

$$
\widetilde{T}_{0}>c_{45} \times\left(\max \left\{\widetilde{D}_{0}, \ldots, \widetilde{D}_{n}\right\}+\frac{2}{x_{0}}\right) .
$$

En effet, si un polynôme multihomogène de multidegré $\left(D_{0}, \ldots, D_{n}\right)$, dont la restriction à $\mathbf{G}$ n'est pas identiquement nulle, s'annule à l'ordre $T$ le long de $W$ en les points $\left\{0_{\mathbf{G}}, \mathbf{p}, \ldots,(d+1) S \mathbf{p}\right\}$ alors il existe un sous-groupe (《obstructeur $\left.»\right) \mathbf{G}^{\prime} \subsetneq \mathbf{G}$ vérifiant l'inégalité :

$$
T^{\lambda} \times \operatorname{card}\left(\frac{\Gamma_{\mathbf{p}}(S)+\mathbf{G}^{\prime}(\mathbf{L})}{\mathbf{G}^{\prime}(\mathbf{L})}\right) \times \mathscr{H}\left(\mathbf{G}^{\prime} ; D_{0}^{\prime}, \ldots, D_{n}^{\prime}\right) \leq c_{46} \mathscr{H}\left(\mathbf{G} ; D_{0}^{\prime}, \ldots, D_{n}^{\prime}\right)
$$

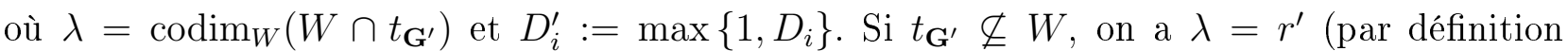
$r^{\prime}:=\operatorname{codim}_{\mathbf{G}} \mathrm{G}^{\prime}$ ) et de l'inégalité (A.2) nous déduisons

$$
T^{r^{\prime}} \leq c_{46} \frac{\mathscr{H}\left(\mathbf{G} ; D_{0}^{\prime}, \ldots, D_{n}^{\prime}\right)}{\mathscr{H}\left(\mathbf{G}^{\prime} ; D_{0}^{\prime}, \ldots, D_{n}^{\prime}\right)} \leq c_{46} \times \operatorname{deg}_{\Phi} \mathbf{G} \times \max \left\{D_{0}^{\prime}, \ldots, D_{n}^{\prime}\right\}^{r^{\prime}}
$$

i.e.

$$
\left(\frac{T}{\max \left\{D_{0}^{\prime}, \ldots, D_{n}^{\prime}\right\}}\right)^{r^{\prime}} \leq c_{46} \operatorname{deg}_{\Phi} \mathbf{G} .
$$

Comme $r^{\prime}>0\left(\right.$ car $\left.\mathbf{G}^{\prime} \subsetneq \mathbf{G}\right)$, cela implique

$$
\frac{T}{\max \left\{D_{0}^{\prime}, \ldots, D_{n}^{\prime}\right\}} \leq c_{46} \operatorname{deg}_{\Phi} \mathbf{G}
$$

puis

$$
x_{0} \widetilde{T}-1<\left[x_{0} \widetilde{T}\right]=T \leq c_{46} \operatorname{deg}_{\Phi} \mathbf{G} \times\left(1+x \cdot \max \left\{\widetilde{D}_{0}, \ldots, \widetilde{D}_{n}\right\}\right) .
$$

Comme $x \leq x_{0}$, l'hypothèse A.1.3 entre en contradiction avec l'inégalité ci-dessus, ce qui impose à $\mathbf{G}^{\prime}$ d'avoir un espace tangent inclus dans $W$.

Remarque : si $u_{0} \neq 0$ et si $\prod_{j=1}^{n} \mathbf{G}_{j}$ est une variété semi-abélienne, nous pouvons remplacer l'hypothèse A.1.3 par l'hypothèse

$$
\widetilde{T}_{0} \geq c_{47} \times\left(\max \left\{\frac{\widetilde{D}_{0}}{S_{0}}, \widetilde{D}_{1}, \ldots, \widetilde{D}_{n}\right\}+\frac{2}{x_{0}}\right) .
$$

*Dans les cas particuliers où nous disposons d'une description assez fine des sous-groupes obtructeurs, cette hypothèse n'est pas indispensable ( $c f$. par exemple [1] et la remarque de la fin de ce paragraphe). 


\section{$\S$ A.2. Le cas périodique : compléments}

Nous écrivons ici l'énoncé général du lemme I.9.4 :

\section{Lemme A.2.1.}

$$
\operatorname{deg}_{\Phi} \pi(\widetilde{\mathbf{G}}) \leq\left(\operatorname{deg}_{\Phi} \mathbf{G}\right) \times C_{0} \times \frac{D_{0}^{\#}}{\operatorname{card}\left(\frac{\Gamma_{\mathbf{p}}(S)+\widetilde{\mathbf{G}}(\mathbf{L})}{\widetilde{\mathbf{G}}(\mathbf{L})}\right)} \times \max \left\{\left(\frac{\widetilde{D}_{j_{1}}}{\widetilde{T}}\right) \times \cdots \times\left(\frac{\widetilde{D}_{\tilde{r}_{\tilde{r}-1}}}{\widetilde{T}}\right)\right\}
$$

où le maximum porte sur les $(\widetilde{r}-1)$-uplets $\left(j_{1}, \ldots, j_{\widetilde{r}-1}\right)$ vérifiant $1 \leq j_{1} \leq \cdots \leq j_{\widetilde{r}-1} \leq n$ et tels que chaque $j_{m}$ ne soit répété qu'au plus $\delta_{j_{m}}$ fois*.

La démonstration découle instantanément de celle du lemme I.9.4, en remplaçant $\widetilde{T}$ par $T^{\#}$. Pour obtenir l'équivalent du corollaire I.9.5, nous supposerons

Hypothèse A.2.2. $\mathbf{u} \notin t_{\widetilde{\mathbf{G}}}(\mathbb{C})$ et $|\Lambda|<\frac{1}{2} \mathrm{~d}\left(\mathbf{u}, t_{\widetilde{\mathbf{G}}}(\mathbb{C})\right)$ dans le cas périodique (où $\mathrm{d}$ est la distance associée à la norme $\|$.$\| , définie au \S$ I.5).

Corollaire A.2.3. Dans le cas périodique, pour $C_{0} \geq c_{48}$, on a

$$
\mathrm{d}\left(\mathbf{u}, t_{\widetilde{\mathbf{G}}}(\mathbb{C})\right) \geq \frac{1}{C_{0}^{2}} \times \frac{1}{D_{0}^{\#}} \times\left(\frac{\widetilde{T}}{\max _{1 \leq i \leq n} \widetilde{D}_{i}}\right)^{\widetilde{r}-1}
$$

De plus $\mathbf{w} \notin t_{\widetilde{\mathbf{G}}}(\mathbb{C})$ et $t_{\widetilde{\mathbf{G}}}(\mathbb{C}) \subsetneq W \otimes \mathbb{C}$. En particulier $w_{d} \neq 0$.

Démonstration. Voir la démonstration du corollaire I.9.5, page 25.

Le corollaire I.9.8 devient :

Corollaire A.2.4. Dans le cas périodique, on a

$$
\left|w_{d}\right| \geq \frac{1}{C_{0}^{3}} \times \frac{1}{D_{0}^{\#}} \times\left(\frac{\widetilde{T}}{\max _{1 \leq i \leq n} \widetilde{D}_{i}}\right)^{\widetilde{r}-1}
$$

La remarque suivant la proposition I.10.2 devient : Remarque :

$$
\frac{1+\left|w_{1}\right|+\cdots+\left|w_{d-1}\right|}{\left|w_{d}\right|} \leq 2(d+1)\left(\frac{\sqrt{d}}{2}+C_{0}^{2}(1+\|\mathbf{u}\|) D_{0}^{\#}\left(\frac{\max _{1 \leq i \leq n} \widetilde{D}_{i}}{\widetilde{T}}\right)^{\widetilde{r}-1}\right)
$$

${ }^{*}$ Nous pourrions préciser un peu plus ce point en considérant, au lieu de $\delta_{j_{m}}$, le degré en la variable $X_{j_{m}}$ du polynôme $\mathscr{H}\left(\widetilde{\mathbf{G}} ; X_{0}, \ldots, X_{n}\right)$. On note traditionnellement $e_{j_{m}}(\mathcal{I}(\widetilde{\mathbf{G}}))$ ce degré $(c f .[69])$. 


\section{$\S$ A.3. Rang du système linéaire}

Lemme A.3.1. Soit $\rho$ le rang du système (27). Il existe une constante $c_{49}$, ne dépendant que de $(\mathbf{G}, \Phi)$, telle que

$$
\rho \leq c_{49} C_{0} \cdot \max \left\{\frac{1+S_{0}}{1+S}, \frac{T_{0}}{T}\right\} \cdot \mathscr{H}\left(\mathbf{G} ; D_{0}^{\prime}, \ldots, D_{n}^{\prime}\right) \text { et } \nu \geq c_{49} D \mathscr{H}\left(\mathbf{G} ; D_{0}^{\prime}, \ldots, D_{n}^{\prime}\right)
$$

où $D_{i}^{\prime}:=\max \left\{1, D_{i}\right\}$.

Nous supposerons

Hypothèse A.3.2.

$$
\max \left\{\frac{1+S_{0}}{1+S}, \frac{T_{0}}{T}\right\} \leq \frac{1}{C_{0}^{2}}
$$

La démonstration de la non-nullité des entiers $D_{i}$ (corollaire I.11.2) repose encore sur l'argument donné page 28 et l'hypothèse A.1.3.

\section{$\S$ A.4. Construction de la fonction auxiliaire}

Notons $\xi_{1}, \ldots, \xi_{D}$ une base quelconque du $\mathbb{Q}$-espace vectoriel L. La version brute de la proposition I.12.3 est :

Proposition A.4.1. Soit $p$ un réel strictement positif. Il existe une constante $c_{50}$ ne dépendant que du couple $(\mathbf{G}, \Phi)$ et il existe un polynôme multihomogène $P$ de $\mathbf{L}[\mathbb{P}]$, de multidegré $\left(D_{0}, \ldots, D_{n}\right)$, ne s'annulant pas identiquement sur $\mathbf{G}$, tel que :

- Les coefficients de $P$ appartiennent à $\mathbb{Z} . \xi_{1} \oplus \cdots \oplus \mathbb{Z} . \xi_{D}$

- La hauteur $h(P)$ est majorée par :

$$
\frac{c_{50}}{C_{0} D}\left\{T+p+\digamma+D_{0} \log \left(e+S\left\|\mathbf{u}_{0}\right\|\right)+\sum_{j=1}^{n} D_{j}\left(1+S\left\|\mathbf{u}_{j}\right\|\right)^{\rho_{j}}\right\}+c_{50} h\left(\xi_{1}: \cdots: \xi_{D}\right)+\log D
$$

où

$$
\digamma= \begin{cases}0 & \text { dans le cas non-périodique, } \\ T \log (1+\|\mathbf{u}\|) & \text { dans le cas périodique. }\end{cases}
$$

- Pour chaque $(\underline{t}, s) \in \Upsilon$, la valeur absolue du coefficient de Taylor $\frac{1}{t !} \mathcal{D}_{\mathbf{f}}^{\underline{t}} F($ su) (où, nous le rappelons, $F=P \circ \Phi$ ) est majorée par $e^{-p}$.

Remarque : la conclusion nous donnera la valeur optimale de $p$ (à une constante près), fonction du cas - périodique, non-périodique - dans lequel nous sommes.

Démonstration. Comme dans la preuve de la proposition I.12.3, on écrit

$$
P=\sum_{\underline{\lambda}, i} a_{\underline{\lambda}, i} \cdot \xi_{i} \cdot \mathbf{X}^{\underline{\lambda}}
$$


et on pose

$$
u_{(\underline{\lambda}, i)(\underline{t}, s)}=\xi_{i} \frac{1}{\underline{t} !} \mathcal{D}_{\overline{\mathbf{f}}}^{\underline{t}}\left(\Phi^{\underline{\lambda}}\right)(s \mathbf{u}) .
$$

Le lemme I.12.2 implique l'existence d'une constante $c_{51}$ ne dépendant que de $(\mathbf{G}$, $\Phi)$, telle que

$$
\begin{aligned}
\max _{(\underline{t}, s) \in \Upsilon}\left\{\sum_{(\underline{\lambda}, i)}\left|u_{(\underline{\lambda}, i),(\underline{t}, s)}\right|\right\} \leq & c_{51}^{T+D_{0}} \times e^{D h\left(\xi_{1} \cdots \cdots: \xi_{D}\right)+\log D} \\
& \times\left(1+S\left\|\mathbf{u}_{0}\right\|\right)^{D_{0}} \times \exp \left\{c \sum_{j=1}^{n} D_{j}\left(1+S\left\|\mathbf{u}_{j}\right\|_{j}\right)^{\rho_{j}}\right\} \\
& \times \begin{cases}1 & \text { cas non-périodique } \\
(1+\|\mathbf{u}\|)^{2(d+1) T} & \text { cas périodique }\end{cases}
\end{aligned}
$$

Puis, pour $C_{0}$ assez grand, la majoration (A.5) et le lemme de Thue-Siegel (lemme I.12.1, page 29) induisent l'existence des entiers $a_{\underline{\lambda}, i}$, de module inférieur à $e^{\delta}$ où

$$
\begin{aligned}
\delta:=\frac{c_{52}}{C_{0} D}\{T & +\log S+p+\digamma+D+D_{0} \log \left(1+S\left\|\mathbf{u}_{0}\right\|\right) \\
& \left.+\sum_{j=1}^{n} D_{j}\left(1+S\left\|\mathbf{u}_{j}\right\|\right)^{\rho_{j}}+D h\left(\xi_{1}: \cdots: \xi_{D}\right)+\log D\right\}
\end{aligned}
$$

L'inégalité $h(P) \leq \log \left(D e^{\delta}\right)+h\left(\xi_{1}: \cdots: \xi_{D}\right)$ permet de conclure la démonstration.

\section{$\S$ A.5. Extrapolation}

Lemme A.5.1. Soit F la fonction analytique associée au polynôme construit précédemment. Alors, pour tout couple $(\underline{t}, s) \in \mathbb{N}^{d+1}$ avec $|\underline{t}| \leq 2(d+1) T$ et $s \leq 2(d+1) S$, l'inégalité suivante est vérifiée :

$$
\begin{aligned}
\left|\frac{1}{\underline{t} !} \mathcal{D}_{\mathbf{f}}^{\underline{t}} F(s \mathbf{u})-\frac{1}{\underline{t} !} \mathcal{D}_{\mathbf{f}}^{\underline{t}} F(s \mathbf{w})\right| \leq & c_{53}^{T+\digamma+\log S} \times|\Lambda| \times \max _{\underline{\lambda}}\left\{\left|p_{\underline{\lambda}}\right|\right\} \times\left(1+S\left\|\mathbf{u}_{0}\right\|\right)^{D_{0}} \\
& \times \exp \left\{c_{53} \sum_{j=1}^{n} D_{j}\left(1+S\left\|\mathbf{u}_{j}\right\|\right)^{\rho_{j}}\right\} .
\end{aligned}
$$

La démonstration de ce lemme découle du lemme I.12.2, de l'hypothèse A.1.3 (qui permet de majorer le nombre de $\underline{\lambda}$ par $\left.e^{c_{54} T}\right)$ et de l'

Hypothèse A.5.2. $S|\Lambda| \leq 1$.

Cependant, pour faciliter les calculs, nous supposerons (même si ce n'est pas essentiel) que l'hypothèse suivante (plus forte) est vérifiée :

Hypothèse A.5.3. $S E|\Lambda| \leq 1$. 
Proposition A.5.4 (Extrapolation). Il existe une constante $c_{55}$, ne dépendant que de $(\mathbf{G}, \Phi)$, telle que, pour tout réel $E \geq 1$, pour tout couple $(\underline{t}, s) \in \mathbb{N}^{d+1}$ avec $|\underline{t}| \leq(d+1) T$ et $s \leq(d+1) S$, la quantité

$$
\left|\frac{1}{\underline{t} !} \mathcal{D} \underline{\underline{f}} F(s \mathbf{u})\right|
$$

soit majorée par

1. Dans le cas non-périodique:

$$
\begin{aligned}
& c_{55}^{T+\log S} \times \max _{\underline{\lambda}}\left\{\left|p_{\underline{\lambda}}\right|\right\} \times\left(1+S\left\|\mathbf{u}_{0}\right\|\right)^{D_{0}} \times e^{c_{55} \sum_{j=1}^{n} D_{j}\left(1+S\left\|\mathbf{u}_{j}\right\|\right)^{\rho_{j}}} \\
& \times\left\{\left(1+E S\left\|\mathbf{u}_{0}\right\|\right)^{D_{0}} e^{c_{55} \sum_{j=1}^{n} D_{j}\left(1+E S\left\|\mathbf{u}_{j}\right\|\right)^{\rho_{j}}}\left(\frac{1}{E}\right)^{(d+1) T S_{0}}+\left(\frac{c_{55} S}{S_{0}}\right)^{(d+1) T S_{0}}(1+\|\mathbf{u}\|)^{2(d+1) T}\left(e^{-p}+|\Lambda|\right)\right\}
\end{aligned}
$$

2. Dans le cas périodique:

$$
\begin{aligned}
& c_{55}^{T+T \log (1+\|\mathbf{u}\|)+\log S} \times \max _{\underline{\lambda}}\left\{\left|p_{\underline{\lambda}}\right|\right\} \times\left(1+S\left\|\mathbf{u}_{0}\right\|\right)^{D_{0}} \times e^{c_{55} \sum_{j=1}^{n} D_{j}\left(1+S\left\|\mathbf{u}_{j}\right\|\right)^{\rho_{j}}} \\
& \times\left\{\left(1+E S\left\|\mathbf{u}_{0}\right\|\right)^{D_{0}} e^{c_{55} \sum_{j=1}^{n} D_{j}\left(1+E S\left\|\mathbf{u}_{j}\right\|\right)^{\rho_{j}}}\left(\frac{1}{E}\right)^{(d+1) T_{0} S}+c_{55}^{T_{0} S}\left(e^{-p}+|\Lambda|\right)\right\}
\end{aligned}
$$

En revenant à la base $\widetilde{\mathbf{e}}$ de $W$, nous en déduisons le

Corollaire A.5.5. Pour tous $|\underline{t}| \leq(d+1) T$ et $s \leq(d+1) S$, on a

1. Dans le cas non-périodique:

$$
\begin{aligned}
\left|\underline{\frac{1}{t} !} \mathcal{D}_{\widetilde{\mathbf{e}}}^{\underline{t}} F(s \mathbf{u})\right| \leq & c_{55}^{T+\log S} \times \max _{\underline{\lambda}}\left\{\left|p_{\underline{\lambda}}\right|\right\} \times\left(1+S\left\|\mathbf{u}_{0}\right\|\right)^{D_{0}} \times e^{c_{55} \sum_{j=1}^{n} D_{j}\left(1+S\left\|\mathbf{u}_{j}\right\|\right)^{\rho_{j}}} \\
\times & \left\{\left(1+E S\left\|\mathbf{u}_{0}\right\|\right)^{D_{0}} e^{c_{55} \sum_{j=1}^{n} D_{j}\left(1+E S\left\|\mathbf{u}_{j}\right\|\right)^{\rho_{j}}}\left(\frac{1}{E}\right)^{(d+1) T S_{0}}\right. \\
& \left.+\left(\frac{c_{55} S}{S_{0}}\right)^{(d+1) T S_{0}}(1+\|\mathbf{u}\|)^{2(d+1) T}\left[e^{-p}+|\Lambda|\right]\right\}
\end{aligned}
$$

2. Dans le cas périodique:

$$
\begin{aligned}
\left|\frac{1}{\underline{t} !} \mathcal{D}_{\widetilde{\mathbf{e}}}^{t} F(s \mathbf{u})\right| \leq & c_{55}^{T \log (e+\|\mathbf{u}\|)+\log S} \times \max _{\underline{\underline{\lambda}}}\left\{\left|p_{\underline{\lambda}}\right|\right\} \times\left(1+S\left\|\mathbf{u}_{0}\right\|\right)^{D_{0}} \times e^{c_{55} \sum_{j=1}^{n} D_{j}\left(1+S\left\|\mathbf{u}_{j}\right\|\right)^{\rho_{j}}} \\
& \times\left\{\left(1+E S\left\|\mathbf{u}_{0}\right\|\right)^{D_{0}} e^{c_{55} \sum_{j=1}^{n} D_{j}\left(1+E S\left\|\mathbf{u}_{j}\right\|\right)^{\rho_{j}}}\left(\frac{1}{E}\right)^{(d+1) T_{0} S}\right. \\
& \left.+c_{55}^{T_{0} S}\left[e^{-p}+|\Lambda|\right]\right\}
\end{aligned}
$$

\section{$\S$ A.6. Estimations ultramétriques et archimédiennes}

Les lemmes I.14.5 et I.15.1 sont inchangés. 


\section{$\S$ A.7. Conclusion}

Rappelons qu'à partir de maintenant le couple $(m, \underline{t})$ est celui fixé après le lemme I.14.3. Des estimations ultramétriques et archimédiennes, nous déduisons alors (formule du produit) la minoration :

$$
\begin{aligned}
& \frac{1}{D} \log \left|\frac{1}{\prod_{j=0}^{n}\left(\varphi_{\tau_{j}}^{(j)}\left(m \mathbf{u}_{j}\right)\right)^{D_{j}}} \frac{1}{\underline{t} !} \mathcal{D}_{\underline{\underline{\mathbf{e}}}}^{\underline{t}} F(m \mathbf{u})\right| \\
& \geq-c_{56} T-D_{0} h(W)-\frac{1}{D} \sum_{v \neq \sigma_{0}} \log \max _{\underline{\underline{\lambda}}}\left\{\left|p_{\underline{\lambda}}\right|_{v}\right\}-(d+1) T \log D_{0} \\
& \quad+\sum_{h=1}^{n} \frac{D_{h}}{D} \sum_{v \neq \sigma_{0}} \log \left|A_{\tau_{h}}^{(h)}\left(\psi_{0}^{(h)}(0), \psi_{\tau_{h}}^{(h)}\left(m \mathbf{u}_{h}\right)\right)\right|_{v}-\frac{D_{0}}{D} \sum_{v \neq \sigma_{0}} \log \max \left\{1,\left|m u_{0}\right|_{v}\right\} \\
& \quad-\sum_{h=1}^{n} \mathbf{c}_{h} \frac{D_{h}}{D} \sum_{v \neq \sigma_{0}} \log \max \left\{\left|\frac{\varphi_{0}^{(h)}\left(m \mathbf{u}_{h}\right)}{\varphi_{\tau_{h}}^{(h)}\left(m \mathbf{u}_{h}\right)}\right|_{v}, \cdots,\left|\frac{\varphi_{N_{h}}^{(h)}\left(m \mathbf{u}_{h}\right)}{\varphi_{\tau_{h}}^{(h)}\left(m \mathbf{u}_{h}\right)}\right|_{v}\right\}
\end{aligned}
$$

puis

$$
\begin{aligned}
& \frac{1}{D} \log \left|\frac{1}{\underline{t} !} \mathcal{D}_{\widetilde{\mathbf{e}}}^{\underline{t}} F(m \mathbf{u})\right| \\
& \geq-c_{57} \sum_{h=1}^{n} D_{h}\left(\max _{0 \leq s \leq(d+1) S}\left\{h\left(s \mathbf{p}_{h}\right)\right\}+\frac{1}{D}\left(1+S\left\|\mathbf{u}_{h}\right\|\right)^{\rho_{h}}\right)-(d+1) T \log D_{0} \\
& \quad-D_{0}\left(h(W)+\frac{1}{D} \sum_{v \neq \sigma_{0}} \log \max \left\{1,\left|m u_{0}\right|_{v}\right\}\right)-\frac{1}{D} \sum_{v \neq \sigma_{0}} \log \max _{\underline{\lambda}}\left\{\left|p_{\underline{\lambda}}\right|_{v}\right\}
\end{aligned}
$$

après avoir remarqué, toujours en utilisant la formule du produit, que

$$
\begin{aligned}
\frac{1}{D} \sum_{v \neq \sigma_{0}} \log \left|A_{\tau_{h}}^{(h)}\left(\psi_{0}^{(h)}(0), \psi_{\tau_{h}}^{(h)}\left(m \mathbf{u}_{h}\right)\right)\right|_{v} & =-\frac{1}{D} \log \left|A_{\tau_{h}}^{(h)}\left(\psi_{0}^{(h)}(0), \psi_{\tau_{h}}^{(h)}\left(m \mathbf{u}_{h}\right)\right)\right| \\
& \geq-\frac{\mathbf{c}_{h}}{D} \log \max \left\{\left|\frac{\varphi_{0}^{(h)}\left(m \mathbf{u}_{h}\right)}{\varphi_{\tau_{h}}^{(h)}\left(m \mathbf{u}_{h}\right)}\right|, \cdots,\left|\frac{\varphi_{N_{h}}^{(h)}\left(m \mathbf{u}_{h}\right)}{\varphi_{\tau_{h}}^{(h)}\left(m \mathbf{u}_{h}\right)}\right|\right\}-\frac{c_{58}}{D}
\end{aligned}
$$

En comparant la minoration (A.7) à la majoration du corollaire A.5.5 et en remplaçant la hauteur de $P$ qui apparaît par son majorant (page 48), nous obtenons l'existence d'une constante $c_{59}$ vérifiant : 
(1) Dans le cas non-périodique, la quantité

$$
\begin{aligned}
¥_{1}:= & \frac{p}{C_{0} D}+h\left(\xi_{1}: \cdots: \xi_{D}\right)+T \log D_{0}+\log D \\
& +\sum_{h=1}^{n} D_{h}\left(\max _{0 \leq s \leq(d+1) S}\left\{h\left(s \mathbf{p}_{h}\right)\right\}+\frac{\left(S\left\|\mathbf{u}_{h}\right\|\right)^{\rho_{h}}}{D}\right)+D_{0}\left(h(W)+\max _{0 \leq s \leq(d+1) S}\left\{h\left(s u_{0}\right)\right\}\right) \\
& +\frac{1}{c_{59} D} \log \left\{\left(\frac{1}{E}\right)^{(d+1) T S_{0}}\left(1+E S\left|u_{0}\right|\right)^{D_{0}} e^{c_{59} \sum_{h=1}^{n} D_{h}\left(1+E S\left\|\mathbf{u}_{h}\right\|\right)^{\rho_{h}}}\right. \\
& \left.+\left(\frac{c_{59} S}{S_{0}}\right)^{(d+1) T S_{0}}(1+\|\mathbf{u}\|)^{2(d+1) T}\left[e^{-p}+|\Lambda|\right]\right\}
\end{aligned}
$$

est positive. Supposons alors que $e^{-p}+|\Lambda|$ est inférieur ou égal à

$$
\left(\frac{S_{0}}{c_{59} S}\right)^{(d+1) T S_{0}} \frac{1}{(1+\|\mathbf{u}\|)^{2(d+1) T}}\left(\frac{1}{E}\right)^{(d+1) T S_{0}}\left(1+E S\left|u_{0}\right|\right)^{D_{0}} e^{c_{59} \sum_{h=1}^{n} D_{h}\left(1+E S\left\|\mathbf{u}_{h}\right\|\right)^{\rho_{h}}}
$$

et choisissons

$$
\begin{aligned}
p:=C_{0}^{1 / 2} T S_{0} \log E-C_{0} D h\left(\xi_{1}: \cdots: \xi_{D}\right)-C_{0} D T \log D_{0}-C_{0} D \log D \\
\quad-C_{0} \sum_{h=1}^{n} D_{h}\left(\max _{0 \leq s \leq(d+1) S}\left\{D h\left(s \mathbf{p}_{h}\right)\right\}+\left(E S\left\|\mathbf{u}_{h}\right\|\right)^{\rho_{h}}\right) \\
\quad-C_{0} D_{0}\left(D h(W)+\max _{0 \leq s \leq(d+1) S}\left\{D h\left(s u_{0}\right)\right\}+\log \left(1+E S\left|u_{0}\right|\right)\right)
\end{aligned}
$$

Alors $¥_{1}$ est négative ce qui contredit l’hypothèse sur $e^{-p}+|\Lambda|$ et conduit à une minoration de $|\Lambda|$.

(2) Dans le cas périodique (et compte tenu de la minoration de $\mathrm{d}\left(\mathbf{u}, t_{\widetilde{\mathbf{G}}}(\mathbb{C})\right) \mathrm{du}$ corollaire A.2.3), la quantité

$$
\begin{aligned}
¥_{2}:= & T\left(\log D_{0}+\frac{1}{D} \log (1+\|\mathbf{u}\|)\right)+h\left(\xi_{1}: \cdots: \xi_{D}\right)+\log D \\
& +\frac{p}{C_{0} D}+D_{0}\left(h(W)+\max _{0 \leq s \leq(d+1) S}\left\{h\left(s u_{0}\right)\right\}\right)+\sum_{h=1}^{n} D_{h}\left(\max _{0 \leq s \leq(d+1) S}\left\{h\left(s \mathbf{p}_{h}\right)\right\}+\frac{\left(S\left\|\mathbf{u}_{h}\right\|\right)^{\rho_{h}}}{D}\right) \\
& +\frac{1}{c_{59} D} \log \left\{\left(\frac{1}{E}\right)^{(d+1) T_{0} S}\left(1+E S\left|u_{0}\right|\right)^{D_{0}} e^{c_{59} \sum_{h=1}^{n} D_{h}\left(1+E S\left\|\mathbf{u}_{h}\right\|\right)^{\rho_{h}}}\right. \\
& \left.+c_{59}^{T_{0} S}\left[e^{-p}+|\Lambda|\right]\right\}
\end{aligned}
$$

est positive. Supposons que $e^{-p}+|\Lambda|$ est inférieur ou égal à

$$
\left(\frac{1}{c_{59} E}\right)^{(d+1) T_{0} S}\left(1+E S\left|u_{0}\right|\right)^{D_{0}} e^{c_{59} \sum_{h=1}^{n} D_{h}\left(1+E S\left\|\mathbf{u}_{h}\right\|\right)^{\rho_{h}}}
$$


et choisissons

$$
\begin{aligned}
p:=C_{0}^{1 / 2} & T_{0} S \log E-C_{0} D h\left(\xi_{1}: \cdots: \xi_{D}\right)-C_{0} D \log D \\
& -C_{0} D T\left(\log D_{0}+\frac{1}{D} \log (1+\|\mathbf{u}\|)\right) \\
& -C_{0} \sum_{h=1}^{n} D_{h}\left(\max _{0 \leq s \leq(d+1) S}\left\{D h\left(s \mathbf{p}_{h}\right)\right\}+\left(E S\left\|\mathbf{u}_{h}\right\|\right)^{\rho_{h}}\right) \\
& -C_{0} D_{0}\left(D h(W)+\max _{0 \leq s \leq(d+1) S}\left\{D h\left(s u_{0}\right)\right\}+\log \left(1+E S\left|u_{0}\right|\right)\right)
\end{aligned}
$$

Alors $¥_{2}$ est négative et on obtient encore une minoration de $|\Lambda|$.

En résumé, nous obtenons le

\section{Résultat intermédiaire}

Avec les données du $\S$ I.2 de la première partie (le groupe algébrique, etc ...), il existe une constante $c_{60}$ ne dépendant que de $(\mathbf{G}, \Phi)$ telle que : pour tous réels strictement positifs $\widetilde{T}_{0}<\widetilde{T}, S_{0}<S, \widetilde{D}_{0}, \ldots, \widetilde{D}_{n}, x_{0}, C_{0}, p, E$ vérifiant les conditions suivantes :

1. $T^{\#}=x_{0} \widetilde{T}, T=\left[T^{\#}\right]$.

2. $E \geq 1$

3. $x_{0} \geq \frac{(\widetilde{T})^{d} \operatorname{card}\left(\Gamma_{\mathbf{p}}(S)\right)}{C_{0}\left(\operatorname{deg}_{\Phi} \mathbf{G}\right) \widetilde{D}_{0}^{\delta_{0}} \ldots \widetilde{D}_{n}^{\delta_{n}}}$

4. $\widetilde{T}_{0} \geq c_{60}\left(\max \left\{\widetilde{D}_{0}, \ldots, \widetilde{D}_{n}\right\}+\frac{2}{x_{0}}\right)$

5. $C_{0} \geq c_{60}$

6. $S E|\Lambda| \leq 1$

Alors :

$\star$ Ou bien $\mathbf{u} \notin W$ et

1. Dans le cas non-périodique :

$$
\begin{aligned}
|\Lambda| \geq\left(\frac{S_{0}}{c_{60} S}\right)^{(d+1) T S_{0}} \frac{1}{(1+\|\mathbf{u}\|)^{2(d+1) T}}\left(\frac{1}{E}\right)^{(d+1) T S_{0}} \\
-\exp \left\{-C_{0}^{1 / 2} T S_{0} \log E+C_{0} D h\left(\xi_{1}: \cdots: \xi_{D}\right)+C_{0} D \log D\right. \\
+C_{0} D T \log \left(x_{0} \widetilde{D}_{0}\right)+C_{0} x_{0} \sum_{h=1}^{n} \widetilde{D}_{h}\left(\max _{0 \leq s \leq(d+1) S}\left\{D h\left(s \mathbf{p}_{h}\right)\right\}+\left(E S\left\|\mathbf{u}_{h}\right\|\right)^{\rho_{h}}\right) \\
\left.+C_{0} x_{0} \widetilde{D}_{0}\left(D h(W)+\max _{0 \leq s \leq(d+1) S}\left\{D h\left(s u_{0}\right)\right\}+\log \left(1+E S\left|u_{0}\right|\right)\right)\right\}
\end{aligned}
$$


2. Dans le cas périodique :

$$
\begin{aligned}
|\Lambda| \geq\left(\frac{1}{c_{61} E}\right)^{(d+1) T_{0} S} & \\
-\exp \{ & -C_{0}^{1 / 2} T_{0} S \log E+C_{0} D h\left(\xi_{1}: \cdots: \xi_{D}\right)+C_{0} D \log D \\
& +C_{0} x_{0} \widetilde{D}_{0}\left(D h(W)+\max _{0 \leq s \leq(d+1) S}\left\{D h\left(s u_{0}\right)\right\}+\log \left(1+E S\left|u_{0}\right|\right)\right) \\
& +C_{0} x_{0} \sum_{h=1}^{n} \widetilde{D}_{h}\left(\max _{0 \leq s \leq(d+1) S}\left\{D h\left(s \mathbf{p}_{h}\right)\right\}+\left(E S\left\|\mathbf{u}_{h}\right\|\right)^{\rho_{h}}\right) \\
& \left.+C_{0} D T\left(\log \left(x_{0} \widetilde{D}_{0}\right)+\frac{1}{D} \log (1+\|\mathbf{u}\|)\right)\right\}
\end{aligned}
$$

$\star$ Ou bien $\mathbf{u} \in W$ et alors il existe un sous-groupe algébrique connexe $\widetilde{\mathbf{G}}$ de $\mathbf{G}$ tel que

$$
\operatorname{deg}_{\Phi} \pi(\widetilde{\mathbf{G}}) \leq\left(\operatorname{deg}_{\Phi} \mathbf{G}\right) C_{0} \times \frac{x_{0} \widetilde{D}_{0}}{\operatorname{card}\left(\frac{\Gamma_{\mathbf{p}}(S)+\widetilde{\mathbf{G}}(\mathbf{L})}{\widetilde{\mathbf{G}}(\mathbf{L})}\right)} \times \max \left\{\left(\frac{\widetilde{D}_{j_{1}}}{\widetilde{T}}\right) \cdots\left(\frac{\widetilde{D}_{j_{\tilde{r}-1}}}{\widetilde{T}}\right)\right\}
$$

(cf. lemme A.2.1) et $\mathbf{u} \in t_{\widetilde{\mathbf{G}}}(\mathbb{C}) \subseteq W \otimes \mathbb{C}$.

Sous cette forme extrêmement technique, le résultat ci-dessus n'est guère exploitable. Tout au plus, il permet de s'assurer que le choix des paramètres effectué page 18 est valide, sans aucune certitude vis-à-vis de sa pertinence. Alors, pour ne pas laisser le lecteur sur sa faim, nous allons démontrer que l'on ne peut espérer une amélioration sensible de la mesure d'indépendance (en particulier pour la hauteur du point $\mathbf{p}$ ) avec des manipulations élémentaires sur les paramètres sans idées nouvelles.

\section{$\S$ A.8. Épilogue ...}

... opératique! Pour mieux comprendre l'influence de chaque paramètre sur la minoration finale de $\mathrm{d}(\mathbf{u}, W)$, nous allons décrire les « groupes »de paramètres qui apparaissent ensemble, dresser un tableau des contraintes puis donner la mesure obtenue. In fine, nous montrerons que le choix des paramètres effectué au $\S$ I.7 est le meilleur possible, au regard des contraintes énoncées.

\section{$\S$ A.8.a. Les protagonistes}

(1) Le Chef d'Orchestre :

$$
\frac{1}{D} T S \log E
$$

Ce terme apparaît dans le lemme d'extrapolation (type Schwarz) *. Il est au cœur de la partie analytique de la méthode de Baker. Le facteur $1 / D$ signifie que la majoration fine n'est effectuée que pour une seule place de $\mathbf{L}$ (celle correspondant au plongement initial de $\mathbf{L}$ dans $\mathbb{C}$ ). C'est lui qui décide de la qualité de la mesure d'indépendance.

*Pour être précis, c'est $(1 / D) T S_{0} \log E$ qui apparaît dans le cas non-périodique et $(1 / D) T_{0} S \log E$ dans le cas périodique. 
(2) La Prima Donna :

$$
T \cdot \log D_{0}
$$

Cette quantité est au centre même de la thèse que vous lisez. Elle est de nature arithmétique ; elle mesure la taille des dénominateurs des coefficients de Taylor de la fonction auxiliaire (lemme I.14.5, page 38). Grâce à un modèle lisse de $\mathbf{G}$ et à son logarithme formel, elle remplace la grandeur $T \cdot \log T$, source de l'apparition du logarithme de $h(W)$, qui intervenait dans les travaux antérieurs [45,65]. Signalons que $T \log D_{0}$ apparait aussi indépendamment dans le cas périodique via l'inégalité de changements de bases (25) (page 27) et la majoration (A.4), page 47.

(3) Le Ténor :

$$
D_{0} \cdot h(W)
$$

L'existence de ce terme découle de l'astuce de N. HiRATA-KoHNo, qui modifie l'hyperplan pour le positionner transversalement à l'espace tangent $t_{\mathbb{G}_{\mathrm{a}}}$. Il remplace le terme $T \cdot h(W)$ qui apparaissait dans les travaux antérieurs à [45], et qui provoquait la venue (au moins) de $h(W)^{2}$ dans la mesure d'indépendance. Il joue un rôle essentiel et va de pair avec la quantité précédente.

(4) Les Barytons:

$$
D_{0} \cdot\left(\max _{0 \leq s \leq(d+1) S}\left\{h\left(s u_{0}\right)\right\}+\frac{1}{D} \log \left(1+E S\left|u_{0}\right|\right)\right)
$$

Ce terme comprend les quantitées liées à $\mathbb{G}_{\mathrm{a}}$ et découle de la nature polynomiale des fonctions sur $\mathbb{G}_{\mathrm{a}}$ (d'où le logarithme!).

$$
D_{h} \cdot\left(\max _{0 \leq s \leq(d+1) S}\left\{h\left(s \mathbf{p}_{h}\right)\right\}+\frac{1}{D}\left(E S\left\|\mathbf{u}_{h}\right\|\right)^{\rho_{h}}\right)
$$

Ce terme est également classique et regroupe, à l'instar de son confrère, l'aspect arithmétique et analytique (via le logarithme $\mathbf{u}_{h}$ ) du point $\mathbf{p}_{h} \in \mathbf{G}_{h}(\overline{\mathbb{Q}})$.

(5) Les seconds rôles :

$$
\frac{T}{D} \cdot \log (1+\|\mathbf{u}\|)
$$

Dans le cas non-périodique, cette quantité apparaît en utilisant la formule de dérivation (37), page 35. Dans le cas périodique, c'est le choix de la base $\mathbf{f}$ et la comparaison avec la base $\widetilde{\mathbf{e}}$ qui provoque son entrée en scène (inégalité (26) de la proposition I.10.2, page 27).

$$
h\left(\xi_{1}: \cdots: \xi_{D}\right)
$$

Ce terme est relatif au choix de la base du corps de nombres L. C'est la construction du polynôme auxiliaire, via le lemme de Thue-Siegel, qui justifie sa présence. L'utilisation de la méthode des pentes supprime ce terme. Il est majoré par $D\left(h(W)+h\left(\mathbf{p}_{0}\right)+\cdots+\right.$ $\left.h\left(\mathbf{p}_{n}\right)\right)+c_{62}$.

(6) Les figurants :

$$
T, S, D_{0}, \ldots, D_{n}
$$

Ils sont presque toujours en duo avec des constantes ne dépendant que de $(\mathbf{G}, \Phi)$. 


\section{$\S$ A.8.b. Tableau des contraintes}

Pour $a \in \mathbb{R}$, la notation $a \gg 1$ signifie que $a$ est supérieur à une constante ne dépendant que de $(\mathbf{G}, \Phi)$.

Voici les contraintes (écrites dans le cas non-périodique ; dans le cas périodique, il suffit, dans la toute suite, de remplacer $T_{0}$ par $T$ et $S$ par $S_{0}$; en particulier, la condition (A.16) ci-dessous est supprimée) :

$$
\frac{1}{D} T S_{0} \log E \gg \sqrt{C_{0}} D_{h}\left(\max _{0 \leq s \leq(d+1) S}\left\{h\left(s \mathbf{p}_{h}\right)\right\}+\frac{1}{D}\left(E S\left\|\mathbf{u}_{h}\right\|\right)^{\rho_{h}}\right) \quad \text { pour } h=0, \ldots, n
$$

$$
\frac{1}{D} T S_{0} \log E \gg \sqrt{C_{0}} D_{0}\left(h(W)+\max _{0 \leq s \leq(d+1) S}\left\{h\left(s u_{0}\right)\right\}+\frac{1}{D} \log \left(1+E S\left|u_{0}\right|\right)\right)
$$

$$
\frac{1}{D} T S_{0} \log E \gg \sqrt{C_{0}}\left(h\left(\xi_{1}: \cdots: \xi_{D}\right)+\log D\right)
$$

$$
S_{0} \log E \gg \sqrt{C_{0}} D \log D_{0}
$$

$$
S_{0} \log E \gg \frac{1}{\sqrt{C_{0}}} \log (1+\|\mathbf{u}\|)
$$

$$
\sqrt{C_{0}} \log E \gg \log \left(S / S_{0}\right)
$$

$$
T_{0} \gg D_{h} \quad \text { pour } h=0, \ldots, n
$$

$$
\frac{1}{C_{0}^{2}} \geq \max \left\{\frac{1+S_{0}}{1+S}, \frac{\widetilde{T}_{0}}{\widetilde{T}}\right\}
$$

$$
C_{0} \gg 1
$$

$$
x_{0} \geq \frac{\widetilde{T}^{d} \operatorname{card}\left(\Gamma_{\mathbf{p}}(S)\right)}{C_{0} \times \operatorname{deg}_{\Phi} \mathbf{G} \times \widetilde{D}_{0}^{\delta_{0}} \cdots \widetilde{D}_{n}^{\delta_{n}}} .
$$

Cette dernière condition est de type « condition de Siegel $»$ (nombre d'équations/nombre d'inconnues) :

$$
T^{d} S \simeq C_{0} D_{0}^{\delta_{0}} \cdots D_{n}^{\delta_{n}}
$$

Sous toutes ces conditions, la mesure d'indépendance linéaire est alors

$$
\log |\Lambda| \gg-\sqrt{C_{0}} \max \left\{T S_{0}, T_{0} S\right\} \log E .
$$

* Cette condition n'intervient que dans le cas périodique. Dans l'autre cas, il suffit de remplacer dans la conclusion (inégalité (A.21)) la quantité $S_{0} \log E$ par $S_{0} \log E+\log (1+\|\mathbf{u}\|)$. 


\section{$\S$ A.8.c. Justification du choix des paramètres}

Pour éviter la répétition (avec d'infimes variantes), nous allons étudier seulement le cas non-périodique.

Nous avons à résoudre un problème d'optimisation sous contraintes .

Un point de vue heuristique suggère, d'une part, que «la » solution du problème réside au bord du sous-ensemble des points

$$
\left(x_{0}, \widetilde{T}, \widetilde{T}_{0}, S, S_{0}, \widetilde{D}_{0}, \ldots, \widetilde{D}_{n}\right)
$$

de $\mathbb{R}^{n+6}$ qui satisfont aux contraintes du $\S$ A.8.b et, d'autre part, que la (relative) symétrie qui existe entre les groupes $\mathbf{G}_{1}, \ldots, \mathbf{G}_{n}$ doit être conservée (un des $D_{\ell}(1 \leq \ell \leq n)$ ne peut être fonction de $h(W)$ sans que les autres $D_{j}(j \neq \ell, 1 \leq j \leq n)$ ne soient, eux aussi, fonction de $\left.h(W)\right)$. Nous allons voir que ces considérations simples sont ici confirmées.

D'après les conditions (A.11) et (A.17), pour $1 \leq h \leq n$, le réel $\widetilde{D}_{h}$ doit être le quotient de $\widetilde{T} S_{0} \log E$ par

$$
\sqrt{C_{0}}\left(\max _{0 \leq s \leq(d+1) S}\left\{D h\left(s \mathbf{p}_{h}\right)\right\}+\left(E S\left\|\mathbf{u}_{h}\right\|\right)^{\rho_{h}}\right)+S_{0} \log E,
$$

pondéré par un coefficient $\left.\varepsilon_{h} \in\right] 0,1[$. De même,

$$
\widetilde{D}_{0}=\frac{\widetilde{T} S_{0} \log E}{\sqrt{C_{0}}\left(D h(W)+\max _{0 \leq s \leq(d+1) S}\left\{D h\left(s u_{0}\right)\right\}+\log \left(1+E S\left|u_{0}\right|\right)\right)+S_{0} \log E} \times \varepsilon_{0},
$$

avec $\left.\varepsilon_{0} \in\right] 0,1[$. De plus, les paramètres doivent vérifier la condition de Siegel (condition (3) du théorème A.7, ou inégalité (A.20)), i.e.

$$
\begin{aligned}
x_{0} \geq & \frac{(\widetilde{T})^{d} \cdot \operatorname{card}\left(\Gamma_{\mathbf{p}}(S)\right)}{C_{0} \widetilde{D}_{0}^{\delta_{0}} \cdots \widetilde{D}_{n}^{\delta_{n}}} \\
\geq & \frac{C_{0}^{d} \cdot \operatorname{card}\left(\Gamma_{\mathbf{p}}(S)\right)}{\left(S_{0} \log E\right)^{d+1} \widetilde{T}} \times\left(\sqrt{C_{0}}\left(D h(W)+\max _{0 \leq s \leq(d+1) S}\left\{D h\left(s u_{0}\right)\right\}+\log \left(1+E S\left|u_{0}\right|\right)\right)+S_{0} \log E\right) \\
& \times \prod_{j=1}^{n}\left(\sqrt{C_{0}}\left\{\max _{0 \leq s \leq(d+1) S}\left\{D h\left(s \mathbf{p}_{j}\right)\right\}+\left(E S\left\|\mathbf{u}_{j}\right\|\right)^{\rho_{j}}\right\}+S_{0} \log E\right)^{\delta_{j}} \times \frac{1}{\varepsilon_{0}^{\delta_{0}} \cdots \varepsilon_{n}^{\delta_{n}}}
\end{aligned}
$$

(en fait, nous pouvons enlever le terme $\log \left(1+E S\left|u_{0}\right|\right)$ qui est majoré par $D h\left(S u_{0}\right)+\log E$ ) donc, avec l'inégalité (A.21), on retrouve (au mieux) :

$$
\begin{aligned}
\log \mathrm{d}(\mathbf{u}, W) \gg- & \frac{\operatorname{card}\left(\Gamma_{\mathbf{p}}(S)\right)}{\left(S_{0} \log E\right)^{d}} \times\left(D h(W)+\max _{0 \leq s \leq(d+1) S}\left\{D h\left(s u_{0}\right)\right\}+S_{0} \log E\right) \\
& \times \prod_{j=1}^{n}\left(\max _{0 \leq s \leq(d+1) S}\left\{D h\left(s \mathbf{p}_{j}\right)\right\}+\left(E S\left\|\mathbf{u}_{j}\right\|\right)^{\rho_{j}}+S_{0} \log E\right)^{\delta_{j}} \\
& \times \frac{1}{\varepsilon_{0}^{\delta_{0}} \cdots \varepsilon_{n}^{\delta_{n}}} .
\end{aligned}
$$

Il devient clair que la mesure se bonifie en choisissant $\varepsilon_{0}=\cdots=\varepsilon_{n}=1$ (i.e. $D_{0}, \ldots, D_{n}$ maximaux), $S$ minimal (i.e. $C_{0}$ constant et $S=c_{63} \times S_{0}$, avec $c_{63} \geq C_{0}$, cette contrainte étant liée à la majoration du rang du système linéaire (lemme I.11.1, page 28)). Enfin, le choix du nombre de pas* $S_{0}$ est guidé (voire imposé !) par les contraintes (A.14) et (A.15).

${ }^{*}$ Nombre qui, d'après l'inégalité (A.22), doit être choisi le plus petit possible. 


\section{Annexe A. Paramètres libres}

Remarque : un calcul semblable montre que la majoration du degré de $\pi(\widetilde{\mathbf{G}})$ est, elle aussi, optimale (modulo les contraintes énoncées au paragraphe précédent).

Ayant la meilleure mesure possible (à la constante (liée au groupe) près) avec la méthode utilisée, nous allons, dans la seconde partie de cette thèse, changer notre fusil d'épaule en mettant en œuvre la méthode des pentes de J.-B. BosT, qui apporte un point de vue plus géométrique de la situation et un formalisme efficace pour, d'une part, calculer la constante dépendant de $\mathbf{G}$ (lorsque ce groupe est une variété abélienne) et, d'autre part, pour introduire de nouveaux outils, d'usage courant en Théorie d'Arakelov. 


\section{Mesure d'indépendance linéaire de logarithmes II}





\section{Le cas abélien non-homogène}

\section{$\S 1$. Introduction}

L'objet de ce texte est d'obtenir une mesure d'indépendance linéaire de logarithmes de $\mathbf{G}(\overline{\mathbb{Q}})$, où $\mathbf{G}$ est le produit direct du groupe affine $\mathbb{G}_{\mathrm{a}}$ et d'une variété abélienne (définie sur $\overline{\mathbb{Q}})$, entièrement effective, via la méthode des pentes de J.-B. Bost et des outils de Géométrie d'Arakelov qui lui sont naturellement associés. Plus que le résultat lui-même (qui, cependant, est inédit), nous essaierons de mettre en relief la démarche, qui diffère en plusieurs points de celle employée dans la première partie de cette thèse. Un des points saillants de la méthode est l'absence du lemme de Siegel (ou une de ses variantes) car nous n'effectuons aucun choix de polynômes (auxiliaires) mais nous considérons plutôt tous les polynômes, à l'instar de la méthode des déterminants d'interpolation de M. LAURENT. À la base, ces deux méthodes considèrent un même objet mathématique, écrit sous deux aspects différents : une matrice $M$ à coefficients algébriques pour l'une, une application linéaire $\varphi$ entre des $\overline{\mathbb{Q}}$-espaces vectoriels (de dimension finie) pour l'autre. Et elles ont en commun l'évaluation de la puissance extérieur maximale (i.e. le déterminant) de ces objets pour chacune des normes associées aux places (ultramétriques et archimédiennes) de $\overline{\mathbb{Q}}$. La filtration qui intervient dans la méthode des pentes se traduit alors par des manipulations sur les lignes et les colonnes de la matrice $M$ (ce qui ne modifie pas son déterminant). Si, à l'origine, les points de vue sont quasiidentiques*, la méthode des pentes jouit, à mon avis, d'une plus grande souplesse d'utilisation parce qu'aucune base des espaces vectoriels qui entrent dans le définition de $\varphi$ n'est choisie ; cet aspect intrinsèque (contrairement aux déterminants d'interpolation) ouvre la porte à la géométrie (et donc à l'imagination ${ }^{\dagger}$ !) si nous considérons pour ces espaces vectoriels des espaces de sections de fibrés sur un schéma. La géométrie d'Arakelov entre en scène car, aux places infinies, ces espaces vectoriels doivent être munis de métriques, et un moyen efficace de les obtenir (sans avoir à choisir de bases et de décréter qu'elles sont orthonormées) est de disposer d'une structure de fibrés hermitiens, dont se déduisent alors les métriques voulues sur les espaces de sections. Dans le cadre des variétés abéliennes, les objets (arakeloviens)

*À tel point d'ailleurs que l'on retrouve les mêmes difficultés techniques (aujourd'hui non résolues) qui m'obligeront à supposer $u_{0} \neq 0$ (forme linéaire non-homogène), alors qu'une telle hypothèse n'est pas utile avec les « polynômes auxiliaires ». Autrement dit, je ne sais pas « extrapoler sur les dérivées » avec la méthode des pentes mais seulement sur les points (méthode de Baker, stricto sensu).

${ }^{\dagger}$ La méthode des pentes a permis de découvrir de nouveaux énoncés (et, bien sûr, de les démontrer) comme, par exemple, le très joli résultat de [40] ou le «théorème scandaleux » de J.-B. Bost, scandaleux parce qu'il n'a été observé que récemment (2000), dont l'énoncé est le suivant : Soit X une sous-variété analytique (algébrique) lisse de $\mathbb{P}^{n}(\mathbb{C})$, de dimension $\geq 1$, et $\mathscr{X}$ un germe le long de $X$ de sous-variété analytique lisse de $\mathbb{P}^{n}(\mathbb{C})$. Si le fibré normal $N_{X} \mathscr{X}$ est ample sur $X$ alors $\mathscr{X}$ est algébrique. 
introduits dans la méthode des pentes sont calculables de manière totalement explicites. C'est pourquoi nous avons écrit ce texte avec une variété abélienne (et non un groupe algébrique commutatif quelconque), ce qui nous permet d'utiliser les travaux de J.-B. Bost [17, 18, 19, 20, 21] et P. Graftieaux [39, 40]. Le théorème II.2.1 est (dans un cadre plus restreint) une version totalement explicite des théorèmes I.3.1 et I.3.2 de la première partie de cette thèse. D'un point de vue pratique, il est extrêmement agréable de pouvoir utiliser ces résultats (qui masquent parfois des calculs difficiles, voir iii) du théorème II.3.2) de manière immédiate et transparente, même si, parfois, il est possible d'effectuer « à la main » les calculs, à l'instar, par exemple, des travaux récents de S. DAVID et P. PHILIPPON sur les minorations de hauteurs normalisées [32, 33]. Ces formules simplifient grandement les démonstrations mais elles ne sont pas, en elle-mêmes, des arguments cruciaux.

La méthode des pentes est encore peu répandue en Transcendance, en dépit des cours donnés par J.-B. Bost, à l'Institut Henri Poincaré, en 1997 et 1999 [20] et des quelques articles qui utilisent cette méthode [19, 22, 39, 40]. Ainsi, par soucis pédagogique, nous avons repris en annexe l'aspect élémentaire de cette méthode que nous illustrons (très simplement) avec le théorème des six exponentielles. Le lecteur intéressé par d'autres applications (que celles déjà citées) de la méthode des pentes pourra également consulter les thèses de H. RANDRIAMBOLOLONA [67] et E. ViadA-AEHLE [78].

\section{$\S 2$. Données et résultats}

Soit $(\mathbf{A}, L)$ une variété abélienne polarisée (le fibré en droites $L$ est ample et symétrique sur $\mathbf{A})$, définie sur $\overline{\mathbb{Q}}$, de dimension $d$. Notons $\mathrm{h}^{0}(\mathbf{A}, L)$ la dimension de l'espace des sections $\Gamma(\mathbf{A}, L)$ et $h_{F}(\mathbf{A})$ la hauteur de Faltings de $\mathbf{A}$. Notons $\mathbf{G}$ le groupe algébrique (sur $\left.\overline{\mathbb{Q}}\right) \mathbb{G}_{\mathrm{a}} \times \mathbf{A}$ et $t_{\mathbf{G}}(\overline{\mathbb{Q}})$ (identifié à $t_{\mathbb{G}_{a}}(\overline{\mathbb{Q}}) \oplus t_{\mathbf{A}}(\overline{\mathbb{Q}})$ ) l'espace tangent à l'origine de $\mathbf{G}$. Soient exp l'exponentielle du groupe de Lie complexe $\mathbf{G}(\mathbb{C})$ et $\mathbf{u}$ un vecteur de $t_{\mathbf{G}}(\mathbb{C})$ tel que $\mathbf{p}:=\exp (\mathbf{u})$ appartienne à $\mathbf{G}(\overline{\mathbb{Q}})$. Considérons $W$ un hyperplan de $t_{\mathbf{G}}(\overline{\mathbb{Q}})$, ne contenant pas $t_{\mathbb{G}_{\mathbf{a}}}(\overline{\mathbb{Q}})$. Dans ce texte, afin d'éviter un écueil technique*, nous supposerons que la projection de $\mathbf{u}$ sur $t_{\mathbb{G}_{\mathrm{a}}}(\mathbb{C})$ est non nulle (les formes linéaires que nous étudions sont donc non-homogènes ). D'après le théorème de Wüstholz [85] (voir aussi points (a) et (b) du théorème I.3.1), cela implique que $\mathbf{u} \notin W$. Fixons un corps de nombres $k$ sur lequel existe un modèle de Moret-Bailly de $(\mathbf{A}, L,\{\mathbf{p}\}$ ) (voir $\S$ II.3.a pour une définition précise d'un tel modèle. Une construction du corps $k$ est expliquée au début de la démonstration du theorem 4.10 de [18], page 58). Soient $K$ une extension finie de $k$ sur laquelle est définie l'hyperplan $W$ et $D$ un majorant de $[K: \mathbb{Q}]$. Nous supposerons que le corps $K$ est plongé dans $\mathbb{C}$ (nous noterons parfois $\sigma_{0}$ ce plongement particulier). Signalons que, contrairement à la première partie, le corps $k$ n'est pas seulement lié à la variété abélienne mais aussi au choix du point p. Il est cependant indépendant de l'hyperplan $W$. Dorénavant, le groupe $\mathbf{G}$ sera vu comme schéma en groupes au-dessus de Spec $k$. En particulier, l'espace tangent $t_{\mathbf{G}}$ est un $k$-espace vectoriel. Soit $e_{0}\left(=\frac{\partial}{\partial X}\right)$ la base canonique de $t_{\mathbb{G}_{\mathbf{a}}}$ et $\left(e_{1}, \ldots, e_{d}\right)$ une base de $t_{\mathbf{A}}$ (orthonormée pour le produit scalaire sur $t_{\mathbf{A}_{\sigma_{0}}}(\mathbb{C}$ ), considéré quelques lignes plus loin). La famille $\left\{e_{0}, \ldots, e_{d}\right\}$ forme une base de $t_{\mathbf{G}}$, dans laquelle les coordonnées de $\mathbf{u}$

\footnotetext{
*Actuellement, nous ne savons pas traiter le cas périodique (voir définition I.7.2, p. 21) avec la méthode des pentes ou, le problème est le même, avec la méthode des déterminants d'interpolations de M. LAURENT (voir note $*$ précédente).
} 
sont $\left(u_{0}, \ldots, u_{d}\right)$ et $W$ a pour équation $z_{0}=\beta_{1} z_{1}+\cdots+\beta_{d} z_{d}$, avec $\beta_{i} \in K$ (c'est possible car $\left.t_{\mathbb{G}_{\mathrm{a}}} \not \subset W\right)$. Notons $\mathbf{q} \in \mathbf{A}(k)$ la projection de $\mathbf{p}$ sur $\mathbf{A}(k)$, et $\mathbf{u}^{\prime}$ un logarithme $\left(\in t_{\mathbf{A}_{\sigma_{0}}}(\mathbb{C})\right)$ de $\mathbf{q}$. Notons également $\widehat{h}(\mathbf{q})$ la hauteur de Néron-Tate (relative à $L$ ) de q. Pour chaque plongement $\sigma: K \hookrightarrow \mathbb{C}$, nous disposons d'une norme hermitienne $\|\cdot\|_{L, \sigma}$ sur $t_{\mathbf{G}_{\sigma}}(\mathbb{C})$, de distance associée $\mathrm{d}_{\sigma}$, définie au $\S$ II.3.b.. Soit $\mathscr{A}$ un schéma au dessus de $\mathcal{O}_{k}$, de fibre générique A, provenant du modèle de Moret-Bailly, considéré ci-dessus. Une définition précise de $\mathscr{A}$ se situe au début du $\S$ II.5.a. .

Le théorème suivant porte sur une minoration de la distance de $\mathbf{u}$ à $W$ (relativement au plongement fixé $\sigma_{0}: K \hookrightarrow \mathbb{C}$ ), distance qui est aussi égale au module du nombre complexe

$$
\Lambda:=u_{0}-\beta_{1} u_{1}-\cdots-\beta_{d} u_{d}
$$

divisé $\operatorname{par}\left(1+\left|\beta_{1}\right|^{2}+\cdots+\left|\beta_{d}\right|^{2}\right)^{1 / 2}$.

Théorème II.2.1. Supposons les hypothèses ci-dessus vérifiées. Supposons de plus que le fibré ample $L$ induise une polarisation principale sur A. Soit $E$ un réel $\geq$ e. Soient a et $b$ des réels vérifiant

$$
\begin{gathered}
\log a \geq \max \left\{1, \widehat{h}(\mathbf{q}), \frac{E^{2}\left\|\mathbf{u}^{\prime}\right\|_{L, \sigma_{0}}^{2}}{D}\right\}, \\
\log b \geq h\left(u_{0}\right)+\frac{1}{[K: \mathbb{Q}]} \sum_{\sigma: K \hookrightarrow \mathbb{C}} \log \frac{1}{\mathrm{~d}_{\sigma}\left(e_{0}, W \otimes_{\sigma(K)} \mathbb{C}\right)} .
\end{gathered}
$$

Alors

$$
\begin{aligned}
\log \mathrm{d}_{\sigma_{0}}(\mathbf{u}, W) \geq & -(100 d)^{1000 d^{2}}\left\{1+\frac{D}{\log E}\left(\max \left\{1, h_{F}(\mathbf{A})\right\}+\log ^{+}\left(\frac{D \log a}{\log E}\right)\right)\right\}^{d+1} \\
& \times\left\{D \log b+\log E+D \log \left(1+\frac{D}{\log E}\left(\max \left\{1, h_{F}(\mathbf{A})\right\}+\log ^{+}\left(\frac{D \log a}{\log E}\right)\right)\right)\right\} \\
& \times\left\{1+\frac{D \log a}{\log E}\right\}^{d} .
\end{aligned}
$$

Remarque : $\mathrm{Si}(\mathbf{A}, L)$ n'est pas principalement polarisé, nous pouvons, par isogénie, nous ramener aux conditions de ce théorème et l'inégalité (1) reste vraie en remplaçant

$$
\max \left\{1, h_{F}(\mathbf{A})\right\}
$$

par

$$
\max \left\{1, h_{F}(\mathbf{A}), \log \mathrm{h}^{0}(\mathbf{A}, L)\right\} .
$$

À ma connaissance, cette inégalité et le résultat de S. DAVID [30], qui traitait le cas où la variété abélienne est un produit de courbes elliptiques, sont les seules mesures d'indépendances linéaires totalement explicites, sur un groupe algébrique (commutatif) non exclusivement linéaire. Cependant, notre résultat, s'il est du même type que [30], ne le contient pas. En effet, d'une part, nous avons supposé $u_{0} \neq 0$ et, d'autre part, nous avons considéré la variété abélienne polarisée $(\mathbf{A}, L)$ dans sa globalité. La méthode de démonstration du théorème II.2.1 
permet de traiter* le cas où $\mathbf{A}$ est le produit (d'un nombre fini) de variétés abéliennes polarisées $\left(\mathbf{A}_{i}, L_{i}\right)$, en tenant compte séparément de chacun des invariants $\left(h_{F}\left(\mathbf{A}_{i}\right), \mathrm{h}^{0}\left(\mathbf{A}_{i}, L_{i}\right)\right)$ liés à $\left(\mathbf{A}_{i}, L_{i}\right)$. Nous allons voir que la méthode des pentes et quelques théorèmes de géométrie d'Arakelov simplifient à la fois la démonstration et les calculs (il en reste !). Auparavant, afin de mieux percevoir les conséquences de ce théorème, nous allons donner une suite de mesures d'indépendances linéaires, chacune d'elles étant centrée sur un paramètre précis et se déduisant aisément du théorème II.2.1.

Conséquence II.2.2. Il existe une constante $\mathrm{c}_{1}=\mathrm{c}_{1}(\mathbf{A}, L, K, \mathbf{u})>0$, indépendante de la hauteur $h(W)$ de $W$, telle que

$$
\log |\Lambda| \geq-\mathrm{c}_{1} \max \{1, h(W)\} .
$$

Ce résultat est vrai dans le cadre général des groupes algébriques commutatifs (voir le théorème principal de la première partie). Il est optimal, à la constante $c_{1}$ près. De plus, la conséquence II.2.2 se déduit du théorème II.2.1 en comparant la quantité

$$
h^{\prime}(W):=\frac{1}{[K: \mathbb{Q}]} \sum_{\sigma: K \hookrightarrow \mathbb{C}} \log \frac{1}{\mathrm{~d}_{\sigma}\left(e_{0}, W \otimes_{\sigma(K)} \mathbb{C}\right)}
$$

à $h(W)$.

Pour cela, fixons une place $\sigma: K \hookrightarrow \mathbb{C}$ et notons $(,)_{\sigma}$ le produit scalaire sur $t_{\mathbf{G}_{\sigma}}(\mathbb{C})$ qui orthonormalise la base $\left(e_{0}, \ldots, e_{d}\right)$. La distance $\mathrm{d}_{\sigma}^{\prime}$ induite par $(,)_{\sigma}$ et la distance $\mathrm{d}_{\sigma}$ sont équivalentes, donc il existe des constantes $\mathrm{c}_{1, \sigma}, \mathrm{c}_{2, \sigma}>0$ (indépendantes de $W$ ) telles que

$$
\mathrm{c}_{2, \sigma} \mathrm{d}_{\sigma}^{\prime}\left(e_{0}, W_{\sigma}\right) \geq \mathrm{d}_{\sigma}\left(e_{0}, W_{\sigma}\right) \geq \mathrm{c}_{1, \sigma} \mathrm{d}_{\sigma}^{\prime}\left(e_{0}, W_{\sigma}\right)
$$

où $W_{\sigma}:=W \otimes_{\sigma(K)} \mathbb{C}$. Nous appliquons alors la formule de Gram à $\left(t_{\mathbf{G}_{\sigma}}(\mathbb{C}), \mathrm{d}_{\sigma}^{\prime}\right)$ et nous trouvons

$$
\mathrm{d}_{\sigma}^{\prime 2}\left(e_{0}, W_{\sigma}\right)=\frac{\operatorname{det}\left(\widetilde{e}_{i}, \widetilde{e}_{j}\right)_{0 \leq i, j \leq d}}{\operatorname{det}\left(\widetilde{e}_{i}, \widetilde{e}_{j}\right)_{1 \leq i, j \leq d}}
$$

où $\widetilde{e}_{i}:=e_{i}+\sigma\left(\beta_{i}\right) e_{0}$ pour $i=1, \ldots, d$ et $\widetilde{e}_{0}:=e_{0}$. Un calcul de déterminant donne alors

$$
\mathrm{d}_{\sigma}^{\prime}\left(e_{0}, W_{\sigma}\right)=\frac{1}{\left(1+\left|\sigma\left(\beta_{1}\right)\right|^{2}+\cdots+\left|\sigma\left(\beta_{d}\right)\right|^{2}\right)^{1 / 2}}
$$

et donc

$$
h(W)+\mathrm{c}^{\prime} \leq h^{\prime}(W) \leq h(W)+\mathrm{c}
$$

où $c:=\frac{1}{[K: \mathbb{Q}]} \sum_{\sigma} \log \frac{\sqrt{d+1}}{c_{1, \sigma}}$ et $c^{\prime}:=\frac{1}{[K: \mathbb{Q}]} \sum_{\sigma} \log \frac{1}{c_{2, \sigma}}$.

Conséquence II.2.3. Il existe une constante $\mathrm{c}_{2}=\mathrm{c}_{2}(\mathbf{A}, L, K,\|\mathbf{u}\|, W)>0$, indépendante de la hauteur (de Néron-Tate) de $\mathbf{q}$ et de la hauteur (de Weil) de $u_{0}$, telle que

$$
\log |\Lambda| \geq-\mathrm{c}_{2}\left(h\left(u_{0}\right)+\log \max \{e, \widehat{h}(\mathbf{q})\}\right)\left(\frac{\max \{e, \widehat{h}(\mathbf{q})\}}{\log \max \{e, \widehat{h}(\mathbf{q})\}}\right)^{d} .
$$

*Avec seulement quelques calculs supplémentaires! 
Pour démontrer cette inégalité, nous utilisons le théorème II.2.1 avec $E=\max \left\{e, \widehat{h}(\mathbf{q})^{1 / 2}\right\}$.

Nous pouvons aussi analyser la « qualité »de la mesure (1) vis-à-vis des paramètres secondaires $D, h_{F}(\mathbf{A})$ et $\mathrm{h}^{0}(\mathbf{A}, L)$, en comparant avec les mesures antérieures de S. David [30] et N. Hirata-Kohno [45]. Ainsi, en choisissant $E=\sqrt{D}$, le membre de droite de la mesure (1) est de l'ordre de

$$
\frac{D^{2 d+2}}{(\log (1+D))^{d-1}}
$$

quantité inchangée par rapport à [30, 45]. De même, signalons que la dépendance en la hauteur de Faltings $h_{F}(\mathbf{A})$ de $\mathbf{A}$ de la mesure (1) est légèrement plus faible que celle de [30]. Le rapport des deux mesures (pour ce paramètre) est (après renormalisation) de l'ordre de

$$
\frac{\max \left\{1, h_{F}(\mathbf{A})\right\}}{\left(\log \max \left\{e, h_{F}(\mathbf{A})\right\}\right)^{d+2}} .
$$

De manière plus anecdotique, à l'aide de la remarque qui suit le théorème II.2.1, nous pouvons regarder la dépendance du membre de droite de (1) en $\mathrm{h}^{0}(\mathbf{A}, L)$. Cela donne

$$
\frac{\mathrm{h}^{0}(\mathbf{A}, L)^{d}}{\left(\log \left(1+\mathrm{h}^{0}(\mathbf{A}, L)\right)\right)^{d-1}},
$$

en oubliant toutes les données sauf $\mathrm{h}^{0}(\mathbf{A}, L)$. De ce point de vue, l'inégalité (1) est totalement inédite ; en effet, une courbe elliptique est toujours principalement polarisée et dans ce cas la dimension $h^{0}(\mathbf{A}, L)$ vaut 1 . C'est pourquoi le résultat de S. DAVID [30] ne comprenait pas cet invariant.

Remarque : l'expression (1) explicite la dépendance en le fibré ample $L$, qui apparaît à trois endroits :

i) la hauteur $\widehat{h}(\mathbf{q})=\widehat{h}_{L}(\mathbf{q})$.

ii) la dimension $\mathrm{h}^{0}(\mathbf{A}, L)$.

iii) le terme $h^{\prime}(W)$ du minorant de $\log b$.

Le passage de $L$ à $L^{\otimes n}\left(n \in \mathbb{N}^{*}\right)$ transforme $\widehat{h}(\mathbf{q})$ en $n \widehat{h}(\mathbf{q}), \mathrm{h}^{0}(\mathbf{A}, L)$ en $n^{d} \mathrm{~h}^{0}(\mathbf{A}, L)$ mais $\mathrm{d}_{\sigma_{0}}(\mathbf{u}, W)$ en $n \mathrm{~d}_{\sigma_{0}}(\mathbf{u}, W)$. Ainsi il n'y a qu'un facteur $\log n$ en plus, tandis que le minorant augmenterait, lui, polynomialement. En l'état actuel de la minoration, je ne vois pas d'espoir pour que le passage de $L$ à $L^{\otimes n}$ puisse, en faisant tendre $n$ vers l'infini, éliminer les « termes en trop » de la mesure d'indépendance, même si je disposais d'une telle mesure sur le produit de variétés abéliennes.

Avant de démontrer le théorème II.2.1 (§ II.5), nous procéderons à quelques rappels concernant les métriques usuelles que l'on peut attacher aux objets géométriques (fibré en droites, espace tangent) que nous rencontrerons.

Dans le cadre de notre étude, la partie arithmétique de la démonstration utilise la technique de changement de variables de CHUDNOvsky. Le point de vue schématique introduit une variante dans l'utilisation de cette technique, en évitant le recours aux formules d'additions (« explicites ») sur la variété abélienne. Quant à la partie analytique, elle repose, comme dans la première partie, sur la méthode de Baker.

\section{$\S 3$. Boîte à outils}

Chacune des parties qui composent cette section peut être lue indépendamment du reste du texte. 


\section{§ 3.a. Modèles de Moret-Bailly}

La définition qui suit donne toutes les propriétés d'un «bon » modèle arakelovien de $(\mathbf{A}, L)$ dont nous aurons besoin dans la suite. Le théorème de ce paragraphe nous assure de l'existence de ce modèle, ainsi que du bon comportement de la hauteur d'un point par rapport à un faisceau inversible de fibre générique $L$. Pour plus de détails, le lecteur pourra se reporter au $\S 4.3$ de [18] (dont est extrait ce qui suit).

Définition II.3.1. Soient $A$ une variété abélienne de dimension $g$, définie sur un corps de nombres $k_{0}$ et $L$ un fibré en droites ample et symétrique sur $A$. Soit $\mathcal{F}$ un ensemble de points de $A\left(k_{0}\right)$. Un MB-modèle de $(A, L, \mathcal{F})$ au-dessus de $k_{0}$ est la donnée des objets suivants :

1. un schéma en groupe (lisse et) semi-stable $\pi: \mathcal{A} \rightarrow \operatorname{Spec} \mathcal{O}_{k_{0}}$

2. un isomorphisme $\gamma: A \rightarrow \mathcal{A}_{\overline{\mathbb{Q}}}$

3. un fibré en droite hermitien cubiste $\overline{\mathcal{L}}$ sur $\mathcal{A}$

4. un isomorphisme $\psi: L \rightarrow \gamma^{*} \mathcal{L}_{\overline{\mathbb{Q}}}$ de fibrés inversibles sur $A$

5. pour tout $H \in \mathcal{F}$, une section $\varepsilon_{H}: \operatorname{Spec} \mathcal{O}_{k_{0}} \rightarrow \mathcal{A}$ de $\pi$ dont la restriction à la fibre générique est égal à $\gamma(H)$

qui satisfont à la propriété suivante : il existe un sous-schéma $\mathcal{K}$ de $\mathcal{A}$, plat et fini sur Spec $\mathcal{O}_{k_{0}}$, tel que $\gamma^{-1}\left(\mathcal{K}_{\overline{\mathbb{Q}}}\right)$ coincide avec le groupe de Mumford $K\left(L^{\otimes 2}\right)$.

Le théorème suivant assure l'existence d'un MB-modèle d'une variété abélienne polarisée et il apporte le calcul du degré d'Arakelov normalisé (noté $\widehat{\operatorname{deg}}_{n}$ ) de certains fibrés hermitiens obtenus à partir de ce MB-modèle.

Théorème II.3.2. Soient $A$ une variété abélienne de dimension g, définie sur un corps de nombres $k_{0}$ et $L$ un fibré en droites ample et symétrique sur $A$. Soit également $\mathcal{F}$ un ensemble de points de $A\left(k_{0}\right)$. Alors

i) Il existe une extension finie $k$ de $k_{0}$ et un $M B$-modèle

$$
\left(\pi: \mathcal{A} \rightarrow \operatorname{Spec} \mathcal{O}_{k}, i, \overline{\mathcal{L}}, \psi,\left(\varepsilon_{P}\right)_{P \in \mathcal{F}}\right)
$$

$\operatorname{pour}(A, L, \mathcal{F})$ au-dessus de $k$.

ii) En notant $\widehat{h}(P)$ la hauteur de Néron-Tate (relative à $L)$ de $P(\in \mathcal{F})$, on a l'égalité : $\widehat{h}(P)=\widehat{\operatorname{deg}}_{\mathrm{n}} \frac{\varepsilon_{P}^{*} \mathcal{L}}{}$.

iii) De plus $\widehat{\operatorname{deg}_{\mathrm{n}}} \overline{\pi_{*} \mathcal{L}}=\mathrm{h}^{0}(A, L)\left(-\frac{1}{2} h_{F}(A)+\frac{1}{4} \log \frac{\mathrm{h}^{0}(A, L)}{(2 \pi)^{g}}\right)$ où $h_{F}(A)$ est la hauteur de Faltings de $A$ et $\mathrm{h}^{0}(A, L)$ la dimension de l'espace des sections $L(A)$.

Ce théorème revêt une importance particulière ici. L'existence d'un modèle de MoretBailly pour la variété abélienne polarisée $(\mathbf{A}, L)$ ne fournit pas seulement un modèle lisse de $A$ mais un modèle qui de surcroît permet de calculer les constantes liées à $(\mathbf{A}, L)$. Le calcul du degré d'Arakelov des sections globales de $\mathcal{L}$ (partie iii) du théorème) repose sur le théorème de Riemann-Roch arithmétique de H. Gillet et C. Soulé [37] ainsi que sur les travaux de L. Moret-BAilly [60, 61] (voir également [16]). 


\section{$\S$ 3.b. Métriques}

Dans ce paragraphe, nous décrivons les métriques attachées aux différents objets géométriques que nous considérerons dans la démonstration du théorème II.2.1.

\section{Métrique de Fubini-Study}

Les notes qui suivent proviennent essentiellement de [23, 41, 74].

Soit $n$ un entier naturel non nul. On note $\mathbb{P}^{n}(\mathbb{C})=\operatorname{Proj}\left(\mathbb{C}\left[X_{0}, \ldots, X_{n}\right]\right)$ l'espace projectif complexe usuel et $\mathcal{O}(1)$ le fibré canonique sur $\mathbb{P}^{n}(\mathbb{C})$. On rappelle le

Lemme II.3.3. Soit $X$ une variété analytique complexe et $\bar{L}=(L,\|\|$.$) un fibré hermitien$ sur $X$. Alors il existe une unique $(1,1)$-forme différentielle $c_{1}(\bar{L})$ telle que, pour tout ouvert $U$ de $X$ et toute section (holomorphe) s de L au-dessus de $U$, qui ne s'annule pas sur $U$ (i.e. qui induit une trivialisation de $L(U)$ ), alors

$$
c_{1}(\bar{L})_{\mid U}=\frac{1}{2 i \pi} \partial \bar{\partial} \log \|s\|^{2} .
$$

La construction de $c_{1}(\bar{L})$ — appelée forme de Chern du fibré hermitien $\bar{L}$ - à l'aide d'un recouvrement de $X$ et des fonctions de transitions de $L$ associées se trouve (par exemple) au $\S 2.8$ du premier chapitre de [74].

Définition II.3.4. La 2 -forme différentielle (de type $(1,1)$ ) sur $\mathbb{P}^{n}$

$$
\omega=-\frac{1}{2 i \pi} \partial \bar{\partial} \log \|z\|^{2}
$$

où $\|z\|^{2}=\left|z_{0}\right|^{2}+\ldots+\left|z_{n}\right|^{2}$ est dite forme de Fubini-Study.

La forme $\omega$ est fermée, invariante sous l'action de $U_{n+1}(\mathbb{C})$ et définie positive. Notons $d=\partial+\bar{\partial}$ et $d^{c}=\frac{\partial-\bar{\partial}}{4 i \pi}$ les opérateurs classiques. Alors on a localement

$$
\begin{aligned}
\omega & =d d^{c} \log |z|^{2} \\
& =\frac{d d^{c}|z|^{2}}{|z|^{2}}-\frac{d|z|^{2} \wedge d^{c}|z|^{2}}{|z|^{4}} \\
& =\frac{i}{2 \pi}\left(\frac{1}{|z|^{2}} \sum_{i=0}^{n} d z_{i} \wedge d \bar{z}_{i}-\frac{1}{|z|^{4}} \sum_{i=0}^{n} \bar{z}_{i} d z_{i} \wedge z_{i} d \bar{z}_{i}\right) .
\end{aligned}
$$

La métrique de Fubini-Study $\left(\operatorname{sur} \mathcal{O}_{\mathbb{P}_{\mathbb{C}}^{n}}(1)\right)$ est la métrique définie ainsi : on munit $\mathbb{C}^{n+1}$ de la métrique hermitienne standard, ce qui induit une métrique sur $\mathcal{O}^{n+1}=\mathcal{O} . X_{0} \oplus \mathcal{O} . X_{1} \oplus$ $\ldots \oplus \mathcal{O} \cdot X_{n}$ et donc par restriction sur le fibré de Hopf $\mathcal{O}_{\mathbb{P}_{\mathbb{C}}^{n}}(-1)$ et par dualité sur $\mathcal{O}_{\mathbb{P}_{\mathbb{C}}^{n}}(1)$. Le forme $\omega$ de Fubini-Study est alors la forme de courbure du fibré en droites hermitien $\overline{\mathcal{O}_{\mathbb{P}_{\mathbb{C}}^{n}}(1)}$. Le volume de l'espace projectif pour cette métrique est $\int_{\mathbb{P}^{n}(\mathbb{C})} \omega^{n}=1$.

Par produit tensoriel, les fibrés $\mathcal{O}(d):=\mathcal{O}(1)^{\otimes d}(d \in \mathbb{N})$ sont munis de métriques que l'on peut explicitement écrire sous la forme suivante : soit $s$ une section globale de $\mathcal{O}(d)$. Cette section s'identifie ( via un isomorphisme naturel) à un polynôme homogène de degré $d$

$$
P\left(X_{0}, \ldots, X_{n}\right)=\sum_{|\underline{\alpha}|=d} p_{\underline{\alpha}} \cdot X_{0}^{\alpha_{0}} \cdots X_{n}^{\alpha_{n}} .
$$


Alors, si $x=\left(x_{0}, \ldots, x_{n}\right) \in \mathbb{P}^{n}(\mathbb{C})$, on a

$$
\|s(x)\|=\frac{\left|P\left(x_{0}, x_{1}, \ldots, x_{n}\right)\right|}{\left(\sum_{i=0}^{n}\left|x_{i}\right|^{2}\right)^{d / 2}} .
$$

De plus si on note

$$
\|s\|_{L^{2}}^{2}=\int_{\mathbb{P}^{n}(\mathbb{C})}\|s(x)\|^{2} \omega
$$

on a

$$
\|s\|_{L^{2}}^{2}=\sum_{|\underline{\alpha}|=d}\left|p_{\underline{\alpha}}\right|^{2} \cdot \frac{n ! \alpha_{0} ! \cdots \alpha_{n} !}{(n+d) !} .
$$

Ces formules s'étendent aux sections multihomogènes : soient $n_{1}, \ldots, n_{k}$ des entiers naturels et $\mathcal{O}\left(d_{1}, \ldots, d_{k}\right)=\mathcal{O}\left(d_{1}\right) \otimes \cdots \otimes \mathcal{O}\left(d_{k}\right)$ le fibré, obtenu par produit externe sur la variété projective produit $\mathbb{P}(\mathbb{C}):=\mathbb{P}^{n_{1}}(\mathbb{C}) \times \cdots \times \mathbb{P}^{n_{k}}(\mathbb{C})$. Les sections globales de ce fibré correspondent aux polynômes en $\left(n_{1}+1\right)+\cdots+\left(n_{k}+1\right)$ variables, multihomogènes de multidegré $\left(d_{1}, \ldots, d_{k}\right)$. En munissant $\mathcal{O}\left(d_{1}, \ldots, d_{k}\right)$ de la métrique induite par celle sur $\mathcal{O}(1)$, en prenant une section globale $s$ de $\mathcal{O}\left(d_{1}, \ldots, d_{k}\right)$ de polynôme $P$ associé, et si $x=\left(\left(x_{0, i}, x_{1, i} \ldots, x_{n_{i}, i}\right)\right)_{i \in\{1, \ldots, k\}} \in$ $\mathbb{P}(\mathbb{C})$, alors on a

$$
\|s(x)\|=\frac{\left|P\left(x_{0,1}, \ldots, x_{n_{k}, k}\right)\right|}{\prod_{i=1}^{k}\left(\sum_{h=0}^{n_{i}}\left|x_{h, i}\right|^{2}\right)^{d_{i} / 2}}
$$

et en notant

$$
P=\sum_{\left|\underline{\alpha}_{i}\right|=d_{i}} p_{\underline{\alpha}_{1}, \ldots, \underline{\alpha}_{k}} \cdot \prod_{j=1}^{k} X_{0, j}^{\alpha_{0, j}} \cdots X_{n_{j}, j}^{\alpha_{n_{j}, j}}
$$

on a

$$
\|s\|_{\mathrm{L}^{2}}^{2}=\sum_{\left|\underline{\alpha}_{i}\right|=d_{i}}\left|p_{\underline{\alpha}_{1}, \ldots, \underline{\alpha}_{k}}\right|^{2} \cdot \prod_{j=1}^{k} \frac{n_{j} ! \alpha_{0, j} ! \cdots \alpha_{n_{j}, j} !}{\left(n_{j}+d_{j}\right) !} .
$$

C'est ce type de formule qui permet dans la pratique de calculer la norme d'une section en un point. L'inégalité de Cauchy-Schwarz implique l'inégalité de Gromov ${ }^{*}$ suivante :

Lemme II.3.5. Si $s \in \Gamma\left(\mathbb{P}, \mathcal{O}_{\mathbb{P}}\left(d_{1}, \ldots, d_{k}\right)\right)$ alors

$$
\|s\|_{\infty} \leq\|s\|_{\mathrm{L}^{2}} \times \prod_{i=1}^{k}\left(\begin{array}{c}
n_{i}+d_{i} \\
n_{i}
\end{array}\right)^{1 / 2}
$$

ò̀ $\|s\|_{\infty}:=\sup _{x \in \mathbb{P}(\mathbb{C})}\|s(x)\|$.

À titre d'information, signalons l'existence d'une inégalité plus générale, qui induit des comparaisons entre la norme $\mathrm{L}^{\infty}$ et la norme $\mathrm{L}^{p}$ de $s$ :

*La terminologie 《inégalité de Gromov » désigne la comparaison entre la norme sup et la norme $\mathrm{L}^{2} \mathrm{~d}$ 'une section d'un fibré hermitien sur une variété complexe compacte. L'archétype d'une telle inégalité se trouve au $\S 5.2 .3$ de [37], page 539 . 
Proposition II.3.6 (Proposition 1.4.2 de [23]). Avec les notations précédentes, notons $\mu$ la forme volume normalisée sur $\mathbb{P}(\mathbb{C})$. Alors

$$
\log \|s\|_{\infty} \leq \int_{\mathbb{P}(\mathbb{C})}(\log \|s(x)\|) \mu(x)+\sum_{i=1}^{k} \sum_{j=1}^{n_{i}} \frac{d_{i}}{2 j}
$$

et cette inégalité est optimale.

\section{Métrique sur l'espace tangent $t_{\mathbf{G}_{\sigma}}(\mathbb{C})$}

Chaque plongement $\sigma: k \rightarrow \mathbb{C}$ confère à $\mathbb{C}$ une structure de $k$-espace vectoriel. Par définition, l'espace $t_{\mathbf{G}_{\sigma}}(\mathbb{C})$ est le tensorisé de l'espace tangent à l'origine $t_{\mathbf{G}}$ et du corps $\mathbb{C}$ audessus de $(k, \sigma)$. Au cours de la démonstration du théorème II.2.1 (§ II.5.a.), nous utiliserons le schéma $\mathscr{X}_{k}=\mathbb{P}_{k}^{1} \times \mathbf{A}$ qui possède le même espace tangent que $\mathbf{G}$ (car $\mathbf{G} \hookrightarrow \mathscr{X}_{k}$ est une immersion ouverte donc étale). L'espace vectoriel $t_{\mathbf{G}_{\sigma}}(\mathbb{C})$ est somme directe de $t_{\mathbf{A}_{\sigma}}(\mathbb{C})$ et de $t_{\mathbb{G}_{\mathrm{a}}}(\mathbb{C})=\mathbb{C}$. $e_{0}$, où $e_{0}$ est la base canonique de $t_{\mathbb{G}_{\mathrm{a}}}$. À l'instar de [19], page 118, nous munissons $t_{\mathbf{A}_{\sigma}}(\mathbb{C})$ de la structure hermitienne construite à partir de la première classe de Chern du fibré en droites complexes $L_{\sigma} \rightarrow \mathbf{A}_{\sigma}(\mathbb{C})$. Nous noterons $\|\cdot\|_{L, \sigma}$ cette norme hermitienne (qui dépend de la polarisation $L$, et donc d'un plongement de $\mathbf{A}$ dans un espace projectif) et $\|\cdot\|_{t_{\mathbf{G}_{\sigma}}(\mathbb{C})}$ la métrique hermitienne sur $t_{\mathbf{G}_{\sigma}}(\mathbb{C})$ obtenue par recollement orthogonal de la métrique triviale $\operatorname{sur} t_{\mathbb{G}_{a}}(\mathbb{C})\left(\left\|e_{0}\right\|_{\sigma}=1\right)$ et de $\|\cdot\|_{L, \sigma}$. Enfin, nous désignerons par $\mathrm{d}_{\sigma}$ la distance sur $t_{\mathbf{G}_{\sigma}}(\mathbb{C})$ associée à $\|\cdot\|_{t_{\mathbf{G}_{\sigma}}(\mathbb{C})}$.

\section{Métrique sur la puissance symétrique}

Soient $(E,()$,$) un \mathbb{C}$-espace vectoriel hermitien (de dimension finie) et $n \in \mathbb{N}^{*}$. La forme sesquilinéaire $\Phi_{n}$ sur $E^{\otimes n}$ définie par : $\Phi_{n}\left(x_{1} \otimes \cdots \otimes x_{n}, y_{1} \otimes \cdots \otimes y_{n}\right)=\prod_{i=1}^{n}\left(x_{i}, y_{i}\right)$ confère à $E^{\otimes n}$ une structure d'espace hermitien, qui se transmet, par quotient, à la $n^{i e ̀ m e}$ puissance symétrique $S^{n}(E)$. De manière explicite, si $<\mid>_{n}$ désigne le produit hermitien ainsi obtenu sur $S^{n}(E)$, on a :

$$
<x_{1} \cdots x_{n} \mid y_{1} \cdots y_{n}>_{n}:=\frac{1}{n !} \sum_{\tau \in \mathfrak{S}_{n}} \prod_{i=1}^{n}\left(x_{i}, y_{\tau(i)}\right),
$$

où $\mathfrak{S}_{n}$ est l'ensemble des permutations de $\{1, \ldots, n\}$. En particulier, si $\left(e_{1}, \ldots, e_{r}\right)$ est une base orthonormée de $E$ et $x=e_{1}^{\alpha_{1}} \cdots e_{r}^{\alpha_{r}} \in S^{n}(E)$ (où $\alpha_{1}+\cdots+\alpha_{r}=n$ ) alors la norme $\|x\|_{n}$ de $x$ vaut

$$
\sqrt{\frac{\alpha_{1} ! \cdots \alpha_{r} !}{n !}}
$$

Pour une approche plus systématique, nous renvoyons le lecteur à Bourbaki [24], en prenant garde qu'il ne choisit pas la même convention pour $<\mid>_{n}$ et considère plutôt $n$ ! $<\mid>_{n}$. Ce détail revêt une grande importance ici car en multipliant la métrique sur $S^{n}(E)$ par $n$ ! (comme le fait Bourbaki), nous augmenterions la pente maximale d'un facteur $n \log n$ et, dans l'usage que que nous en ferons, cela reviendrait à réintroduire le logarithme de la hauteur de l'hyperplan. Cette convention sera utilisée lors des estimations archimédiennes de certains coefficients de Taylor (voir $\S$ II.5.f et, en particulier, la formule (39), p. 87). 


\section{Métrique sur un fibré inversible d'une variété abélienne complexe}

Les rappels de ce paragraphe proviennent en grande partie des travaux de Moret-Bailly [59, $60,61]$.

Soient $A$ une variété abélienne définie sur $\mathbb{C}$ et $L$ un fibré en droites sur $A$. Pour $I$ sousensemble de $\{1,2,3\}$ (éventuellement vide), notons $p_{I}: A^{3} \rightarrow A,\left(x_{1}, x_{2}, x_{3}\right) \mapsto \sum_{i \in I} x_{i}$ et $\mathcal{D}_{3}(L)$ le fibré sur $A^{3}$ :

$$
\mathcal{D}_{3}(L)=p_{123}^{*} L \otimes p_{12}^{*} L^{-1} \otimes p_{23}^{*} L^{-1} \otimes p_{13}^{*} L^{-1} \otimes p_{1}^{*} L \otimes p_{2}^{*} L \otimes p_{3}^{*} L .
$$

D'après le théorème du cube, ce fibré est trivial. En fait on dispose [59] du

Théorème II.3.7. Avec les notations ci-dessus, l'ensemble $\pi(A, L)$ des métriques hermitiennes $C^{\infty}$ positives sur $L$ telles que la métrique induite sur $\mathcal{D}_{3}(L)$ soit triviale est non vide. De plus, $\pi(A, L)$ est aussi l'ensemble des métriques dont la forme de courbure $c_{1}(\bar{L})$ est invariante par translation.

Définition II.3.8. Les éléments de $\pi(A, L)$ sont appelés métriques permises, ou métriques du cube.

On peut préciser le théorème de la façon suivante : donnons-nous un isomorphisme $\varphi$ de fibrés entre $\mathcal{D}_{3}(L)$ et $\mathcal{O}_{A^{3}}$ et munissons $\mathcal{O}_{A^{3}}$ de la métrique triviale. Appelons $\|$. $\|_{\varphi}$ la métrique induite sur $\mathcal{D}_{3}(L)$ par l'isomorphisme $\varphi$. On sait que le choix de $\varphi$ détermine le choix d'une rigidification $\kappa$ de $L$ à l'origine. Le fait est qu'il existe une unique métrique $\nu \in \pi(A, L)$ telle que $\mathcal{D}_{3}(\nu)=\|\cdot\|_{\varphi}$. De plus $\nu$ est compatible à la rigidification de $L$, i.e. induit par $\kappa$ une isométrie entre $\varepsilon^{*} L$ et $\mathcal{O}_{A}$. Lorsque la variété abélienne $A$ est définie sur un corps de nombres $K$ et si $L$ est un fibré en droite sur $A$ alors, pour chaque plongement $\sigma: K \hookrightarrow \mathbb{C}$, on dispose du fibré $L_{\sigma}$ sur la variété abélienne complexe $A_{\sigma}(\mathbb{C})$ déduit de $L$ par le changement de base Spec $\mathbb{C} \stackrel{\text { Spec } \sigma}{\longrightarrow} \operatorname{Spec} K$. Le théorème II.3.7 permet donc de munir $L_{\sigma}$ d'une métrique cubiste $\|.\|_{\sigma}$ et $L$ d'une structure de fibrés hermitiens sur $A$. Comme ce sont les seules métriques à courbure invariante par translation, cela implique que si $L$ est un fibré ample, $\sigma: K \rightarrow \mathbb{C}$ est une place de $K$, si $s \in \Gamma\left(A_{\sigma}(\mathbb{C}), L_{\sigma}\right)$ et si $\vartheta$ est la fonction thêta de $t_{A_{\sigma}}(\mathbb{C}) \rightarrow \mathbb{C}$ correspondant à $s$ via le facteur d'automorphie canonique, alors

$$
\left\|s\left(\exp _{A_{\sigma}(\mathbb{C})}(z)\right)\right\|_{\sigma}^{2}=e^{-\pi\|z\|_{L, \sigma}^{2}|\vartheta(z)|^{2}}
$$

où $\|.\|_{L, \sigma}$ est la métrique sur $t_{A_{\sigma}(\mathbb{C})}$ induite par la $(1,1)$ forme de Chern $c_{1}\left(L_{\sigma}\right)$ (voir $\S$ II.3.b.) et

$$
\exp _{A_{\sigma}(\mathbb{C})}: t_{A_{\sigma}}(\mathbb{C}) \rightarrow A_{\sigma}(\mathbb{C})
$$

l'exponentielle complexe du groupe de Lie $A_{\sigma}(\mathbb{C})$. De manière plus précise, le fibré $\exp _{A_{\sigma}}^{*}\left(L_{\sigma}\right)$ sur $t_{A_{\sigma}}(\mathbb{C})$ est trivial et, d'après la théorie des facteurs d'automorphie (appendice de [12]) l'espace des sections globales $\Gamma\left(A_{\sigma}(\mathbb{C}), L_{\sigma}\right)$ est isomorphe à un espace de fonctions thêta, et le choix de la trivialisation de $\exp _{A_{\sigma}}^{*}\left(L_{\sigma}\right)$ détermine le facteur d'automorphie associé à cet espace de fonctions thêta. On choisit le facteur d'automorphie canonique $a_{L_{\sigma}}$ associé $L_{\sigma}$ qui, après le choix d'une donnée d'Appell-Humbert $\left(H_{\sigma}, \chi_{\sigma}\right)$ de $L_{\sigma}\left(H_{\sigma}\right.$ est la forme bilinéaire associée à $\left.\|.\|_{L, \sigma}\right)$, s'écrit, pour $\omega \in \Omega_{A_{\sigma}(\mathbb{C})}$ (réseau des périodes de $A_{\sigma}(\mathbb{C})$ ) et $z \in t_{A_{\sigma}}(\mathbb{C})$ :

$$
a_{L_{\sigma}}(\omega, z)=\chi_{\sigma}(z) e^{\pi H_{\sigma}(\omega, z)+\frac{\pi}{2}\|\omega\|_{L, \sigma}^{2}} .
$$


Ainsi $\Gamma\left(A_{\sigma}(\mathbb{C}), L_{\sigma}\right)$ est isomorphe à

$$
\mathcal{T}_{\sigma}:=\left\{\vartheta: t_{A_{\sigma}}(\mathbb{C}) \rightarrow \mathbb{C} ; \forall(\omega, z) \in \Omega_{A_{\sigma}(\mathbb{C})} \times t_{A_{\sigma}}(\mathbb{C}) \vartheta(\omega+z)=a_{L_{\sigma}}(\omega, z) \vartheta(z)\right\} .
$$

L'espace $\mathcal{T}_{\sigma}$ est muni d'une structure hermitienne ponctuelle ( $c f$. [47], § 4.3, page 33) :

$$
\forall z \in t_{A_{\sigma}}(\mathbb{C}), \forall\left(\vartheta, \vartheta^{\prime}\right) \in \mathcal{T}_{\sigma}, \quad\left(\vartheta, \vartheta^{\prime}\right)_{z}=e^{-\pi\|z\|_{L, \sigma}^{2} \vartheta(z) \overline{\vartheta^{\prime}(z)}} .
$$

Ce nombre complexe ne dépend en fait que de la classe de $z$ modulo $\Omega_{A_{\sigma}(\mathbb{C})}$, ce qui induit une structure hermitienne globale sur $\mathcal{T}_{\sigma}$ :

$$
\|\vartheta\|_{\sigma}^{2}=\int_{t_{A_{\sigma}}(\mathbb{C}) / \Omega_{A_{\sigma}(\mathbb{C})}} e^{-\pi\|z\|_{L, \sigma}^{2}|\vartheta(z)|^{2} d \mu_{\sigma}(z)}
$$

où $\mu_{\sigma}$ est une mesure de Haar sur $t_{A_{\sigma}}(\mathbb{C}) / \Omega_{A_{\sigma}(\mathbb{C})}$. L'isomorphisme entre $\Gamma\left(A_{\sigma}(\mathbb{C}), L_{\sigma}\right)$ et $\mathcal{T}_{\sigma}$ donne alors à $\Gamma\left(A_{\sigma}(\mathbb{C}), L_{\sigma}\right)$ la métrique ponctuelle $\mathrm{L}^{2}$ induite par celle de $\mathcal{T}_{\sigma}$. Comme la forme de courbure de cette métrique est invariante par translation sur $\Omega_{A_{\sigma}(\mathbb{C})}$, elle coïncide par le théorème II.3.7 avec la métrique du cube sur $L_{\sigma}$. On en déduit l'égalité (6).

\section{$\S$ 3.c. Quelques résultats auxiliaires}

Les lemmes, d'intérêt indépendant, énoncés dans ce paragraphe ne sont pas essentiels pour la démonstration du théorème II.2.1. Cependant, ils permettent d'apporter des précisions (semi-stabilité, comparaison de hauteurs) sur certains objets utilisés lors de la preuve.

\section{Irréductibilité d'une action de groupe}

Rappelons que l'action d'un groupe $G$ sur un espace vectoriel $E$ est dite irréductible si les seuls sous-espaces de $E$, stables sous l'action de $G$, sont $\{0\}$ et $E$.

Lemme II.3.9. Soit $\mathfrak{k}$ un corps commutatif algébriquement clos. Soient $E_{1}, E_{2}$ des $\mathfrak{k}$-espaces vectoriels de dimension finie et $G_{1}, G_{2}$ des groupes qui agissent irréductiblement sur (respectivement) $E_{1}$ et $E_{2}$. Alors $G_{1} \times G_{2}$ agit naturellement sur $E_{1} \otimes_{\mathfrak{k}} E_{2}$ et cette action est irréductible.

Démonstration. L'action de $G_{1} \times G_{2}$ sur $E_{1} \otimes_{\mathfrak{k}} E_{2}$ est donnée par

$$
\left(\left(g_{1}, g_{2}\right), \sum_{i} e_{i} \otimes f_{i}\right) \longmapsto \sum_{i} g_{1} . e_{i} \otimes g_{2} . f_{i}
$$

Soit $V$ un sous-espace vectoriel non-nul de $E_{1} \otimes E_{2}$, stable par $G:=G_{1} \times G_{2}$. Définissons l'entier $h$ comme le minimum des $n \in \mathbb{N}^{*}$ pour lesquels il existe $x_{1}, \ldots, x_{n} \in E_{1}$, non tous nuls, et il existe $y_{1}, \ldots, y_{n} \in E_{2}$, $\mathfrak{k}$-linéairement indépendants, tels que $x_{1} \otimes y_{1}+\cdots+x_{n} \otimes y_{n}$ appartienne à $V$ (l'ensemble des entiers $n$ est non vide car $V \neq\{0\})$. Soient $x_{1}, \ldots, x_{h}, y_{1}, \ldots, y_{h}$ vérifiant les conditions précédentes. Soit $V_{1}$ l'ensemble

$$
\left\{x \in E_{1} ; \exists z_{2}, \ldots, z_{h} \in E_{1} ; x \otimes y_{1}+z_{2} \otimes y_{2}+\cdots+z_{h} \otimes y_{h} \in V\right\} .
$$

C'est un sous-espace vectoriel de $E_{1}$, non réduit à $\{0\}$ (il contient $x_{1}$ ) et stable par $G_{1}$. Donc $V_{1}=E_{1}$. De plus, pour chaque $x \neq 0$ élément de $V_{1}$, les $z_{2}, \ldots, z_{h} \in E_{1}$ tels que $x \otimes y_{1}+z_{2} \otimes y_{2}+\cdots+z_{h} \otimes y_{h} \in V$ sont uniques car, sinon, en effectuant la différence, on aurait $\left(z_{2}-z_{2}^{\prime}\right) \otimes y_{2}+\cdots+\left(z_{h}-z_{h}^{\prime}\right) \otimes y_{h} \in V$, ce qui contredirait la minimalité de $h$. Notons $z_{i}=f_{i}(x)$. L'unicité des $z_{i}$ implique que $f_{i} \in \operatorname{End}_{\mathfrak{k}}\left(E_{1}\right)$, pour tout 
$i=2, \ldots, h$. Comme $\mathfrak{k}$ est un corps algébriquement clos, si l'endomorphisme $f_{2}$ est non nul, il possède un vecteur propre $\mathrm{a} \in E_{1} \backslash\{0\}$, de valeur propre associée $\lambda$. Alors $\mathrm{a} \otimes\left(y_{1}+\lambda y_{2}\right)+f_{3}(\mathrm{a}) \otimes y_{3}+\cdots+f_{h}(\mathrm{a}) \otimes y_{h} \in V$ (car a $\in V_{1}=E_{1}$ ), ce qui contredit la définition de $h$. Donc tous les endomorphismes $f_{i}$ sont nuls et, pour tout $x \in E_{1}, x \otimes y_{1} \in V$ et $h=1$. Alors, en fixant $x \in E_{1} \backslash\{0\}$, l'espace vectoriel $\left\{y \in E_{2} ; x \otimes y \in V\right\}$ est non nul et stable par le groupe $G_{2}$, donc égal à $E_{2}$. Donc $V=E_{1} \otimes E_{2}$.

Le lecteur intéressé pourra consulter [70], § 8.4.2 et [72], théorème 10 page 41, où se trouve d'autres preuves de ce lemme.

\section{Groupe unitaire}

Soient $\ell, N_{1}, \ldots, N_{\ell} \in \mathbb{N}^{*}$. Soit $\mathbf{U}$ le produit des groupes unitaires $U_{N_{i}+1}(\mathbb{C})$. Le groupe $\mathbf{U}$ agit sur l'espace des sections globales $E$ de

$$
\mathcal{O}_{\mathbb{P}_{\mathbb{C}}^{N_{1}} \times \cdots \times \mathbb{P}_{\mathbb{C}}^{N_{\ell}}}\left(D_{1}, \ldots, D_{\ell}\right)
$$

i.e. les polynômes multihomogènes de $\mathbb{P}_{\mathbb{C}}^{N_{1}} \times \cdots \times \mathbb{P}_{\mathbb{C}}^{N_{\ell}}$, à coefficients complexes, de multidegré $\left(D_{1}, \ldots, D_{\ell}\right)$. Par définition de la métrique de Fubini-Study, cette action est isométrique. Nous utiliserons (remarque 1, page 80) le :

Lemme II.3.10. L'action de $\mathbf{U}$ sur E est irréductible.

Démonstration. N'ayant pas de références précises pour cet énoncé, nous en proposons une démonstration «à la main ».

D'après le lemme précédent, il suffit de traiter le cas $\ell=1$. Nous allons montrer que $U_{N+1}(\mathbb{C})$ agit irréductiblement sur l'espace vectoriel $\mathbb{C}\left[X_{0}, \ldots, X_{N}\right]_{D}=\mathrm{H}^{0}\left(\mathbb{P}^{N}(\mathbb{C}), \mathcal{O}(D)\right)$. Nous raisonnons par récurrence sur $N$.

Pour $N=0$, c'est immédiat. Supposons le résultat vrai pour $N-1$.

Soient $V$ un sous-espace vectoriel non nul de $\mathbb{C}\left[X_{0}, \ldots, X_{N}\right]_{D}$ stable par $U_{N+1}(\mathbb{C})$ et $P \in V \backslash\{0\}$. L'action de la matrice diagonale $\operatorname{Diag}\left(e^{i \theta_{0}}, \ldots, e^{i \theta_{N}}\right)$ sur $P$ montre que $P\left(e^{i \theta_{0}} X_{0}, \ldots, e^{i \theta_{N}} X_{N}\right)$ appartient à $V$ pour tout $\left(\theta_{0}, \ldots, \theta_{N}\right) \in \mathbb{R}^{N+1}$. Cela signifie que tous les monômes apparaissant dans $P$ avec un coefficient non nul appartiennent à $V$. En particulier, il existe $\underline{h}:=\left(h_{0}, \ldots, h_{N}\right) \in \mathbb{N}^{N+1}$ de longueur $D$ tel que $X_{0}^{h_{0}} \cdots X_{N}^{h_{N}} \in V$. Parmi tous les monômes de ce type dans $V$, choisissons en un qui est de degré minimal par rapport à $X_{0}$. L'action de la matrice

$$
\left(\begin{array}{cc}
\left(\begin{array}{cc}
\cos \theta & -\sin \theta \\
\sin \theta & \cos \theta
\end{array}\right) & \left.\begin{array}{cccc} 
& & & \\
1 & 0 & \ldots & 0 \\
0 & 1 & \ldots & 0 \\
\vdots & \ddots & \ddots & \vdots \\
0 & \ldots & 0 & 1
\end{array}\right)
\end{array}\right)
$$

sur ce monôme implique :

$$
\forall \theta \in \mathbb{R}, \sum_{\substack{\alpha \in\left\{0, \ldots, h_{0}\right\} \\
\beta \in\left\{0, \ldots, h_{1}\right\}}}(-1)^{\alpha}\left(\begin{array}{c}
h_{0} \\
\alpha
\end{array}\right)\left(\begin{array}{c}
h_{1} \\
\beta
\end{array}\right)(\tan \theta)^{\beta-\alpha} X_{0}^{\alpha+\beta} X_{1}^{h_{0}+h_{1}-\alpha-\beta} X_{2}^{h_{2}} \cdots X_{N}^{h_{N}} \in V
$$

donc, en particulier, en prenant le coefficient de $(\tan \theta)^{h_{1}-1}$, on obtient

$$
\left(h_{1} X_{0}^{h_{0}+1} X_{1}^{h_{1}-1}-h_{0} X_{0}^{h_{0}-1} X_{1}^{h_{1}+1}\right) \cdot X_{2}^{h_{2}} \cdots X_{N}^{h_{N}} \in V .
$$

L'action de la matrice $\operatorname{Diag}\left(e^{i \theta}, 1,1, \ldots, 1\right)$ sur ce dernier polynôme implique que

$$
-h_{0} X_{0}^{h_{0}-1} X_{1}^{h_{1}+1} X_{2}^{h_{2}} \cdots X_{N}^{h_{N}} \in V
$$


donc, par minimalité, on a $h_{0}=0$. Ceci montre que $V \cap \mathbb{C}\left[X_{1}, \ldots, X_{N}\right]_{D}$ est non nul, et comme cet espace est stable par $U_{N}(\mathbb{C})$, on déduit de l'hypothèse de récurrence qu'il est égal à $\mathbb{C}\left[X_{1}, \ldots, X_{N}\right]_{D}$ tout entier. Soit $X_{1}^{\alpha_{1}} \cdots X_{N}^{\alpha_{N}} \in V$. L'action de la matrice (7) sur ce monôme et la formule du binôme impliquent que

$$
\sum_{\ell=0}^{\alpha_{1}}\left(\begin{array}{c}
\alpha_{1} \\
\ell
\end{array}\right) \cdot X_{0}^{\ell} X_{1}^{\alpha_{1}-\ell} X_{2}^{\alpha_{2}} \cdots X_{N}^{\alpha_{N}} \in V
$$

donc, pour tout $\ell \in\left\{0, \ldots, \alpha_{1}\right\}$, le polynôme $X_{0}^{\ell} X_{1}^{\alpha_{1}-\ell} X_{2}^{\alpha_{2}} \cdots X_{N}^{\alpha_{N}}$ appartient à $V$. Et par conséquent, en faisant varier $\underline{\alpha}$, l'espace $V$ est nécessairement égal à $\mathrm{H}^{0}\left(\mathbb{P}^{N}(\mathbb{C}), \mathcal{O}(D)\right)$.

\section{Un calcul de hauteur}

Dans ce paragraphe, nous calculons la hauteur d'un élément algébrique de $\mathbb{P}^{N}(\overline{\mathbb{Q}})$ par rapport au fibré $\mathcal{O}_{\mathbb{P}^{N}}(1)$, muni des métriques de Fubini-Study aux places infinies. Rappelons que, par définition, si $\mathbf{x} \in \mathbb{P}^{N}(\overline{\mathbb{Q}})=\mathbb{P}^{N}(\overline{\mathbb{Z}})$, la hauteur de $\mathbf{x}$, notée $h_{\overline{\mathcal{O}(1)}}(\mathbf{x})$, est le degré d'Arakelov normalisé de $\mathbf{x}^{*} \mathcal{O}_{\mathbb{P}^{N}}(1)$ :

$$
h \overline{\mathcal{O}(1)}(\mathbf{x}):=\widehat{\operatorname{deg}}_{\mathrm{n}} \overline{\mathbf{x}^{*} \mathcal{O}(1)} .
$$

Lemme II.3.11 ( [76]). Si $\mathbf{x}=\left(x_{0}: \cdots: x_{N}\right) \in \mathbb{P}^{N}(\overline{\mathbb{Q}})$ alors

$$
h_{\overline{\mathcal{O}(1)}}(\mathbf{x})=\frac{1}{[\mathbf{L}: \mathbb{Q}]} \log \frac{\prod_{\sigma: \mathbf{L} \hookrightarrow \mathbb{C}}\left(\sum_{i=0}^{N}\left|\sigma\left(x_{i}\right)\right|^{2}\right)^{1 / 2}}{\mathrm{~N}_{\mathbf{L} \mid \mathbb{Q}}\left(x_{0} \cdot \mathcal{O}_{\mathbf{L}}+\cdots+x_{N} \cdot \mathcal{O}_{\mathbf{L}}\right)}
$$

où $\mathbf{L}$ est un corps de nombres contenant $x_{0}, \ldots, x_{N}$ et où, si I désigne un idéal (fractionnaire) de $\mathcal{O}_{\mathbf{L}}$, le nombre rationnel $\mathrm{N}_{\mathbf{L} \mid \mathbb{Q}}(I)$ désigne la norme de $I$.

Démonstration. Nous pouvons supposer que les coordonnées de $\mathrm{x}$ appartiennent à $\mathcal{O}_{\mathbf{L}}$. Posons

$$
\mathfrak{n}:=\Gamma\left(\operatorname{Spec} \mathcal{O}_{\mathbf{L}}, \mathbf{x}^{*} \mathcal{O}(1)\right)=x_{0} \cdot \mathcal{O}_{\mathbf{L}}+\cdots+x_{N} \cdot \mathcal{O}_{\mathbf{L}} .
$$

Par définition (voir annexe B, définition B.1.2), on a

$$
h_{\overline{\mathcal{O}(1)}}(\mathbf{x})=\frac{1}{[\mathbf{L}: \mathbb{Q}]}\left(\log \operatorname{Card}\left(\mathfrak{n} / s \cdot \mathcal{O}_{\mathbf{L}}\right)-\sum_{\sigma: \mathbf{L} \rightarrow \mathbb{C}} \log \|s\|_{\overline{\mathcal{O}(1)}, \sigma}\right)
$$

où $s \in \mathfrak{n} \backslash\{0\}$. Parmi les coordonnées $x_{0}, \ldots, x_{N}$, une au moins est non nulle, par exemple $x_{0}$. Alors, de la suite exacte d'anneaux finis

$$
0 \longrightarrow \mathfrak{n} / x_{0} \mathcal{O}_{\mathbf{L}} \longrightarrow \mathcal{O}_{\mathbf{L}} / x_{0} \mathcal{O}_{\mathbf{L}} \longrightarrow \mathcal{O}_{\mathbf{L}} / \mathfrak{n} \longrightarrow 0
$$

on déduit

$$
\operatorname{card}\left(\mathcal{O}_{\mathbf{L}} / x_{0} \mathcal{O}_{\mathbf{L}}\right)=\operatorname{card}\left(\mathcal{O}_{\mathbf{L}} / \mathfrak{n}\right) \times \operatorname{card}\left(\mathfrak{n} / x_{0} \mathcal{O}_{\mathbf{L}}\right)
$$

donc

$$
\operatorname{card}\left(\mathfrak{n} / x_{0} \cdot \mathcal{O}_{\mathbf{L}}\right)=\frac{\prod_{\sigma: \mathbf{L} \hookrightarrow \mathbb{C}}\left|\sigma\left(x_{0}\right)\right|}{\mathrm{N}_{\mathbf{L} \mid \mathbb{Q}}\left(x_{0} \cdot \mathcal{O}_{\mathbf{L}}+\cdots+x_{N} \cdot \mathcal{O}_{\mathbf{L}}\right)} .
$$

Par ailleurs, par définition de la métrique de Fubini-Study (§ II.3.b. ), on a :

$$
\left\|x_{0}\right\|_{\overline{\mathcal{O}(1)}, \sigma}=\frac{\left|\sigma\left(x_{0}\right)\right|}{\left(\sum_{j=0}^{N}\left|\sigma\left(x_{j}\right)\right|^{2}\right)^{1 / 2}}
$$


et donc

$$
\sum_{\sigma: \mathbf{L} \hookrightarrow \mathbb{C}} \log \left\|x_{0}\right\|_{\overline{\mathcal{O}(1)}, \sigma}=\log \frac{\prod_{\sigma: \mathbf{L} \hookrightarrow \mathbb{C}}\left|\sigma\left(x_{0}\right)\right|}{\prod_{\sigma: \mathbf{L} \hookrightarrow \mathbb{C}}\left(\sum_{i=0}^{N}\left|\sigma\left(x_{i}\right)\right|^{2}\right)^{1 / 2}} .
$$

Les égalités (9), (10) et (11) permettent de conclure.

Remarque : ce lemme nous permet donc de voir que la hauteur par rapport au fibré hermitien $\overline{\mathcal{O}(1)}$ est comparable à la hauteur de Weil (logarithmique absolue), à une fonction bornée près :

$$
h_{\text {Weil }}(\mathbf{x}) \leq h_{\overline{\mathcal{O}(1)}}(\mathbf{x}) \leq \frac{1}{2} \log (N+1)+h_{\text {Weil }}(\mathbf{x}) .
$$

Nous utiliserons cette majoration de $h_{\overline{\mathcal{O}(1)}}(\mathbf{x})$ dans le lemme II.5.5, afin d'exprimer la minoration de $|\Lambda|$ en fonction de la hauteur de Weil de $u_{0}$. Ce n'est qu'une question de présentation.

\section{$\S$ 3.d. Méthode des pentes}

Cette section reprend, pour la commodité du lecteur, l' inégalité de pentes (qui est au cœur de la méthode du même nom) de J.-B. Bost, exposée dans [19] et [22]. La définition des notations (《standard ») employées dans ce paragraphe se trouve à l'annexe B.

Soient $K$ un corps de nombres, $\mathscr{E}$ et $F$ deux $\mathcal{O}_{K}$-modules de type fini et projectifs. Soit $\varphi: \mathscr{E} \rightarrow F$ un morphisme de $\mathcal{O}_{K}$-modules. On suppose que $\mathscr{E}$ est muni de métriques à l'infini et on note $\overline{\mathscr{E}}$ le fibré ainsi métrisé. Considérons une filtration de $F$

$$
\{0\}=F_{\mathcal{N}} \subseteq F_{\mathcal{N}-1} \subseteq \cdots \subseteq F_{0}=F
$$

par des sous-modules dont les quotients

$$
G_{i}=F_{i} / F_{i+1}
$$

sont sans torsion, donc des $\mathcal{O}_{K^{-}}$modules projectifs de type fini et métrisés $\overline{\widetilde{G}}_{i}$. Le module $G_{i}$ est alors muni des métriques induites par celles de $\widetilde{G}_{i}$. On notera $\mathscr{E}_{i}=\varphi^{-1}\left(F_{i}\right)$ (que l'on munit bien sûr des métriques induites par celles de $\mathscr{E}$ ) et $\widetilde{\varphi}_{i}: \mathscr{E}_{i} \rightarrow \widetilde{G}_{i}$ le morphisme composé de $\varphi$, de la projection canonique de $F_{i}$ sur $G_{i}$ et de l'inclusion $G_{i} \subseteq \widetilde{G}_{i}$. Si $\varphi$ est injective, alors :

$$
\widehat{\operatorname{deg}}_{\mathrm{n}}(\overline{\mathscr{E}}) \leq \sum_{i=0}^{\mathcal{N}-1}\left(\operatorname{rg}\left(\mathscr{E}_{i}\right)-\operatorname{rg}\left(\mathscr{E}_{i+1}\right)\right)\left(\widehat{\mu}_{\max }\left(\overline{\widetilde{G}_{i}}\right)+h\left(\widetilde{\varphi}_{i}\right)\right)
$$

où $\|\cdot\|_{\sigma}$ est la norme d'opérateur et $h\left(\widetilde{\varphi}_{i}\right)=\frac{1}{[K: \mathbb{Q}]} \sum_{\sigma: K \rightarrow \mathbb{C}} \log \left\|\widetilde{\varphi}_{i}\right\|_{\sigma}$.

Preuve succincte. Comme $\varphi$ est injective, le $\mathcal{O}_{K}$-module $\mathscr{E}_{i} / \mathscr{E}_{i+1}$ s'injecte dans $\widetilde{G}_{i}$ donc (proposition 4.3 de [19])

$$
\widehat{\mu}_{\max }\left(\overline{\mathscr{E}_{i} / \mathscr{E}_{i+1}}\right) \leq \widehat{\mu}_{\max }\left(\overline{\widetilde{G}_{i}}\right)+h\left(\widetilde{\varphi}_{i}\right)
$$

et par conséquent

$$
\widehat{\operatorname{deg}}_{\mathrm{n}}\left(\overline{\mathscr{E}_{i} / \mathscr{E}_{i+1}}\right) \leq \operatorname{rg}\left(\mathscr{E}_{i} / \mathscr{E}_{i+1}\right)\left(\widehat{\mu}_{\max }\left(\overline{\widetilde{G}_{i}}\right)+h\left(\widetilde{\varphi}_{i}\right)\right)
$$

puis on somme sur $i$. 
Dans le cas particulier simple où $\mathscr{E}, F, G_{i}$ sont des $\mathcal{O}_{K^{-}}$modules libres de type fini et munis de métriques hermitiennes «standard ( après choix de $\mathcal{O}_{K}$-bases), l'inégalité (12) est l'analogue (en dimension 1) de la formule du produit (comme peut l'être le lemme de Liouville, en transcendance). Cette formule et le formalisme qui l'accompagne établissent un lien entre l'Approximation Diophantienne et la Géométrie d'Arakelov.

\section{$\S 4$. Schéma tactique de la preuve}

Tout d'abord, nous utiliserons une version affaiblie de l'inégalité (12) :

$$
\widehat{\operatorname{deg}}_{\mathrm{n}} \overline{\mathscr{E}} \leq\left(\operatorname{rg} \mathscr{E}-\operatorname{rg} \mathscr{E}_{i_{0}}\right) \max _{0 \leq i \leq i_{0}-1}\left\{\widehat{\mu}_{\max }\left(\widetilde{\widetilde{G}}_{i}\right)+h\left(\widetilde{\varphi}_{i}\right)\right\}+\left(\operatorname{rg} \mathscr{E}_{i_{0}}\right) \max _{i_{0} \leq i \leq \mathcal{N}}\left\{\widehat{\mu}_{\max }\left(\widetilde{\widetilde{G}}_{i}\right)+h\left(\widetilde{\varphi}_{i}\right)\right\}
$$

où l'entier $i_{0}$ sera choisi assez grand pour que la quantité

$$
\max _{i_{0} \leq i \leq \mathcal{N}}\left\{\widehat{\mu}_{\max }\left(\widetilde{\widetilde{G}}_{i}\right)+h\left(\widetilde{\varphi}_{i}\right)\right\}
$$

soit négative, et, cependant, pas trop grand pour que le rang de $\mathscr{E}_{i_{0}}$ soit de l'ordre de celui de $\mathscr{E}$ (en l'occurrence $\operatorname{rg} \mathscr{E}_{i_{0}} \geq \frac{\operatorname{rg} \mathscr{E}}{2}$, voir lemme II.5.6, page 85). Le problème de l'injectivité de $\varphi$ sera résolue avec le lemme de zéros [63] (§ II.5.a.). Par ailleurs, le choix de $\overline{\mathscr{E}}$ permet de calculer (à l'aide du théorème II.3.2) son degré d'Arakelov normalisé. Mais la seule information que nous garderons de ce calcul est l'inégalité

$$
\widehat{\operatorname{deg}}_{\mathrm{n}} \overline{\mathscr{E}} \geq-\frac{h_{F}(\mathbf{A})}{2} \operatorname{rg} \mathscr{E}
$$

vérifiée dès qu'un certain entier $D_{1}$ associé à $\mathscr{E}$ est supérieur ou égal à 3 . Par conséquent, c'est l'inégalité suivante (conséquence de (13)) qui sera utilisée :

$$
-\frac{h_{F}(\mathbf{A})}{2} \leq \max _{0 \leq i \leq i_{0}-1}\left\{\widehat{\mu}_{\max }\left(\widetilde{\widetilde{G}}_{i}\right)+h\left(\widetilde{\varphi}_{i}\right)\right\}+\frac{1}{2} \max _{i_{0} \leq i \leq \mathcal{N}}\left\{\widehat{\mu}_{\max }\left(\widetilde{\widetilde{G}}_{i}\right)+h\left(\widetilde{\varphi}_{i}\right)\right\} .
$$

Comme dans la première partie, nous raisonnerons par l'absurde en supposant $|\Lambda|$ très petit, ce qui nous permettra d'obtenir un majorant, également très petit, de la norme (relative au plongement $\sigma_{0}$ de $K$ dans $\mathbb{C}$ ) de $\widetilde{\varphi}_{i}$, pour $i \geq i_{0}$. La hauteur $h\left(\widetilde{\varphi}_{i}\right)$ sera alors « fortement 》 négative, ce qui conduira à une contradiction avec l'inégalité (15). On pourra noter le parfait mimétisme (une place $\sigma_{0}$ privilégiée) avec l'utilisation usuelle de la formule du produit en Transcendance (inégalité de Liouville).

Les définitions précises des objets $\mathscr{E}, F, \varphi, G_{i} \ldots$ qui interviennent dans l'inégalité de pentes sont données aux paragraphes II.5.a. et II.5.a. . Grosso modo, le morphisme $\varphi$ associe à une section $s$ (d'un certain fibré en droites) un nombre fini de ses jets * le long de l'hyperplan $W$ en les multiples du point $\mathbf{p}$. Il y a dans ce texte deux difficultés techniques particulières (en comparaison avec [19], par exemple) qui tiennent au souci que nous avons de « ménager »

${ }^{*}$ Soient $\mathfrak{M}$ un espace vectoriel complexe de dimension $d$ et $\mathfrak{m}=\left(\mathfrak{m}_{1}, \ldots, \mathfrak{m}_{d}\right)$ une base de $\mathfrak{M}$. Soit $f: \mathfrak{M} \rightarrow \mathbb{C}$ une fonction analytique (au voisinage de 0 ). Le jet d'ordre $T$ de $f$ en 0 est l'application de $\mathfrak{M}$ dans $\mathbb{C}$ qui à $t_{1} \mathfrak{m}_{1}+\cdots+t_{d} \mathfrak{m}_{d}$ associe $\sum_{|\underline{i}|=T} \frac{1}{\underline{i} !}\left(\frac{\partial}{\partial z_{1}}\right)^{i_{1}} \cdots\left(\frac{\partial}{\partial z_{d}}\right)^{i_{d}} f\left(z_{1} \mathfrak{m}_{1}+\cdots+z_{d} \mathfrak{m}_{d}\right)(0) \underline{\underline{i}}$. Contrairement aux coefficients de Taylor, cette application jet est indépendante du choix de la base $\mathfrak{m}$. 
l'hyperplan, et, en particulier, sa hauteur, afin d'obtenir, comme dans la première partie, une minoration de $|\Lambda|$ optimale en $h(W)$. C'est pourquoi, d'une part, nous devons être soigneux pour les estimations des normes ultramétriques des $\widetilde{\varphi}_{i}$, en montrant qu'il est possible de trouver un «bon » dénominateur pour les jets, de manière à ce que les $\mathcal{O}_{K}$-modules $\widetilde{\varphi}_{i}\left(\mathscr{E}_{i}\right)$ soient (à quelques détails près) des sous-modules de $\mathcal{O}_{K}^{(\mathbb{N})}$. C'est pourquoi, d'autre part, nous composerons $\varphi$ avec la projection $t_{\mathbf{G}} \rightarrow W$ afin de ne pas faire apparaître la pente maximale de $\overline{S^{T} W^{v}}$, ce qui introduit un terme $T h(W) \gtrsim h(W)^{2}$ funeste à notre dessein, dans l'évaluation des pentes maximales $\widehat{\mu}_{\text {max }}\left(\widetilde{G}_{i}\right)$. Cet artifice déplace le problème lors de l'évaluation des hauteurs $h\left(\widetilde{\varphi}_{i}\right)$, où, alors, par un calcul adéquat et grâce à l'astuce de N. HiRATA-KoHNO, nous montrerons que la quantité $T h(W)$ peut être remplacée par min $\left(D_{0}, T\right) h(W)$ (et, bien sûr, $D_{0}$ sera un paramètre $\left.\leq T\right)$.

\section{§ 5. Démonstration du théorème}

Remarques liminaires :

1. Dans la mesure où le nombre de paramètres est relativement restreint, il est (souvent) plus limpide pour la démonstration d'une proposition intermédiaire de conserver les paramètres $D_{0}, D_{1}, T$ etc... indéfinis, pour ne les remplacer par leur valeur qu'au dernier moment. Cela facilite à la fois la vérification des énoncés et la possibilité pour le lecteur de modifier notre choix de paramètres avec un effort moindre. Par ailleurs, le lecteur qui suivrait les (nombreux) calculs de cette démonstration constaterait que les inégalités sont très larges pour les constantes numériques. Ainsi, outre la simplification des expressions, nous espérons nous prémunir ainsi d'une erreur de calcul (locale) sans remettre en cause la validité du théorème II.2.1.

2. Pour la démonstration, nous pouvons supposer que les nombres $\beta_{i}$ sont des entiers algébriques. En effet, on se ramène aisément à ce cas en multipliant la forme linéaire $\Lambda$ par un dénominateur $\left(\in \mathbb{N}^{*}\right)$ des $\beta_{i}(1 \leq i \leq d)$, dénominateur qui peut être choisi plus petit que $b$ (défini au théorème II.2.1).

3. Dans tout ce qui suit, nous supposerons que $(\mathbf{A}, L)$ est principalement polarisée (voir remarque qui suit le théorème II.2.1).

\section{§ 5.a. Choix des paramètres, du fibré hermitien de départ et de la filtration}

\section{Liste des paramètres}

Dans ce paragraphe, le polynôme de Hilbert-Samuel de $\mathbf{G}$ sera relatif aux plongements de $\mathbb{G}_{\mathrm{a}}$ dans $\mathbb{P}^{1}$ usuel et à celui de $\mathbf{A}$ dans un espace projectif $\mathbb{P}^{N}$, déterminé par le fibré très ample $L^{\otimes 3}$. Ainsi, via le plongement induit de $\mathbf{G}$ dans $\mathbb{P}^{1} \times \mathbb{P}^{N}$, le degré de $\mathbf{G}$ vaut $3^{d} \operatorname{deg}_{L} \mathbf{A}=3^{d} d$ !

Soient $S_{0}<S$ des entiers naturels, $\widetilde{D}_{0}, \widetilde{D}_{1}, \widetilde{T}, x, x_{0}, C_{0}$ des réels strictement positifs. Comme dans la première partie, $\widetilde{D}_{0}$ et $\widetilde{D}_{1}$ sont des paramètres fixant des degrés (partiels) de polynômes, $\widetilde{T}$ et $\widetilde{T}_{0}$ sont des « ordres de dérivations », $S$ et $S_{0}$ sont des « nombres de points $\gg$ dans l'extrapolation, $x$ et $x_{0}$ sont des variables d'ajustement vis-à-vis du lemme de 
zéros. Posons $T^{\#}:=x_{0} \widetilde{T}$ et $T:=\left[T^{\#}\right]$. Nous supposerons que $S+1 \geq(3 / 2)^{d} C_{0}$. Choisissons un réel

$$
x_{0} \geq \frac{(S+1)}{C_{0}(\operatorname{deg} \mathbf{G}) \widetilde{D}_{0}} \times\left(\frac{\widetilde{T}}{\widetilde{D}_{1}}\right)^{d} .
$$

Lorsque $\mathbf{G}^{\prime}$ est un sous-groupe de $\mathbf{G}$, nous notons $r^{\prime}$ la codimension de $\mathbf{G}^{\prime}$ dans $\mathbf{G}$. Posons alors

$$
x:=\inf _{\substack{\mathbf{G}^{\prime} \subseteq \mathbf{G},{ }_{\mathbf{G}^{\prime}} \subseteq W \\ \mathbf{G}^{\prime} \text { connexe }}}\left\{\left(\frac{\widetilde{T}}{\widetilde{D}_{1}}\right)^{r^{\prime}-1} \times \frac{(S+1) \operatorname{deg}_{L} \mathbf{G}^{\prime}}{3^{r^{\prime}-1} C_{0} \widetilde{D}_{0} \operatorname{deg}_{L} \mathbf{A}}\right\} .
$$

Cette borne inférieure est un minimum (c'est immédiat car $r^{\prime}$ et $\operatorname{deg}_{L} \mathbf{G}^{\prime}$ sont des entiers naturels). Notons $\widetilde{\mathbf{G}}$ un groupe (algébrique, connexe) pour lequel $x$ est atteint. L'hypothèse sur $x_{0}$ implique $x \leq x_{0}$, en prenant $\mathbf{G}^{\prime}=\{0\}$. Posons $D_{1}:=\left[x_{0} \widetilde{D}_{1}\right], D_{0}:=\left[x \widetilde{D}_{0}\right]$ et

$$
D_{0}^{\#}=x \widetilde{D}_{0}=\left(\frac{\widetilde{T}}{\widetilde{D}_{1}}\right)^{\widetilde{r}-1} \times \frac{(S+1) \operatorname{deg}_{L} \widetilde{\mathbf{G}}}{3^{\widetilde{r}-1} C_{0} \operatorname{deg}_{L} \mathbf{A}} .
$$

On notera que $\widetilde{r}$, la codimension de $\widetilde{\mathbf{G}}$ dans $\mathbf{G}$, est strictement supérieure à 1 car sinon $\operatorname{dim} \widetilde{\mathbf{G}}=\operatorname{dim} \mathbf{G}-1=\operatorname{dim} W$ et donc $W=t_{\widetilde{\mathbf{G}}}$, ce qui est exclu à cause de la transversalité de $W$ dans $t_{\mathbb{G}_{\mathrm{a}}} \oplus t_{\mathbf{A}_{K}}$. Comme $S+1 \geq(3 / 2)^{d} C_{0}$ par hypothèse, l'entier $D_{0}$ est non nul.

Proposition II.5.1. Si

1. $D_{1}$ est un entier non nul (i.e. $x_{0} \widetilde{D}_{1} \geq 1$ )

2. $C_{0}>4^{d} d$ !

3. $\widetilde{T} \geq 6^{d+1} d !\left(\operatorname{deg}_{L} \mathbf{A}\right) \max \left\{\widetilde{D}_{1}, \frac{\widetilde{D}_{0}}{S+1}\right\}$

alors il n'existe aucune section non-nulle de $\mathrm{H}^{0}\left(\mathbb{P}^{1} \times \mathbf{A}, \mathcal{O}\left(D_{0}\right) \otimes L^{\otimes 3 D_{1}}\right)$ qui s'annule le long de $W$ à l'ordre $(d+1) T$ en les points de $\Gamma_{\mathbf{p}}((d+1) S)$.

Démonstration. Si nous notons $i: \mathbf{A} \hookrightarrow \mathbb{P}^{N}$, le plongement induit par le fibré très ample $L^{\otimes 3}$, la section $s \in \Gamma\left(\mathbb{P}^{1}, \mathcal{O}\left(D_{0}\right)\right) \otimes \Gamma\left(\mathbf{A}, i^{*} \mathcal{O}_{\mathbb{P}^{N}}\left(D_{1}\right)\right)$ provient* d'un polynôme $P$, bihomogène de bidegré $\left(D_{0}, D_{1}\right)$. Si la proposition II.5.1 est fausse, ce polynôme s'annule à l'ordre $(d+1) T$ le long de $W$ en $\Gamma_{\mathbf{p}}((d+1) S)$. D'après le lemme de zéros dans les groupes algébriques commutatifs de Philippon [63], il existe un sous-groupe connexe et propre $\mathbf{G}^{\star}$ de $\mathbf{G}$ tel que

$$
T^{\operatorname{codim}_{W} W \cap t_{\mathbf{G}^{\star}}} \times \operatorname{card}\left(\frac{\Gamma_{\mathbf{p}}(S)+\mathbf{G}^{\star}(K)}{\mathbf{G}^{\star}(K)}\right) \times \mathscr{H}\left(\mathbf{G}^{\star} ; D_{0}, D_{1}\right) \leq 2^{d} d ! \mathscr{H}\left(\mathbf{G} ; D_{0}, D_{1}\right) .
$$

Nous allons distinguer plusieurs cas à l'aide du fait suivant :

Fait : Si $\mathbf{G}^{\star}$ est un sous-groupe algébrique connexe de $\mathbb{G}_{\mathrm{a}} \times \mathbf{A}$ alors il existe une sous-variété abélienne $\mathbf{A}^{\star}$ de $\mathbf{A}$ telle que

${ }^{*}$ Comme $L^{\otimes 3}$ est normalement engendré, le sous-schéma fermé $i(\mathbf{A})$ de $\mathbb{P}^{N}$ est projectivement normal, ce qui signifie que, pour tout entier $n \geq 1$, le morphisme de restriction naturel

$$
\mathrm{H}^{0}\left(\mathbb{P}^{N}, \mathcal{O}_{\mathbb{P}^{N}}(n)\right) \rightarrow \mathrm{H}^{0}\left(\mathbf{A}, L^{\otimes 3 n}\right)
$$

est surjectif (voir [12], pp. $190-193$ ). 


\section{Le cas abélien non-homogène}

(1) $\mathbf{G}^{\star}=\{0\} \times \mathbf{A}^{\star}$

$\mathrm{Ou}$

(2) $\mathbf{G}^{\star}=\mathbb{G}_{\mathrm{a}} \times \mathbf{A}^{\star}$.

- Si $t_{\mathbf{G}^{\star}} \subseteq W$, on a $\operatorname{codim}_{W} W \cap t_{\mathbf{G}^{\star}}=\operatorname{codim}_{\mathbf{G}} \mathbf{G}^{\star}-1=r^{\star}-1$ et, nécessairement nous sommes dans le cas (1). Alors l'inégalité (16) devient

$$
\left(\frac{T}{D_{1}}\right)^{r^{\star}-1} \cdot \frac{(S+1) \operatorname{deg}_{L} \mathbf{G}^{\star}}{2^{d} d ! \times 3^{r^{\star}-1} \operatorname{deg}_{L} \mathbf{A}} \leq D_{0}
$$

Comme $T \geq 1$, on a $T \geq \frac{1}{2} x_{0} \widetilde{T}$ donc $T / D_{1} \geq \widetilde{T} /\left(2 \widetilde{D}_{1}\right)$ et (17) devient

$$
x \geq \frac{1}{4^{d} d !}\left(\frac{\widetilde{T}}{\widetilde{D}_{1}}\right)^{r^{\star}-1} \cdot \frac{(S+1) \operatorname{deg}_{L} \mathbf{G}^{\star}}{3^{r^{\star}-1} \widetilde{D}_{0} \operatorname{deg}_{L} \mathbf{A}}
$$

ce qui contredit la définition de $x$ car $C_{0}>4^{d} d$ !.

- Si $t_{\mathbf{G}^{\star}} \nsubseteq W$, les cas (1) et (2) sont possibles :

Cas (1) : On a

$$
T^{r^{\star}}(S+1) \leq 2^{d} d ! 3^{r^{\star}-1} \cdot \frac{\operatorname{deg}_{L} \mathbf{A}}{\operatorname{deg}_{L} \mathbf{G}^{\star}} \cdot D_{0} D_{1}^{r^{\star}-1}
$$

ce qui implique

$$
x_{0}(S+1)\left(\frac{\widetilde{T}}{2 \widetilde{D}_{1}}\right)^{r^{\star}} \leq 2^{d} d ! 3^{r^{\star}-1} \cdot x \cdot \frac{\left(\operatorname{deg}_{L} \mathbf{A}\right) \widetilde{D}_{0}}{\left(\operatorname{deg}_{L} \mathbf{G}^{\star}\right) \widetilde{D}_{1}}
$$

donc, comme $r^{\star} \geq 1$, on a

$$
x \geq \frac{\widetilde{T}(S+1) x_{0}}{2^{d+1} d ! \times 3^{r^{\star}-1} \widetilde{D}_{0} \operatorname{deg}_{L} \mathbf{A}}>x_{0} .
$$

Cette inégalité contredit la définition de $x$.

Cas (2) : D'après l'inégalité (16), on a

$$
\left(\frac{T}{D_{1}}\right)^{r^{\star}} \operatorname{deg}_{L} \mathbf{A}^{\star} \leq 6^{d} d ! \operatorname{deg}_{L} \mathbf{A}
$$

et comme $T / D_{1} \geq \widetilde{T} /\left(2 \widetilde{D}_{1}\right)$ et $r^{\star} \geq 1$, on a

$$
\frac{\widetilde{T}}{\widetilde{D}_{1}} \leq 2 \times 6^{d} d ! \operatorname{deg}_{L} \mathbf{A}
$$

ce qui est impossible.

Dans aucun cas, le groupe $\mathbf{G}^{\star}$ ne peut exister et la proposition est démontrée. 


\section{Choix du fibré hermitien}

Par choix du corps $k$, il existe un modèle de Moret-Bailly

$$
\left(\pi: \mathscr{A} \rightarrow \operatorname{Spec} \mathcal{O}_{k}, \overline{\mathscr{L}},\left\{\varepsilon_{m \mathbf{p}}: \operatorname{Spec} \mathcal{O}_{k} \rightarrow \mathscr{A}\right\}_{m=0, \ldots,(d+1) S}\right)
$$

de $\left(\mathbf{A}, L, \Gamma_{\mathbf{p}}((d+1) S)\right)$. Le schéma $\mathscr{X}:=\mathbb{P}_{\mathcal{O}_{k}}^{1} \times \mathscr{A}$ est lisse et quasi-projectif* sur Spec $\mathcal{O}_{k}$, et le schéma en groupe $\mathscr{G}:=\mathbb{G}_{\mathrm{a}} \times \mathscr{A}$ est un ouvert (lisse sur Spec $\mathcal{O}_{k}$ ) de $\mathscr{X}$. Sur ce schéma, nous considérons le fibré en droites

$$
\mathscr{M}:=\mathcal{O}_{\mathbb{P}_{\mathcal{O}_{k}}^{1}}\left(D_{0}\right) \otimes \mathscr{L}^{\otimes 3 D_{1}}
$$

que nous munissons, aux places infinies, des métriques hermitiennes induites par produit tensoriel et pull-back des métriques de Fubini-Study sur $\mathcal{O}(1)$ et des métriques du cube sur $\mathscr{L}$. Notons $\mathscr{E}:=\Gamma(\mathscr{X}, \mathscr{M})$ le $\mathcal{O}_{k}$-module projectif de type fini des sections globales de $\mathscr{M}$. Il est de rang $\left(D_{0}+1\right)\left(3 D_{1}\right)^{d}$ (ici $\mathrm{h}^{0}(\mathbf{A}, L)$, la dimension de l'espace des sections globales de $L$ - comme $L$ est ample, c'est aussi égal à la caractéristique d'Euler-Poincaré $\chi(\mathbf{A}, L)$ de $(\mathbf{A}, L)$ - vaut 1 car la polarisation est principale). Nous munissons $\mathscr{E}$ d'une structure de fibré hermitien $\overline{\mathscr{E}}:=\left(\mathscr{E},\left(\|\cdot\|_{\overline{\mathscr{E}}, \sigma}\right)_{\sigma: k \rightarrow \mathbb{C}}\right)$ en définissant, pour tout plongement $\sigma: k \rightarrow \mathbb{C}$, et tout élément $s \in \mathscr{E}$, la norme hermitienne :

$$
\|s\|_{\mathscr{E}, \sigma}^{2}:=\int_{\mathscr{X}_{\sigma}(\mathbb{C})}\|s(x)\|_{\mathscr{M}_{, \sigma}}^{2} \mathrm{~d} \mu_{\sigma}(x)
$$

où $\mathrm{d} \mu_{\sigma}$ est la mesure de probabilité sur $\mathscr{X}_{\sigma}(\mathbb{C})$, invariante par le produit du groupe unitaire $\mathbf{U}_{2}(\mathbb{C})$ et $d u$ groupe des translations de $\mathbf{A}_{\sigma}(\mathbb{C})\left(\mathrm{d} \mu_{\sigma} \propto \omega_{\mathbb{P}^{1}} \wedge c_{1}\left(L_{\sigma}\right)^{d}\right)$. Nous aurons besoin également de la norme infinie :

$$
\|s\|_{\infty, \sigma}:=\sup _{x \in \mathscr{X}_{\sigma}(\mathbb{C})}\|s(x)\|_{\mathscr{\mathscr { M }}, \sigma} .
$$

En comparant ces normes et grâce au travail [39] de P. GRAfTIEAUx, nous avons l'inégalité suivante, dite « de Gromov » (voir note *, p. 68) :

Lemme II.5.2. On a

$$
\frac{1}{[K: \mathbb{Q}]} \sum_{\sigma: K \rightarrow \mathbb{C}} \log \sup _{s_{\sigma} \in \mathscr{E}_{\sigma} \backslash\{0\}}\left\{\frac{\left\|s_{\sigma}\right\|_{\infty, \sigma}}{\left\|s_{\sigma}\right\|_{\overline{\mathscr{E}}_{,}}}\right\} \leq \log \left(D_{0}+1\right)+d \log \left(D_{1}\right)+\frac{d^{2}}{4} \max \left\{1, h_{F}(\mathbf{A})\right\}+4 d^{4}
$$

Démonstration. La démonstration de ce lemme ne pose aucune difficulté si on utilise la proposition 2.11 de [39] (c'est ici que l'hypothèse « principalement polarisée » intervient). Plus précisément, avec les notations de cette proposition, le lemme s'obtient en décomposant $s_{\sigma}$ dans la base orthonormée

$$
\left(\sqrt{\frac{\left(D_{0}+1\right) !}{\left(D_{0}-h\right) ! h !}} X_{0}^{D_{0}-h} X_{1}^{h} \otimes s_{\ell, \sigma}\right)_{\substack{h=0 \ldots D_{0} \\ \ell=1 \ldots\left(3 D_{1}\right)^{d}}}
$$

de $\mathscr{E}_{\sigma}$.

*Il n'est pas nécessaire ici d'avoir un schéma projectif car le degré d'Arakelov des sections d'une puissance de $\mathcal{L}$ est calculable explicitement (voir proposition II.5.3 ci-dessous). Dans [22], J.-B. Bost présente une minoration du degré (d'Arakelov) de ce type de fibré lorsque $\mathscr{A}$ est remplacé par un schéma intègre, de type fini, plat et propre sur Spec $\mathbb{Z}$. De la sorte, si nous ne cherchions pas des constantes explicites, la (simple) adhérence schématique de $\mathbf{A}$ dans un espace projectif sur $\mathcal{O}_{k}$ aurait pu se substituer au MB-modèle... 
La proposition suivante est le calcul explicite du degré d'Arakelov normalisé de $\overline{\mathscr{E}}$ (voir [19], $\S$ A.2, page 147, pour la définition de ce réel).

Proposition II.5.3. Le degré d'Arakelov normalisé de $\overline{\mathscr{E}}$ vaut

$$
\widehat{\operatorname{deg}}_{\mathrm{n}} \overline{\mathscr{E}}=\frac{1}{2} \log \left\{\prod_{h=0}^{D_{0}} \frac{\left(D_{0}+1\right) !}{h !\left(D_{0}-h\right) !}\right\}+\left(3 D_{1}\right)^{d}\left(-\frac{1}{2} h_{F}(\mathbf{A})+\frac{1}{4} \log \frac{\left(3 D_{1}\right)^{d}}{(2 \pi)^{d}}\right) .
$$

Démonstration. Par définition des métriques sur $\mathscr{E}$, on a

$$
\widehat{\operatorname{deg}}_{\mathrm{n}} \overline{\mathscr{E}}=\widehat{\operatorname{deg}}_{\mathrm{n}} \overline{\mathrm{H}^{0}\left(\mathbb{P}^{1}, \mathcal{O}\left(D_{0}\right)\right)}+\widehat{\operatorname{deg}}_{\mathrm{n}} \overline{\mathrm{H}^{0}\left(\mathscr{A}, \mathscr{L}^{\otimes 3 D_{1}}\right)} .
$$

Le calcul du second terme de la somme est une conséquence immédiate du iii) du théorème II.3.2. Quant au premier terme, considérons

$$
s=\bigwedge_{h=0}^{D_{0}}\left(X_{0}^{h} X_{1}^{D_{0}-h}\right)
$$

une $\mathcal{O}_{k}$-base du module $\operatorname{det} \mathrm{H}^{0}\left(\mathbb{P}_{\mathcal{O}_{k}}^{1}, \mathcal{O}\left(D_{0}\right)\right)$. Par définition du degré d'Arakelov, on a

$$
\begin{aligned}
\widehat{\operatorname{deg}}_{\mathrm{n}} \overline{\mathrm{H}^{0}\left(\mathbb{P}^{1}, \mathcal{O}\left(D_{0}\right)\right)} & =\widehat{\operatorname{deg}}_{\mathrm{n}} \overline{\operatorname{det} \mathrm{H}^{0}\left(\mathbb{P}^{1}, \mathcal{O}\left(D_{0}\right)\right)} \\
& =-\frac{1}{[k: \mathbb{Q}]} \sum_{\sigma: k \rightarrow \mathbb{C}} \log \|s\|_{\operatorname{det} \mathrm{H}^{0}\left(\mathbb{P}^{1}, \mathcal{O}\left(D_{0}\right)\right), \sigma} \\
& =-\log \|s\|_{\operatorname{det} \mathrm{H}^{0}\left(\mathbb{P}_{\mathbb{Z}}^{1}, \mathcal{O}\left(D_{0}\right)\right)}
\end{aligned}
$$

Par définition de la métrique de Fubini-Study et de la métrique induite sur le déterminant, on obtient

$$
\begin{aligned}
\|s\|_{\operatorname{det} H^{0}\left(\mathbb{P}_{\mathbb{Z}}^{1}, \mathcal{O}\left(D_{0}\right)\right)}^{2} & \left.=\operatorname{det}\left(<X_{0}^{h} X_{1}^{D_{0}-h}, X_{0}^{h^{\prime}} X_{1}^{D_{0}-h^{\prime}}\right\rangle\right)_{h, h^{\prime}} \\
& =\prod_{h=0}^{D_{0}}\left\|X_{0}^{h} X_{1}^{D_{0}-h}\right\|^{2} \\
& =\prod_{h=0}^{D_{0}} \frac{h !\left(D_{0}-h\right) !}{\left(D_{0}+1\right) !} \quad(\text { voir formule (5), page 68) }
\end{aligned}
$$

et donc

$$
\widehat{\operatorname{deg}}_{\mathrm{n}} \overline{\mathrm{H}^{0}\left(\mathbb{P}^{1}, \mathcal{O}\left(D_{0}\right)\right)}=\frac{1}{2} \log \prod_{h=0}^{D_{0}} \frac{\left(D_{0}+1\right) !}{h !\left(D_{0}-h\right) !} .
$$

Ce qui clôt la démonstration.

\section{Remarques :}

1. Le fibré hermitien $\overline{\mathscr{E}}$ est semi-stable (au sens de [19], § A.3, page 148). En effet, d'après la proposition A.3 de [19], il suffit de montrer qu'il existe un groupe d'automorphismes isométriques de $\overline{\mathscr{E}}$, qui agisse de façon irréductible sur $\mathscr{E}_{k}$. Or, d'une part, le groupe $\mathrm{U}_{2}(\mathbb{C})$ agit irréductiblement sur $\mathrm{H}^{0}\left(\mathbb{P}_{\mathbb{C}}^{1}, \mathcal{O}\left(D_{0}\right)\right.$ ) (lemme II.3.10) et il existe un groupe (provenant du groupe de MumFord $K(L), c f$. [19], démonstration du théorème 4.2, page 129) qui agit irréductiblement sur $\mathrm{H}^{0}\left(\mathbf{A}, L^{\otimes 3 D_{1}}\right)$ puis, d'autre part, le produit de tels groupes agit irréductiblement sur le produit tensoriel des espaces (lemme II.3.9). Ce qui démontre l'assertion. 


\section{Démonstration du théorème}

2. Nous pouvons donner une formule tout à fait générale pour le degré d'Arakelov de

$$
\mathrm{H}:=\mathrm{H}^{0}\left(\mathbb{P}^{n_{1}} \times \cdots \times \mathbb{P}^{n_{\ell}}, \mathcal{O}\left(d_{1}, \ldots, d_{\ell}\right)\right)
$$

(muni des métriques de Fubini-Study) :

$$
\widehat{\operatorname{deg}}_{\mathrm{n}} \overline{\mathrm{H}}=\frac{\operatorname{dim} \mathrm{H}}{2} \sum_{i=1}^{\ell} \log \left\{\left(\begin{array}{c}
d_{i}+n_{i} \\
n_{i}
\end{array}\right) \Gamma_{i}\right\}
$$

où

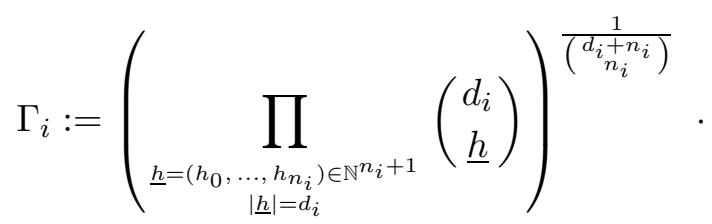

Cette quantité $\Gamma_{i}$ est étudiée en détail dans [51] (voir également lemme 4.2 .1 de [67]).

\section{Choix de la filtration}

La partie analytique de la démonstration du théorème II.2.1 repose sur la méthode de Baker. C'est ce qui détermine le choix de la filtration. À un élément $s \in \mathscr{E}_{k}$, nous associons (via le morphisme $\varphi$ défini ci-après) les jets d'ordre $2(d+1) T($ resp.$(d+1) T)$ le long de $W$ en les points $m$ p pour $m \in\left\{0, \ldots, S_{0}\right\}$ (resp. $m \in\left\{S_{0}+1, \ldots,(d+1) S\right\}$ ). Avec une écriture très imprécise (mais utile pour l'intuition), nous avons

$$
\varphi(s)=\left(\partial_{W}^{\underline{t}} s(m \mathbf{p})\right)
$$

où $(m, \underline{t})$ varie dans

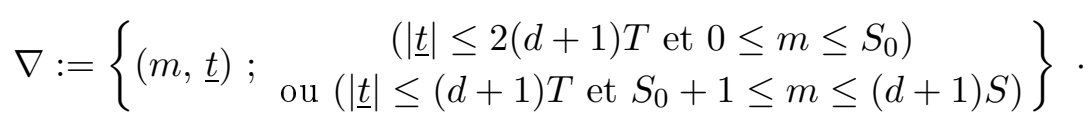

Le $\mathcal{O}_{K}$-module $F$ est construit de manière ad hoc pour « accueillir » ces différents jets. L'ensemble $\nabla$ est muni de l'ordre lexicographique. L'espace vectoriel $F_{K}$ est alors isomorphe à $(m, \underline{t}) \in \nabla$

$\oplus K$. La filtration $\left(F_{i}\right)$ de $F$ est alors simplement définie comme l'annulation des $i$ premières composantes de $F$ :

$$
i=(m, \underline{t}) \text { et } F_{i} \otimes K=\underset{\left(m^{\prime}, \underline{t}^{\prime}\right) \geq(m, \underline{t})}{\oplus} K .
$$

La notion de voisinage infinitésimal va nous permettre de donner un sens mathématique précis à ces considérations.

Étant donné un entier $\ell \in \mathbb{N}$, rappelons tout d'abord (voir appendice C, $\S$ C.2) que $S_{W}^{(\ell)}$ désigne le voisinage infinitésimal d'ordre $\ell$ le long de $W$ (en la section nulle de $\mathbf{G}$ ). C'est un sous-schéma fermé de G. Pour $m \in\{0, \ldots,(d+1) S\}$, notons $S_{W, m \mathbf{p}}^{(\ell)}$ le sous-schéma fermé de G obtenu à partir de $S_{W}^{(\ell)}$ en translatant par $m \mathbf{p}$, et notons $\overline{S_{W, m \mathbf{p}}^{(\ell)}}$ l'adhérence schématique de $S_{W, m \mathbf{p}}^{(\ell)}$ dans $\mathscr{X}$. Considérons $F$ le $\mathcal{O}_{k}$-module :

$$
F:=\underset{m=0}{\bigoplus_{0}} \pi_{*} \mathscr{M}\left|\frac{}{S_{W, m \mathbf{p}}^{(2(d+1) T)}} \bigoplus \underset{m=S_{0}+1}{\stackrel{(d+1) S}{\bigoplus}} \pi_{*} \mathscr{M}\right| \frac{\mid S_{W, m \mathbf{p}}^{((d+1) T)}}{}
$$


où

$$
\mathscr{M} \mid \overline{S_{W, m \mathbf{p}}^{(\ell)}}
$$

désigne la restriction du fibré $\mathscr{M}$ au sous-schéma fermé $\overline{S_{W, m \mathbf{p}}^{(\ell)}}$ de $\mathscr{X}$ et $\varphi: \mathscr{E} \rightarrow F$ le morphisme de restriction. D'après le choix des paramètres et la proposition II.5.1, ce morphisme est injectif. Soit $\mathcal{K}_{m}^{(\ell)}$ le faisceau d'idéaux définissant $\overline{S_{W, m \mathbf{p}}^{(\ell)}}$ dans $\overline{S_{W, m \mathbf{p}}^{(2(d+1) T)}}$ (resp. dans $\overline{S_{W, m \mathbf{p}}^{((d+1) T)}}$ ) lorsque $m \in\left\{0, \ldots, S_{0}\right\}$ (resp. $m \in\left\{S_{0}+1, \ldots,(d+1) S\right\}$ ). Notons que le paramètre $T$, l'hyperplan $W$ et le point $\mathbf{p}$ sont sous-entendus dans la définition de $\mathcal{K}_{m}^{(\ell)}$; ces données étant fixées, il n'y aura aucune confusion possible. On a $\mathcal{K}_{m}^{(2(d+1) T)}=\{0\}$ si $m \in\left\{0, \ldots, S_{0}\right\}$ et $\mathcal{K}_{m}^{((d+1) T)}=\{0\}$ si $m \in\left\{S_{0}+1, \ldots,(d+1) S\right\}$.

Pour $m \in\left\{0, \ldots, S_{0}\right\}$ et $\ell \in\{0, \ldots, 2(d+1) T\}$, considérons le $\mathcal{O}_{K}$-module

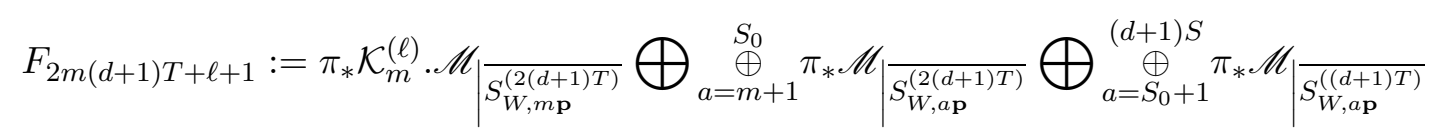

et, pour $m \in\left\{S_{0}+1, \ldots,(d+1) S\right\}$ et $\ell \in\{0, \ldots,(d+1) T\}$, nous posons

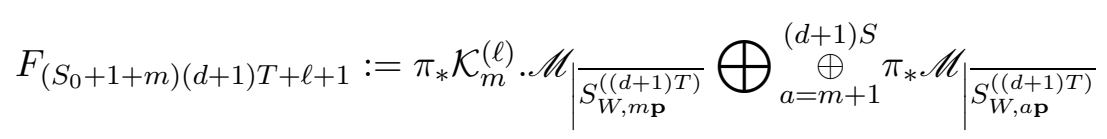

$\left(F_{2\left(S_{0}+1\right)(d+1) T+1}\right.$ est défini deux fois ... pareillement !) Soit $\mathcal{N}:=\left(S_{0}+2+(d+1) S\right)(d+1) T+1$ le nombre de pas de la filtration :

$$
\{0\}=F_{\mathcal{N}} \subseteq F_{\mathcal{N}-1} \subseteq \cdots \subseteq F_{1} \subseteq F_{0}:=F .
$$

En considérant $i=2 m(d+1) T+\ell$ ou $\left(S_{0}+1+m\right)(d+1) T+\ell$ selon que $m \leq S_{0}$ ou $m>S_{0}$ (nous dirons alors que le couple $(\ell, m)$ est associé à $i$ ), le quotient

$$
G_{i}:=F_{i} / F_{i+1}=\operatorname{ker}\left(\pi_{*} \mathscr{M}\left|\frac{}{S_{W, m \mathbf{p}}^{(\ell)}} \longrightarrow \pi_{*} \mathscr{M}\right| \frac{}{S_{W, m \mathbf{p}}^{(\ell+1)}}\right)
$$

est sans torsion. De plus le $\mathcal{O}_{K}$-module $G_{i}^{\prime}:=S^{\ell} \mathcal{W}^{\vee} \otimes \varepsilon_{m \mathbf{p}}^{*} \mathscr{M}$ (où $\mathcal{W}=W \cap t_{\mathscr{G}_{\mathcal{O}_{K}}}$ ) s'injecte naturellement dans $G_{i}$ (par définition de l'image schématique, $\overline{S_{W, m p}^{(\ell)}}$ est un sous-schéma fermé de $S_{\mathcal{W}}^{(\ell)}=\operatorname{Spec}\left(\mathcal{O}_{K} \oplus \mathcal{W} \oplus \cdots \oplus S^{\ell} \mathcal{W}\right)$, cf. appendice C, pour plus de détails), et les $K$-espaces vectoriels $G_{i}^{\prime} \otimes K$ et $G_{i} \otimes K$ sont isomorphes. Malheureusement, en toute généralité, l'injection $G_{i}^{\prime} \hookrightarrow G_{i}$ n'est pas un isomorphisme (sur $\mathcal{O}_{K}$ ) et le conoyau de cette application est de cardinal * $\leq \ell$ ! Dans le cas particulier où $\mathcal{W}$ est l'espace tangent d'un sous-groupe lisse de $\mathscr{G}$ (ce qui est impossible ici pour nous $!^{\dagger}$ ), les modules $G_{i}^{\prime}$ et $G_{i}$ sont isomorphes (pour tout $i$ ). Nous aurons

*Signalons à ce propos une erreur dans [19], où la torsion du conoyau était négligée et, en l'occurrence, cela n'avait aucune conséquence importante sur la démonstration ; seules quelques constantes devaient être revues à la hausse. Cette erreur a été corrigée ultérieurement par l'auteur lui-même (J.-B. Bost) et ce n'est qu'après avoir obtenu une 《 merveilleuse » (et néanmoins réaliste) mesure d'indépendance linéaire, où tous les logarithmes « en trop» des hauteurs du point $\mathbf{p}$ et de l'hyperplan $W$ disparaissaient, que je prenais conscience de cette erreur...

${ }^{\dagger}$ Le procédé de réduction de N. Hirata-Kohno, que nous utilisons ici et qui positionne l'hyperplan $\mathcal{W}$ transversalement à $t_{\mathbb{G}_{a}}$, est la cause de cette difficulté. 


\section{Démonstration du théorème}

l'occasion de donner un exemple où ces deux modules sont isomorphes au $\S$ II.5.d. Le lemme suivant est un lemme clef pour obtenir une minoration de $|\Lambda|$ optimale en la hauteur $h(W)$ de l'hyperplan. Il est de nature arithmétique.

Lemme II.5.4. Notons, comme dans la première partie (voir définition 1.14.1, p. 37), pour $\ell, h$ des entiers naturels non nuls,

$$
\delta_{\ell}(h)=\operatorname{ppcm}\left\{i_{1} \ldots i_{h^{\prime}} ; \quad 1 \leq h^{\prime} \leq h, i_{j} \in \mathbb{N}^{*}, \quad i_{1}+\cdots+i_{h^{\prime}} \leq \ell\right\} .
$$

Alors, pour tout $i \in\{0, \ldots, \mathcal{N}-1\}$, on a $\delta_{\ell}\left(D_{0}\right) \cdot G_{i} \subseteq G_{i}^{\prime}$ où $\ell$ est l'entier correspondant à $i$, dans la construction ci-dessus.

Démonstration. La preuve de ce lemme repose sur les mêmes arguments que celle du lemme I.14.5 et elle se simplifie légèrement en évitant le recours aux formules d'additions sur la variété abélienne A.

Nous allons montrer que, pour tout idéal $\mathfrak{P} \subseteq \mathcal{O}_{K}$, d'anneau de valuation $\mathcal{O}_{\mathfrak{P}}$, on a l'inclusion $\delta_{\ell}\left(D_{0}\right) \cdot G_{i} \otimes_{\mathcal{O}_{K}} \mathcal{O}_{\mathfrak{P}} \subseteq G_{i}^{\prime} \otimes_{\mathcal{O}_{K}} \mathcal{O}_{\mathfrak{P}}$.

Soit

$$
\mathscr{X}_{\mathfrak{P}}:=\mathscr{X} \underset{\operatorname{Spec} \mathcal{O}_{K}}{\times} \operatorname{Spec} \mathcal{O}_{\mathfrak{P}}
$$

et, pour l'entier $m \in\{0, \ldots,(d+1) S\}$ associé à $i$, désignons par $\widehat{\mathscr{X}}_{\mathfrak{P}, m}$ le complété formel de $\mathscr{X}_{\mathfrak{P}}$ (ou de $\mathscr{G} \times \operatorname{Spec} \mathcal{O}_{\mathfrak{P}}$, ce qui est la même chose) le long de (l'idéal de définition) de $\varepsilon_{m \mathbf{p}} \in \mathscr{X}\left(\mathcal{O}_{K}\right) \subseteq \mathscr{X}_{\mathfrak{P}}\left(\mathcal{O}_{\mathfrak{P}}\right)$. Le schéma formel $\widehat{\mathscr{X}}_{\mathfrak{P}, m}$ est le translaté par $\varepsilon_{m \mathbf{p}}$ du groupe formel $\widehat{\mathscr{X}_{\mathfrak{P}, 0}}$ sur Spec $\mathcal{O}_{\mathfrak{P}}$. On se ramène ainsi à $m=0$. Comme $\mathscr{X}_{\mathfrak{P}} \rightarrow \operatorname{Spec} \mathcal{O}_{\mathfrak{P}}$ est lisse et $\mathcal{O}_{\mathfrak{P}}$ est un anneau principal, nous disposons (proposition I.5.2, première partie) d'un isomorphisme (de schémas formels)

$$
\widehat{\mathscr{X}}_{\mathfrak{P}, 0} \stackrel{\sim}{\longrightarrow} \operatorname{Specf} \mathcal{O}_{\mathfrak{P}}\left[\left[X_{0}, X_{1}, \ldots, X_{d}\right]\right]
$$

compatible avec le scindage $\mathbb{G}_{\mathrm{a}} \times \mathscr{A}$ (ainsi $\left.\widehat{\mathbb{G}}_{\mathrm{a}, \mathfrak{P}}=\operatorname{Specf} \mathcal{O}_{\mathfrak{P}}\left[\left[X_{0}\right]\right]\right)$. La structure de groupe formel de $\widehat{\mathscr{X}_{\mathfrak{P}, m}}$ se transporte alors sur $\operatorname{Specf} \mathcal{O}_{\mathfrak{P}}\left[\left[X_{0}, X_{1}, \ldots, X_{d}\right]\right]$. Notons

$$
\ell\left(X_{0}, \ldots, X_{d}\right)=\left(X_{0}, \ell_{1}\left(X_{1}, \ldots, X_{d}\right), \ldots, \ell_{d}\left(X_{1}, \ldots, X_{d}\right)\right) \in K_{\mathfrak{P}}\left[\left[X_{0}, \ldots, X_{d}\right]\right]^{d+1}
$$

le logarithme formel de $\widehat{\mathscr{X}_{\mathfrak{P}, m}}$ (relatif à la base $\left(e_{0}, \ldots, e_{d}\right)$ de $\left.t_{\mathscr{G}}\right)$. L'homorphisme canonique

$$
\nu: \Gamma\left(\mathscr{X}_{\mathfrak{P}}, \mathscr{M}\right) \otimes \mathcal{O}_{\mathfrak{P}} \rightarrow \Gamma\left(\widehat{\mathscr{X}_{\mathfrak{P}, m}}, \widehat{\mathscr{M}_{\mathfrak{P}}}\right) \simeq \mathcal{O}_{\mathfrak{P}}\left[\left[X_{0}, \ldots, X_{d}\right]\right]
$$

associe à une section $s \in \Gamma(\mathscr{X}, \mathscr{M}) \otimes \mathcal{O}_{\mathfrak{P}}$ une série formelle

$$
\nu(s)=\sum_{\substack{i \in \mathbb{N}^{d}+1 \\ 0 \leq i_{0} \leq D_{0}}} \theta_{\underline{i}} \cdot X_{0}^{i_{0}} \cdots X_{d}^{i_{d}} .
$$

Le point est que $s$ s'annule le long de $W$ en $m$ p à l'ordre $\ell$ si et seulement si

$$
\nu(s)\left(\beta_{1} \ell_{1}+\cdots+\beta_{d} \ell_{d}, X_{1}, \ldots, X_{d}\right) \in\left(X_{1}, \ldots, X_{d}\right)^{\ell},
$$

car si $z_{0}, \ldots, z_{d}$ désignent les coordonnées sur l'espace tangent $t_{\mathbf{G}}$, les idéaux $\left(z_{0}, \ldots, z_{d}\right)^{h}$ et $\left(X_{0}, \ldots, X_{d}\right)^{h}$ sont égaux $\left(z_{i}=\ell_{i}\right)$. La structure même des coefficients du logarithme formel 
(qui provient de l'intégration d'une forme différentielle à coefficients dans $\mathcal{O}_{\mathfrak{P}}$, voir appendice $\mathrm{C})$ implique que l'unique polynôme homogène de degré $\ell$ qui représente $\delta_{\ell}\left(D_{0}\right) \cdot \nu(s)\left(\beta_{1} \ell_{1}+\right.$ $\left.\cdots+\beta_{d} \ell_{d}, X_{1}, \ldots, X_{d}\right)$ dans $\left(X_{1}, \ldots, X_{d}\right)^{\ell} /\left(X_{1}, \ldots, X_{d}\right)^{\ell+1}$ est à coefficients dans $\mathcal{O}_{\mathfrak{P}}$, ce qui signifie que le jet de $\delta_{\ell}\left(D_{0}\right) \cdot s$, d'ordre $\ell$ au point $m \mathbf{p}$, appartient à $\varepsilon_{m \mathbf{p}}^{*} \mathscr{M} \otimes \mathcal{O}_{\mathfrak{P}}$, i.e.

$$
\delta_{\ell}\left(D_{0}\right) \cdot G_{i} \otimes \mathcal{O}_{\mathfrak{P}} \subseteq G_{i}^{\prime} \otimes \mathcal{O}_{\mathfrak{P}}
$$

Ce lemme contient les estimations des normes ultramétriques ( i.e. la partie finie de la hauteur) du morphisme

$$
\varphi_{i}: \mathscr{E}_{i} \rightarrow G_{i}
$$

déduit de $\varphi$, où $\mathscr{E}_{i}:=\varphi^{-1}\left(F_{i}\right)$.

\section{$\S$ 5.b. Pente maximale des fibrés hermitiens quotients}

Soit $\mathcal{P}: t_{\mathscr{X}_{\mathcal{O}_{K}}} \rightarrow \mathcal{W}$ la projection sur $\mathcal{W}$ parallèlement à $t_{\mathbb{G}_{\mathrm{a}}}:$

$$
\mathcal{P}\left(x_{0} e_{0}+\cdots+x_{d} e_{d}\right)=x_{1} \widetilde{e}_{1}+\cdots+x_{d} \widetilde{e}_{d}
$$

où $\widetilde{e}_{i}=e_{i}+\beta_{i} e_{0} \in \mathcal{W}$ (cela est possible car $\beta_{i} \in \mathcal{O}_{K}$, voir remarque précédent le $\S$ II.5.a). Notons, pour $\ell \in \mathbb{N}^{*}, \mathcal{P}_{\ell}^{\vee}: S^{\ell} \mathcal{W}^{\vee} \rightarrow S^{\ell} t_{\mathscr{X}_{\mathcal{O}_{K}}}$ l'injection induite par $\mathcal{P}$. Nous conférons au $\mathcal{O}_{k^{-}}$ module $t_{\mathscr{X}}=t_{\mathscr{G}}$ la structure de fibré hermitien décrite au $\S$ II.3.b., qui se transporte à $S^{\ell} t_{\mathscr{X}}^{v}$, comme expliqué au $\S$ II.3.b. . De l'égalité (4.5) et de l'inégalité (4.6) de [22], nous déduisons :

$$
\begin{aligned}
\widehat{\mu}_{\max }\left(\overline{S^{\ell} t_{\mathscr{C}_{\mathcal{O}_{K}}} \otimes_{\mathcal{O}_{k}} \varepsilon_{m \mathbf{p}}^{*} \mathscr{M}}\right) & =\widehat{\mu}_{\max }\left(\overline{S^{\ell} t_{\mathscr{X}_{\mathcal{O}_{K}}^{v}}}\right)+\widehat{\operatorname{deg}}_{\mathrm{n}} \overline{\varepsilon_{m \mathbf{p}}^{*} \mathscr{M}} \\
& \leq \ell\left(\widehat{\mu}_{\max }\left(\overline{t_{\mathscr{X}}^{v}}\right)+2(d+1) \log (d+1)\right)+D_{0} \cdot h \overline{\mathcal{O}(1)}\left(m u_{0}\right)+3 D_{1} \cdot \widehat{h}(m \mathbf{q})
\end{aligned}
$$

où $\widehat{h}$ est la hauteur de Néron-Tate relative à $(\mathbf{A}, L)$ (cf. ii $)$ du théorème II.3.2). De plus $\overline{t_{\mathscr{X}}^{v}}=\overline{\omega_{\mathbb{G}_{\mathrm{a}} \mid \mathcal{O}_{k}}^{1}} \oplus \overline{\omega_{\mathscr{A} \mid \mathcal{O}_{k}}^{1}}$ donc

$$
\begin{aligned}
\widehat{\mu}_{\max }\left(\overline{t_{\mathscr{X}}^{v}}\right) & =\max \left\{\widehat{\mu}_{\max }\left(\overline{\omega_{\mathbb{G}_{a} \mid \mathcal{O}_{k}}^{1}}\right), \widehat{\mu}_{\max }\left(\overline{\omega_{\mathscr{A} \mid \mathcal{O}_{k}}^{1}}\right)\right\} \\
& =\widehat{\mu}_{\max }\left(\overline{\omega_{\mathscr{A} \mid \mathcal{O}_{k}}^{1}}\right)^{+} .
\end{aligned}
$$

Avec le lemme II.3.11 et la proposition 2.14 de [39], nous déduisons alors le

Lemme II.5.5. Pour tout $\ell \in\{0, \ldots, 2(d+1) T\}$ et $m \in\{0, \ldots,(d+1) S\}$, on a

$$
\begin{aligned}
& \widehat{\mu}_{\max }\left(\overline{S^{\ell} t_{\mathscr{X}_{\mathcal{O}_{K}}} \otimes_{\mathcal{O}_{k}} \varepsilon_{m \mathbf{p}}^{*} \mathscr{M}}\right) \\
& \leq 2(d+1) T\left((d+1) h_{F}(\mathbf{A})+10 d^{5}\right)+D_{0}\left(\log (\sqrt{2}(d+1) S)+h_{\text {Weil }}\left(u_{0}\right)\right)+3 D_{1}(d+1)^{2} S^{2} \widehat{h}(\mathbf{q}) .
\end{aligned}
$$

Le terme de droite de l'inégalité apporte la contribution arithmétique dans l'inégalité de pentes. La partie analytique apparaîtra au moment de l'évaluation des normes du morphisme $\widetilde{\varphi}_{i}$ qui sera attaché à $\varphi_{i}$. 


\section{$\S$ 5.c. Rang d'un sous-fibré}

Lemme II.5.6. Soit $i_{0}:=2\left(S_{0}+1\right)(d+1) T+1$. Si

$$
S / S_{0} \geq 8^{d+1}(d+1)^{d+1} C_{0}
$$

alors

$$
\operatorname{rg} \mathscr{E}_{i_{0}} \geq \frac{\operatorname{rg} \mathscr{E}}{2} .
$$

Démonstration. C'est l'analogue du lemme I.11.1 (page 28) de la première partie de la thèse. Comme nous sommes dans le cas « non-périodique », la démonstration repose sur le choix de $S_{0}$. En adaptant la preuve du lemme 6.1 de [30], on montre que

$$
\operatorname{rg} \mathscr{E}_{i_{0}} \geq\left(1-\frac{8^{d}(d+1)^{\widetilde{r}+1} C_{0} S_{0}}{S}\right) \times \operatorname{rg} \mathscr{E}
$$

\section{$\S$ 5.d. Entracte}

Ce paragraphe est totalement indépendant de la démonstration du théorème II.2.1 (mais les notations restent cohérentes).

Dans un soucis didactique, nous allons détailler le lien qui unit $G_{i}$ et $G_{i}^{\prime}$ (§ II.5.a.) dans le cas où le groupe algébrique est $\mathbb{G}_{\mathrm{m}}^{d}(\operatorname{sur} \mathbb{Z})$ et $\mathcal{W}$ est l'espace tangent de

$$
\begin{aligned}
\mathbf{G}^{\prime} & :=D_{\mathbb{Z}}\left(\mathbb{Z}^{d} /\left(a_{1}, \ldots, a_{d-1}, 1\right) \cdot \mathbb{Z}\right) \\
& =\left\{\left(x_{1}, \ldots, x_{d}\right) ; x_{1}^{a_{1}} \cdots x_{d-1}^{a_{d-1}}=x_{d}\right\}
\end{aligned}
$$

avec $a_{i} \in \mathbb{Z}$. Le point $\mathbf{p}$ ne jouant aucun rôle particulier, nous prendrons $1_{\mathbb{G}_{\mathrm{m}}^{d}}$ (i.e. la section nulle $\varepsilon$ ). Le groupe $\mathbf{G}^{\prime}$ est lisse sur $\mathbb{Z}$ (voir le corollaire C.4.9 de l'appendice C). On plonge $\mathbb{G}_{\mathrm{m}}^{d}$ dans $\mathbb{P}:=\left(\mathbb{P}^{1}\right)^{d}$ via le plongement usuel de $\mathbb{G}_{\mathrm{m}}$ dans $\mathbb{P}^{1}$ et $\mathscr{M}=\mathcal{O}_{\mathbb{P}}\left(D_{1}, \ldots, D_{d}\right)$ est le faisceau dont les sections globales sont les polynômes multihomogènes en $\left(X_{0}^{(i)}, X_{1}^{(i)}\right)_{i=1, \ldots, d}$, de multidegré $\left(D_{1}, \ldots, D_{d}\right)$. Les sections de $\mathbb{G}_{\mathrm{m}}^{d}$ sur $\mathbb{Z}$ s'identifient alors aux fonctions holomorphes $P\left(e^{z_{1}}, \cdots, e^{z_{d}}\right)$, où $P$ est un polynôme à coefficients entiers et de degré partiel par rapport à $X_{i}$ inférieur ou égal à $D_{i}$. Notons, pour $\ell \in \mathbb{N}, \mathscr{Q}_{\ell}$ l'ensemble

$$
\left\{s\left(\leftrightarrow P\left(e^{z_{1}}, \cdots, e^{z_{d}}\right)\right) \in \Gamma\left(t_{\mathbb{G}_{\mathrm{m}}^{d}}(\mathbb{C}), \exp ^{*} \mathcal{O}_{\mathbb{G}_{\mathrm{m}}^{d}}\right) ; \prod_{j=1}^{d-1}\left(\frac{\partial}{\partial z_{j}}+a_{j} \frac{\partial}{\partial z_{d}}\right)^{i_{j}} P\left(e^{z_{1}}, \cdots, e^{z_{d}}\right)(0)=0 \text { pour }|\underline{i}|<\ell\right\}
$$

et $R$ le $\mathbb{Z}$-module

$$
\operatorname{Vect}_{\mathbb{Z}}\left(\frac{1}{\underline{i} !} \prod_{j=1}^{d-1}\left(\frac{\partial}{\partial z_{j}}+a_{j} \frac{\partial}{\partial z_{d}}\right)^{i_{j}} P\left(e^{z_{1}}, \cdots, e^{z_{d}}\right)(0)\right)_{\substack{|i|=\ell \\ P \in \mathscr{Q} \ell}} .
$$

Alors $G_{i}^{\prime}=S^{\ell} \mathcal{W}^{\vee} \otimes \varepsilon^{*} \mathscr{M}$ et $G_{i}=S^{\ell} \mathcal{W}^{\vee} \otimes \varepsilon^{*} \mathscr{M} \otimes_{\mathbb{Z}} R$. Nous allons montrer que $R=\mathbb{Z}$. Pour cela, considérons

$$
\Delta_{n}(X)=\frac{X(X-1) \cdots(X-n+1)}{n !},
$$

le $n^{\text {ième }}$ polynôme de Fel'dman $\left(\Delta_{0}(X)=1\right.$ par convention). Soit $\left(p_{\underline{\alpha}}\right)_{\underline{\alpha}}$ les coefficients d'un polynôme $P$ comme ci-dessus. Pour $\underline{i}=\left(i_{1}, \ldots, i_{d-1}\right) \in \mathbb{N}^{d-1}$, nous noterons

$$
Q_{\underline{i}}\left(X_{1}, \ldots, X_{d}\right):=\sum_{\underline{\alpha}} p_{\underline{\alpha}} \cdot \Delta_{i_{1}}\left(\alpha_{1}+\alpha_{d} a_{1}\right) \cdots \Delta_{i_{d-1}}\left(\alpha_{d-1}+\alpha_{d} a_{d-1}\right) \cdot X_{1}^{\alpha_{1}} \cdots X_{d}^{\alpha_{d}} .
$$


Alors, pour tout $\underline{i} \in \mathbb{N}^{d-1}$, il existe des nombres rationnels $\mu_{\underline{j}, \underline{i}}(|\underline{j}|<|\underline{i}|)$ tels que

$$
\prod_{j=1}^{d-1}\left(\frac{\partial}{\partial z_{j}}+a_{j} \frac{\partial}{\partial z_{d}}\right)^{i_{j}} P\left(e^{z_{1}}, \cdots, e^{z_{d}}\right)(0)
$$

soit égal à

$$
i_{1} ! \cdots i_{d-1} ! \cdot Q_{\underline{i}}(1, \ldots, 1)+\sum_{|\underline{j}|<|\underline{i}|} \mu_{\underline{j}, \underline{i}} \prod_{h=1}^{d-1}\left(\frac{\partial}{\partial z_{h}}+a_{h} \frac{\partial}{\partial z_{d}}\right)^{j_{h}} P\left(e^{z_{1}}, \cdots, e^{z_{d}}\right)(0)
$$

En particulier, lorsque les dérivées d'ordre $<|\underline{i}|=\ell$ de $P\left(e^{z_{1}}, \ldots, e^{z_{d}}\right)$ (en 0 et le long de $\mathcal{W}$ ) sont nulles $($ ce qui est le cas dans le quotient $G_{i}$ ), le coefficient de Taylor

$$
\frac{1}{i_{1} ! \cdots i_{d-1} !} \cdot \prod_{j=1}^{d-1}\left(\frac{\partial}{\partial z_{j}}+a_{j} \frac{\partial}{\partial z_{d}}\right)^{i_{j}} P\left(e^{z_{1}}, \cdots, e^{z_{d}}\right)(0)
$$

est un entier relatif. La démonstration (élémentaire) de l'égalité de (33) et de (34) consiste à écrire $X^{n}$ dans la base $\left\{1, X, \ldots, X^{n-1}, \Delta_{n}(X)\right\}$ :

$$
X^{n}=n ! \Delta_{n}(X)+\mu_{n-1} X^{n-1}+\cdots+\mu_{0}
$$

Le fait que le coefficient de Taylor (35) soit un entier découle aussi immédiatement du théorème C.5.6 de l'appendice $\mathrm{C}$ appliqué à $\mathbf{G}^{\prime} \rightarrow$ Spec $\mathbb{Z}$, qui est un morphisme lisse donc différentiellement lisse. En conclusion, ce lien entre dérivées divisées et polynômes de Fel'dman explique pourquoi les mesures d'indépendances les plus précises connues à l'heure actuelle concernent le cas rationnel homogène.

\section{$\S$ 5.e. Reprise analytique}

Rappelons que $\varphi_{i}: \mathscr{E}_{i} \rightarrow G_{i}$ désigne le morphisme déduit de $\varphi$ et posons

$$
\widetilde{\varphi}_{i}:=\mathcal{P}_{\ell}^{v} \circ\left(\delta_{\ell}\left(D_{0}\right) \cdot \varphi_{i}\right): \mathscr{E}_{i} \rightarrow S^{\ell} t_{\mathscr{X}_{K}}^{v} \otimes_{\mathcal{O}_{k}} \varepsilon_{m \mathbf{p}}^{*} \mathscr{M} .
$$

C'est un morphisme de $\mathcal{O}_{K}$-modules. Nous allons appliquer l'inégalité de pentes (proposition 4.4 de [19]) à

$$
\begin{gathered}
\left(\left(\mathscr{E}_{i}\right)_{i},\left(S^{\ell} t_{\mathscr{C}_{\mathcal{O}_{K}}^{v}} \otimes \varepsilon_{m \mathrm{p}}^{*} \mathscr{M}\right)_{(\ell, m)},\left(\widetilde{\varphi}_{i}\right)_{i}\right): \\
\widehat{\operatorname{deg}}_{\mathrm{n}} \overline{\mathscr{E}} \leq \sum_{i=0}^{\mathcal{N}-1}\left(\operatorname{rg} \mathscr{E}_{i}-\operatorname{rg} \mathscr{E}_{i+1}\right)\left(\widehat{\mu}_{\max }\left({\overline{S^{\ell}}}_{\mathscr{\mathscr { T }}_{\mathscr{O}_{K}}^{\mathrm{v}}} \otimes \varepsilon_{m \mathrm{p}}^{*} \mathscr{M}\right)+\frac{1}{[K: \mathbb{Q}]} \sum_{\sigma: K \rightarrow \mathbb{C}} \log \left\|\widetilde{\varphi}_{i}\right\|_{\sigma}\right)
\end{gathered}
$$

où $\|\cdot\|_{\sigma}$ est la norme d'opérateur de $\widetilde{\varphi}_{\underline{\mathscr{C}}}$ (nous l'examinerons en détail au paragraphe suivant). L'estimation du degré d'Arakelov de $\mathscr{E}$ a été l'objet de la proposition II.5.3 et la pente maximale de $\overline{S^{\ell} t_{\mathscr{K}}^{v} \otimes \varepsilon_{m \mathbf{p}}^{*} \mathscr{M}}$ a été majorée au lemme II.5.5. Il ne reste donc qu'à évaluer les normes archimédiennes des morphismes $\widetilde{\varphi}_{i}$.

Remarque : nous avons composé $\varphi_{i}$ avec $\mathcal{P}_{\ell}^{v}$ (pour définir $\widetilde{\varphi}_{i}$ ) afin d'éviter que ce ne soit la pente maximale de $\overline{S^{\ell} \mathcal{W}^{\vee} \otimes \varepsilon_{m p}^{*} \mathscr{M}}$ qui intervienne dans l'inégalité (37). En effet, si $\mathcal{W}$ est muni des métriques induites par celles de $t_{\mathscr{X}}$, la pente maximale d'un tel fibré contient le terme $T \cdot h(W)$ (où $h(W)$ est une hauteur de l'hyperplan), d'après le lemme 5.7 de [19]. Comme le paramètre $T$ est proportionnel à $h(W)$, on ne pourrait alors espérer obtenir une 
mesure d'indépendance linéaire optimale (i.e. linéaire) en $h(W)$. Le coût de ce subterfuge est des estimations archimédiennes plus délicates et un terme

$$
\frac{1}{[K: \mathbb{Q}]} \sum_{\sigma: K \rightarrow \mathbb{C}} \log \frac{1}{\mathrm{~d}_{\sigma}\left(e_{0}, W_{\sigma}\right)}
$$

de la mesure d'indépendance (de l'ordre de $h(W)$ ) que je ne sais pas expliciter, sans avoir recours à une base de l'espace tangent $t_{\mathbf{G}}$, i.e. sans accepter de perdre un peu du caractère intrinsèque de la mesure ...

\section{$\S$ 5.f. Hauteur des morphismes}

\section{Évaluation simple des $\left\|\widetilde{\varphi}_{i}\right\|_{\sigma}$}

Après une description précise des morphismes $\widetilde{\varphi}_{i} \otimes_{\sigma} \mathbb{C}$ et des normes d'opérateur $\|\cdot\|_{\sigma}$ associées, la principale difficulté sera de contrôler les termes agissant sur la hauteur de l'hyperplan, en évitant soigneusement de faire apparaître la puissance $\ell^{i e ̀ m e}$ du maximum de 1 et des $\left|\sigma\left(\beta_{i}\right)\right|$ $(i=1, \ldots, d)$.

Soient $\sigma: K \rightarrow \mathbb{C}$ une place archimédienne de $K$ et $s \in \mathscr{E}_{i, \sigma}$. Le choix d'une trivialisation $s_{0}$ de $\mathscr{M}_{\sigma}$ au-dessus d'un ouvert $U$ contenant $m \sigma(\mathbf{p})$ détermine une unique fonction holomorphe $F_{s}\left(z_{0}, \ldots, z_{d}\right)$, correspondant à $s$ (voir $\S$ C.2.c de l'appendice $\mathrm{C}$ ); de plus, si nous notons $\mathbf{u}_{\sigma}$ un logarithme de $\sigma(\mathbf{p})$, dont la projection sur $t_{\mathbf{A}_{\sigma}}(\mathbb{C})$ est de norme inférieure à $2 \sqrt{d}$ (c'est possible d'après le théorème de Minkowski), la fonction $F_{s}$ s'annule en $\mathbf{u}_{\sigma}$ le long de $W_{\sigma}$ à l'ordre $\ell$ (pour $s \in \mathscr{E}_{i}$ ). Localement, on a donc

$$
s(x)=F_{s}(z) \cdot s_{0}(x)
$$

où $x=\exp _{\mathbf{G}_{\sigma}(\mathbb{C})}(z)$. Par définition,

$$
\widetilde{\varphi}_{i}(s) \in S^{\ell} t_{\mathscr{X}_{\sigma}}(\mathbb{C})^{\mathrm{v}} \otimes \varepsilon_{m \sigma(\mathbf{p})}^{*} \mathscr{M}=\operatorname{Hom}_{\mathbb{C}}\left(S^{\ell} t_{\mathscr{X}_{\sigma}}(\mathbb{C}), \varepsilon_{m \sigma(\mathbf{p})}^{*} \mathscr{M}\right)
$$

est le jet d'ordre $\ell$ tronqué le long de $W_{\sigma}$ en $m \sigma(\mathbf{p})$. Soit $\left(b_{0}, \ldots, b_{d}\right)$ une base orthonormée de $t_{\mathscr{X}_{\sigma}}(\mathbb{C})=t_{\mathbb{G}_{\mathrm{a}}}(\mathbb{C}) \stackrel{\perp}{\oplus} t_{\mathbf{A}_{\sigma}}(\mathbb{C})$. Si $\mathcal{D} \in S^{\ell} t_{\mathscr{X}_{\sigma}}(\mathbb{C})$ s'écrit $^{\prime}$

$$
\sum_{|\underline{i}|=\ell} a_{\underline{i}} \cdot b_{0}^{i_{0}} \cdots b_{d}^{i_{d}}
$$

alors, par définition de la métrique quotient sur $S^{\ell} t_{\mathscr{X}_{\sigma}}(\mathbb{C})$ (voir $\S$ II.3.b.), on a

$$
\|\mathcal{D}\|_{S^{\ell} t_{\mathscr{X}_{\sigma}(\mathbb{C})}^{2}}=\sum_{|\underline{i}|=\ell}\left|a_{\underline{i}}\right|^{2} \times \frac{\underline{i} !}{\ell !} .
$$

$\mathrm{Si}$, maintenant $\mathrm{b}_{0}$ est une base orthonormée de $t_{\mathbb{G}_{\mathrm{a}}}(\mathbb{C}),\left(\mathrm{b}_{1}, \ldots, \mathrm{b}_{d}\right)$ une base orthonormée de $W_{\sigma}$ et $\mathcal{D}=\sum a_{\underline{i}} \underline{\underline{i}}^{\underline{i}}$ alors

$$
\mathcal{P}_{\ell}(\mathcal{D})=\sum_{\substack{i \in \mathbb{N}^{d} \\|\underline{|i|}|=\ell}} a_{(0, \underline{i})} \mathrm{b}_{1}^{i_{1}} \cdots \mathrm{b}_{d}^{i_{d}}
$$


et

$$
\widetilde{\varphi}_{i}(s)(\mathcal{D})=\delta_{\ell}\left(D_{0}\right)\left(\sum_{|\underline{i}|=\ell} a_{(0, \underline{i})} \cdot \frac{\left(\frac{\partial}{\partial z_{1}}\right)^{i_{1}} \cdots\left(\frac{\partial}{\partial z_{d}}\right)^{i_{d}}}{i_{1} ! \cdots i_{d} !} F_{s}\left(m \mathbf{u}_{\sigma}+z_{1} \mathrm{~b}_{1}+\cdots+z_{d} \mathrm{~b}_{d}\right)(0)\right) \cdot s_{0}(m \sigma(\mathbf{p})) .
$$

Notons que le membre de droite de cette égalité est bien indépendant à la fois de la trivialisation $s_{0}$ choisie car $s \in \mathscr{E}_{i, \sigma}$ (les dérivées le long de $W_{\sigma}$ d'ordre $<\ell$ sont nulles) et du choix de la base $b_{1}, \ldots, b_{d}$ de $W_{\sigma}$. Choisissons la trivialisation $s_{0}$ pour laquelle

$$
\left\|s_{0}(m \sigma(\mathbf{p}))\right\|_{\varepsilon_{m \mathbf{p}}^{*} \mathscr{M}, \sigma}=\frac{1}{\left(1+\left|m \sigma\left(u_{0}\right)\right|^{2}\right)^{D_{0} / 2}} \times e^{-\frac{\pi}{2} D_{1} m^{2}\left\|\mathbf{u}_{\sigma}^{\prime}\right\|_{L, \sigma}^{2}} \quad \text { (voir équation (6)) }
$$

où $\mathbf{u}_{\sigma}^{\prime}$ est la projection de $\mathbf{u}_{\sigma}$ sur $t_{\mathbf{A}_{\sigma}}(\mathbb{C})$ (le vecteur $\mathbf{u}_{\sigma}^{\prime}$ est aussi un logarithme du point $\mathbf{q}$, défini p. 63). Alors

$$
\begin{aligned}
\left\|\widetilde{\varphi}_{i}(s)(\mathcal{D})\right\|_{\varepsilon_{m \mathbf{p}}^{*} \mathscr{M}, \sigma}= & \left|\sum_{|\underline{i}|=\ell} a_{(0, \underline{i})} \cdot \frac{\left(\frac{\partial}{\partial z_{1}}\right)^{i_{1}} \cdots\left(\frac{\partial}{\partial z_{d}}\right)^{i_{d}}}{i_{1} ! \cdots i_{d} !} F_{s}\left(m \mathbf{u}_{\sigma}+z_{1} b_{1}+\cdots+z_{d} b_{d}\right)(0)\right| \\
& \times \frac{1}{\left(1+\left|m \sigma\left(u_{0}\right)\right|^{2}\right)^{D_{0} / 2}} \times e^{-\frac{\pi}{2} D_{1} m^{2}\left\|\mathbf{u}_{\sigma}^{\prime}\right\|_{L, \sigma}^{2}} \times \delta_{\ell}\left(D_{0}\right) .
\end{aligned}
$$

Cela ne dépend que de la classe de $\mathbf{u}_{\sigma}$ dans $t_{\mathbf{G}_{\sigma}}(\mathbb{C}) / \Omega_{\mathbf{G}_{\sigma}}(\mathbb{C})$ (i.e. seulement de $\sigma(\mathbf{p})$ ). Par définition, on a

$$
\left\|\widetilde{\varphi}_{i}(s)\right\|_{S^{\ell} t_{\mathbf{G}}(\mathbb{C})^{v} \otimes \varepsilon_{m \mathbf{p}}^{*} \mathscr{M}, \sigma}=\sup _{\mathcal{D} \in S^{\ell} t_{\mathbf{G}_{\sigma}}(\mathbb{C}) \backslash\{0\}}\left\{\frac{\left\|\widetilde{\varphi}_{i}(s)(\mathcal{D})\right\|_{\varepsilon_{m \mathbf{p}}^{*} \mathscr{M}, \sigma}}{\|\mathcal{D}\|_{S^{\ell} t_{\mathbf{G}_{\sigma}}(\mathbb{C})}}\right\}
$$

et

$$
\left\|\widetilde{\varphi}_{i}\right\|_{\sigma}=\sup _{s \in \mathscr{E}_{i, \sigma}}\left\{\frac{\left\|\widetilde{\varphi}_{i}(s)\right\|_{S^{\ell} t_{\mathbf{G}}(\mathbb{C})^{v} \otimes \varepsilon_{m \mathbf{p}}^{*} \mathscr{M}, \sigma}}{\|s\|_{\overline{\mathscr{E}}, \sigma}}\right\}
$$

Après cette phase préparatoire, nous pouvons énoncer la

Proposition II.5.7. Pour tout plongement $\sigma: K \hookrightarrow \mathbb{C}$ et tout $i=(\ell, m)$ avec $|\ell| \leq 2(d+1) T$ et $0 \leq m \leq(d+1) S$, on $a$

$$
\left\|\widetilde{\varphi}_{i}\right\|_{\sigma} \leq \frac{\left(4 d D_{0}\right)^{5(d+1) T}}{\mathrm{~d}_{\sigma}\left(e_{0}, W_{\sigma}\right)^{D_{0}}} \times e^{\frac{\pi}{2} d \frac{D_{1}}{D_{0}}\left(\frac{d}{D_{0}}+8 d^{1.5} S\right)} \times \sup _{s \in \mathscr{E}_{i, \sigma} \backslash\{0\}}\left\{\frac{\|s\|_{\infty, \sigma}}{\|s\|_{\mathscr{E}, \sigma}}\right\}
$$

Démonstration. Quitte à réindexer $\left\{e_{1}, \ldots, e_{d}\right\}$, nous pouvons supposer que $e_{1} \notin W$. Considérons $\left(e_{0}, f_{1}, \ldots, f_{d}\right)$ une base orthonormée de $t_{\mathscr{X}_{\sigma}}(\mathbb{C})$ telle que

1. $\left(f_{2}, \ldots, f_{d}\right)$ est l'orthonormalisée de Schmidt de la famille $\left\{\sigma\left(\beta_{j}\right) e_{1}-\sigma\left(\beta_{1}\right) e_{j}\right\}_{2 \leq j \leq d}$ de $W_{\sigma} \cap t_{\mathbf{A}_{\sigma}}(\mathbb{C})$.

2. $f_{1} \in t_{\mathbf{A}_{\sigma}}(\mathbb{C})$.

3. $e_{0}$ est la base canonique de $t_{\mathbb{G}_{a}}$. 
Soit $x_{1}$ la coordonnée relative à $e_{1}$ de $f_{1}$ dans la base $\left(e_{1}, f_{2}, \ldots, f_{d}\right)$ de $t_{\mathbf{A}_{\sigma}}(\mathbb{C})$. On a $x_{1} \neq 0$ et

$$
\begin{aligned}
\mathrm{d}_{\sigma}\left(e_{1}, W_{\sigma} \cap t_{\mathbf{A}_{\sigma}}(\mathbb{C})\right) & =\frac{1}{\left|x_{1}\right|} \mathrm{d}_{\sigma}\left(x_{1} e_{1}, W_{\sigma} \cap t_{\mathbf{A}_{\sigma}}(\mathbb{C})\right) \\
& =\frac{1}{\left|x_{1}\right|} \mathrm{d}_{\sigma}\left(f_{1}, W_{\sigma} \cap t_{\mathbf{A}_{\sigma}}(\mathbb{C})\right) \\
& =\frac{1}{\left|x_{1}\right|} .
\end{aligned}
$$

Comme

$$
\mathrm{d}_{\sigma}\left(e_{1}, W_{\sigma} \cap t_{\mathbf{A}_{\sigma}}(\mathbb{C})\right) \geq \mathrm{d}_{\sigma}\left(e_{1}, W_{\sigma}\right)=\left|\sigma\left(\beta_{1}\right)\right| \mathrm{d}_{\sigma}\left(e_{0}, W_{\sigma}\right)
$$

(car $\left.e_{1}+\sigma\left(\beta_{1}\right) e_{0} \in W_{\sigma}\right)$, on en déduit

$$
\frac{1}{\mathrm{~d}_{\sigma}\left(e_{0}, W_{\sigma}\right)} \geq\left|x_{1} \sigma\left(\beta_{1}\right)\right|
$$

De plus, pour $\underline{i} \in \mathbb{N}^{d+1}$ de longueur $\ell$,

$$
\mathcal{P}_{\ell}\left(e_{0}^{i_{0}} f_{1}^{i_{1}} \cdots f_{d}^{i_{d}}\right)= \begin{cases}\left(f_{1}+x_{1} \sigma\left(\beta_{1}\right) e_{0}\right)^{i_{1}} f_{2}^{i_{2}} \cdots f_{d}^{i_{d}} & \text { si } i_{0}=0 \\ 0 & \text { si } i_{0} \neq 0\end{cases}
$$

D'après l'équation (40), pour $\mathcal{D}=\sum a_{\underline{i}} \cdot e_{0}^{i_{0}} f_{1}^{i_{1}} \cdots f_{d}^{i_{d}} \in S^{\ell} t_{\mathscr{X}_{\sigma}}(\mathbb{C})$ et $s \in \mathscr{E}_{i, \sigma}$, la norme

$$
\left\|\widetilde{\varphi}_{i}(s)(\mathcal{D})\right\|_{\varepsilon_{m \mathbf{p}}^{*} \mathscr{M}, \sigma}
$$

vaut

$$
\begin{aligned}
& \delta_{\ell}\left(D_{0}\right) \times\left\|s_{0}\right\|_{\varepsilon_{m \mathbf{p}}^{*} \mathscr{M}, \sigma} \times \\
& \left|\sum_{|\underline{i}|=\ell} a_{(0, \underline{i})} \frac{\left(\frac{\partial}{\partial z_{1}}+\sigma\left(\beta_{1}\right) x_{1} \frac{\partial}{\partial z_{0}}\right)^{i_{1}}\left(\frac{\partial}{\partial z_{2}}\right)^{i_{2}} \cdots\left(\frac{\partial}{\partial z_{d}}\right)^{i_{d}}}{i_{1} ! \cdots i_{d} !} F_{s}\left(m \mathbf{u}_{\sigma}+z_{0} e_{0}+z_{1} f_{1}+\cdots+z_{d} f_{d}\right)(0)\right| .
\end{aligned}
$$

Comme $F_{s}\left(m \mathbf{u}_{\sigma}+z_{0} e_{0}+z_{1} f_{1}+\cdots+z_{d} f_{d}\right)$ est polynomiale en $z_{0}$ de degré $\leq D_{0}$, on a

$$
\begin{aligned}
& \frac{1}{i_{1} ! \cdots i_{d} !}\left(\frac{\partial}{\partial z_{1}}+\sigma\left(\beta_{1}\right) x_{1} \frac{\partial}{\partial z_{0}}\right)^{i_{1}}\left(\frac{\partial}{\partial z_{2}}\right)^{i_{2}} \cdots\left(\frac{\partial}{\partial z_{d}}\right)^{i_{d}} F_{s}\left(m \mathbf{u}_{\sigma}+z_{0} e_{0}+z_{1} f_{1}+\cdots+z_{d} f_{d}\right) \\
& =\sum_{j_{0}=0}^{\min \left\{i_{1}, D_{0}\right\}}\left(\sigma\left(\beta_{1}\right) x_{1}\right)^{j_{0}} \frac{\left(\frac{\partial}{\partial z_{0}}\right)^{j_{0}}\left(\frac{\partial}{\partial z_{1}}\right)^{i_{1}-j_{0}}\left(\frac{\partial}{\partial z_{2}}\right)^{i_{2}} \cdots\left(\frac{\partial}{\partial z_{d}}\right)^{i_{d}}}{j_{0} !\left(i_{1}-j_{0}\right) ! i_{2} ! \cdots i_{d} !} F_{s}\left(m \mathbf{u}_{\sigma}+z_{0} e_{0}+z_{1} f_{1}+\cdots+z_{d} f_{d}\right) .
\end{aligned}
$$

Donc

$$
\begin{aligned}
\left\|\widetilde{\varphi}_{i}(s)(\mathcal{D})\right\|_{\varepsilon_{m \mathbf{p}}^{*} \mathscr{M}, \sigma} \leq & \delta_{\ell}\left(D_{0}\right) \times \max \left\{1,\left|x_{1} \sigma\left(\beta_{1}\right)\right|\right\}^{D_{0}} \times\left(D_{0}+1\right) \times\left(\sum_{|\underline{i}|=\ell}\left|a_{(0, \underline{i})}\right|\right) \\
& \times \max _{\substack{j \in \mathbb{N} d+1 \\
|\underline{j}|=\ell}}\left\{\left|\frac{1}{j !}\left(\frac{\partial}{\partial \underline{z}}\right)^{\underline{j}} F_{s}\left(m \mathbf{u}_{\sigma}\right)\right|\right\} \times\left\|s_{0}\right\|_{\varepsilon_{m \mathbf{p}}^{*} \mathscr{M}, \sigma} .
\end{aligned}
$$


Or

$$
\sum_{|\underline{i}|=\ell}\left|a_{(0, \underline{i})}\right|=\sum_{|\underline{i}|=\ell}\left|a_{(0, \underline{i})}\right| \sqrt{\frac{\underline{i} !}{\ell !}} \sqrt{\frac{\ell !}{\underline{i} !}} \leq\|\mathcal{D}\|_{S^{\ell} t_{\mathscr{X}_{\sigma}}(\mathbb{C})} \times d^{\ell / 2}
$$

(Cauchy-Schwarz) et, pour tout réel $r>0$,

$$
\left|\frac{1}{\bar{i} !}\left(\frac{\partial}{\partial \underline{z}}\right)^{\underline{i}} F_{s}\left(m \mathbf{u}_{\sigma}\right)\right| \leq \frac{1}{r^{\ell}} \times \max _{\theta_{j} \in[0,2 \pi]}\left\{\left|F_{s}\left(m \mathbf{u}_{\sigma}+r e^{i \theta_{0}} e_{0}+r e^{i \theta_{1}} f_{1}+\cdots+r e^{i \theta_{d}} f_{d}\right)\right|\right\}
$$

donc

$$
\begin{aligned}
\left\|\widetilde{\varphi}_{i}(s)\right\|_{S^{\ell} t_{\mathscr{X}}^{v} \otimes \varepsilon_{m \mathbf{p}}^{*} \mathscr{M}, \sigma} \leq & \frac{\delta_{\ell}\left(D_{0}\right)}{\mathrm{d}_{\sigma}\left(e_{0}, W_{\sigma}\right)^{D_{0}}} \times\left(D_{0}+1\right) \times d^{\ell / 2} \times \sup _{x \in \mathscr{X}_{\sigma}(\mathbb{C})}\left\{\|s(x)\|_{\overline{\mathscr{M}}, \sigma}\right\} \times \frac{1}{r^{\ell}} \\
& \times\left(\frac{1+\left(m\left|\sigma\left(u_{0}\right)\right|+r\right)^{2}}{1+\left(m\left|\sigma\left(u_{0}\right)\right|\right)^{2}}\right)^{D_{0} / 2} \times e^{\frac{\pi}{2} D_{1}\left(\left\|m \mathbf{u}_{\sigma}^{\prime}\right\|_{L, \sigma}+r d\right)^{2}-\frac{\pi}{2} D_{1} m^{2}\left\|\mathbf{u}_{\sigma}^{\prime}\right\|_{L, \sigma}^{2}}
\end{aligned}
$$

(voir l'équation (38) reliant $s$ et $F_{s}$ ). Choisissons $r=\frac{1}{D_{0}}$. En majorant

$$
\frac{1+\left(m\left|\sigma\left(u_{0}\right)\right|+r\right)^{2}}{1+\left(m\left|\sigma\left(u_{0}\right)\right|\right)^{2}}
$$

par $1+2 r$ et $\delta_{\ell}\left(D_{0}\right)$ par $\left(4 D_{0}\right)^{\ell}$ (lemme I.14.2, p. 37$)$, on trouve :

$$
\left\|\widetilde{\varphi}_{i}\right\|_{\sigma} \leq \frac{\left(4 D_{0}\right)^{\ell}}{\mathrm{d}_{\sigma}\left(e_{0}, W_{\sigma}\right)^{D_{0}}} \times d^{(d+1) T} \times e^{\frac{\pi}{2} d \frac{D_{1}}{D_{0}}\left(\frac{d}{D_{0}}+8 d^{1.5} S\right)} \times \sup _{s \in \mathscr{E}_{i, \sigma} \backslash\{0\}}\left\{\frac{\|s\|_{\infty, \sigma}}{\|s\|_{\overline{\mathscr{E}}, \sigma}}\right\} .
$$

Ce qui achève la preuve de la proposition.

\section{Estimation fine des $\left\|\widetilde{\varphi}_{i}\right\|_{\sigma_{0}}$}

Les notations sont les mêmes que celles du paragraphe précédent, mais tous les calculs sont relatifs au plongement initial $\sigma_{0}$ de $K$ dans $\mathbb{C}$ (c'est pourquoi nous omettrons parfois l'indice $\left.\sigma_{0}\right)$. En particulier, $b_{2}, \ldots, b_{d}$ est une base orthonormée de $W \cap t_{\mathbf{A}}(\mathbb{C})$, obtenue par le procédé d'orthonormalisation de Schmidt à partir de $\left\{\beta_{j} e_{1}-\beta_{1} e_{j}\right\}_{j}$. Notons $\tilde{f}_{1}=f_{1}+x_{1} \beta_{1} e_{0}$. La famille $\mathbf{f}=\left\{\tilde{f}_{1}, f_{2}, \ldots, f_{d}\right\}$ est une base de $W \otimes \mathbb{C}$. Nous allons montrer que, pour $\underline{i} \in \mathbb{N}^{d}$ de longueur $\ell$, les (modules des) nombres complexes $\frac{1}{i !} \mathcal{D}_{\underline{\mathbf{f}}}^{\underline{i}} F_{s}(m \mathbf{u})$ sont plus « petits » que le majorant fourni par l'inégalité de Cauchy (comme au $\S$ précédent). La méthode consiste comme dans le cas « classique » (voir $\S$ I.13 de la première partie) — à utiliser la petitesse (en fait ici la nullité) des dérivées (le long de $W$ ) en les points $m^{\prime} \mathbf{u}$ avec $m^{\prime}<m$ et réaliser une extrapolation « à la Baker ».

Rappelons que $\left(u_{0}, u_{1}, \ldots, u_{d}\right)$ désigne les coordonnées de $\mathbf{u}$ dans la base $\left(e_{0}, e_{1}, \ldots, e_{d}\right)$ de $t_{\mathbf{G}}(\mathbb{C})(\operatorname{voir} \S$ II.2). Définissons

$$
\begin{aligned}
\mathbf{w}: & =\left(\beta_{1} u_{1}+\cdots+\beta_{d} u_{d}\right) e_{0}+u_{1} e_{1}+\cdots+u_{d} e_{d} \\
& =u_{1}\left(e_{1}+\beta_{1} e_{0}\right)+\cdots+u_{d}\left(e_{d}+\beta_{d} e_{0}\right) .
\end{aligned}
$$

C'est un vecteur de l'hyperplan dont la distance à $\mathbf{u}$ est exactement $|\Lambda|=\left|u_{0}-\beta_{1} u_{1}-\cdots-\beta_{d} u_{d}\right|$. Avant de démontrer la majoration principale de ce paragraphe, nous aurons besoin du 


\section{Démonstration du théorème}

Lemme II.5.8. Pour tous $s \in \mathscr{E} \mathbb{C}, \underline{t} \in \mathbb{N}^{d}$ et $m^{\prime} \in \mathbb{N}$, on a

$$
\begin{aligned}
\left|\frac{1}{\underline{t} !} \mathcal{D}_{\underline{\mathbf{f}}}^{\underline{t}} F_{s}\left(m^{\prime} \mathbf{u}\right)-\frac{1}{\underline{t} !} \mathcal{D}_{\mathbf{f}}^{\underline{t}} F_{s}\left(m^{\prime} \mathbf{w}\right)\right| \leq & m^{\prime} \times|\Lambda| \times\|s\|_{\infty} \times d^{\mid \underline{\underline{t} \mid+1}} \times\left(\frac{5}{\mathrm{~d}\left(e_{0}, W\right)}\right)^{D_{0}} \\
& \times \max \left\{1, m^{\prime}\left|u_{0}\right|\right\}^{D_{0}} \times e^{\pi D_{1}\left(m^{\prime 2}\left\|\mathbf{u}^{\prime}\right\|_{L, \sigma_{0}}^{2}+1\right)} .
\end{aligned}
$$

Démonstration. D'après l'égalité des accroissements finis, il existe $x_{0} \in[0,1]$ tel que, pour tout $r>0$, on ait

$$
\begin{aligned}
\left|\underline{1} \underline{\underline{t}} \mathcal{D}_{\overline{\mathbf{f}}}^{\underline{t}} F_{s}\left(m^{\prime} \mathbf{u}\right)-\frac{1}{\underline{t} !} \mathcal{D}_{\overline{\mathbf{f}}}^{\underline{t}} F_{s}\left(m^{\prime} \mathbf{w}\right)\right| \\
=m^{\prime}|\Lambda|\left|\frac{\partial}{\partial z_{0}}\left(\frac{1}{\underline{\underline{t}}} \mathcal{D}_{\mathbf{f}}^{\underline{t}} F_{s}\right)\left(m^{\prime} \mathbf{u}-x_{0} m^{\prime} \Lambda . e_{0}\right)\right| \\
\leq m^{\prime} \times|\Lambda| \times \frac{1}{r \mid \underline{\mid \underline{+1}}} \times \max _{\theta_{j} \in[0,2 \pi]}\left|F_{s}\left(m^{\prime} \mathbf{u}-x_{0} m^{\prime} \Lambda . e_{0}+r e^{i \theta_{0}} e_{0}+r e^{i \theta_{1}} \widetilde{f}_{1}+r e^{i \theta_{2}} f_{2}+\cdots+r e^{i \theta_{d}} f_{d}\right)\right| \\
\leq m^{\prime} \times|\Lambda| \times \frac{1}{r \underline{|t|+1}} \times\|s\|_{\infty} \times\left(1+\left(m^{\prime}\left|u_{0}\right|+m^{\prime}|\Lambda|+r\left(1+\left|x_{1} \beta_{1}\right|\right)\right)^{2}\right)^{D_{0} / 2} \\
\quad \times e^{\frac{\pi}{2} D_{1}\left\|m^{\prime} \mathbf{u}^{\prime}+r e^{i \theta_{1}} f_{1}+\cdots+r e^{i \theta_{d}} f_{d}\right\|_{L, \sigma_{0}}^{2}}
\end{aligned}
$$

Nous pouvons supposer que $|\Lambda| \leq\left|u_{0}\right|$, car, dans le cas contraire, le théorème II.2.1 est banal. Choisissons également $r=1 / d$. Alors

$$
\begin{aligned}
\left|\frac{1}{\underline{t} !} \mathcal{D}_{\underline{\mathbf{f}}}^{\underline{t}} F_{s}\left(m^{\prime} \mathbf{u}\right)-\frac{1}{\underline{t} !} \mathcal{D}_{\mathbf{f}}^{\underline{t}} F_{s}\left(m^{\prime} \mathbf{w}\right)\right| \leq & m^{\prime} \times|\Lambda| \times\|s\|_{\infty} \times d^{|\underline{t}|+1} \times\left(\frac{5}{\mathrm{~d}\left(e_{0}, W\right)}\right)^{D_{0}} \\
& \times \max \left\{1, m^{\prime}\left|u_{0}\right|\right\}^{D_{0}} \times e^{\pi D_{1}\left(m^{\prime 2}\left\|\mathbf{u}^{\prime}\right\|_{L, \sigma_{0}}^{2}+1 / d\right)},
\end{aligned}
$$

ce qui conclut la démonstration.

Soit $f$ la fonction analytique d'une variable complexe définie par

$$
f(z)=\frac{1}{\underline{i} !} \mathcal{D}_{\overline{\mathbf{f}}}^{\underline{i}} F_{s}(z \mathbf{w}) \quad \text { où }|\underline{i}| \leq(d+1) T .
$$

Écrivons w dans la base $\left(\widetilde{f}_{1}, f_{2}, \ldots, f_{d}\right)$ de $W$ :

$$
\mathbf{w}=w_{1} \tilde{f}_{1}+w_{2} f_{2}+\cdots+w_{d} f_{d} .
$$

D'après la formule de dérivation à plusieurs variables, pour tout entier naturel $\ell$, on a

$$
\frac{1}{\ell !} f^{(\ell)}(z)=\sum_{|\underline{j}|=\ell}\left(\begin{array}{c}
\underline{i}+\underline{j} \\
\underline{i}
\end{array}\right) w_{1}^{i_{1}} \cdots w_{d}^{i_{d}} \frac{\mathcal{D}_{\mathbf{f}}^{\underline{i}+\underline{j}}}{(\underline{i}+\underline{j}) !} F_{s}(z \mathbf{w})
$$

dont nous déduisons la majoration :

$$
\max _{\substack{0 \leq h \leq S_{0} \\ 0 \leq \ell \leq(d+1) T}}\left\{\frac{1}{\ell !}\left|f^{(\ell)}(h)\right|\right\} \leq\left(d+\left|w_{1}\right|+\cdots+\left|w_{d}\right|\right)^{(d+1) T} \times \max _{\substack{0 \leq h \leq S_{0} \\|\underline{j}| \leq 2(d+1) T}}\left\{\frac{1}{j !}\left|\mathcal{D}_{\mathbf{f}}^{j} F_{s}(h \mathbf{w})\right|\right\} .
$$

Or, d'une part, le réel $\left|w_{1}\right|+\cdots+\left|w_{d}\right|$ est majoré par $\sqrt{d}\left(\left|w_{1}\right|^{2}+\cdots+\left|w_{d}\right|^{2}\right)^{1 / 2}=\sqrt{d}\left\|\mathbf{u}^{\prime}\right\|_{L, \sigma_{0}}$ où $\mathbf{u}^{\prime}=w_{1} f_{1}+\cdots+w_{d} f_{d}$ est la projection de $\mathbf{u}$ sur $t_{\mathbf{A}}(\mathbb{C})$ et, d'autre part, la nullité du terme 
$\mathcal{D}_{\mathbf{f}}^{j} F_{s}(h \mathbf{u})$ (pour $|\underline{j}| \leq 2(d+1) T$ et $0 \leq h \leq S_{0}$ ) entraîne une majoration de $\left|\frac{1}{\underline{j} !} \mathcal{D}_{\mathbf{f}}^{j} F_{s}(h \mathbf{w})\right|$, en utilisant le lemme II.5.8 précédent. Avec l'inégalité (47), nous obtenons alors

$$
\begin{aligned}
\max _{\substack{0 \leq h \leq S_{0} \\
0 \leq \ell \leq(d+1) T}}\left\{\frac{1}{\ell !}\left|f^{(\ell)}(h)\right|\right\} \leq & d^{3(d+1) T}\left(1+\left\|\mathbf{u}^{\prime}\right\|_{L, \sigma_{0}}\right)^{(d+1) T} S_{0}\left(\frac{14}{\mathrm{~d}\left(e_{0}, W\right)}\right)^{D_{0}} \\
& \times \max \left\{1, S_{0}\left|u_{0}\right|\right\}^{D_{0}} e^{D_{1}\left(\pi S_{0}^{2}\left\|\mathbf{u}^{\prime}\right\|_{L, \sigma_{0}}^{2}+\pi\right)}|\Lambda| \times\|s\|_{\infty} .
\end{aligned}
$$

Lemme II.5.9. Soit $E$ un réel $\geq 1$. Alors

$$
|f|_{4 m E} \leq d^{(d+1) T}\left(\frac{8 m E}{\mathrm{~d}\left(e_{0}, W\right)}\right)^{D_{0}} e^{\pi D_{1}\left(16 m^{2} E^{2}\left\|\mathbf{u}^{\prime}\right\|_{L, \sigma_{0}}^{2}+1\right)}\|s\|_{\infty}
$$

Démonstration. Posons $R:=4 m E$. D'après l'inégalité de Cauchy, pour $|z| \leq R$, on a

$$
\begin{aligned}
|f(z)| & \leq \frac{1}{r|\underline{i}|} \times \sup _{\theta_{i}}\left\{\left|F_{s}\left(z \mathbf{w}+r e^{i \theta_{1}} \widetilde{f}_{1}+\cdots+r e^{i \theta_{d}} f_{d}\right)\right|\right\} \\
& \leq \frac{1}{r|\underline{i}|} \times\left(1+(R+r)^{2}\left|x_{1} \beta_{1}\right|^{2}\right)^{D_{0} / 2} \times e^{\frac{\pi}{2} D_{1}\left(\left(R\left|w_{1}\right|+r\right)^{2}+\cdots+\left(R\left|w_{d}\right|+r\right)^{2}\right)} \times\|s\|_{\infty}
\end{aligned}
$$

donc, en choisissant $r=1 / d$ et comme $R=4 m E$ (où $E \geq 1$ ), nous obtenons

$$
|f|_{R} \leq d^{|\underline{\mid}|} \times\left(\frac{8 m E}{\mathrm{~d}\left(e_{0}, W\right)}\right)^{D_{0}} \times e^{\pi D_{1}\left(16 m^{2} E^{2}\left\|\mathbf{u}^{\prime}\right\|_{L, \sigma_{0}}^{2}+1 / d\right)} \times\|s\|_{\infty}
$$

puis le lemme.

Proposition II.5.10. Pour tout $i \geq 2\left(S_{0}+1\right)(d+1) T+1$, on a

$$
\begin{aligned}
\left\|\widetilde{\varphi}_{i}\right\|_{\sigma_{0}} \leq & S(d+1)^{4(d+1) T} e^{\pi D_{1}}\left(\frac{60 m}{\mathrm{~d}\left(e_{0}, W\right)}\right)^{D_{0}} \times \sup _{s \in \mathscr{E}_{i, \sigma_{0}}}\left\{\frac{\|s\|_{\infty, \sigma_{0}}}{\|s\|_{\overline{\mathscr{E}}, \sigma_{0}}}\right\} \\
& \times\left\{\left(\frac{18(d+1) S}{S_{0}}\right)^{(d+1) T S_{0}}\left(1+\left\|\mathbf{u}^{\prime}\right\|_{L, \sigma_{0}}\right)^{(d+1) T}|\Lambda|+\left(\frac{1}{E}\right)^{(d+1) T S_{0}-D_{0}} e^{\left.200 d^{2} D_{1} S^{2} E^{2}\left\|\mathbf{u}^{\prime}\right\|_{L, \sigma_{0}}^{2}\right\}}\right.
\end{aligned}
$$

Démonstration. Utilisons la formule d'extrapolation du lemme I.13.3 (page 35) avec $R=4 m E$, $r=m, T_{1}:=(d+1) T, S_{1}:=S_{0}$. Avec les inégalités (48) et (49), et comme $m \leq(d+1) S$, nous obtenons :

$$
\begin{aligned}
|f|_{(d+1) S} \leq & 2\left(\frac{1}{E}\right)^{(d+1) T S_{0}} \times|f|_{4 m E}+5\left(\frac{18(d+1) S}{S_{0}}\right)^{(d+1) T S_{0}} \times \max _{\substack{0 \leq h \leq S_{0} \\
0 \leq \ell \leq(d+1) T}}\left\{\frac{1}{\ell !}\left|f^{(\ell)}(h)\right|\right\} \\
\leq & \max \left\{1,\left|m u_{0}\right|\right\}^{D_{0}} d^{3(d+1) T} S_{0}\left(\frac{14 m}{\mathrm{~d}\left(e_{0}, W\right)}\right)^{D_{0}} e^{\pi D_{1}\left(1+S_{0}^{2}\left\|\mathbf{u}^{\prime}\right\|_{L, \sigma_{0}}\right)}\|s\|_{\infty, \sigma_{0}} \\
& \times\left\{\left(\frac{18(d+1) S}{S_{0}}\right)^{(d+1) T S_{0}}\left(1+\left\|\mathbf{u}^{\prime}\right\|_{L, \sigma_{0}}\right)^{(d+1) T}|\Lambda|\right. \\
& \left.+\left(\frac{1}{E}\right)^{(d+1) T S_{0}-D_{0}} e^{16 \pi D_{1}(d+1)^{2} S^{2} E^{2}\left\|\mathbf{u}^{\prime}\right\|_{L, \sigma_{0}}^{2}}\right\} .
\end{aligned}
$$


Le lemme II.5.8 fournit alors la majoration

$$
\begin{aligned}
\mid \underline{\frac{1}{i} !} \mathcal{D}_{\overline{\mathbf{f}}}^{\underline{i} F_{s}(m \mathbf{u}) \mid \leq} & S(d+1)^{3(d+1) T}\left(\frac{14 m}{\mathrm{~d}\left(e_{0}, W\right)}\right)^{D_{0}} \max \left\{1,\left|m u_{0}\right|\right\}^{D_{0}} e^{\pi D_{1}\left(m^{2}\left\|\mathbf{u}^{\prime}\right\|_{L, \sigma_{0}}^{2}+1\right)}\|s\|_{\infty, \sigma_{0}} \\
& \times\left\{\left(\frac{18(d+1) S}{S_{0}}\right)^{(d+1) T S_{0}}\left(1+\left\|\mathbf{u}^{\prime}\right\|_{L, \sigma_{0}}\right)^{(d+1) T}|\Lambda|\right. \\
& \left.+\left(\frac{1}{E}\right)^{(d+1) T S_{0}-D_{0}} e^{16 \pi D_{1}(d+1)^{2} S^{2} E^{2}\left\|\mathbf{u}^{\prime}\right\|_{L, \sigma_{0}}^{2}}\right\} .
\end{aligned}
$$

Puis en revenant à $\widetilde{\varphi}_{i}$ via les égalités (41), (42) et (43), nous obtenons la majoration de la proposition.

\section{Hauteur des morphismes}

Proposition II.5.11. Rappelons que $h^{\prime}(W)$ désigne la somme

$$
\frac{1}{[K: \mathbb{Q}]} \sum_{\sigma: K \hookrightarrow \mathbb{C}} \log \frac{1}{\mathrm{~d}_{\sigma}\left(e_{0}, W_{\sigma}\right)} .
$$

Alors

(1) Pour tout entier $i$, on a

$$
\begin{aligned}
h\left(\widetilde{\varphi}_{i}\right) \leq 5 & (d+1) T \log \left(4 d D_{0}\right)+4 \pi d^{2.5} \frac{S D_{1}}{D_{0}}+D_{0} h^{\prime}(W) \\
& +\log \left(D_{0}+1\right)+d \log D_{1}+\frac{d^{2}}{4} \max \left\{1, h_{F}(\mathbf{A})\right\}+4 d^{4}
\end{aligned}
$$

(2) Pour $i \geq 2(d+1) T\left(S_{0}+1\right)+1$, on a

$$
\begin{aligned}
h\left(\widetilde{\varphi}_{i}\right) \leq & 5(d+1) T \log \left(4 d D_{0}\right)+4 \pi d^{2.5} \frac{S D_{1}}{D_{0}}+D_{0}\left(h^{\prime}(W)+5\right)+\log S \\
& +(\pi+d) D_{1}+\frac{d^{2}}{4} \max \left\{1, h_{F}(\mathbf{A})\right\}+4 d^{4} \\
& +\frac{1}{[K: \mathbb{Q}]} \log \left\{\left(\frac{18(d+1) S}{S_{0}}\right)^{(d+1) T S_{0}}\left(1+\left\|\mathbf{u}^{\prime}\right\|_{L, \sigma_{0}}\right)^{(d+1) T}|\Lambda|\right. \\
& \left.+\left(\frac{1}{E}\right)^{(d+1) T S_{0}-D_{0}} e^{200 d^{2} D_{1} S^{2} E^{2}\left\|\mathbf{u}^{\prime}\right\|_{L, \sigma_{0}}^{2}}\right\}
\end{aligned}
$$

Remarque : on notera l'absence dans ces majorants des hauteurs de $u_{0}$ et de $\mathbf{q}$, conséquence du choix des métriques.

La démonstration de cette proposition est immédiate à partir de la définition de la hauteur de $\widetilde{\varphi}_{i}$ et des évaluations des normes archimédiennes de ces morphismes, effectuées précédemment. 


\section{Le cas abélien non-homogène}

\section{$\S 5 . g$. Conclusion}

À partir de maintenant, nous allons raisonner par l'absurde en supposant que

$$
\left(\frac{18(d+1) S}{S_{0}}\right)^{(d+1) T S_{0}}\left(1+\left\|\mathbf{u}^{\prime}\right\|_{L, \sigma_{0}}\right)^{(d+1) T}|\Lambda| \leq\left(\frac{1}{E}\right)^{(d+1) T S_{0}-D_{0}} e^{200 d^{2} D_{1} S^{2} E^{2}\left\|\mathbf{u}^{\prime}\right\|_{L, \sigma_{0}}^{2}} .
$$

Dans ce cas, pour $i \geq 2(d+1) T\left(S_{0}+1\right)+1$, on a

$$
\begin{aligned}
h\left(\widetilde{\varphi}_{i}\right) \leq & 5(d+1) T \log \left(4 d D_{0}\right)+\log S+D_{0}\left(h^{\prime}(W)+5+\frac{\log ((d+1) S)}{[K: \mathbb{Q}]}+\frac{\log E}{[K: \mathbb{Q}]}\right) \\
& +D_{1}\left(4 \pi d^{2.5} \frac{S}{D_{0}}+\pi+d+200 d^{2} \frac{S^{2} E^{2}\left\|\mathbf{u}^{\prime}\right\|_{L, \sigma_{0}}^{2}}{[K: \mathbb{Q}]}\right)+\frac{d^{2}}{4} \max \left\{1, h_{F}(\mathbf{A})\right\}+4 d^{4} \\
& -\frac{(d+1) T S_{0}}{[K: \mathbb{Q}]} \log E .
\end{aligned}
$$

Le morphisme $\varphi$ est injectif d'après la proposition II.5.1. Appliquons alors l'inégalité de pentes (12) (page 74).

$$
\widehat{\operatorname{deg}}_{\mathrm{n}} \overline{\mathscr{E}} \leq\left(\operatorname{rg} \mathscr{E}-\operatorname{rg} \mathscr{E}_{i_{0}}\right) \max _{i \leq i_{0}-1}\left\{h\left(\widetilde{\varphi}_{i}\right)+\widehat{\mu}_{\max }\left(\overline{\widetilde{G}_{i}}\right)\right\}+\operatorname{rg} \mathscr{E}_{i_{0}} \max _{i_{0} \leq i \leq \mathcal{N}}\left\{h\left(\widetilde{\varphi}_{i}\right)+\widehat{\mu}_{\max }\left(\overline{\widetilde{G}}_{i}\right)\right\}
$$

(l'entier $i_{0}$ est défini dans le lemme II.5.6, page 85). Grâce à la proposition II.5.3, si $D_{1} \geq 3$ alors

$$
\frac{\widehat{\operatorname{deg}}_{\mathrm{n}} \overline{\mathscr{E}}}{\operatorname{rg} \mathscr{E}} \geq-\frac{h_{F}(\mathbf{A})}{2} .
$$

Ces inégalités entraînent la positivité de

$$
\begin{aligned}
& d^{2} \max \left\{1, h_{F}(\mathbf{A})\right\}+5(d+1) T\left(\log \left(4 d D_{0}\right)+(d+1) h_{F}(\mathbf{A})+6 d^{5}\right) \\
& +D_{0}\left(h\left(u_{0}\right)+h^{\prime}(W)+5+3 \log (d S)+\frac{\log E}{[K: \mathbb{Q}]}\right) \\
& +D_{1}\left(4 \pi d^{2.5} \frac{S}{D_{0}}+\pi+d+200 d^{2} \frac{S^{2} E^{2}\left\|\mathbf{u}^{\prime}\right\|_{L, \sigma_{0}}^{2}}{[K: \mathbb{Q}]}+3(d+1)^{2} S^{2} \widehat{h}(\mathbf{q})\right) \\
& -\frac{(d+1) T S_{0}}{2[K: \mathbb{Q}]} \log E .
\end{aligned}
$$

Après un récapitulatif des principales contraintes et du résultat que nous venons d'obtenir, nous effectuerons un choix de paramètres dont nous déduirons le théorème II.2.1.

\section{Résultat intermédiaire}

Faisons le point sur ces calculs : supposons

$$
\begin{aligned}
& \text { - } x_{0} \geq \frac{S+1}{C_{0}(\operatorname{deg} \mathbf{G}) \widetilde{D}_{0}} \times\left(\frac{\widetilde{T}}{\widetilde{D}_{1}}\right)^{d} \\
& \text { - } S+1 \geq(3 / 2)^{d} C_{0} \\
& \text { - } x_{0} \widetilde{D}_{1} \geq 3 \\
& \text { - } C_{0} \geq 4^{d} d !+1
\end{aligned}
$$


- $\widetilde{T} \geq 6^{d+1}(d !)^{2} \max \left\{\widetilde{D}_{1}, \frac{\widetilde{D}_{0}}{S+1}\right\}$

- $|\Lambda| \leq\left|u_{0}\right|$

- $S / S_{0} \geq 8^{d+1}(d+1)^{d+1} C_{0}$

$-d \geq 1$

- $T S_{0} \log E \geq 40[K: \mathbb{Q}] d \max \left\{1, h_{F}(\mathbf{A})\right\}$

- $S_{0} \log E \geq 40[K: \mathbb{Q}]\left(11 d^{5}+(d+1) h_{F}(\mathbf{A})+\log D_{0}\right)$

- $T S_{0} \log E \geq 4000 d^{3}[K: \mathbb{Q}] D_{1}\left(1+\frac{S}{D_{0}}+\frac{S^{2} E^{2}\left\|\mathbf{u}^{\prime}\right\|_{L, \sigma_{0}}^{2}}{[K: \mathbb{Q}]}+S^{2} \widehat{h}(\mathbf{q})\right)$

- $T S_{0} \log E \geq 40[K: \mathbb{Q}] D_{0} d\left(h\left(u_{0}\right)+h^{\prime}(W)+\log S+\frac{\log E}{[K: \mathbb{Q}]}\right)$

Alors

$$
|\Lambda| \geq\left(\frac{S_{0}}{18(d+1) S}\right)^{(d+1) T S_{0}} \times\left(1+\left\|\mathbf{u}^{\prime}\right\|_{L, \sigma_{0}}\right)^{-(d+1) T} \times\left(\frac{1}{E}\right)^{(d+1) T S_{0}-D_{0}} .
$$

Sous cette forme, la minoration est un peu indigeste. Nous allons choisir des paramètres qui donnent le théorème II.2.1. Le lecteur qui reprend les calculs se convaincra aisément que ce sont les meilleurs possibles, à la dimension $d$ et à la constante numérique près.

\section{Choix concret des paramètres}

Nous allons donner un choix des différents paramètres qui apparaissent dans les conditions ci-dessus. Les constantes numériques sont très (voire excessivement) larges, mais pour l'invariant « hauteur de Faltings » lié à la variété abélienne, nous avons essayé de conserver le «bon » ordre de grandeur, qui ressort naturellement des conditions.

Rappelons que $D$ est un majorant de $[K: \mathbb{Q}]$; choisissons

$$
C_{0}=4^{d} d !+1, \quad S=(8 d)^{4 d} S_{0}-1
$$

et posons

$$
\log a_{0}:=40 D d^{3}\left(h\left(u_{0}\right)+h^{\prime}(W)+\log S_{0}+\frac{\log E}{D}\right)
$$

et

$$
\log a_{1}:=(10 d)^{10 d} D\left(1+\frac{S_{0}}{D_{0}}+\frac{S_{0}^{2} E^{2}\left\|\mathbf{u}^{\prime}\right\|_{L, \sigma_{0}}^{2}}{D}+S_{0}^{2} \widehat{h}(\mathbf{q})\right) .
$$

Choisissons également

$$
x_{0}:=\frac{(8 d)^{4 d} S_{0}}{\widetilde{D}_{0}}\left(\frac{\widetilde{T}}{\widetilde{D}_{1}}\right)^{d} .
$$

Considérons $U_{0}$ un réel $>0$ et posons

$$
\widetilde{T}:=\frac{U_{0}}{S_{0} \log E} \quad, \quad \widetilde{D}_{0}:=\frac{U_{0}}{\log a_{0}} \quad \text { et } \quad \widetilde{D}_{1}:=\frac{U_{0}}{\log a_{1}+(10 d)^{10 d} S_{0} \log E} .
$$

Ces choix de $\widetilde{T}, \widetilde{D}_{0}, \widetilde{D}_{1}$ en fonction de $U_{0}$ impliquent que

$$
x_{0} U_{0}=(8 d)^{4 d} S_{0}\left(\log a_{0}\right)\left((10 d)^{10 d}+\frac{\log a_{1}}{S_{0} \log E}\right)^{d}
$$




\section{Le cas abélien non-homogène}

et, par conséquent (en revenant aux définitions (59)),

$$
x_{0} \widetilde{D}_{0}=(8 d)^{4 d} S_{0}\left((10 d)^{10 d}+\frac{\log a_{1}}{S_{0} \log E}\right)^{d} \quad \text { et } \quad x_{0} \widetilde{D}_{1}=(8 d)^{4 d} \frac{\log a_{0}}{\log E}\left((10 d)^{10 d}+\frac{\log a_{1}}{S_{0} \log E}\right)^{d-1} \text {. }
$$

Il ressort de ces choix que si, de plus,

$$
S_{0} \log E \geq(10 d)^{10} D\left(\max \left\{1, h_{F}(\mathbf{A})\right\}+\log \left(x_{0} \widetilde{D}_{0}\right)\right)
$$

(cette condition étant facile à réaliser dès que $S_{0}$ est très grand) alors nous obtenons la minoration de $|\Lambda|$ donnée par (57). Cette minoration s'écrit simplement

$$
\log |\Lambda| \geq-(100 d)^{100 d} x_{0} U_{0} .
$$

Pour déduire le théorème II.2.1 à partir de cette inégalité, nous choisissons l'entier $S_{0}$ égal à la partie entière de

$$
(20 d)^{20} \frac{D}{\log E}\left(\max \left\{1, h_{F}(\mathbf{A})\right\}+\log ^{+}\left(\frac{D \log a}{\log E}\right)\right)+1
$$

où - nous le rappelons - $\log a$ est un majorant de

$$
\max \left\{1, \widehat{h}(\mathbf{q}), \frac{E^{2}\left\|\mathbf{u}^{\prime}\right\|_{L, \sigma_{0}}^{2}}{D}\right\} .
$$

Par ailleurs, d'après l'égalité (60), on a

$$
x_{0} U_{0} \leq(100 d)^{100 d^{2}} S_{0}^{d+1}\left(\log a_{0}\right)\left(1+\frac{D \log a}{\log E}\right)^{d},
$$

ce qui donne le théorème II.2.1 en remplaçant $S_{0}$ par sa valeur ci-dessus (63) (on notera que le nombre réel

$$
\left(1+\left|\beta_{1}\right|^{2}+\cdots+\left|\beta_{d}\right|^{2}\right)^{1 / 2}
$$

est égal à $\frac{1}{\mathrm{~d}_{\sigma_{0}}\left(e_{0}, W_{\mathbb{C}}\right)}$, et ce nombre est donc inférieur à $b^{D}$, ce qui permet d'obtenir la minoration de $\mathrm{d}_{\sigma_{0}}(\mathbf{u}, W)$ à partir de celle de $\left.|\Lambda|\right)$. 


\section{Annexe B}

\section{Inégalités de pentes et Transcendance (d'après J.-B. BosT)}

Ce texte a été exposé lors d'une séance du groupe de travail de théorie des nombres de Paris 6. Il est essentiellement issu du cours de DEA de J.-B. Bost, donné à l'IHP au second semestre 1997 et de son article Périodes et isogénies des variétés abéliennes sur les corps de nombres. Il a été enrichi des remarques faites ce jour là par les participants à ce groupe de travail, tout en restant, néanmoins, minimaliste...

\section{$\S$ B.1. Définitions}

Soit $K$ un corps de nombres.

Définition B.1.1. Un fibré vectoriel hermitien $\bar{E} \operatorname{sur} \operatorname{Spec} \mathcal{O}_{K}$ est la donnée d'un faisceau localement libre cohérent $E$ sur Spec $\mathcal{O}_{K}$ et d'une structure hermitienne $C^{\infty}$, invariante par conjugaison complexe, sur le fibré vectoriel holomorphe $E_{\mathbb{C}} \operatorname{sur}\left(\operatorname{Spec} \mathcal{O}_{K}\right)(\mathbb{C})$. De facon équivalente, c'est la donnée d'un $\mathcal{O}_{K}$-module $E$ projectif de type fini et d'une famille de normes hermitiennes $\left(\|\cdot\|_{\sigma}\right)_{\sigma: K \hookrightarrow \mathbb{C}}$ sur $E_{\sigma}=E \otimes_{\sigma} \mathbb{C}$ (structure euclidienne si le plongement $\sigma$ est réel, structure hermitienne si $\sigma$ est complexe), compatible avec l'isomorphisme $E_{\sigma} \simeq E_{\bar{\sigma}}$ induit par la conjugaison complexe.

Rappelons que si $\mathfrak{E}$ est un $\mathbb{C}$-espace vectoriel (de dimension finie) muni d'une norme hermitienne $\|$.$\| alors le déterminant de \mathfrak{E}$, noté det $\mathfrak{E}$, est la puissance extérieure maximale de $\mathfrak{E}$. De plus, si on note $\mathfrak{e}$ la dimension de $\mathfrak{E}$, l'espace $\mathfrak{E}$ est naturellement muni de la norme (hermitienne) $\wedge^{\mathfrak{e}}\|\cdot\|$, définie par :

$$
\left(\wedge^{\mathfrak{e}}\|\cdot\|\right)\left(x_{1} \wedge \ldots \wedge x_{\mathfrak{e}}\right)=\operatorname{det}\left(\left(x_{i}, x_{j}\right)\right)_{1 \leq i, j \leq \mathfrak{e}} .
$$

Définition B.1.2. Soient $\bar{E}$ un fibré en droite hermitien sur $\operatorname{Spec} \mathcal{O}_{K}$ et $x$ un élément de $E_{K} \backslash\{0\}=E \otimes_{\mathcal{O}_{K}} K \backslash\{0\}$. Le degré d'Arakelov de $\bar{E}$ est :

$$
\widehat{\operatorname{deg}} \bar{E}:=\sum_{\mathfrak{p}} v_{\mathfrak{p}}(x) \log \mathrm{N}(\mathfrak{p})-\sum_{\sigma} \log \|x\|_{\sigma}
$$

où $\mathrm{N}(\mathfrak{p})=\operatorname{card}\left(\mathcal{O}_{K} / \mathfrak{p}\right), \mathfrak{p}$ parcourant les places finies de $K$ et $\sigma$ les places infinies. Dans le cas général d'un fibré vectoriel hermitien $\bar{E}=(E,\|\|$.$) quelconque, le degré d'Arakelov de \bar{E}$ 
est le degré de $\operatorname{det}(E)$ muni des métriques de type (B.1). Le degré d'Arakelov normalisé (noté $\widehat{\operatorname{deg}_{n}} \bar{E}$ ) est le quotient de $\widehat{\operatorname{deg}} \bar{E} \operatorname{par}[K: \mathbb{Q}]$.

La formule du produit implique que cette définition ne dépend pas du choix de $x$.

Exemple : Un fibré vectoriel hermitien sur Spec $\mathbb{Z}$ est la donnée d'un $\mathbb{Z}$-module libre $\Gamma$ de rang $r$ et d'une norme euclidienne sur $\Gamma \otimes_{\mathbb{Z}} \mathbb{R}$ rendant isométrique $\Gamma \otimes_{\mathbb{Z}} \mathbb{R}$ et $\mathbb{R}^{r}$ muni de sa norme euclidienne. Nous avons alors l'inégalité

$$
\widehat{\operatorname{deg}} \bar{E}=-\log \operatorname{covol}(\Gamma,\|\cdot\|) \text {. }
$$

Il existe une formule plus générale lorsque $\bar{E}$ est un fibré vectoriel hermitien sur Spec $\mathcal{O}_{K}$ :

$$
\widehat{\operatorname{deg}} \bar{E}=-\log V+\frac{\operatorname{rg} E}{2} \log \left|D_{K}\right|
$$

où $V$ est le covolume de $E$, vu comme $\mathbb{Z}$-module, dans $\oplus_{\sigma: K \hookrightarrow \mathbb{C}} E_{\sigma}$ et $\left|D_{K}\right|$ est la valeur absolue du discriminant de $K_{\mid \mathbb{Q}}$. Cette formule est l'analogue de la formule de Riemann-Roch (en géométrie algébrique complexe), où la caractéristique d'Euler de $\bar{E}$ serait $-\log V$.

\section{$\S$ B.2. Pentes des fibrés vectoriels hermitiens}

Soit $\bar{E}$ un fibré vectoriel hermitien $\operatorname{sur} \operatorname{Spec} \mathcal{O}_{K}, V$ un sous $\mathcal{O}_{K}$-module de $E$, que l'on munit de la métrique induite. Ainsi $V$ a un rang et un degré d'Arakelov. On pose $V_{\text {sat }}=V_{K} \cap E$ (sat=saturé). On montre que $V=V_{\text {sat }}$ si et seulement si $V$ est facteur direct dans $E$ (comme $\mathcal{O}_{K}$-module). Par ailleurs

$$
\widehat{\operatorname{deg}}_{\mathrm{n}} \bar{V} \leq \widehat{\operatorname{deg}}_{\mathrm{n}} \bar{V}_{\text {sat }}
$$

En interprétant $\widehat{\operatorname{deg}}_{\mathrm{n}} \bar{V}_{\text {sat }}$ comme l'opposé de la hauteur (dans une Grassmanienne) de $V_{K}$, on montre qu'il n'y a qu'un nombre fini de points du graphe $\left(\operatorname{rg} V, \widehat{\operatorname{deg}}_{\mathrm{n}} \bar{V}_{\text {sat }}\right)$ dans tout demi-plan supérieur. On peut alors considérer l'enveloppe convexe de ces points et obtenir une fonction affine par morceaux, concave $P_{\bar{E}}:[0, \mathrm{rg} E] \rightarrow \mathbb{R}$. On notera $\widehat{\mu}_{i}(\bar{E})$ la pente de $P_{\bar{E}} \operatorname{sur}[i-1, i]$ :

$$
\widehat{\mu}_{i}(\bar{E}):=P_{\bar{E}}(i)-P_{\bar{E}}(i-1)
$$

et

$$
\widehat{\mu}_{\max }(\bar{E}):=\widehat{\mu}_{1}(\bar{E}) \geq \ldots \geq \widehat{\mu}_{\operatorname{rg} E}(\bar{E})=: \widehat{\mu}_{\min }(\bar{E})
$$

(l'inégalité $\widehat{\mu}_{i}(\bar{E}) \geq \widehat{\mu}_{i+1}(\bar{E})$ provient de la concavité de $P_{\bar{E}}$ ). Nous dirons que $\bar{E}$ est semistable si $P_{\bar{E}}(x)$ est fonction linéaire de $x$. Par exemple, le fibré hermitien trivial $\mathcal{O}_{K}^{n}$, muni des métriques hermitiennes relatives à la base canonique, est semi-stable, et même, $P_{\mathcal{O}_{K}^{n}} \equiv 0$.

\section{$\S$ B.3. Notion de pentes pour les morphismes entre fibrés vectoriels hermitiens}

Après avoir explicité la norme d'opérateur que nous choisissons pour une application linéaire $\varphi$ entre espaces vectoriels sur un des complétés ( $\mathfrak{p}$-adique ou archimédien) de $K$ (théorie locale), nous définissons la $i^{\text {ème }}$ pente de $\varphi$ (théorie globale). Ce paragraphe ne contient quasiment aucun résultat; il fixe les notations. 


\section{$\S$ B.3.a. Théorie locale}

- Places finies

On note $\mathcal{O}_{\mathfrak{p}}$ l'anneau des entiers du corps p-adique $K_{\mathfrak{p}}$. Soit $\varphi: E_{K_{\mathfrak{p}}} \rightarrow F_{K_{\mathfrak{p}}}$ une application $K_{\mathfrak{p}}$-linéaire entre $K_{\mathfrak{p}}$-espaces vectoriels. Le corps $K_{\mathfrak{p}}$ est muni de sa norme $\mathfrak{p}$-adique habituelle :

$$
\|\lambda\|_{\mathfrak{p}}:=N(\mathfrak{p})^{-v_{\mathfrak{p}}(\lambda)}, \quad \lambda \in K_{\mathfrak{p}} \backslash\{0\} .
$$

Cette norme induit des normes sur $E_{K_{\mathfrak{p}}}$ et $F_{K_{\mathfrak{p}}}$ : si par exemple $\left(e_{1}, \ldots, e_{h}\right)$ est une base de $E_{K_{\mathfrak{p}}}$ sur $\mathcal{O}_{\mathfrak{p}}$,

$$
\left\|\sum_{i=1}^{h} \lambda_{i} e_{i}\right\|:=\max _{1 \leq i \leq h}\left\|\lambda_{i}\right\|_{\mathfrak{p}} .
$$

Cela ne dépend pas de la base choisie.

On peut considérer alors

$$
\|\varphi\|_{\mathfrak{p}}:=\sup _{\substack{v \in E_{K_{\mathfrak{p}}},\|v\| \leq 1}}\|\varphi(v)\|
$$

On pose également

$$
M_{i, \mathfrak{p}}:=\frac{\left\|\bigwedge^{i} \varphi\right\|_{\mathfrak{p}}}{\left\|\bigwedge^{i-1} \varphi\right\|_{\mathfrak{p}}}
$$

- Places infinies

On se place sur $\mathbb{C}$ (sur $\mathbb{R}$, c'est pareil). Soient $E$ et $F$ deux $\mathbb{C}$-espaces vectoriels de dimension finie munis de structures hermitiennes, et $\varphi: E \rightarrow F$ une application $\mathbb{C}$ linéaire. On pose alors

$$
M_{i}(\varphi):=\min \left\|\varphi_{\mid V}\right\|=\min \max _{\substack{x \in V,\|x\| \leq 1}}\{\|\varphi(x)\|\}
$$

où le minimum porte sur les sous-espaces vectoriels $V$ de $E$ de codimension $i-1$.

\section{$\S$ B.3.b. Théorie globale}

Soient $\bar{E}$ et $\bar{F}$ deux fibrés vectoriels hermitiens sur $\operatorname{Spec} \mathcal{O}_{K}$ et $\varphi_{K}: E_{K} \rightarrow F_{K}$ une application $K$-linéaire. On pose

$$
h\left(\bar{E}, \bar{F}, \varphi_{K}\right):=\frac{1}{[K: \mathbb{Q}]}\left(\sum_{\mathfrak{p}} \log \left\|\varphi_{K}\right\|_{\mathfrak{p}}+\sum_{\sigma: K \hookrightarrow \mathbb{C}} \log \left\|\varphi_{K}\right\|_{\sigma}\right) .
$$

Le réel $h\left(\bar{E}, \bar{F}, \varphi_{K}\right)$ est invariant par extension des scalaires i.e. si $f: \operatorname{Spec} \mathcal{O}_{K^{\prime}} \rightarrow \operatorname{Spec} \mathcal{O}_{K}$ est un morphisme de schémas, on a $h\left(f^{*} \bar{E}, f^{*} \bar{F}, \varphi_{K^{\prime}}\right)=h\left(\bar{E}, \bar{F}, \varphi_{K}\right)$. De plus, pour tout $\lambda \in K \backslash\{0\}$ on a $h\left(\bar{E}, \bar{F}, \lambda \varphi_{K}\right)=h\left(\bar{E}, \bar{F}, \varphi_{K}\right)$ donc $h(\bar{E}, \bar{F},$.$) définit une fonction sur$ $\mathbb{P}(\operatorname{Hom}(E, F))(\bar{K})$ qui est une « hauteur ». On pose également, pour $1 \leq i \leq \operatorname{rg} \varphi$,

$$
\mu_{i}\left(\bar{E}, \bar{F}, \varphi_{K}\right):=\frac{1}{[K: \mathbb{Q}]}\left(\sum_{\mathfrak{p}} \log M_{i, \mathfrak{p}}\left(\varphi_{K}\right)+\sum_{\sigma: K \hookrightarrow \mathbb{C}} \log M_{i}\left(\varphi_{K, \sigma}\right)\right) .
$$

La quantité $\mu_{i}$ est invariante par extension des scalaires. Pour fixer les idées, on a

$$
\mu_{1}\left(\bar{E}, \bar{F}, \varphi_{K}\right)=h\left(\bar{E}, \bar{F}, \varphi_{K}\right)
$$


et

$$
\mu_{i}\left(\bar{E}, \bar{F}, \varphi_{K}\right)=h\left(\wedge^{i} \bar{E}, \wedge^{i} \bar{F}, \wedge^{i} \varphi_{K}\right)-h\left(\wedge^{i-1} \bar{E}, \wedge^{i-1} \bar{F}, \wedge^{i-1} \varphi_{K}\right) .
$$

\section{$\S$ B.4. Inégalités de pentes}

Soient $\bar{E}$ et $\bar{F}$ deux fibrés vectoriels hermitiens $\operatorname{sur} \operatorname{Spec} \mathcal{O}_{K}$ et $\varphi_{K}: E_{K} \rightarrow F_{K}$ une application $K$-linéaire.

Proposition B.4.1. Pour tout $j \in\left\{1, \ldots, \operatorname{rg} \varphi_{K}\right\}$, on a

$$
\begin{aligned}
& \widehat{\mu}_{\operatorname{dim} \operatorname{ker} \varphi_{K}+1}(\bar{E})+\cdots+\widehat{\mu}_{\operatorname{dim} \operatorname{ker} \varphi_{K}+j}(\bar{E}) \leq \\
& \widehat{\mu}_{1}(\bar{F})+\cdots+\widehat{\mu}_{j}(\bar{F})+\mu_{1}\left(\bar{E}, \bar{F}, \varphi_{K}\right)+\cdots+\mu_{j}\left(\bar{E}, \bar{F}, \varphi_{K}\right) .
\end{aligned}
$$

Proposition B.4.2. Pour tout $j \in\left\{1, \ldots, \operatorname{rg} \varphi_{K}\right\}$, on $a$

$$
\widehat{\mu}_{j+\operatorname{dim} \operatorname{ker} \varphi_{K}}(\bar{E}) \leq \widehat{\mu}_{j}(\bar{F})+h\left(\bar{E}, \bar{F}, \varphi_{K}\right) .
$$

Corollaire B.4.3. Si $\varphi_{K}$ est injective alors

$$
\widehat{\mu}_{\max }(\bar{E}) \leq \widehat{\mu}_{\max }(\bar{F})+h\left(\bar{E}, \bar{F}, \varphi_{K}\right) .
$$

On va maintenant généraliser ce corollaire de la facon suivante : ce n'est plus $F$ qui sera muni d'une structure hermitienne mais chacun des quotients associés à une filtration de $F$.

Soient donc $\bar{E}=(E,\|\|$.$) un fibré vectoriel hermitien sur \operatorname{Spec} \mathcal{O}_{K}$, et $F$ un fibré vectoriel $\operatorname{sur} \operatorname{Spec} \mathcal{O}_{K}$. Soit

$$
F=F^{0} \supseteq F^{1} \supseteq \cdots \supseteq F^{N}=\{0\}
$$

une filtration de $F$ par des sous-fibrés vectoriels (i.e. par des sous $\mathcal{O}_{K}$-modules tel que les $G^{i}=F^{i} / F^{i+1}$ soient sans torsion). On munit les $G^{i}(0 \leq i \leq N-1)$ d'une structure hermitienne $\|.\|_{i}$. Soit $\varphi_{K}: E_{K} \rightarrow F_{K}$ une application $K$-linéaire. On pose

$$
E_{K}^{i}:=\varphi_{K}^{-1}\left(F_{K}^{i}\right), E^{i}:=E \cap E_{K}^{i}
$$

et si on note $p^{i}$ la projection $F_{K}^{i} \rightarrow F_{K}^{i} / F_{K}^{i-1}, \varphi_{K}^{i}$ sera l'application composée de $p^{i}$ avec $\varphi_{K}$.

Théorème B.4.4. Si $\varphi_{K}: E_{K} \rightarrow F_{K}$ est injective alors

$$
\widehat{\operatorname{deg}}_{\mathrm{n}} \bar{E} \leq \sum_{i=0}^{N-1}\left(\operatorname{rg} E^{i}-\operatorname{rg} E^{i+1}\right)\left(\widehat{\mu}_{\max }\left(\bar{G}^{i}\right)+h\left(\bar{E}^{i}, \bar{G}^{i}, \varphi_{K}^{i}\right)\right)
$$

Démonstration rapide. On a

$$
\widehat{\operatorname{deg}}_{\mathrm{n}} \bar{E}=\sum_{i=0}^{N-1}\left(\widehat{\operatorname{deg}}_{\mathrm{n}} \bar{E}^{i}-\widehat{\operatorname{deg}}_{\mathrm{n}} \bar{E}^{i+1}\right)=\sum_{i=0}^{N-1} \widehat{\operatorname{deg}}_{\mathrm{n}}\left(\overline{E^{i} / E^{i+1}}\right)
$$

et

$$
E_{K}^{i} / E_{K}^{i+1} \hookrightarrow F_{K}^{i} / F_{K}^{i+1}
$$


donc d'après le corollaire

$$
\widehat{\mu}_{\max }\left(\overline{E^{i} / E^{i+1}}\right) \leq \widehat{\mu}_{\max }\left(\bar{G}^{i}\right)+h\left({\overline{E^{i} / E^{i+1}}}, \bar{G}^{i}, \varphi_{K}^{i}\right) .
$$

Il ne reste plus qu'à remarquer que

$$
h\left(\overline{E^{i} / E^{i+1}}, \bar{G}^{i}, \varphi_{K}^{i}\right) \leq h\left(\bar{E}^{i}, \bar{G}^{i}, \varphi_{K}^{i}\right) .
$$

\section{$\S$ B.5. Lien entre le corollaire, le lemme de Siegel et le théo- rème de Minkowski}

Pour la commoditée du lecteur, nous rappelons deux formes possibles du lemme de Siegel et du théorème de Minkowski.

Lemme de Siegel Soit $\left(a_{i, j}\right)_{\substack{1 \leq i \leq r \\ 1 \leq j \leq n}}$ une matrice rectangulaire à coefficients dans $\mathbb{Z}$ et soit $\varphi: \mathbb{Z}^{n} \rightarrow \mathbb{Z}^{r}$ le morphisme de $\mathbb{Z}$-module associé. On pose $A=\max _{i, j}\left\{\left|a_{i, j}\right|\right\}$. Alors, si $n>r$, il existe $x \in \operatorname{ker} \varphi \backslash\{0\}$ tel que

$$
\|x\| \leq(n A)^{\frac{r}{n-r}}
$$

où \|. || est la norme euclidienne.

Théorème de Minkowski Soit $\Gamma \subseteq \mathbb{R}^{n}$ un réseau, $\mathfrak{D} \subseteq \mathbb{R}^{n}$ un compact convexe symétrique. Alors, si vol $\mathfrak{D} \geq 2^{n} \operatorname{covol}(\Gamma)$, il existe $x \in \Gamma \cap \mathfrak{D} \backslash\{0\}$.

Nous allons démontrer le lemme de Siegel à partir du corollaire du paragraphe précédent et du théorème de Minkowski.

L'application $\widetilde{\varphi}: \mathbb{Z}^{n} / \operatorname{ker} \varphi \hookrightarrow \mathbb{Z}^{r}$ est injective donc par le corollaire B.4.3

$$
\widehat{\mu}_{\max }\left(\overline{\mathbb{Z}^{n} / \operatorname{ker} \varphi}\right) \leq h\left(\overline{\overline{\mathbb{Z}}^{n} / \operatorname{ker} \varphi}, \overline{\mathbb{Z}}^{r}, \widetilde{\varphi}\right)
$$

et donc

$$
-\widehat{\operatorname{deg}}_{\mathrm{n}} \overline{\operatorname{ker} \varphi} \leq \operatorname{rg}\left(\mathbb{Z}^{n} / \operatorname{ker} \varphi\right) \log \|\widetilde{\varphi}\| .
$$

Mais par ailleurs $\widehat{\operatorname{deg}}_{\mathrm{n}} \overline{\operatorname{ker} \varphi}=-\log (\operatorname{covol}(\operatorname{ker} \varphi),\|\|$.$) et \|\widetilde{\varphi}\| \leq\|\varphi\|$ donc

$$
\operatorname{covol}(\operatorname{ker} \varphi) \leq\|\varphi\|^{\operatorname{rg} \varphi} .
$$

D'après Minkowski, si $\operatorname{vol}(B(0, t)) \geq 2^{N} \operatorname{covol}(\operatorname{ker} \varphi)(N=n-\operatorname{rg} \varphi)$, donc en particulier, si $\operatorname{vol}(B(0, t)) \geq 2^{N}\|\varphi\|^{\operatorname{rg} \varphi}$, il existe $x \in \operatorname{ker} \varphi-\{0\}$ tel que $\|x\| \leq t$. Mais $\operatorname{vol}(B(0, t))=t^{N} \frac{\pi^{\frac{N}{2}}}{\Gamma\left(\frac{N}{2}+1\right)}$ donc

$$
t=\frac{2}{\sqrt{\pi}} \max _{1 \leq i \leq n}\left\{\Gamma(i / 2+1)^{1 / i}\right\} \times\|\varphi\|^{\frac{\mathrm{rg} \varphi}{n-\mathrm{rg} \varphi}}
$$

convient. Il suffit alors de voir que $\frac{\operatorname{rg} \varphi}{n-\operatorname{rg} \varphi} \leq \frac{r}{n-r}$ et que $\|\varphi\| \leq n A$.

Nous allons voir que l'application du théorème B.4.4 permet d'éviter l'emploi du lemme de Siegel (généralement utilisé pour construire les fonctions auxiliaires dans les démonstrations de transcendance) et de l'inégalité de Liouville. Deux autres outils habituels en transcendance - lemme de zéros et lemme de Schwarz - seront encore nécessaires ... 


\section{$\S$ B.6. Application à la transcendance : théorème des six exponentielles}

Il s'agit de démontrer (avec la méthode des pentes) le théorème suivant :

Théorème B.6.1. Soient $d, \ell$ des entiers naturels tels que $d \ell>d+\ell$ et soient $x_{1}, \ldots, x_{d}$ (resp. $\left.y_{1}, \ldots, y_{\ell}\right)$ des nombres complexes $\mathbb{Q}$-linéairement indépendants ( resp. idem). Alors l'un au moins des nombres $e^{x_{i} y_{j}}(1 \leq i \leq d, 1 \leq j \leq \ell)$ n'est pas algébrique sur $\mathbb{Q}$.

Ce théorème peut être formulé de manière équivalente en choisissant $(d, \ell)=(2,3)$ (ce qui fait 6 exponentielles !). La conjecture des quatre exponentielles concerne le cas limite $d=\ell=2$ :

Conjecture des 4 exponentielles : Soient $x_{1}, x_{2}, y_{1}, y_{2}$ des nombres complexes non nuls tels que ni $x_{1} / x_{2}$ ni $y_{1} / y_{2}$ n'appartiennent à $\mathbb{Q}$. Alors au moins un des 4 nombres $e^{x_{1} y_{1}}, e^{x_{1} y_{2}}, e^{x_{2} y_{1}}, e^{x_{2} y_{2}}$ est transcendant.

La démonstration 《 classique » de ce théorème consiste à construire un polynôme $P$ de $\mathbb{Z}\left[X_{1}, \ldots, X_{d}\right]$ de hauteur assez petite et tel que

$$
f(z):=P\left(e^{x_{1} z}, \ldots, e^{x_{d} z}\right)
$$

ne soit pas identiquement nul et s'annule en de «nombreux » points du groupe $\mathbb{Z} y_{1}+\cdots+\mathbb{Z} y_{\ell}$. On minore alors $\alpha=f\left(s_{1} y_{1}+\cdots+s_{\ell} y_{\ell}\right)$ par le théorème de Liouville lorsque $\alpha \neq 0$ et on majore ce nombre par le lemme de Schwarz. Une contradiction est alors censée apparaître. Ici le lemme de Liouville est remplacé par l'inégalité de pentes (B.2), qui permet un raisonnement plus directe et l'obtention de renseignements (bien faible au regard de la conjecture de Lehmer) en direction de la conjecture des 4 exponentielles (cf. § B.6.g). Commençons la démonstration du théorème B.6.1.

\section{$\S$ B.6.a. Données}

Nous raisonnons par l'absurde et nous considérons $K$ un corps de nombres contenant tous les $e^{x_{i} y_{j}}$. Le corps $K$ est un sous-corps de $\mathbb{C}$ et nous noterons $\sigma_{0}: K \hookrightarrow \mathbb{C}$ l'inclusion. Soient $L, S_{1} \leq S_{2}$ des entiers strictement positifs. On prendra

$$
E:=\left\{P \in \mathcal{O}_{K}\left[X_{1}, \ldots, X_{d}\right] ; \operatorname{deg}_{X_{i}} P \leq L-1\right\}, \quad F:=\mathcal{O}_{K}^{S_{2}^{\ell}}
$$

et $\varphi_{K}: E_{K} \rightarrow F_{K}$ l'application linéaire définie par

$$
\varphi_{K}(P)=\left(f\left(s_{1} y_{1}+\cdots+s_{\ell} y_{\ell}\right)\right)_{\substack{0 \leq s_{j} \leq S_{2}-1 \\ 1 \leq j \leq \ell}} .
$$

La filtration choisie est ici très simple puisqu'elle comporte trois termes seulement :

$$
F \supseteq F^{1} \supseteq F^{2}=\{0\}
$$

où

$$
F^{1}=\left\{\left(a_{s_{1}}, \ldots, s_{\ell}\right)_{0 \leq s_{i}<S_{2}} \in F ; a_{s_{1}, \ldots, s_{\ell}}=0 \quad \forall 0 \leq s_{i} \leq S_{1}-1\right\} .
$$


L'espace $E$ et les quotients $F^{i} / F^{i+1}$ sont munis des métriques hermitiennes : $\left(\sum_{i}\left|\sigma\left(x_{i}\right)\right|^{2}\right)^{\frac{1}{2}}$ $(\sigma: K \rightarrow \mathbb{C}$ est un plongement de $K$ dans $\mathbb{C})$. Enfin, $\varphi_{K}^{i}$ désigne l'application de $\varphi_{K}^{-1}\left(F_{K}^{i}\right) \rightarrow$ $F_{K}^{i} / F_{K}^{i+1}$, déduite de $\varphi_{K}$. De manière concrète, on a

$$
\begin{gathered}
\varphi_{K}^{0}(P)=\left(f\left(s_{1} y_{1}+\cdots+s_{\ell} y_{\ell}\right)\right)_{\substack{0 \leq s_{j} \leq S_{1}-1 \\
1 \leq j \leq \ell}} \text { et } P \in E_{K}, \\
\varphi_{K}^{1}(P)=\left(f\left(s_{1} y_{1}+\cdots+s_{\ell} y_{\ell}\right)\right)_{\substack{0 \leq s_{j} \leq S_{2}-1 \\
1 \leq j \leq \ell}}
\end{gathered}
$$

avec

$$
P \in E^{1}=\left\{P \in E ; f\left(s_{1} y_{1}+\cdots+s_{\ell} y_{\ell}\right)=0 \text { pour } 0 \leq s_{j} \leq S_{1}-1\right\}
$$

\section{$\S$ B.6.b. Injectivité de $\varphi_{K}$}

Nous utilisons le lemme de Tijdeman suivant, majorant le nombre de zéros d'un polynôme exponentiel dans le disque $D(0, R)$ (voir chapitre 6 de [79]) :

Lemme B.6.2. Si $h(z)=\sum_{i=1}^{n} a_{i}(z) e^{\omega_{i} z}$ où $a_{i} \in \mathbb{C}[X] \backslash\{0\}$ et $\omega_{1}, \ldots, \omega_{n}$ sont des nombres complexes deux à deux distincts alors le nombre de zéros de $h$ (avec multiplicité) dans $D(0, R)$ est inférieur à

$$
2\left(\operatorname{deg} a_{1}+\cdots+\operatorname{deg} a_{n}+n-1\right)+5 R \max _{i}\left\{\left|\omega_{i}\right|\right\} .
$$

Dans le cas présent, l'application $\varphi_{K}$ est injective dès que

$$
S_{2}^{\ell} \geq 2 L^{d}+5 L S_{2} \theta
$$

où $\theta:=\left(\left|x_{1}\right|+\cdots+\left|x_{d}\right|\right)\left(\left|y_{1}\right|+\cdots+\left|y_{\ell}\right|\right)$.

\section{$\S$ B.6.c. Évaluation de $\left\|\varphi_{K}^{i}\right\|_{\mathfrak{p}},\left\|\varphi_{K}^{i}\right\|_{\sigma}$}

Les majorations de cette section sont très larges et faciles à obtenir.

Soit $i \in\{0,1\}$. Pour toute place finie $\mathfrak{p}$ de $K$, on a

$$
\left\|\varphi_{K}^{i}\right\|_{\mathfrak{p}} \leq \max _{\substack{0 \leq \ell_{i} \leq L-1 \\ 0 \leq s_{i} \leq S-1}}\left\{\left|e^{\left(\ell_{1} x_{1}+\cdots+\ell_{d} x_{d}\right)\left(s_{1} y_{1}+\cdots+s_{\ell} y_{\ell}\right)}\right|_{\mathfrak{p}}\right\} \leq \alpha_{\mathfrak{p}}^{L S_{2}}
$$

où

$$
\alpha_{\mathfrak{p}}=\max \left(1,\left|e^{x_{1} y_{1}}\right|_{\mathfrak{p}}, \ldots,\left|e^{x_{d} y_{\ell}}\right|_{\mathfrak{p}}\right)
$$

De même, par Cauchy-Schwarz, pour toute place infinie $\sigma$,

$$
\begin{aligned}
\left\|\varphi_{K}^{i}(P)\right\|_{\sigma}^{2} & \leq S_{2}^{\ell}\left(\sum_{0 \leq \ell_{j} \leq L-1}\left|e^{\left(\ell_{1} x_{1}+\cdots+\ell_{d} x_{d}\right)\left(s_{1} y_{1}+\cdots+s_{\ell} y_{\ell}\right)}\right|_{\sigma}^{2}\right) \times\|P\|_{\sigma}^{2} \\
& \leq S_{2}^{\ell} L^{d} \alpha_{\sigma}^{2 L S_{2}}\|P\|_{\sigma}^{2}
\end{aligned}
$$

où

$$
\alpha_{\sigma}=\max \left(1,\left|e^{x_{1} y_{1}}\right|_{\sigma}, \ldots,\left|e^{x_{d} y_{\ell}}\right|_{\sigma}\right)
$$

D'où finalement :

$$
\left\|\varphi_{K}^{i}\right\|_{\sigma} \leq\left(S_{2}^{\ell} L^{d}\right)^{0.5} \alpha_{\sigma}^{L S_{2}}
$$




\section{$\S$ B.6.d. Majoration plus fine pour $\left\|\varphi_{K}^{1}\right\|_{\sigma_{0}}$}

Dans l'estimation précédente on n'a pas tenu compte du fait que $\varphi^{1}$ est évalué en $P \in E_{K}^{1}$ i.e. que

$$
P\left(e^{x_{1} z}, \ldots, e^{x_{d} z}\right)\left(s_{1} y_{1}+\cdots+s_{\ell} y_{\ell}\right)=0 \text { pour tout } 0 \leq s_{j} \leq S_{1}-1 .
$$

Pour cela on va utiliser la généralisation (bien connue) du lemme de Schwarz :

Lemme B.6.3. Soient $R>r, \rho \in[0, R]$, et $h$ une fonction holomorphe dans le disque $D(0, R)$ s'annulant en $N$ points distincts de $D(0, r)$. Alors, si on note $|h|_{x}=\sup _{|z| \leq x}|h(z)|$, on $a$

$$
|h|_{\rho} \leq\left(\frac{R(r+\rho)}{R^{2}+r \rho}\right)^{N}|h|_{R} .
$$

Prenons alors $P \in E_{K}^{1}$. On applique le lemme précédent à $h=f$ avec $r=S_{1}\left(\left|y_{1}\right|+\cdots+\left|y_{\ell}\right|\right)$, $\rho=S_{2}\left(\left|y_{1}\right|+\cdots+\left|y_{\ell}\right|\right), R=2 \rho$. On trouve

$$
\left\|\varphi_{K}^{1}(P)\right\|_{\sigma_{0}} \leq\left(L^{d} S_{2}^{\ell}\right)^{0.5}\|P\|_{\sigma_{0}}\left(\frac{2\left(S_{1}+S_{2}\right)}{4 S_{2}+S_{1}}\right)^{S_{1}^{\ell}} e^{2 L S_{2} \theta} .
$$

Comme $S_{1} \leq S_{2}$, on a

$$
\frac{2\left(S_{1}+S_{2}\right)}{4 S_{2}+S_{1}} \leq \frac{4}{5}
$$

dont nous déduisons finalement :

$$
\left\|\varphi_{K}^{1}\right\|_{\sigma_{0}} \leq\left(L^{d} S_{2}^{\ell}\right)^{0.5}\left(\frac{4}{5}\right)^{S_{1}^{\ell}} e^{2 L S_{2} \theta} .
$$

§ B.6.e. Évaluation de $\frac{\operatorname{rg} E-\operatorname{rg} E_{1}}{\operatorname{rg} E_{1}}$

Comme $E / E^{1}$ s'injecte dans $F / F^{1}$ on a

$$
\operatorname{rg} E-\operatorname{rg} E_{1} \leq \operatorname{rg} F-\operatorname{rg} F^{1}=S_{1}^{\ell} .
$$

Donc $\operatorname{rg} E^{1} \geq L^{d}-S_{1}^{\ell}$. Ainsi $\operatorname{rg} E^{1}$ est non nul et

$$
\frac{\operatorname{rg} E-\operatorname{rg} E^{1}}{\operatorname{rg} E^{1}} \leq 1
$$

dès que $L^{d} \geq 2 S_{1}^{\ell}$.

\section{§ B.6.f. On applique l'inégalité de pente}

En remarquant que $\widehat{\mu}_{\max }\left(\bar{G}^{i}\right)=0$ et $\widehat{\operatorname{deg}} \bar{E}=0$ (voir remarque de la fin du $\S$ B.2), l'inégalité (B.2) du théorème B.4.4 se ramène à

$$
\left(\operatorname{rg} E-\operatorname{rg} E^{1}\right) \times h\left(\varphi_{K}^{0}\right)+\operatorname{rg} E^{1} \times h\left(\varphi_{K}^{1}\right) \geq 0 .
$$


Les évaluations précédentes des normes des $\varphi^{i}$ donnent les majorations suivantes des hauteurs de ces morphismes :

$$
h\left(\varphi^{0}\right) \leq \frac{1}{2} \log \left(S_{2}^{\ell} L^{d}\right)+L S_{2} h\left(1: e^{x_{1} y_{1}}: \cdots: e^{x_{d} y_{\ell}}\right) .
$$

et

$$
h\left(\varphi^{1}\right) \leq \frac{1}{2} \log \left(S_{2}^{\ell} L^{d}\right)+L S_{2} h\left(1: e^{x_{1} y_{1}}: \cdots: e^{x_{d} y_{\ell}}\right)+\frac{2 L S_{2} \theta}{[K: \mathbb{Q}]}+\frac{S_{1}^{\ell}}{[K: \mathbb{Q}]} \log \left(\frac{4}{5}\right)
$$

Compte tenu du § B.6.e, l'inégalité (B.4) devient alors :

$$
\frac{1}{2} \log \left(S_{2}^{\ell} L^{d}\right)+L S_{2} h\left(1: e^{x_{1} y_{1}}: \cdots: e^{x_{d} y \ell}\right)+\frac{L S_{2} \theta}{[K: \mathbb{Q}]}+\frac{S_{1}^{\ell}}{2[K: \mathbb{Q}]} \log \left(\frac{4}{5}\right) \geq 0 .
$$

\section{$\S$ B.6.g. Conclusion}

Il ne reste plus qu'à choisir les paramètres pour obtenir une contradiction, sachant que doivent être satisfaites les trois conditions suivantes :

- $S_{2} \geq S_{1} \geq 1$

- $S_{2}^{\ell} \geq 2 L^{d}+5 L S_{2} \theta$ (lemme de zéros)

- $L^{d} \geq 2 S_{1}^{\ell}$

Pour cela choisissons $L=\left[2^{1 / d} S_{1}^{\ell / d}\right]+1$ et $S_{2}=\left[40 \max \{1, \theta\} S_{1}\right]+1$. On obtient alors

$$
\begin{aligned}
0 \leq 80 & \max \{1, \theta\} S_{1}^{1+\ell / d} \times\left(h\left(1: e^{x_{1} y_{1}}: \cdots: e^{x_{d} y_{\ell}}\right)+\frac{\theta}{[K: \mathbb{Q}]}\right)+\frac{S_{1}^{\ell}}{2[K: \mathbb{Q}]} \log \left(\frac{4}{5}\right) \\
& +\frac{1}{2} \log \left(80^{\ell} \max \{1, \theta\}^{\ell} S_{1}^{2 \ell}\right) .
\end{aligned}
$$

Si $d \ell>d+\ell$ (théorème des six exponentielles), la contradiction vient en faisant tendre $S_{1}$ vers l'infini.

Si $d \ell=d+\ell$ (i.e. $d=\ell=2$, la conjecture des quatres exponentielles), l'inégalité ci-dessus devient :

$$
\max \{1, \theta\}\left(h\left(1: e^{x_{1} y_{1}}: \cdots: e^{x_{d} y_{\ell}}\right)+\frac{\theta}{[K: \mathbb{Q}]}\right) \geq \frac{0.0001}{[K: \mathbb{Q}]}
$$

et donc si $\theta \leq 0.00005$, on a

$$
h\left(1: e^{x_{1} y_{1}}: \cdots: e^{x_{d} y_{\ell}}\right) \geq \frac{0.0001}{2[K: \mathbb{Q}]} .
$$

Malheureusement nous n'arrivons pas à obtenir de contradictions à partir de cette minoration (qui peut être raffinée au niveau des constantes). Du reste, les méthodes classiques (théorème de Liouville) peuvent donner de telles minorations, mais de manière plus compliquée. Car un des grands atouts de la méthode des pentes est sa simplicité de mise en œuvre alliée à une grande efficacité dans le domaine de l'approximation diophantienne. 
Annexe B. Inégalités de pentes et Transcendance 


\section{Annexe C}

\section{Aide-mémoire sur la notion de voisinage infinitésimal en géométrie algébrique}

\section{$\S$ C.1. Introduction}

Ce texte constitue une tentative de l'auteur pour comprendre les liens entre les différentes définitions possibles d'un voisinage infinitésimal en géométrie algébrique et l'intérêt que l'on peut y trouver pour formuler et résoudre (élégamment) des problèmes d'approximations diophantiennes. La notion de voisinage infinitésimal va de pair avec celle de schéma formel, qui sont les limites inductives de tels voisinages. Lorsque $\mathscr{X}$ est un $\mathbb{Z}$-schéma et $\varepsilon \in \mathscr{X}(\mathbb{Z})$ une section de $\mathscr{X}$, nous construisons le $n^{\text {ième }}$ voisinage infinitésimal $\mathscr{X}^{(n)}$ de $\mathscr{X}$ le long de $\varepsilon$. La limite inductive

$$
\widehat{\mathscr{X}_{\varepsilon}}:=\underset{n}{\lim _{\longrightarrow}} \mathscr{X}^{(n)}
$$

de ces schémas est un schéma formel qui rend compte des propriétés des sections de $\mathcal{O}_{\mathscr{X}}$ au voisinage de $\varepsilon$. En particulier, lorsque $\widehat{\mathscr{X}}$ est isomorphe (en tant que schéma formel) au spectre (formel) Specf $\mathbb{Z}\left[\left[X_{1}, \ldots, X_{g}\right]\right]$ d'une algèbre de séries formelles sur $\mathbb{Z}$, cela signifie qu'au voisinage $\varepsilon$, il existe un paramétrage qui permet d'exprimer les sections de $\mathcal{O}_{\mathscr{X}}$ comme des séries en ces paramètres locaux à coefficients dans $\mathbb{Z}$. Autrement dit, les coefficients de Taylor (les « dérivées divisées ») des sections de $\mathcal{O}_{\mathscr{X}}$ sont des entiers relatifs. L'isomorphisme de $\widehat{\mathscr{X}_{\varepsilon}}$ avec Specf $\mathbb{Z}\left[\left[X_{1}, \ldots, X_{g}\right]\right]$ s'obtient notamment lorsque $\mathscr{X} \rightarrow \mathbb{Z}$ est lisse en les points de $\varepsilon(\mathbb{Z})$. Ainsi, le concept de lissité est porteur d'une information de nature arithmétique sur les jets de sections. Cette remarque n'a guère été utilisée jusqu'à présent pour obtenir des mesures d'indépendances linéaires de logarithmes, sauf dans le cas des tores (commutatifs) via les polynômes de Fel'dman (voir Entracte, § II.5.d, page 85). Nous nous sommes (très largement) inspirés du tome IV des Éléments de Géométrie Algébrique de GROTHENDIECK [43], sans pour autant examiner le sujet ex professo !

\section{$\S$ C.2. Voisinages infinitésimaux}

Soit $S$ un schéma, $\pi: X \rightarrow S$ un schéma sur $S$ et $\varepsilon: S \rightarrow X$ une section de $X$. Soit $U$ un ouvert de $X$ tel que $\varepsilon$ se factorise en une immersion fermée $\widetilde{\varepsilon}: S \rightarrow U$ via le morphisme 
d'inclusion $i: U \rightarrow X$. On note $\mathcal{I}_{\varepsilon}=\operatorname{ker}\left(\mathcal{O}_{U} \rightarrow \widetilde{\varepsilon}_{*} \mathcal{O}_{S}\right)$ le faisceau d'idéaux définissant l'immersion fermée $\widetilde{\varepsilon}$.

Définition C.2.1. Le premier voisinage infinitésimal de $X$ le long de $\varepsilon$, que l'on note $S_{\varepsilon}^{(1)}$ ou $S^{(1)}$ s'il n'y a pas de confusion, est le sous-schéma fermé de $U$ défini par $\mathcal{I}_{\varepsilon}^{2}$. Plus généralement, le $n^{\text {ème }}$ voisinage infinitésimal de $X$ le long de $\varepsilon$, que l'on note $S_{\varepsilon}^{(n)}$ ou $S^{(n)}$, est le sous-schéma fermé* de $U$ défini par le faisceau d'idéaux $\mathcal{I}_{\varepsilon}^{n+1}$.

Le voisinage $S^{(n)}$ est indépendant du choix de l'ouvert $U$ (en question).

\section{Propriété C.2.2.}

1. Si $X$ est nœthérien alors $S^{(n)}$ est nœthérien.

2. Si $X / S$ est séparé alors $S^{(n)}$ est un sous-schéma de $X$.

3. Si $S$ est affine alors $S^{(n)}$ est affine.

Ces voisinages infinitésimaux sont l'analogue, en analyse, des développements limités tronqués à l'ordre $n$ d'une fonction. La section $\varepsilon$ joue le rôle du point en lequel on effectue le développement limité. On peut aussi considérer des dérivées et les voisinages associés globalement sur $X$ :

Définition C.2.3. Soit $\Delta_{\pi}: X \rightarrow X \times_{S} X$ l'immersion diagonale et $\mathcal{I}_{\Delta_{\pi}}$ le faisceaux d'idéaux correspondant. Le faisceau des parties principales d'ordre $n$ du $S$-schéma $X$, noté $\mathcal{P}_{X / S}^{n}$, est le faisceau d'anneaux $(\operatorname{sur} X): \Delta_{\pi}^{*}\left(\mathcal{O}_{X \times{ }_{S} X} / \mathcal{I}_{\Delta_{\pi}}^{n+1}\right)$.

L'espace annelé $\left(X, \mathcal{P}_{X / S}^{n}\right)$ s'identifie alors à $X_{\Delta_{\pi}}^{(n)}$. On désigne par $\mathrm{d}_{X / S}^{n}$ l'homomorphisme de $\mathcal{O}_{X} \rightarrow \mathcal{P}_{X / S}^{n}$ déduit de la projection sur le second facteur $p_{2}: X \times_{S} X \rightarrow X$ (via $\mathcal{O}_{X} \rightarrow$ $\left.p_{2 *} \mathcal{O}_{X \times_{S} X} \rightarrow p_{2 *}\left(\mathcal{O}_{X \times_{S} X} / \mathcal{I}_{\Delta_{\pi}}^{n+1}\right)\right)$. Intuitivement, c'est l'analogue de l'application qui à $f \in$ $\mathcal{C}^{\infty}(\mathbb{R}, \mathbb{R})$ associe le $(n+1)$-uplet de fonctions $\left(f, f^{\prime}, \cdots, \frac{1}{n !} f^{(n)}\right)$ car, modulo l'idéal $\mathcal{I}_{\Delta_{\pi}}^{n+1}$, on a

$$
f\left(z_{2}\right)=\sum_{k=0}^{n}\left(z_{2}-z_{1}\right)^{k} \frac{f^{(k)}\left(z_{1}\right)}{k !} .
$$

On verra plus loin que $\mathrm{d}_{X / S}^{n}$ est un opérateur différentiel.

Propriété C.2.4. Si $X / S$ est localement de type fini alors $\mathcal{P}_{X / S}^{n}$ est un $\mathcal{O}_{X}$-module quasicohérent de type fini.

Exemple C.2.5. Si $R$ est un anneau alors

$$
\mathcal{P}_{\mathbb{A}^{g} / R}^{n} \stackrel{\sim}{\rightarrow} \frac{R\left[X_{1}, \ldots, X_{g}, Y_{1}, \ldots, Y_{g}\right]}{\left(Y_{1}, \ldots, Y_{g}\right)^{n+1}}
$$

${ }^{*}$ En fait l'espace topologique sous-jacent à $S_{\varepsilon}^{(n)}$, noté $S_{\varepsilon \text {, top }}^{(n)}$, est $S_{\text {top }}$ car $S_{\varepsilon \text {, top }}^{(n)}$ est naturellement isomorphe au support de $\mathcal{O}_{U} / I^{n+1}\left(=\left\{x \in U ;\left(\mathcal{O}_{U} / I^{n+1}\right)_{x} \neq 0\right\}\right)$, qui est aussi le support de $\mathcal{O}_{U} / I$, i.e. $S_{\text {top }}$. 
Ainsi se donner un élément de $\mathcal{P}_{\mathbb{A}^{g} / R}^{n}$ (i.e. une section globale de ce fibré) revient à se donner des polynômes $\left(\left(\begin{array}{c}g+n \\ n\end{array}\right)\right.$ exactement) de $R\left[X_{1}, \ldots, X_{g}\right]$, qui forment une famille de coefficients de Taylor jusqu'à l'ordre $n$.

Il existe une autre définition des voisinages infinitésimaux $S_{\varepsilon}^{(n)}$. Considérons l'immersion fermée $\widetilde{\varepsilon}: S \rightarrow U$ déduite de $\varepsilon$ (voir le début de ce paragraphe). L'homomorphisme surjectif $\widetilde{\varepsilon}^{*} \mathcal{O}_{U} \rightarrow \mathcal{O}_{S}$ induit un isomorphisme

$$
\widetilde{\varepsilon}^{*} \mathcal{O}_{U} / \mathcal{J}_{\widetilde{\varepsilon}} \stackrel{\sim}{\rightarrow} \mathcal{O}_{S}
$$

où $\mathcal{J}_{\widetilde{\varepsilon}}=\operatorname{ker}\left(\widetilde{\varepsilon}^{*} \mathcal{O}_{U} \rightarrow \mathcal{O}_{S}\right)$.

Définition C.2.6. L'espace annelé

$$
S_{\varepsilon}^{(n)}=\left(S,\left(\widetilde{\varepsilon}^{*} \mathcal{O}_{U}\right) / \mathcal{J}_{\widetilde{\varepsilon}}^{n+1}\right)
$$

est le $n^{\text {ème }}$ voisinage infinitésimal de $X$ le long de $\varepsilon$.

Son faisceau structural, $\mathcal{O}_{S_{\varepsilon}^{(n)}}=\widetilde{\varepsilon}^{*} \mathcal{O}_{U} / \mathcal{J}_{\widetilde{\varepsilon}}^{n+1}$, est appelé le $n^{\grave{e ̀ m e}}$ invariant normal de $\varepsilon$.

Proposition C.2.7. Avec les notations précédentes, on dispose d'un isomorphisme de $\mathcal{O}_{S^{-}}$ algèbres :

$$
\mathcal{O}_{S_{\varepsilon}^{(n)}} \stackrel{\sim}{\rightarrow} \varepsilon^{*} \mathcal{O}_{X_{\Delta_{\pi}}^{(n)}}
$$

et cet isomorphisme est unique si on demande la commutativité du diagramme

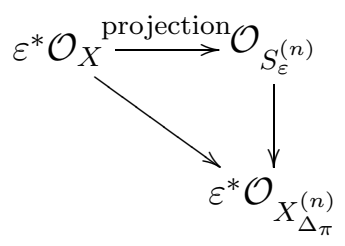

Cet isomorphisme traduit le fait que pour obtenir le développement limité à l'ordre $n$ au point $\varepsilon$, on peut d'abord dériver jusqu'à l'ordre $n\left(c^{\prime}\right.$ 'est $\left.\mathcal{O}_{X_{\Delta_{\pi}}^{(n)}}\right)$ puis spécifier au point $\varepsilon$ (d'où l'image inverse par $\varepsilon$ ).

\section{$\S$ C.2.a. Schémas et groupes formels}

On dispose d'homorphismes de projection $\varphi_{n m}: \mathcal{O}_{S^{(m)}} \rightarrow \mathcal{O}_{S^{(n)}}$ pour $n \leq m$ permettant d'identifier $\mathcal{O}_{S^{(n)}}$ avec le quotient de $\mathcal{O}_{S^{(m)}}$ par

$$
\left(\mathcal{I}_{\varepsilon} / \mathcal{I}_{\varepsilon}^{m+1}\right)^{n+1}
$$

Le système $\left(\mathcal{O}_{S^{(n)}}, \varphi_{n m}\right)$ est un système projectif de faisceaux d'anneaux sur $S$ dont la limite projective, parfois notée $\mathcal{O}_{S(\infty)}$, est appelée invariant normal d'ordre infini de $\varepsilon$. Lorsque $X / S$ est nœthérien et séparé, l'immersion $\varepsilon$ est fermée et $\mathcal{O}_{S(\infty)}$ n'est autre que le complété (formel) de $\mathcal{O}_{X}$ le long de $\varepsilon$. L'espace $\left(S^{(\infty)}, \mathcal{O}_{S(\infty)}\right)$ est le schéma formel complété de $X$ le long de $\varepsilon$, que l'on appelle aussi le voisinage formel de $S$ dans $X$ (le long de $\varepsilon$ ). Il peut être construit par limite inductive des voisinages infinitésimaux d'ordre $n$. Par exemple, si la situation de départ se compose d'un schéma en groupes $\pi: \mathbb{G} \rightarrow S$ séparé et de la $S$-section 
neutre $\varepsilon: S \rightarrow \mathbb{G}$, on peut compléter $\mathbb{G}$ le long de $\varepsilon$ pour obtenir le schéma formel $\widehat{\mathbb{G}}_{\varepsilon}$. Ce schéma formel a une structure de groupe formel car le morphisme d'addition du schéma en groupes $\mathbb{G}$ se prolonge à $\widehat{\mathbb{G}}_{\varepsilon}$. Le voisinage infinitésimal $S_{\varepsilon}^{(n)}$ s'identifie au $n^{\text {ème }}$ voisinage de $\widehat{\mathbb{G}}_{\varepsilon}$. L'avantage est que sous certaines hypothèses (par exemple la lissité), le groupe formel est isomorphe, en tant que schéma formel, à $\widehat{\mathbb{A}}^{n} \widehat{\times}_{\mathbb{Z}} S=\operatorname{Specf} \mathcal{O}_{S}\left[\left[X_{1}, \ldots, X_{n}\right]\right]$ (le produit $\widehat{\times}$ est celui de la catégorie des schémas formels, qui tient compte du fait que $\mathbb{Z}[[X]] \otimes \mathbb{Q} \neq \mathbb{Q}[[X]])$. L'entier $n$ est la dimension de $\mathbb{G} / S$ au point $\varepsilon$ (voir proposition C.3.11, page 118). Dans ce cas et lorsque $S=\operatorname{Spec} R$ est affine, la structure de groupe formel de $\widehat{\mathbb{G}}_{\varepsilon}$ se lit alors dans $\widehat{\mathbb{A}}^{n} \widehat{\times} S$, via la donnée d'un $n$-uplet de séries formelles $F=\left(F_{1}, \ldots, F_{n}\right)$ (appelée loi formelle), à coefficients dans $R$, en deux multivariables $\mathbf{X}=\left(X_{1}, \ldots, X_{n}\right)$ et $\mathbf{Y}=\left(Y_{1}, \ldots, Y_{n}\right)$, qui a les propriétés suivantes (héritées en droite ligne des propriétés de la loi de groupe de $\mathbb{G}$ ) :

- $F(F(\mathbf{X}, \mathbf{Y}), \mathbf{Z})=F(\mathbf{X}, F(\mathbf{Y}, \mathbf{Z}))$ (associativité)

$-F(0, \mathbf{X})=\mathbf{X}$ (élément neutre)

Si le schéma en groupes $\mathbb{G}$ est commutatif, on a également $F(\mathbf{X}, \mathbf{Y})=F(\mathbf{Y}, \mathbf{X})$. Ces propriétés impliquent que $F(\mathbf{X}, \mathbf{Y})=\mathbf{X}+\mathbf{Y} \bmod (\mathbf{X}, \mathbf{Y})^{2}$, égalité qui a son tour donne l'existence (et l'unicité) de la série inverse $i(\mathbf{X})$ vérifiant $F(\mathbf{X}, i(\mathbf{X}))=0$. Nous noterons parfois $\mathbf{X} \oplus_{\widehat{G}_{\varepsilon}} \mathbf{Y}$ au lieu de $F(\mathbf{X}, \mathbf{Y})$. Si $\varepsilon$ est la section nulle du schéma en groupes considéré, on a par exemple $\mathbf{X} \oplus_{\widehat{\mathbb{G}}_{\mathrm{a}, \varepsilon}} \mathbf{Y}=\mathbf{X}+\mathbf{Y}$ et $\mathbf{X} \oplus_{\widehat{\mathbb{G}}_{\mathrm{m}, \varepsilon}} \mathbf{Y}=\mathbf{X}+\mathbf{Y}+\mathbf{X Y}$.

Nous appellerons logarithme de $\widehat{\mathbb{G}}_{\varepsilon}$ un homomorphisme de $\widehat{\mathbb{G}}_{\varepsilon} \rightarrow \widehat{\mathbb{G}}_{\mathrm{a}}^{n}$ tangent à l'identité, ou de manière équivalente, la donnée d'un $n$-uplet de séries formelles $\ell(\mathbf{X}) \in(R[[\mathbf{X}]])^{n}$ vérifiant $\ell\left(\mathbf{X} \oplus_{\widehat{\mathbb{G}}_{\varepsilon}} \mathbf{Y}\right)=\ell(\mathbf{X})+\ell(\mathbf{Y})$ et $\ell(\mathbf{X})=\mathbf{X}+$ termes d'ordre $\geq 2$. Il peut ne pas en exister. Cependant, lorsque $R$ est une $\mathbb{Q}$-algèbre (hypothèse que nous ferons jusqu'à la fin de ce paragraphe), il existe un logarithme de $\widehat{\mathbb{G}}_{\varepsilon}$ dont les composantes proviennent de l'intégration (formelle) de formes différentielles invariantes :

Définition C.2.8. Une forme différentielle $\omega$ sur $\widehat{\mathbb{G}}_{\varepsilon}$ est invariante (par translation) si elle s'écrit

$$
\omega=\sum_{i=1}^{n} \widetilde{\omega}_{i}\left(X_{1}, \ldots, X_{n}\right) \mathrm{d} X_{i}
$$

avec $\widetilde{\omega}_{i} \in R[[\mathbf{X}]]$ et $\omega\left(\mathbf{X} \oplus_{\widehat{\mathbb{G}}_{\varepsilon}} \mathbf{Y}\right)=\omega(\mathbf{X})$.

Proposition C.2.9. Le R-module des formes différentielles invariantes est libre de rang $n$. De plus, $\left(\omega_{1}, \ldots, \omega_{n}\right)$ est une base de ce module si et seulement si $\ell(\mathbf{X})=\left(\int \omega_{1}, \ldots, \int \omega_{n}\right)$ est un logarithme de $\widehat{\mathbb{G}}_{\varepsilon}$.

Théorème C.2.10. Un logarithme de $\widehat{\mathbb{G}}_{\varepsilon}$ est un isomorphisme de groupes formels ; autrement dit tout groupe formel sur une $\mathbb{Q}$-algèbre est isomorphe, en tant que groupe formel, à une puissance du groupe formel additif. De plus, si on note $t_{\mathbb{G} / S}$ le dual de $\varepsilon^{*} \Omega_{\mathbb{G} / S}^{1}$ (qui est un quotient de $\left.\mathcal{I}_{\varepsilon} / \mathcal{I}_{\varepsilon}^{2}\right)$, alors il existe un isomorphisme canonique entre $\widehat{\mathbb{G}}_{\varepsilon}$ et le complété formel $\widehat{t}_{\mathbb{G} / S}$ de $t_{\mathbb{G} / S}$ par rapport à $\mathcal{I}$.

Ce théorème et cette proposition sont démontrés dans [44], p. 64.

D'après ce théorème, il existe, pour tout $n \in \mathbb{N} \backslash\{0\}$, un isomorphisme de schémas entre $\widehat{\mathbb{G}}_{\varepsilon}^{(n)}$ (qui gère les variations infinitésimales (d'ordre $n$ au voisinage de $\varepsilon$ ) directement dans le groupe $\mathbb{G}$ ) et $\widehat{t}_{\mathbb{G} / S}^{(n)}$ (qui gère les mêmes variations, mais dans l'espace tangent). Nous allons voir dans le paragraphe suivant une construction équivalente de $\widehat{t}_{\mathbb{G} / S}^{(n)}$ obtenue avec le fibré tangent. 


\section{$\S$ C.2.b. Cas d'un fibré vectoriel}

Lorsque $X$ est un fibré vectoriel associé à une $\mathcal{O}_{S^{-}}$algèbre, on peut préciser le faisceau des parties principales d'ordre $n$ et les voisinages infinitésimaux correspondant.

Soit $S$ un schéma, $\mathcal{E}$ un $\mathcal{O}_{S}$-module quasi-cohérent. On note $X=\mathbb{V}(\mathcal{E}):=\operatorname{Spec}(\mathbf{S}(\mathcal{E}))$ et $\pi: X \rightarrow S$ son morphisme structural $(\mathbf{S}(\mathcal{E})$ est l'algèbre symétrique de $\mathcal{E}$ ). Soit $\varepsilon: S \rightarrow X$ la $S$-section provenant de l'augmentation $\mathbf{S}(\mathcal{E}) \rightarrow \mathcal{O}_{S}$ et $\mathcal{I}=\operatorname{ker}\left(\mathbf{S}(\mathcal{E}) \rightarrow \mathcal{O}_{S}\right)$ son idéal de définition. Lorsque $\mathcal{E}$ est un $\mathcal{O}_{S}$-module (quasi-cohérent) de type fini, le schéma $X$ est aussi de type fini sur $S$ (et réciproquement).

Proposition C.2.11 (Proposition 16.4.8 de [43]). On dispose d'isomorphismes

$$
\delta_{n}: \pi^{*}\left(\mathbf{S}(\mathcal{E}) / \mathcal{I}^{n+1}\right) \stackrel{\sim}{\rightarrow} \mathcal{P}_{X / S}^{n}
$$

qui forment un système projectif et d'un isomorphisme de $\mathcal{O}_{X}$-algèbres graduées :

$$
\pi^{*}(\mathbf{S}(\mathcal{E})) \stackrel{\sim}{\rightarrow} \mathscr{G}_{\bullet}\left(\mathcal{P}_{X / S}\right):=\underset{n \geq 0}{\oplus}\left(\mathcal{I}_{\Delta_{\pi}}^{n} / \mathcal{I}_{\Delta_{\pi}}^{n+1}\right) .
$$

En particulier on a un isomorphisme canonique $\pi^{*} \mathcal{E} \stackrel{\sim}{\rightarrow} \Omega_{X / S}^{1}$.

Si on joint cette proposition à la proposition C.2.7, on déduit immédiatement que $\mathcal{O}_{S_{\varepsilon}^{(n)}} \stackrel{\sim}{\rightarrow}$ $\mathbf{S}(\mathcal{E}) / \mathcal{I}^{n+1}$ et donc $\mathcal{O}_{S_{\varepsilon}^{(n)}} \stackrel{\sim}{\rightarrow} \mathcal{O}_{S} \oplus \mathcal{E} \oplus \cdots \oplus S^{n}(\mathcal{E})$ en munissant ce dernier ensemble de la structure d'algèbre « quotient» induite par $\mathbf{S}(\mathcal{E}) / \mathcal{I}^{n+1}$ (le produit est l'exacte analogue du produit de deux développements limités d'ordre $n$ ). Ceci suggère une (troisième) définition (équivalente) pour le $n^{\text {ème }}$ voisinage $S_{\varepsilon}^{(n)}$ :

$$
S_{\varepsilon}^{(n)}:=\operatorname{Spec}\left(\mathcal{O}_{S} \oplus \mathcal{E} \oplus \cdots \oplus S^{n}(\mathcal{E})\right) .
$$

Le voisinage formel de $S$ dans $X$ n'est alors rien d'autre que le complété formel le long de $\varepsilon$ du fibré vectoriel $X$. Cette définition est particulièrement intéressante lorsqu'on veut dériver dans certaines directions privilégiées et non selon toutes les directions au «point » $\varepsilon$.

Avec les notations initiales ( $X$ schéma quelconque au-dessus de $S$ ), considérons le $\mathcal{O}_{X^{-}}$ module des différentielles $\Omega_{X / S}^{1}$ de $X / S$ et $t_{X / S, \varepsilon}=\operatorname{Hom}_{\mathcal{O}_{S}}\left(\varepsilon^{*} \Omega_{X / S}^{1}, \mathcal{O}_{S}\right)$ l'espace tangent à $X$ au point $\varepsilon$. On suppose que $\varepsilon^{*} \Omega_{X / S}^{1}$ est localement libre. Soit $\mathcal{W}$ un sous $\mathcal{O}_{S}$-module de $t_{X / S}$, localement facteur direct dans $t_{X / S}$, et soit $S_{\mathcal{W}}:=\mathbb{V}\left(\mathcal{W}^{\vee}\right)$. L'injection $\mathcal{W} \hookrightarrow t_{X / S}$ induit une surjection sur les duaux et donc une immersion fermée du fibré vectoriel $S_{\mathcal{W}}$ dans le fibré tangent* sur $S: \mathbb{T}_{X / S} \times_{X} S$. De plus ces deux fibrés sont des schémas affines au-dessus de $S$ et sont donc affines si $S$ est affine. Le fait est que le cotangent de $S_{\mathcal{W}}$ sur $S$ s'identifie au dual de $\mathcal{W}$. En effet, d'après la proposition C.2.11, il existe un isomorphisme canonique

$$
\pi^{*}\left(\mathcal{W}^{\vee}\right) \stackrel{\sim}{\rightarrow} \Omega_{S_{\mathcal{W}} / S}^{1}
$$

où $\pi: S_{\mathcal{W}} \rightarrow S$ est le morphisme structural. Par conséquent, si $\varepsilon_{\mathcal{W}}: S \rightarrow S_{\mathcal{W}}$ est la $S$-section de $\pi$ provenant du morphisme d'augmentation $\mathbf{S}\left(\mathcal{W}^{\vee}\right) \rightarrow \mathcal{O}_{S}$, on obtient $\mathcal{W}^{\vee} \stackrel{\sim}{\rightarrow} \varepsilon_{\mathcal{W}}^{*} \Omega_{S_{\mathcal{W}} / S}^{1}$. De la même manière, le sous-espace $\mathcal{W}$ est le tangent de chacun des voisinages

$$
S_{\mathcal{W}}^{(n)}=\operatorname{Spec}\left(\mathcal{O}_{S} \oplus \mathcal{W}^{\vee} \oplus \cdots \oplus S^{n}\left(\mathcal{W}^{\vee}\right)\right)
$$

${ }^{*}$ qui « moralement » s'identifie à $\operatorname{Lie}(X / S), c f$. [42] pages 49 et 74. 
Exemple C.2.12. Considérons $X=\mathbb{G}_{\mathrm{a}} \times \mathbb{G}_{\mathrm{m}}$ et $S=$ Spec $\mathbb{Z}$. Alors

$$
\begin{gathered}
\Omega_{X / S}^{1}=\mathbb{Z}\left[X, Y, Y^{-1}\right] \mathrm{d} X \oplus \mathbb{Z}\left[X, Y, Y^{-1}\right] \frac{\mathrm{d} Y}{Y}, \\
\varepsilon^{*} \Omega_{X / S}^{1}=\mathbb{Z} \mathrm{d} X \oplus \mathbb{Z} \frac{\mathrm{d} Y}{Y}, \\
t_{X / S}=\mathbb{Z} \frac{\partial}{\partial X} \oplus \mathbb{Z} Y \frac{\partial}{\partial Y} .
\end{gathered}
$$

Soit $(a, b) \in \mathbb{Z}^{2} \backslash\{0\}$ un couple d'entiers premiers entre eux, définissant l'hyperplan $\mathcal{W}$ de $t_{X / S}$ :

$$
z=x \frac{\partial}{\partial X}+y Y \frac{\partial}{\partial Y} \in \mathcal{W} \Longleftrightarrow a x+b y=0 .
$$

A partir de là, on aurait envie de définir $S_{\mathcal{W}}$ tout simplement comme $\operatorname{Spec}\left(\frac{\mathbb{Z}[x, y]}{(a x+b y)}\right)$. Eh bien c'est possible ! ... et on reste cohérent (à isomorphisme près) avec la première définition de $S_{\mathcal{W}}$. L'argument est le suivant. Comme $S_{\mathcal{W}}$ est affine (car affine au-dessus du schéma affine Spec $\mathbb{Z}$ ), il suffit de comparer leurs faisceaux structuraux. Or on a un isomorphisme de $\mathbb{Z}$-modules

$$
\frac{\mathbb{Z} \cdot x \oplus \mathbb{Z} \cdot y}{\mathbb{Z} \cdot(a x+b y)} \simeq \mathcal{W}^{\vee}
$$

donné par $(x, y) \rightarrow\left(\varphi_{(x, y)}: u \frac{\partial}{\partial X}+v Y \frac{\partial}{\partial Y} \mapsto u x+v y\right)$. L'isomorphisme de schémas voulu en découle alors immédiatement .

Sur cet exemple on voit que $S_{\mathcal{W}}^{(n)}$ gère les variations infinitésimales d'ordre $n$ dans la direction de $\mathcal{W}$ au niveau de l'espace tangent. En fait, il semble qu'il soit parfois plus efficace de considérer les variations infinitésimales directement au niveau du groupe $X$. Le «problème » qui se pose alors est que l'hyperplan vu dans $X$ n'est plus un schéma algébrique (par exemple ici c'est $a X+b \log (Y+1)=0$ qui n'est pas une équation polynomiale). On contournera le problème en considérant le voisinage d'ordre $n$ sur la fibre générique * et le schéma formel qui est leur limite inductive.

\section{§ C.2.c. Cas d'un schéma en groupes}

Lorsque $S$ est le spectre premier d'un anneau $R$ qui peut se plonger dans $\mathbb{C}$, il existe une manière plus « concrète » de définir le $n^{\text {ème }}$ voisinage infinitésimal $S_{\mathcal{W}}^{(n)}$ d'un schéma en groupes $\mathbb{G}$ le long d'une $S$-section $\varepsilon$ de $\mathbb{G}$, dans la direction d'un sous-espace vectoriel $\mathcal{W}$ de l'espace tangent $t_{\mathbb{G} / S, \varepsilon}=\left(\varepsilon^{*} \Omega_{\mathbb{G} / S}^{(1)}\right)^{\vee}$.

Soit $\sigma: R \rightarrow \mathbb{C}$ un plongement de $R$ dans $\mathbb{C}$. Notons $e_{\sigma}: t_{\mathbb{G}_{\sigma}(\mathbb{C})} \rightarrow \mathbb{G}_{\sigma}(\mathbb{C})$ l'exponentielle du groupe de Lie complexe $\mathbb{G}_{\sigma}(\mathbb{C})$. Le fibré $e_{\sigma}^{*} \mathcal{O}_{\mathbb{G}_{\sigma}}$ est le fibré trivial sur $t_{\mathbb{G}_{\sigma}(\mathbb{C})}$. Le choix d'une trivialisation de ce fibré (i.e. d'un isomorphisme $e_{\sigma}^{*} \mathcal{O}_{\mathbb{G}_{\sigma}} \stackrel{\sim}{\longrightarrow} t_{\mathbb{G}_{\sigma}(\mathbb{C})} \times \mathbb{C}$ ) permet d'associer bijectivement à une section $s$ de $\mathcal{O}_{\mathbb{G}_{\sigma}(\mathbb{C})}$ au-dessus d'un ouvert $U$ de $\mathbb{G}_{\sigma}(\mathbb{C})$ une fonction holomorphe $f$ sur $e_{\sigma}^{-1}(U)$. Soit $n$ un entier naturel et posons $h:=\operatorname{dim}\left(\mathcal{W} \otimes_{\sigma} \mathbb{C}\right)$. Considérons

*Sur l'exemple c'est : $\operatorname{Spec}\left(\frac{\mathbb{Q}[[X, Y]]}{\left(X^{n+1}\right)+\left(Y^{n+1}\right)+\left(a X+b\left(Y-\frac{1}{2} Y^{2}+\cdots+\frac{(-1)^{n-1}}{n} Y^{n}\right)\right)}\right)$. 
l'ensemble des sections $s$ de $\mathcal{O}_{\mathbb{G}_{\sigma}(\mathbb{C})}$, au-dessus d'un ouvert $U$ contenant le point $\varepsilon_{\sigma} \in \mathbb{G}_{\sigma}(\mathbb{C})$ (point obtenu par composition de $\varepsilon: \operatorname{Spec} R \rightarrow \mathbb{G}$ et de $\operatorname{Spec} \sigma: \operatorname{Spec} \mathbb{C} \rightarrow \operatorname{Spec} R$ ), dont la fonction holomorphe $f$ associée vérifie :

$$
f\left(u+z_{1} w_{1}+\cdots+z_{h} w_{h}\right) \in\left(z_{1}, \ldots, z_{h}\right)^{n+1}
$$

où $u$ est un logarithme de $\varepsilon_{\sigma}\left(e_{\sigma}(u)=\varepsilon_{\sigma}\right)$ et $\left(w_{1}, \ldots, w_{h}\right)$ une base de $\mathcal{W}_{\sigma}$. On vérifie que cette définition est indépendante du choix du logarithme de $\varepsilon_{\sigma}$, de la base de $\mathcal{W}_{\sigma}$ et de la trivialisation de $e_{\sigma}^{*} \mathcal{O}_{\mathbb{G}_{\sigma}}$. La condition (C.1) équivaut à se donner une base $\mathcal{D}_{1}, \ldots, \mathcal{D}_{h}$ de $\mathcal{W}_{\sigma}$ (les éléments de cette base sont vus ici comme des dérivations) et à imposer, pour tout $|\underline{k}| \leq n$,

$$
\left(\mathcal{D}_{1}^{k_{1}} \cdots \mathcal{D}_{h}^{k_{h}} f\right)(u)=0
$$

L'ensemble des sections $s$ ci-dessus forme un faisceau d'idéaux $\mathcal{I}_{\sigma, n}$ de $\mathcal{O}_{\mathbb{G}_{\sigma}(\mathbb{C})}$. Notons $\mathbb{G}_{\mathcal{W}}^{(n)}$ le sous-schéma fermé de $\mathbb{G}$ défini par le faisceau d'idéaux $\mathcal{I}_{\sigma, n} \cap \mathcal{O}_{\mathbb{G}}$ (le schéma $\mathbb{G}_{\mathcal{W}}^{(n)}$ est l'adhérence schématique dans $\mathbb{G}$ de $\left.S_{W_{\sigma}}^{(n)}\right)$. Ce sous-schéma fermé est affine. En effet, l'idéal $\mathcal{I}_{\sigma, n}$ contient $\mathcal{I}_{\sigma}^{n+1}\left(\mathcal{I}_{\sigma}=\operatorname{ker}\left(\mathcal{O}_{\mathbb{G}_{\sigma}} \rightarrow \varepsilon_{\sigma *} \mathcal{O}_{\text {Spec } \mathbb{C}}\right)\right)$ et donc la flèche $\mathbb{G}_{\mathcal{W}}^{(n)} \hookrightarrow S_{\varepsilon}^{(n)}$ est une immersion fermée de $\mathbb{G}_{\mathcal{W}}^{(n)}$ dans un schéma affine. Malheureusement, en général, $\mathbb{G}_{\mathcal{W}}^{(n)}$ n'est pas isomorphe à $S_{\mathcal{W}}^{(n)}$ car, comme nous le verrons plus loin (proposition C.4.1), le schéma formel $\underset{\lim }{\longrightarrow} S_{\mathcal{W}}^{(n)}$ (qui est aussi $\left.\widehat{\mathbb{V}\left(\mathcal{W}^{v}\right)}\right)$ est lisse sur $S$, contrairement (en général) à $\lim \mathbb{G}_{\mathcal{W}}^{(n)}$. Mais, par définition de l'adhérence schématique, nous disposons néanmoins d'une application $\mathbb{G}_{\mathcal{W}}^{(n)} \hookrightarrow S_{\mathcal{W}}^{(n)}$ qui est injective (car c'est un isomorphisme sur les fibres génériques).

Remarque : L'obstruction dans la non-lissité du schéma formel $\lim \mathbb{G}_{\mathcal{W}}^{(n)}$ peut être comparée à celle qui sépare la $n^{\text {ième }}$ puissance symbolique $I^{(n)}$ d'un idéal $I(\subseteq R)$ de la puissance $n^{i e ̀ m e}, I^{n}$, de $I$. Pour comprendre ce point, rappelons que $I^{(n)}$ est l'intersection des composantes primaires de $I^{n}$ associées aux idéaux premiers minimaux de $I^{n}$. Par exemple, si $\mathfrak{P}$ est un idéal premier de $R$ et si $I$ est un idéal $\mathfrak{P}$-primaire, $I^{(n)}$ est l'ensemble des éléments $x$ de $R$ pour lesquels il existe un élément $r$ de $R \backslash \mathfrak{P}$ tel que $r x$ appartienne à $I^{n}$. Pour $\mathfrak{P}$ lui-même, nous avons $\mathfrak{P}^{(n)}=\mathfrak{P} R_{\mathfrak{P}} \cap R\left(R_{\mathfrak{P}}\right.$ est l'anneau local en $\mathfrak{P}$, voir exercice 4.2 de [56], page 29). Dans le cas particulier où $I$ est un idéal radical $(I=\sqrt{I})$ d'un anneau de polynôme $R:=k\left[X_{1}, \ldots, X_{\ell}\right]$, sur un corps $k$ de caractéristique nulle, la puissance symbolique $I^{(n)}$ est

$$
\left\{P \in R ; \forall\left(\alpha_{1}, \ldots, \alpha_{\ell}\right), \alpha_{1}+\cdots+\alpha_{\ell} \leq n-1, \quad \frac{\partial^{\underline{\alpha}} P}{\partial X^{\underline{\alpha}}} \in I\right\} .
$$

Le lien avec la notion de lissité s'effectue via la notion de suite régulière. En effet, nous avons le

Théorème [Proposition 3.5.12 de [77], p. 95] : Si R est un anneau de Cohen-Macaulay et si $\mathfrak{P}$ est un idéal premier de $R$ localement engendré par une suite régulière de $\operatorname{dim} R_{\mathfrak{P}}$ éléments alors, pour tout entier $r \geq 1$, on a $\mathfrak{P}^{(r)}=\mathfrak{P}^{r}$.

Lorsque nous sommes comme ici avec un schéma en groupes, il est possible, modulo une hypothèse supplémentaire (lissité), de donner une caractérisation de $S_{\varepsilon}^{(n)}\left(\operatorname{resp} . S_{\mathcal{W}}^{(n)}\right)$ à partir du premier voisinage $S_{\varepsilon}^{(1)}\left(\operatorname{resp} . S_{\mathcal{W}}^{(1)}\right)$.

Soit $R$ une $\mathbb{Q}$-algèbre (commutative) et $S=\operatorname{Spec} R$. Soit $\mathbb{G} / S$ un schéma en groupes séparé, commutatif, de dimension $g$ et soit $\operatorname{ad}_{n}: \mathbb{G}^{n} \rightarrow \mathbb{G}$ la $n$-addition. On note $\varepsilon: S \rightarrow \mathbb{G}$ la section nulle de $\mathbb{G}$. 
Proposition C.2.13. Avec les notations ci-dessus, supposons que le groupe formel $\widehat{\mathbb{G}}_{\varepsilon}$ de $\mathbb{G}$ le long de $\varepsilon$ soit isomorphe (en tant que schéma formel) au schéma formel affine

$$
\text { Specf } R\left[\left[X_{1}, \ldots, X_{g}\right]\right] \text {. }
$$

Alors l'image par $\operatorname{ad}_{n}$ du premier voisinage infinitésimal de $\mathbb{G}$ le long de $\varepsilon$

$$
\left(S_{\varepsilon}^{(1)}\right)^{\boxplus n}:=\operatorname{ad}_{n}\left(S_{\varepsilon}^{(1)} \times \cdots \times S_{\varepsilon}^{(1)}\right),
$$

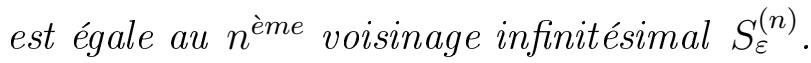

Démonstration. Notons $\widehat{\operatorname{ad}}_{n}: \widehat{\mathbb{G}}_{\varepsilon}^{n} \rightarrow \widehat{\mathbb{G}}_{\varepsilon}$ la $n$-addition formelle (déduite de $\operatorname{ad}_{n}$ par « complétion $\gg$ ), nous devons montrer que

$$
\left(\widehat{\mathbb{G}}_{\varepsilon}\right)^{(n)}=\widehat{\operatorname{ad}_{n}}\left(\left(\widehat{\mathbb{G}}_{\varepsilon}\right)^{(1)} \times \cdots \times\left(\widehat{\mathbb{G}}_{\varepsilon}\right)^{(1)}\right),
$$

égalité qui ne porte que sur le groupe formel de $\mathbb{G}$.

Lorsqu'on identifie $\widehat{\mathbb{G}}_{\varepsilon}$ à $\widehat{\mathbb{A}}^{g} \widehat{\times} S$ (hypothèse), la loi de groupe se lit sur $\widehat{\mathbb{G}}_{\varepsilon}$ via la loi de groupe formelle

$$
\mathbf{X} \oplus_{\widehat{\mathbb{G}}_{\varepsilon}} \mathbf{Y}=\left(F_{1}(\mathbf{X}, \mathbf{Y}), \ldots, F_{g}(\mathbf{X}, \mathbf{Y})\right)
$$

(où $\mathbf{X}=\left(X_{1}, \ldots, X_{g}\right)$ et $\mathbf{Y}=\left(Y_{1}, \ldots, Y_{g}\right)$ sont des $g$-uplets de variables) qui a la propriété :

$$
\mathbf{X} \oplus_{\widehat{\mathbb{G}}_{\varepsilon}} \mathbf{Y}=\mathbf{X}+\mathbf{Y}+\text { termes d'ordre } \geq 2
$$

et donc, si on note $\mathbf{Y}^{(i)}=\left(Y_{1}^{(i)}, \ldots, Y_{g}^{(i)}\right)$, on a

$$
\mathbf{Y}^{(1)} \oplus_{\widehat{\mathbb{G}}_{\varepsilon}} \cdots \oplus_{\widehat{\mathbb{G}}_{\varepsilon}} \mathbf{Y}^{(n)}=\mathbf{Y}^{(1)}+\cdots+\mathbf{Y}^{(n)}+\text { termes d'ordre } \geq 2 .
$$

En notant $\left(\mathbf{Y}^{(1)} \oplus_{\widehat{\mathbb{G}}_{\varepsilon}} \cdots \oplus_{\widehat{\mathbb{G}}_{\varepsilon}} \mathbf{Y}^{(n)}\right)_{i}$ la $i^{\text {ème }}$ composante de la somme dans $\widehat{\mathbb{G}}_{\varepsilon}$ des $\mathbf{Y}^{(j)}$, on remarque que si $\left(\alpha_{1}, \ldots, \alpha_{g}\right)$ vérifie $\alpha_{1}+\cdots+\alpha_{g} \geq n$ alors

$$
\prod_{i=1}^{g}\left(\left(\mathbf{Y}^{(1)} \oplus_{\widehat{\mathbb{G}}_{\varepsilon}} \cdots \oplus_{\widehat{\mathbb{G}}_{\varepsilon}} \mathbf{Y}^{(n)}\right)_{i}\right)^{\alpha_{i}}=\prod_{i=1}^{g}\left(Y_{i}^{(1)}+\cdots+Y_{i}^{(n)}\right)^{\alpha_{i}} \bmod \left(\mathbf{Y}^{(1)}\right)^{2}+\cdots+\left(\mathbf{Y}^{(n)}\right)^{2}
$$

Cette propriété permet de ramener la difficulté au groupe formel affine ${\widehat{\mathbb{G}_{\mathrm{a}}}}^{g}$. Par définition

$$
\left(\widehat{\mathbb{G}}^{g}\right)^{(1)}=\operatorname{Spec} \frac{R\left[\left[X_{1}, \ldots, X_{g}\right]\right]}{\left(X_{1}, \ldots, X_{g}\right)^{2}}
$$

et

$$
\left(\left({\widehat{\mathbb{G}_{\mathrm{a}}}}^{g}\right)^{(1)}\right)^{n}=\operatorname{Spec} \frac{R\left[\left[\mathbf{Y}^{(1)}, \ldots, \mathbf{Y}^{(n)}\right]\right]}{\left(\mathbf{Y}^{(1)}\right)^{2}+\cdots+\left(\mathbf{Y}^{(n)}\right)^{2}}
$$

où, comme précédemment, $\mathbf{Y}^{(i)}=\left(Y_{1}^{(i)}, \ldots, Y_{g}^{(i)}\right)$. Notons $I_{n}$ l'idéal de définition de

$$
\widehat{\operatorname{ad}_{n}}\left(\left({\widehat{\mathbb{G}_{a}}}^{g}\right)^{(1)} \times \cdots \times\left(\widehat{\mathbb{G}_{\mathrm{a}}^{g}}\right)^{(1)}\right)
$$


dans $\widehat{\mathbb{G}_{\mathrm{a}}^{g}}$. Le comorphisme de $\widehat{\operatorname{ad}_{n}}$ étant l'application

$$
\begin{aligned}
R\left[\left[X_{1}, \ldots, X_{g}\right]\right] \stackrel{{\widehat{\mathrm{ad}_{n}}}_{\longrightarrow}^{\#}}{\longrightarrow} R\left[\left[\mathbf{Y}^{(1)}, \ldots, \mathbf{Y}^{(n)}\right]\right], \\
X_{i} \stackrel{\longmapsto}{\longrightarrow} Y_{i}^{(1)}+\cdots+Y_{i}^{(n)}
\end{aligned}
$$

on a donc

$$
I_{n}=\operatorname{ker}\left(R\left[\left[X_{1}, \ldots, X_{g}\right]\right] \longrightarrow \frac{R\left[\left[\mathbf{Y}^{(1)}, \ldots, \mathbf{Y}^{(n)}\right]\right]}{\left(\mathbf{Y}^{(1)}\right)^{2}+\cdots+\left(\mathbf{Y}^{(n)}\right)^{2}}\right)
$$

et

$$
\widehat{\operatorname{ad}_{n}}\left(\left(\widehat{\mathbb{G}}_{\mathrm{a}}^{g}\right)^{(1)}\right)=\operatorname{Specf} \frac{R\left[\left[X_{1}, \ldots, X_{g}\right]\right]}{I_{n}} .
$$

Par ailleurs, par définition, on a $\left(\widehat{\mathbb{G}}_{\mathrm{a}}^{g}\right)^{(n)}=\operatorname{Specf} \frac{R\left[\left[X_{1}, \ldots, X_{g}\right]\right]}{\left(X_{1}, \ldots, X_{g}\right)^{n+1}}$. Il suffit donc de montrer que $I_{n}=I^{n+1}$ où $I=\left(X_{1}, \ldots, X_{g}\right)$.

(1) $I^{n+1} \subseteq I_{n}$

Soit $\left(\alpha_{1}, \ldots, \alpha_{g}\right) \in \mathbb{N}^{g}$ tel que $\alpha_{1}+\cdots+\alpha_{g}=n+1$. Alors

$$
\begin{aligned}
{\widehat{\operatorname{ad}_{n}}}^{\#}\left(X_{1}^{\alpha_{1}} \cdots X_{g}^{\alpha_{g}}\right) & =\left(Y_{1}^{(1)}+\cdots+Y_{1}^{(n)}\right)^{\alpha_{1}} \times \cdots \times\left(Y_{g}^{(1)}+\cdots+Y_{g}^{(n)}\right)^{\alpha_{g}} \\
& =\sum_{\sum_{j=1}^{n} \alpha_{i, j}=\alpha_{i}} c_{\underline{\alpha}} \prod_{k, \ell}\left(Y_{k}^{(\ell)}\right)^{\alpha_{k, \ell}}
\end{aligned}
$$

Or, pour tout $\underline{\alpha}=\left(\alpha_{k, \ell}\right)$ intervenant dans la somme, il existe $\ell_{0} \in\{1, \ldots, n\}$ tel que $\sum_{k=1}^{g} \alpha_{k, \ell_{0}} \geq 2$ sinon la somme totale des $\alpha_{k, \ell}$ (qui est égale à $n+1$ ) serait inférieure à $n$. Donc tous les termes de la somme sont nuls modulo l'idéal $\left(\left(\mathbf{Y}^{(1)}\right)^{2}+\cdots+\left(\mathbf{Y}^{(n)}\right)^{2}\right)$ et le monôme $X_{1}^{\alpha_{1}} \cdots X_{g}^{\alpha_{g}}$ appartient à $I_{n}$.

(2) $I_{n} \subseteq I^{n+1}$. On raisonne par récurrence sur $n$.

Pour $n=1$, c'est la définition de $I_{1}$. Supposons que $I_{k} \subseteq I^{k+1}$ pour $k \leq n-1$.

Soit $P=\sum a_{\underline{\alpha}} X_{1}^{\alpha_{1}} \cdots X_{g}^{\alpha_{g}} \in I_{n}$. Par définition, il existe des polynômes $U_{i, j, k}$ tels que

$$
P\left(Y_{1}^{(1)}+\cdots+Y_{1}^{(n)}, \ldots, Y_{g}^{(1)}+\cdots+Y_{g}^{(n)}\right)=\sum_{\substack{1 \leq k \leq n \\ 1 \leq i, j \leq g}} Y_{i}^{(k)} Y_{j}^{(k)} U_{i, j, k} .
$$

En prenant, pour tout $i, Y_{i}^{(n)}=0$, l'égalité ci-dessus signifie que $P \in I_{n-1}$, donc, par hypothèse de récurrence, $P$ appartient à $I^{n}$, ce qui implique que $a_{\underline{\alpha}}=0$ pour $|\underline{\alpha}| \leq n-1$. Comme les monômes $X^{\underline{\alpha}}$ appartiennent à $I^{n+1}$ pour $|\underline{\alpha}| \geq n+1$, il suffit de montrer que $a_{\underline{\alpha}}=0$ pour $|\underline{\alpha}|=n$. L'égalité (C.4) devient (modulo l'idéal $\left.\left(\mathbf{Y}^{(1)}\right)^{2}+\cdots+\left(\mathbf{Y}^{(n)}\right)^{2}\right)$ :

$$
\sum_{|\underline{\alpha}|=n} a_{\underline{\alpha}}\left(Y_{1}^{(1)}+\cdots+Y_{1}^{(n)}\right)^{\alpha_{1}} \cdots\left(Y_{g}^{(1)}+\cdots+Y_{g}^{(n)}\right)^{\alpha_{g}}=0 .
$$

On développe cette expression avec la formule du multinôme :

$$
\sum_{|\underline{\alpha}|=n} a_{\underline{\alpha}} \sum_{\alpha_{k, 1}+\cdots+\alpha_{k, n}=\alpha_{k}}\left(\prod_{m=1}^{g} \frac{\alpha_{m} !}{\alpha_{m, 1} ! \cdots \alpha_{m, n} !}\right)\left(\prod_{k, \ell}\left(Y_{k}^{(\ell)}\right)^{\alpha_{k, \ell}}\right)=0,
$$


et dès qu'il existe $\ell_{0} \in\{1, \ldots, n\}$ tel que $\sum_{k=1}^{g} \alpha_{k, \ell_{0}} \geq 2$, le terme monomial de la seconde somme disparaît. Il ne reste donc que les termes pour lesquels $\sum_{k=1}^{g} \alpha_{k, \ell} \leq 1$ (pour tout $\ell$ ) et comme la somme totale des $\alpha_{k, \ell}$ vaut $n$, nécessairement on a $\sum_{k=1}^{g} \alpha_{k, \ell}=1$. L'équation (C.5) devient

$$
\sum_{|\underline{\alpha}|=n} a_{\underline{\alpha}}\left\{\prod_{k=1}^{g} \alpha_{k} ! \times \sum_{\substack{\alpha_{k, 1}+\cdots+\alpha_{k, n}=\alpha_{k} \\ \sum_{h=1}^{g} \alpha_{h, \ell}=1}} \prod_{k, \ell}\left(Y_{k}^{(\ell)}\right)^{\alpha_{k, \ell}}\right\}=0 .
$$

Soient $\underline{\alpha} \in \mathbb{N}^{g}$ et un $(n \times g)$-uplet d'entiers positifs $\left(\alpha_{k, \ell}\right)_{\substack{1 \leq \ell \leq n \\ 1 \leq k \leq g}}$ vérifiant les conditions

$$
\sum_{\ell=1}^{n} \alpha_{k, l}=\alpha_{k} \quad \text { et } \quad \sum_{k=1}^{g} \alpha_{k, \ell}=1 .
$$

On note $\sigma_{\underline{\alpha}}$ l'application de $\{1, \ldots, n\}$ dans $\{1, \ldots, g\}$ qui à $\ell$ associe l'unique entier $k$ tel que $\alpha_{k, \ell}=1$ et $\alpha_{m, \ell}=0$ pour $m \neq k$. L'équation (C.6) s'écrit alors :

$$
\sum_{\underline{\alpha}}\left\{a_{\underline{\alpha}}\left(\prod_{k=1}^{g} \alpha_{k} !\right)\left(\sum_{\sigma_{\underline{\alpha}}} \prod_{\ell=1}^{n} Y_{\sigma_{\underline{\alpha}}(\ell)}^{(\ell)}\right)\right\}=0 .
$$

On dérive (C.7) successivement par rapport aux variables $Y_{\sigma(1)}^{(1)}, \ldots, Y_{\sigma(n)}^{(n)}$. Dans le membre de gauche de (C.7), on ne conserve que les $\alpha_{j, \ell}$ tel que pour tout $\ell: \alpha_{\sigma(\ell), \ell}=1$. Il ne reste donc que $a_{\underline{\alpha}} \times \prod_{k=1}^{g} \alpha_{k}$ ! (constante). Le membre de droite est, quant à lui, nul modulo $\left(\mathbf{Y}^{(1)}\right)+$ $\cdots+\left(\mathbf{Y}^{(n)}\right)$. Donc finalement $a_{\underline{\alpha}}=0$ car $R$ est une $\mathbb{Q}$-algèbre.

Remarque : Cette proposition est une reformulation géométrique de l'identité, valable pour une fonction $f$ d'une variable, analytique au voisinage de 0 :

$$
\frac{\partial}{\partial z_{1}} \cdots \frac{\partial}{\partial z_{n}} f\left(z_{1}+\cdots+z_{n}\right)_{\mid z_{i}=0}=\left(\left(\frac{\partial}{\partial z}\right)^{n} f\right)(0)
$$

Le lemme clef au coeur de la proposition C.2.13 s'énonce ainsi :

Lemme. Soient $(n, g) \in(\mathbb{N} \backslash\{0\})^{2}$ et $R$ une $\mathbb{Q}$-algèbre commmutative.

Si $f \in R\left[\left[X_{1}, \ldots, X_{g}\right]\right]$ alors les assertions suivantes sont équivalentes :

1) $f\left(X_{1}, \ldots, X_{g}\right) \in\left(X_{1}, \ldots, X_{g}\right)^{n+1}$

2) $f\left(X_{1}^{(1)}+\cdots+X_{1}^{(n)}, \ldots, X_{g}^{(1)}+\cdots+X_{g}^{(n)}\right) \in\left(X_{1}^{(1)}, \ldots, X_{g}^{(1)}\right)^{2}+\cdots+\left(X_{1}^{(n)}, \ldots, X_{g}^{(n)}\right)^{2}$

Ce lemme permet de montrer plus généralement que $\left(S_{\mathcal{W}}^{(1)}\right)^{\boxplus n}=S_{\mathcal{W}}^{(n)}$, dans le cas où $S=$ $\operatorname{Spec} R, R$ étant un anneau pouvant être plongé dans $\mathbb{C}$, et $\mathcal{W}$ un sous-espace de $t_{\mathbb{G} / S}$, avec $\mathbb{G} \rightarrow S$ lisse. En effet, comme $S_{\mathcal{W}}^{(n)}$ est l'adhérence schématique dans $\mathbb{G}$ de $S_{\mathcal{W} \otimes \text { Frac } R}^{(n)}$ et même de $S_{\mathcal{W} \otimes \mathbb{C}}^{(n)}$, il suffit de démontrer que $\left(S_{\mathcal{W} \otimes \mathbb{C}}^{(1)}\right)^{\boxplus n}$ et $S_{\mathcal{W} \otimes \mathbb{C}}^{(n)}$ sont isomorphes. Or, comme nous l'avons vu au début de ce paragraphe, en notant $\left(w_{1}, \ldots, w_{h}\right)$ une base de $\mathcal{W} \otimes \mathbb{C}$, l'anneau des fonctions du schéma (affine) $S_{\mathcal{W} \otimes \mathbb{C}}^{(n)}$ est défini par la condition $f\left(\mathbf{u}+z_{1} w_{1}+\cdots+z_{h} w_{h}\right) \in$ $\left(z_{1}, \ldots, z_{h}\right)^{n+1}$ (où u est un logarithme de $\varepsilon(\mathbb{C}) \in \mathbb{G}(\mathbb{C})$ ). L'anneau des fonctions de $\left(S_{\mathcal{W}}^{(1)}\right)^{\boxplus n}=$ $S_{\mathcal{W}}^{(n)}$, quant à lui, est défini par l'appartenance de

$$
f\left(\mathbf{u}+\left(z_{1}^{(1)} w_{1}+\cdots+z_{h}^{(1)} w_{h}\right)+\cdots+\left(z_{1}^{(n)} w_{1}+\cdots+z_{h}^{(n)} w_{h}\right)\right)
$$


à l'idéal

$$
\left(z_{1}^{(1)}, \ldots, z_{h}^{(1)}\right)^{2}+\cdots+\left(z_{1}^{(n)}, \ldots, z_{h}^{(n)}\right)^{2} .
$$

Le lemme clef permet d'identifier ces deux anneaux, donnant du même coup, l'isomorphisme de schémas voulu.

L'hypothèse de la proposition C.2.13 sera vérifiée, par exemple, lorsque $R$ est principal et $\mathbb{G} / S$ est lisse ( $c f$. la proposition C.3.11).

\section{$\S$ C.3. Notion de lissité}

La notion de lissité, riche et multiforme, occupe une place centrale en géométrie algébrique. De nombreuses références permettent de s'initier à ses différentes facettes. Pour le point de vue 《 relèvement formel d'une section », on pourra consulter les premières pages de l'exposé de L. Illusie dans [4] puis, pour approfondir, [43] (quatrième partie). Si on s'intéresse aux schémas en groupes lisses, [14] et [42] sont les plus complets. On donne dans la bibliographie d'autres références plus éparses. Cependant pour la commodité du lecteur, nous rappelons quelques définitions, extraites de ces livres.

Définition C.3.1. Le $S$-schéma $X \rightarrow S$ est différentiellement lisse si et seulement si

i) $\Omega_{X / S}^{1}$ est un $\mathcal{O}_{X}$-module localement projectif.

ii) L'homomorphisme canonique $\mathbf{S}\left(\Omega_{X / S}^{1}\right) \rightarrow \mathcal{G} r\left(\mathcal{P}_{X / S}\right)$ est un isomorphisme d'algèbres graduées.

Définition C.3.2. Un $S$-schéma $X \rightarrow S$ est formellement lisse (resp. formellement étale) si pour tout schéma affine $Z$ au-dessus de $S$ et tout sous-schéma fermé $Z_{0}$ de $Z$ défini par un idéal de carré nul*, l'application $X(Z) \rightarrow X\left(Z_{0}\right)$ est surjective (resp. bijective).

On rappelle qu'un morphisme de schémas $\pi: X \rightarrow S$ est localement de type fini si, $\forall x \in X$, il existe un voisinage ouvert affine Spec $R$ de $\pi(x)$ et un voisinage ouvert affine $\operatorname{Spec} B$ de $x$ tel que $\pi(\operatorname{Spec} B) \subseteq$ Spec $R$ et que $B$ soit isomorphe à un quotient de la forme $R\left[X_{1}, \ldots, X_{g}\right] / I$, où $I$ est un idéal de $R\left[X_{1}, \ldots, X_{g}\right]$.

Un morphisme $\pi: X \rightarrow S$ est localement de présentation finie si l'idéal $I$ qui précède est de type fini.

Définition C.3.3. Le $S$-schéma $X \rightarrow S$ est lisse (resp. étale) s'il est localement de présentation finie et formellement lisse (resp. formellement étale).

Remarque C.3.4. Il est important de préciser le schéma de base au-dessus duquel le schéma est lisse. Si la lissité se conserve par extension de la base, elle ne se conserve sûrement pas par diminution de la base. Par exemple une courbe définie sur un anneau de Dedekind $R$, lisse sur le corps des fractions de $R$, ne l'est pas toujours sur $R$. De même, un célèbre théorème de J.-M. Fontaine [36] affirme qu'il n'existe aucune variété abélienne (de dimension relative $\geq 1$ ) lisse sur $\mathbb{Z}$ (ce qui impliquerait qu'elle a bonne réduction en tous les idéaux premiers de $\mathbb{Z})$.

Remarque C.3.5. La lissité est une notion locale. Le schéma $X / S$ est lisse si et seulement si $\forall x \in X$ il existe un ouvert $U_{x}$ contenant $x$ tel que $U_{x} \rightarrow S$ soit lisse.

*I.e. un épaississement d'ordre 1. 
Rappelons le critère jacobien de lissité :

Théorème C.3.6. Soient $X / S$ un schéma localement de présentation finie, $x \in X$ et $s$ son image dans $S$. Alors $X / S$ est lisse au point $x$ si et seulement s'il existe des voisinages ouvert affines $\operatorname{Spec}\left(\frac{R\left[X_{1}, \ldots, X_{\ell+r}\right]}{\left(f_{1}, \ldots, f_{\ell}\right)}\right)$ et $\operatorname{Spec} R$ de $x$ et s respectivement tels que les mineurs d'ordre $\ell$ de la matrice jacobienne $\left(\frac{\partial f_{i}}{\partial X_{j}}\right)$ engendrent $\frac{R\left[X_{1}, \ldots, X_{\ell+r}\right]}{\left(f_{1}, \ldots, f_{\ell}\right)}$ (en tant qu'idéal).

Il est parfois judicieux, si on veut démontrer la lissité d'un morphisme (en ramenant la base au spectre d'un corps), d'utiliser au préalable la

Proposition C.3.7 (Proposition 8 du chapitre 2 de [14]). Soient $\pi: X \rightarrow S$ un morphisme localement de présentation finie, $x$ un point de $X$ et $s=\pi(x)$ son image dans $S$ via $\pi$. Alors $\pi$ est lisse au point $x$ si et seulement si $\pi$ est plat en $x$ et la fibre $X_{s}=X \times{ }_{S} \operatorname{Spec} k(s) \rightarrow$ Spec $k(s)$ est lisse au point $x$.

Cette proposition permet de se ramener à une base qui est un corps (de caractéristique quelconque).

Proposition C.3.8. Si X/S est lisse alors il est différentiellement lisse.

Proposition C.3.9 (Critère pour les schémas en groupes). Le schéma en groupes $\mathbb{G} / S$ est différentiellement lisse si et seulement s'il est lisse aux points de $\varepsilon(S)$, où $\varepsilon: S \rightarrow \mathbb{G}$ est la section nulle de $\mathbb{G}$.

La notion de lissité peut s'exprimer à l'aide du foncteur représentable $\mathbf{F}_{X}:$ Sch $/ S \rightarrow$ Ens, de la catégorie des schémas au-dessus de $S$ dans la catégorie des ensembles, qui à $Z$ associe $X(Z)$. Ainsi, le schéma $X / S$ est lisse si et seulement si

i) $\mathbf{F}_{X}$ commute aux limites projectives de schémas affines.

ii) Pour tout schéma affine $Z$ au-dessus de $S$, pour tout épaississement $Z_{0}$ d'ordre 1 de $Z$, l'application $\mathbf{F}_{X}(Z) \rightarrow \mathbf{F}_{X}\left(Z_{0}\right)$ est surjective.

Ce point de vue ouvre la porte à d'éventuelles généralisations de la notion de lissité pour des objets d'une certaine catégorie munie d'un foncteur contravariant à valeurs dans la catégories des ensembles. Par exemple, pour les schémas formels, on a :

Définition C.3.10. On dit qu'un schéma formel $\mathfrak{X}$ sur $S$ est lisse s'il est isomorphe au complété d'un fibré vectoriel $X=\mathbb{V}(\mathcal{E})$ (le long de sa section canonique ( $c f$. $\S$ C.2.b), où $\mathcal{E}$ est un $\mathcal{O}_{S}$-module localement libre.

En fait on a la proposition suivante (corollaire 16.9 .9 de [43]) :

Proposition C.3.11. Soient $X / S$ un schéma localement nothérien et $\varepsilon: S \rightarrow X$ une $S$ section de $X$. Rappelons ${ }^{*}$ que $\mathcal{O}_{S_{\varepsilon}^{(\infty)}}$ désigne la limite projective des $\mathcal{O}_{S_{\varepsilon}^{(n)}}$ (cf. paragraphe C.2.a). Alors $X / S$ est lisse si et seulement si, pour tout $s \in S$, il existe un voisinage ouvert affine $U=\operatorname{Spec} R$ de s tel que l'on ait un isomorphisme (topologique) de $\mathcal{O}_{U}$-algèbre :

$$
\mathcal{O}_{S_{\varepsilon}^{(\infty)} / U} \simeq \mathcal{O}_{U}\left[\left[X_{1}, \ldots, X_{g}\right]\right]
$$

De plus g est la dimension relative en $\varepsilon(s)$ de $X / S$, ou, ce qui revient au même ici, le rang de l'espace tangent $t_{X / S, s}$.

\footnotetext{
${ }^{*}$ Si $X / S$ est nœthérien et séparé, $\mathcal{O}_{S_{\varepsilon}^{(\infty)}} \simeq \widehat{\mathcal{O}}_{X}(c f . \S$ C.2.a $)$.
} 
Ainsi les propriétés différentielles d'un schéma lisse sont les mêmes que celles d'un anneau de polynômes. Dans le cas où $S=\operatorname{Spec} R$ où $R$ est un anneau de Dedekind principal, on pourra consulter le second chapitre de la thèse de P. GraftiEAux [38] pour une version globale de cette proposition.

\section{$\S$ C.4. Exemples de morphismes lisses}

\section{$\S$ C.4.a. Fibrés vectoriels et fibrés projectifs}

Les résultats que nous présentons dans ce paragraphe sont relativement généraux.

Proposition C.4.1. Soit $\mathcal{E}$ un $\mathcal{O}_{S}$-module localement libre de type fini. Le fibré vectoriel $\mathbb{V}(\mathcal{E})$ et le fibré projectif $\mathbb{P}(\mathcal{E})$ sont des schémas lisses sur $S$.

En particulier si $R$ est un anneau (commutatif, unitaire) et $n$ un entier naturel non nul, le schéma $\mathbb{P}_{R}^{n}$ est lisse sur $R$.

Lorsque $E$ est une courbe elliptique définie sur un corps $K$, corps des fractions d'un anneau de Dedekind $R$, d'équation de Weierstrass

$$
y^{2}+a_{1} x y+a_{3} y=x^{3}+a_{2} x^{2}+a_{4} x+a_{6},
$$

où les coefficients $a_{i}$ appartiennent à $R$, alors le sous-schéma fermé $\mathcal{E}$ de $\mathbb{P}_{R}^{2}$ défini par cette équation (homogénéisée) est lisse sur $R$ si et seulement si $E$ a bonne réduction en tous les premiers de $R$, i.e. si et seulement si le discriminant

$$
\begin{aligned}
\Delta_{\mathcal{E}}:= & -\left(a_{1}^{2}+4 a_{2}\right)^{2}\left(a_{1}^{2} a_{6}+4 a_{2} a_{6}-a_{1} a_{3} a_{4}+a_{2} a_{3}^{2}-a_{4}^{2}\right) \\
& -8\left(2 a_{4}+a_{1} a_{3}\right)^{3}-27\left(a_{3}^{2}+4 a_{6}\right)^{2} \\
& +9\left(a_{1}^{2}+4 a_{2}\right)\left(2 a_{4}+a_{1} a_{3}\right)\left(a_{3}^{2}+4 a_{6}\right)
\end{aligned}
$$

de l'équation de Weierstrass définissant $\mathcal{E}$ est inversible dans $R$. Dans ce cas, on montre même que le schéma $\mathcal{E} \rightarrow$ Spec $R$ est un modèle de Néron* de $E$ ( $c f$. corollaire 6.3 du chapitre IV de [75], page 329).

\section{$\S$ C.4.b. Schémas en groupes sur un corps}

Théorème C.4.2 (CARTIER). Tout schéma en groupes $\mathbb{G}$ localement de présentation finie au-dessus d'un corps $k$ de caractéristique nulle est lisse.

Esquisse de démonstration. Le $k$-espace vectoriel $\omega_{\mathbb{G} / S}^{1}$ est libre de dimension finie donc $\mathbb{G}$ est différentiellement lisse car la caractéristique de $k$ est nulle ; donc $\mathbb{G}$ est lisse en au moins un point de $k$, donc $\mathbb{G}$ est lisse sur $k$ d'après la proposition C.3.9. Il y a plus de détails dans [42], page 327 .

Malheureusement, en arithmétique, on considère le plus souvent un schéma en groupes au-dessus de l'anneau des entiers d'un corps de nombres ou d'un corps fini, schéma qui n'est pas lisse en général. On aura donc à rajouter cette hypothèse si besoin est.

*I.e. qu'à la propriété de lissité s'ajoute une structure de schéma en groupes et une propriété d'existence et d'unicité d'un relèvement de morphismes lisses sur la fibre générique ( Néron mapping property). Pour une définition précise, $c f .[14]$. 


\section{$\S$ C.4.c. Lissité des sous-groupes de $\mathbb{G}_{\mathrm{a}}^{g}$ et un calcul de $\Omega^{1}$.}

Soit $S$ un schéma. Un sous-schéma en groupes $\mathbb{G}^{\prime}$ de $\mathbb{G}_{a}^{g} / \mathbb{Z}$ s'écrit $a_{i, 1} X_{1}+\cdots+a_{i, g} X_{g}=0$ $(1 \leq i \leq r)$. Le groupe $\mathbb{G}^{\prime} \times_{\mathbb{Z}} S$ est lisse sur $S$ si les facteurs invariants de la matrice $\left(a_{i, j}\right)$ sont inversibles dans $S$ (i.e. dans $k(s)$ pour tout $s \in S$ ). Notons $\varepsilon$ la section nulle de $\mathbb{G}_{a}^{g} / \mathbb{Z}$. Si $n>0$, le $n^{\text {ème }}$ voisinage infinitésimal $\left(\mathbb{G}_{a}^{g}\right)^{(n)}$ n'est pas lisse sur $\mathbb{Z}$ (car non réduit, voir proposition 17.5.7 de [43]). Ainsi le $\mathbb{Z}$-module des différentielles $\Omega_{\left(\mathbb{G}_{a}^{g}\right)^{(n)} / \mathbb{Z}}^{1}$ comporte une partie de torsion. À titre d'exemple, nous allons calculer explicitement ce module.

Proposition C.4.3. Avec les notations ci-dessus, notons $\mu$ la fonction de Möbius habituelle. Alors $\Omega_{\left(\mathbb{G}_{a}^{g}\right)^{(n)} / \mathbb{Z}}^{1}$ est isomorphe en tant que $\mathbb{Z}$-module*

$$
\mathbb{Z}^{n\left(\begin{array}{c}
n+g \\
n+1
\end{array}\right)} \bigoplus_{d \mid n+1}^{\oplus}(\mathbb{Z} / d \mathbb{Z})^{\sum_{j \mid \frac{n+1}{d}} \mu(j)\left(\begin{array}{c}
g+\frac{n+1}{d d}-1 \\
g-1
\end{array}\right)}
$$

Démonstration. La preuve utilise le lemme classique suivant [48] :

Lemme C.4.4. Soient $X:=\operatorname{Spec} B$ où $B=\frac{R\left[X_{\lambda}\right]_{\lambda \in \Lambda}}{J},\left(X_{\lambda}\right)_{\lambda \in \Lambda}$ est une famille d'indéterminées et $J$ un idéal de $R\left[X_{\lambda}\right]_{\lambda \in \Lambda}$. Alors on a

$$
\Omega_{X / R}^{1}=\frac{\oplus_{\lambda \in \Lambda} B \cdot \mathrm{d} X_{\lambda}}{<B . \mathrm{d} f>_{f \in J}}
$$

où $<B . \mathrm{d} f>_{f \in J}$ désigne l'idéal de $B$ engendré par les différentielles $\mathrm{d} f$.

Ici $X=\left(\mathbb{G}_{\mathrm{a}}^{g}\right)^{(n)}=\operatorname{Spec} B$ où $B=\frac{\mathbb{Z}\left[X_{1}, \ldots, X_{g}\right]}{\left(X_{1}, \ldots, X_{g}\right)^{n+1}}$ et on a

$$
\Omega_{\left(\mathbb{G}_{a}^{g}\right)^{(n)} / \mathbb{Z}}^{1}=\frac{B \cdot \mathrm{d} X_{1} \oplus \cdots \oplus B \cdot \mathrm{d} X_{g}}{\left(\sum_{h=1}^{g} \alpha_{h} X^{\underline{\alpha}} X_{h}^{-1} \mathrm{~d} X_{h}\right)_{|\underline{\alpha}|=n+1}} .
$$

En utilisant la notation habituelle $X^{\underline{\alpha}}=X_{1}^{\alpha_{1}} \cdots X_{g}^{\alpha_{g}}$, un élément quelconque de $B \cdot d X_{1} \oplus \cdots \oplus B . \mathrm{d} X_{g}$ s'écrit

$$
\underbrace{\sum_{h=1}^{g} \sum_{|\underline{\alpha}|<n} a_{\underline{\alpha}}^{(h)} X^{\underline{\alpha}} \mathrm{d} X_{h}}_{\text {pas modifié par le quotient }}+\sum_{h=1}^{k} \sum_{|\underline{\alpha}|=n} a_{\underline{\alpha}}^{(h)} X^{\underline{\alpha}} \mathrm{d} X_{h} .
$$

La première partie est donc libre de rang $g\left(\begin{array}{c}n-1+g \\ g\end{array}\right)$. Quant à l'autre somme, cela revient à comprendre, si $A_{n}$ désigne la somme directe

$$
\underset{\substack{|\ell|=n \\ i \in\{1, \ldots, g\}}}{\bigoplus} \mathbb{Z} \cdot X^{\underline{\ell}} \mathrm{d} X_{i}
$$

la structure de $\mathbb{Z}$-module du quotient $\frac{A_{n}}{\oplus_{|\underline{\ell}|=n+1} \mathbb{Z} . \mathrm{d} X \underline{\underline{\ell}}}$. Pour cela, considérons $\underline{\ell} \in \mathbb{N}^{g}$ tel que $|\underline{\ell}|=n+1$ et ayant exactement $m$ composantes non nulles $\ell_{i_{1}}, \ldots, \ell_{i_{m}}$. Notons $B_{n}^{(\underline{\ell})}$ le sous-module :

$$
\mathbb{Z} . X^{\ell} X_{i_{1}}^{-1} \mathrm{~d} X_{i_{1}} \oplus \cdots \oplus \mathbb{Z} . X^{\ell} X_{i_{m}}^{-1} \mathrm{~d} X_{i_{m}}
$$

*Et aussi en tant que $\mathbb{Z}$-algèbre. 
Alors $A_{n}=\oplus_{\underline{\ell}} B_{n}^{(\underline{\ell})}$ (cela découle directement des définitions) et, plus intéressant, la situation reste scindée en quotientant :

$$
\frac{A_{n}}{\oplus_{|\underline{\ell}|=n+1} \mathbb{Z} \cdot \mathrm{d}\left(X^{\underline{\ell}}\right)} \simeq \oplus_{\underline{\ell}}\left(\frac{B_{n}^{(\underline{\ell})}}{\mathbb{Z} \cdot \mathrm{d} X^{\underline{\ell}}}\right)
$$

Par définition de $B_{n}^{(\ell)}$ il est clair que $B_{n}^{(\ell)} / \mathbb{Z} \cdot \mathrm{d} X^{\underline{\ell}} \simeq \mathbb{Z}^{m} / \mathbb{Z} \cdot \underline{\ell}^{\prime}$ où $\underline{\ell}^{\prime}=\left(\ell_{i_{1}}, \ldots, \ell_{i_{m}}\right)$.

Lemme C.4.5. Soit $\underline{\ell}=\left(\ell_{1}, \ldots, \ell_{m}\right)$ un m-uplet dont aucune composante n'est nulle et notons d le pgcd des $\ell_{i}$. Alors il existe un isomorphisme de $\mathbb{Z}$-modules :

$$
\mathbb{Z}^{m} / \mathbb{Z} \cdot \underline{\ell} \simeq \mathbb{Z}^{m-1} \oplus \mathbb{Z} / d \mathbb{Z}
$$

Démonstration. D'après le théorème de structure des $\mathbb{Z}$-modules de type fini, le groupe $\mathbb{Z}^{m} / \mathbb{Z} . \underline{\ell}$ est somme directe d'une puissance de $\mathbb{Z}$ et d'un groupe de torsion. En tensorisant par $\mathbb{Q}$, il est immédiat que la partie libre est bien $\mathbb{Z}^{m-1}$. Pour la partie de torsion, considérons $a \in \mathbb{Z}^{m}$ qui est de $k$-torsion exactement. Il existe donc $\mu \in \mathbb{Z}$ tel que, pour tout $i \in\{1, \ldots, m\}, k a_{i}=\mu h_{i}$. Soient $b_{1}, \ldots, b_{n} \in \mathbb{Z}$ tel que $\sum_{i=1}^{m} b_{i} \ell_{i}=d$ (Bézout). Alors $k\left(\sum b_{i} a_{i}\right)=\mu d$, et comme $k$ est premier avec $\mu$ (car $a$ est de $k$ torsion exactement), $k$ divise $d$. Réciproquement si un entier $k$ divise $d$, il existe $a=\left(\ell_{1} / d, \ldots, \ell_{m} / d\right)$ qui est de $k$-torsion exactement. Donc le groupe de torsion est cyclique, engendré par $\frac{1}{d} \underline{\ell}$.

Comme il y a $\left(\begin{array}{c}g \\ m\end{array}\right)$ façons de choisir $\underline{\ell} \in \mathbb{N}^{g}$ ayant exactement $m$ composantes non nulles, de la décomposition (C.10) on déduit

$$
\Omega_{\left(\mathbb{G}_{a}^{g}\right)^{(n)} / \mathbb{Z}}^{1} \simeq \mathbb{Z}^{g\left(\begin{array}{c}
n-1+g \\
g
\end{array}\right)} \bigoplus \underset{\substack{\ell^{\prime}=\left(\ell_{1}, \ldots, \ell_{m}\right) \\
\left|\underline{\ell}^{\prime}\right|=n+1, \ell_{1}, \ldots, \ell_{m} \geq 1}}{\oplus}\left(\mathbb{Z}^{m} / \mathbb{Z}^{\prime} \cdot \underline{\prime}^{\prime}\right)^{\left(\begin{array}{l}
g \\
m
\end{array}\right)}
$$

D'après le lemme C.4.5, la partie libre de ce module est de rang

$$
g\left(\begin{array}{c}
n-1+g \\
g
\end{array}\right)+\sum_{m=1}^{g}(m-1)\left(\begin{array}{c}
g \\
m
\end{array}\right)\left(\begin{array}{c}
n \\
m-1
\end{array}\right)
$$

car le cardinal de l'ensemble $\left\{\left(\ell_{1}, \ldots, \ell_{m}\right) \in(\mathbb{N} \backslash\{0\})^{m} ; \ell_{1}+\cdots+\ell_{m}=n+1\right\}$ est $\left(\begin{array}{c}n \\ m-1\end{array}\right)$.

Lemme C.4.6. Les égalités suivantes sont vérifiées pour $a, b$ entiers naturels :

1) $\sum_{k=0}^{a}\left(\begin{array}{l}a \\ k\end{array}\right)\left(\begin{array}{l}b \\ k\end{array}\right)=\left(\begin{array}{c}a+b \\ a\end{array}\right)$.

2) $\sum_{k=0}^{a} k\left(\begin{array}{l}a \\ k\end{array}\right)\left(\begin{array}{l}b \\ k\end{array}\right)=a\left(\begin{array}{c}a+b-1 \\ a\end{array}\right)$.

\section{Démonstration.}

1) On a $\left(\sum_{k=0}^{a}\left(\begin{array}{l}a \\ k\end{array}\right) X^{k}\right)\left(\sum_{k=0}^{b}\left(\begin{array}{l}b \\ k\end{array}\right) X^{k}\right)=(1+X)^{a+b}$. En comparant les coefficients devant $X^{a}$ on obtient la première égalité.

2) On a $\sum_{k=0}^{a} k\left(\begin{array}{l}a \\ k\end{array}\right) X^{k}=X \frac{\mathrm{d}}{\mathrm{d} X}\left(\sum_{k=0}^{a}\left(\begin{array}{l}a \\ k\end{array}\right) X^{k}\right)=a X(1+X)^{a-1}$. Donc en multipliant des deux côtés par $(1+X)^{b}$, on obtient $\sum_{k, j} k\left(\begin{array}{l}a \\ k\end{array}\right)\left(\begin{array}{l}b \\ j\end{array}\right) X^{k+j}=a X(1+X)^{a+b-1}$. En comparant les coefficients devant $X^{a}$, on obtient la seconde égalité. 
L'utilisation du lemme C.4.6 avec $a=g-1$ et $b=n$, puis $a=g$ et $b=n+1$ donne

$$
\begin{aligned}
& \sum_{m=1}^{g}(m-1)\left(\begin{array}{c}
g \\
m
\end{array}\right)\left(\begin{array}{c}
n \\
m-1
\end{array}\right)=\sum_{m=1}^{g-1} m\left(\begin{array}{c}
g \\
m+1
\end{array}\right)\left(\begin{array}{c}
n \\
m
\end{array}\right) \\
& =\sum_{m=1}^{g-1}(m+1)\left(\begin{array}{c}
g \\
m+1
\end{array}\right)\left(\begin{array}{c}
n \\
m
\end{array}\right)-\sum_{m=1}^{g-1}\left(\begin{array}{c}
g \\
m+1
\end{array}\right)\left(\begin{array}{c}
n \\
m
\end{array}\right) \\
& =g \sum_{m=1}^{g-1}\left(\begin{array}{c}
g-1 \\
m
\end{array}\right)\left(\begin{array}{c}
n \\
m
\end{array}\right)-\frac{1}{n+1} \sum_{m=1}^{g-1}\left(\begin{array}{c}
g \\
m+1
\end{array}\right)\left(\begin{array}{c}
n+1 \\
m+1
\end{array}\right)(m+1) \\
& =g \sum_{m=1}^{g-1}\left(\begin{array}{c}
g-1 \\
m
\end{array}\right)\left(\begin{array}{c}
n \\
m
\end{array}\right)-\frac{1}{n+1} \sum_{m=2}^{g}\left(\begin{array}{c}
g \\
m
\end{array}\right)\left(\begin{array}{c}
n+1 \\
m
\end{array}\right) m \\
& =g\left(\left(\begin{array}{c}
n+g-1 \\
n
\end{array}\right)-1\right)-\frac{1}{n+1}\left((n+1)\left(\begin{array}{l}
g+n \\
n+1
\end{array}\right)-g(n+1)\right) \\
& =g\left(\begin{array}{c}
n+g-1 \\
n
\end{array}\right)-\left(\begin{array}{c}
g+n \\
n+1
\end{array}\right)
\end{aligned}
$$

La partie libre de $\Omega_{\left(\mathbb{G}_{\mathrm{a}}^{g}\right)^{(n)} / \mathbb{Z}}^{1}$ est donc bien de rang $g\left(\begin{array}{c}n+g-1 \\ g\end{array}\right)+g\left(\begin{array}{c}n+g-1 \\ g-1\end{array}\right)-\left(\begin{array}{c}n+g \\ g-1\end{array}\right)=n\left(\begin{array}{c}n+g \\ n+1\end{array}\right)$.

Pour expliciter les exposants de la partie de torsion, nous aurons besoin d'un :

Lemme C.4.7. Soit $n \in \mathbb{N} \backslash\{0\}$ et $d$ un diviseur de $n$. On note $\delta_{n, d}^{(k)}$ le cardinal de l'ensemble

$$
\left\{\left(\alpha_{1}, \ldots, \alpha_{k}\right) \in(\mathbb{N} \backslash\{0\})^{k} ; \alpha_{1}+\cdots+\alpha_{k}=n, \operatorname{pgcd}\left(\alpha_{1}, \ldots, \alpha_{k}\right)=d\right\}
$$

et $\delta_{n}^{(k)}:=\delta_{n, 1}^{(k)}$.

Alors

$$
\delta_{n, d}^{(k)}=\delta_{n / d}^{(k)}
$$

et

$$
\delta_{n}^{(k)}=\sum_{x \mid n} \mu(x)\left(\begin{array}{c}
n / x-1 \\
k-1
\end{array}\right) .
$$

Démonstration. La première égalité est claire.

Pour la seconde, on remarque que $\sum_{d \mid n} \delta_{n, d}^{(k)}=\left|\left\{\left(\alpha_{1}, \ldots, \alpha_{k}\right) \in(\mathbb{N} \backslash\{0\})^{k} ; \alpha_{1}+\ldots+\alpha_{k}=n\right\}\right|$ donc

$$
\sum_{d \mid n} \delta_{d}^{(k)}=\left(\begin{array}{l}
n-1 \\
k-1
\end{array}\right) .
$$

La formule d'inversion de Möbius donne alors le résultat.

D'après les lemmes C.4.5 et C.4.7, l'exposant de $\mathbb{Z} / d \mathbb{Z}$ est :

$$
\begin{aligned}
\sum_{m=1}^{g}\left(\begin{array}{c}
g \\
m
\end{array}\right) \delta_{n+1, d}^{(m)} & =\sum_{m=1}^{g}\left(\begin{array}{c}
g \\
m
\end{array}\right) \sum_{x \mid \frac{n+1}{d}} \mu(x)\left(\begin{array}{c}
\frac{n+1}{d x}-1 \\
m-1
\end{array}\right) \\
& =\sum_{x \mid \frac{n+1}{d}} \mu(x) \sum_{m=1}^{g}\left(\begin{array}{c}
g \\
m
\end{array}\right)\left(\begin{array}{c}
\frac{n+1}{d x}-1 \\
m-1
\end{array}\right)
\end{aligned}
$$


Or

$$
\begin{aligned}
\sum_{m=1}^{g}\left(\begin{array}{c}
g \\
m
\end{array}\right)\left(\begin{array}{c}
\frac{n+1}{d x}-1 \\
m-1
\end{array}\right) & =\frac{d x}{n+1} \sum_{m=1}^{g}\left(\begin{array}{c}
g \\
m
\end{array}\right)\left(\begin{array}{c}
\frac{n+1}{d x} \\
m
\end{array}\right) m \\
& =\left(\begin{array}{c}
g+\frac{n+1}{d x}-1 \\
g-1
\end{array}\right) \text { d'après le lemme C.4.6. }
\end{aligned}
$$

L'exposant de $\mathbb{Z} / d \mathbb{Z}$ est donc bien $\sum_{x \mid \frac{n+1}{d}} \mu(x)\left(\begin{array}{c}g+\frac{n+1}{d x}-1 \\ g-1\end{array}\right)$.

\section{$\S$ C.4.d. Sous-groupes de $\mathbb{G}_{\mathrm{m}}^{g}$}

Lorsque $S$ est un schéma, un $S$-schéma en groupes est dit diagonalisable s'il est isomorphe à $D_{S}(M):=(\operatorname{Spec} \mathbb{Z}[M]) \times_{\mathrm{Spec} \mathbb{Z}} S$, où $M$ est un groupe abélien et $\mathbb{Z}[M]$ est la $\mathbb{Z}$-algèbre engendrée par $M$. Le schéma $D_{S}(M)$ est un schéma en groupes ; en effet, si $X$ est un $S$-schéma, on a

$$
\begin{aligned}
D_{S}(M)(X) & =\operatorname{Hom}_{\text {groupes }}\left(M, \Gamma\left(X, \mathcal{O}_{X}\right)^{*}\right) \\
& =\operatorname{Hom}_{\text {groupes }}\left(M, \mathbb{G}_{\mathrm{m}}(X)\right)
\end{aligned}
$$

Lorsque $M$ est le quotient de $\mathbb{Z}^{g}$ par un sous-groupe $\Phi$, on retrouve les sous-groupes usuels (en transcendance) de $\mathbb{G}_{\mathrm{m}}^{g}$ :

$D_{\mathbb{Z}}\left(\mathbb{Z}^{g} / \Phi\right)(X) \simeq \mathbf{T}_{\Phi}(X):=\left\{\left(x_{1}, \ldots, x_{g}\right) \in\left(\Gamma\left(X, \mathcal{O}_{X}\right)^{*}\right)^{g} ; x_{1}^{m_{1}} \cdots x_{g}^{m_{g}}=1 \quad \forall\left(m_{1}, \ldots, m_{g}\right) \in \Phi\right\}$.

Pour un schéma en groupes diagonalisable (dont les archétypes sont $\mathbb{G}_{\mathrm{m}}$ et le groupe des racines $n^{\text {èmes }}$ de l'unité) on a le résultat suivant ( $c f$. [42], page 28) :

Proposition C.4.8. Si $M$ est un groupe abélien, le schéma en groupes diagonalisable

$$
D_{S}(M):=\operatorname{Spec} \mathcal{O}_{S}[M]
$$

est lisse sur $S$ si et seulement si aucune caractéristique résiduelle de $M$ ne divise la torsion de $M$.

\section{Corollaire C.4.9.}

1. Le groupe multiplicatif $\mathbb{G}_{\mathrm{m}, S}:=D_{S}(\mathbb{Z})$ est lisse sur $S$.

2. Un sous-schéma en groupes $\mathbb{G}^{\prime}$ de $\mathbb{G}_{\mathrm{m} / \mathbb{Z}}^{g}$, défini par un sous groupe $\Phi$ de $\mathbb{Z}^{g}$ (i.e. $\mathbb{G}^{\prime}=$ $\left.D_{\mathbb{Z}}\left(\mathbb{Z}^{g} / \Phi\right)\right)$ est lisse sur $\operatorname{Spec} \mathbb{Z}$ si et seulement si une des conditions suivantes (équivalentes) est vérifiée :

(a) Le schéma en groupes $\mathbb{G}^{\prime}$ est connexe.

(b) Le groupe $\mathbb{Z}^{g} / \Phi$ est sans torsion* .

(c) Les mineurs d'ordre $\operatorname{rg}(\Phi)$ de la matrice associée à $\Phi$ sont premiers entre eux dans leur ensemble.

(d) L'idéal engendré par les mineurs précédents est l'anneau $\mathbb{Z}$.

\footnotetext{
${ }^{*}$ Le sous-groupe $\Phi$ est alors dit primitif.
} 
3. Le schéma en groupes des racines $n^{\text {èmes }}$ de l'unité (i.e. $D(\mathbb{Z} / n \mathbb{Z})$ ) n'est pas lisse sur $\mathbb{Z}$ (si $n>1)$ mais est lisse (et même étale) sur tout anneau $R$ dans lequel $n$ est inversible.

En particulier, si $\mathcal{W}$ est un hyperplan de l'espace tangent $t_{\mathbb{G}_{\mathrm{m}}^{g} / \mathbb{Z}}$ de la forme (dans la base habituelle) $a_{1} z_{1}+\cdots+a_{g} z_{g}=0$ avec les $a_{i}$ des entiers premiers entre eux * alors $\mathcal{W}$ est l'espace tangent du sous-groupe lisse de $\mathbb{G}_{\mathrm{m} / \mathbb{Z}}^{g}$, provenant de $\Phi=\mathbb{Z} .\left(a_{1}, \ldots, a_{g}\right)$.

\section{§ C.4.e. Translations}

Soit $T$ un $S$-schéma et $\pi: \mathbb{G} \rightarrow S$ un schéma en groupes de loi de groupe $m: \mathbb{G} \times \mathbb{G} \rightarrow \mathbb{G}$. Soit $g \in \mathbb{G}(T)$ et $g_{T}=\left(g, i d_{T}\right)$ l'élément de $\mathbb{G}_{T}(T)$ qui s'en déduit.

Définition C.4.10. Avec les notations précédentes, le morphisme de translation à gauche par $g$ est celui obtenu par composition :

$$
\tau_{g}: \mathbb{G}_{T} \stackrel{\sim}{\longrightarrow} T \times_{T} \mathbb{G}_{T} \stackrel{g_{T} \times i d_{T}}{\longrightarrow} \mathbb{G}_{T} \times \mathbb{G}_{T} \stackrel{m_{T}}{\longrightarrow} \mathbb{G}_{T} .
$$

De la même façon, le morphisme de translation à droite par $g$ est :

$$
\tau_{g}^{\prime}: \mathbb{G}_{T} \stackrel{\sim}{\longrightarrow} \mathbb{G}_{T} \times_{T} T \stackrel{i d_{T} \times g_{T}}{\longrightarrow} \mathbb{G}_{T} \times \mathbb{G}_{T} \stackrel{m_{T}}{\longrightarrow} \mathbb{G}_{T} .
$$

Ces morphismes de translation (à gauche et à droite) sont des isomorphismes (étales) de schémas. En particulier l'espace tangent $t_{\mathbb{G}, Q}=\operatorname{Hom}_{\mathcal{O}_{S}}\left(Q^{*} \Omega_{\mathbb{G} / S}^{1}, \mathcal{O}_{S}\right)$ de $\mathbb{G}$ en un point $Q \in \mathbb{G}(S)$ est isomorphe à l'espace tangent $t_{\mathbb{G} / S}$ de $\mathbb{G}$ le long de la section nulle $\varepsilon \in \mathbb{G}(S)$.

Exemple C.4.11. Soit $g \in \mathbb{G}_{\mathrm{a}}(T)=\Gamma\left(T, \mathcal{O}_{T}\right)$. La translation $\tau_{g}: \mathbb{G}_{\mathrm{a} / T} \rightarrow \mathbb{G}_{\mathrm{a} / T}$ est l'application dont le comorphisme est

$$
\begin{aligned}
\Gamma\left(T, \mathcal{O}_{T}\right)[X] & \longrightarrow \Gamma\left(T, \mathcal{O}_{T}\right)[X] \\
X & \longmapsto X+g
\end{aligned}
$$

Exemple C.4.12. Soit $g \in \mathbb{G}_{\mathrm{m}}(T)=\Gamma\left(T, \mathcal{O}_{T}\right)^{*}$. La translation $\tau_{g}: \mathbb{G}_{\mathrm{m} / T} \rightarrow \mathbb{G}_{\mathrm{m} / T}$ est l'application dont le comorphisme est

$$
\begin{aligned}
\Gamma\left(T, \mathcal{O}_{T}\right)\left[X, X^{-1}\right] & \longrightarrow \Gamma\left(T, \mathcal{O}_{T}\right)\left[X, X^{-1}\right] \\
X & \longmapsto g X
\end{aligned}
$$

À partir de cette définition, on obtient la notion de « forme différentielle sur $\mathbb{G}$ invariante à gauche » : une section globale $\omega$ de $\Omega_{\mathbb{G} / S}^{1}$ (ou plus généralement de $\wedge^{i} \Omega_{\mathbb{G} / S}^{1}$ ) est invariante à gauche si, pour tout $S$-schéma $T$, pour tout $g \in \mathbb{G}(T)$, on a $\tau_{g}^{*} \omega_{T}=\omega_{T}$ où $\omega_{T}$ est l'image inverse par la projection $\mathbb{G}_{T} \rightarrow \mathbb{G}$ de $\omega$. Le cotangent $\varepsilon^{*} \Omega_{\mathbb{G} / S}^{1}$ de $\mathbb{G}$ est le « lieu de naissance »des formes différentielles invariantes :

Proposition C.4.13. Soit $\varepsilon: S \rightarrow \mathbb{G}$ la section nulle de $\mathbb{G}$. Alors, pour tout $\omega_{0} \in \mathrm{H}^{0}\left(S, \varepsilon^{*} \Omega_{\mathbb{G} / S}^{1}\right)$, il existe une unique forme différentielle invariante à gauche $\omega \in \mathrm{H}^{0}\left(\mathbb{G}, \Omega_{\mathbb{G} / S}^{1}\right)$ telle que $\omega_{0}=\varepsilon^{*} \omega$ dans $\varepsilon^{*} \Omega_{\mathbb{G} / S}^{1}$.

*Cette hypothèse n'est pas automatiquement vérifiée car on est sur les $\mathbb{Z}$-modules. 


\section{$\S$ C.4.f. Multiplication par $n$}

Proposition C.4.14. Soit $\mathbb{G}$ un $S$-schéma en groupes commutatif lisse et de type fini. Soit $n \in \mathbb{N} \backslash\{0\}$. Si, pour tout $s \in S$, la caractéristique de $k(s)$ ne divise pas $n$ alors la multiplication par $n,[n]: \mathbb{G} \rightarrow \mathbb{G}$, est un morphisme étale.

Démonstration. cf. lemme $2 \mathrm{du} \S 7.3$ de [14].

\section{$\S$ C.5. Dérivations et opérateurs différentiels}

Ce paragraphe est extrait de EGA IV [43], § 16. Il vise à rappeler que la notion de lissité traduit l'existence d'une « excellente » famille d'opérateurs différentiels sur $\mathcal{O}_{X}$ (les dérivées « divisées », théorème C.5.6 ci-dessous).

\section{$\S$ C.5.a. Dérivations}

Définition C.5.1. Soit $f: X \rightarrow S$ un morphisme d'espaces annelés. Pour tout $\mathcal{O}_{X}$-module $\mathcal{F}$, on appelle $S$-dérivation de $\mathcal{O}_{X}$ dans $\mathcal{F}$ tout homomorphisme de faisceaux de groupes additifs $D: \mathcal{O}_{X} \rightarrow \mathcal{F}$ vérifiant les conditions suivantes :

a) pour tout ouvert $V$ de $X$, et tout couple de sections $\left(t_{1}, t_{2}\right)$ de $\mathcal{O}_{X}$ au-dessus de $V$, on a $D\left(t_{1} t_{2}\right)=t_{1} D\left(t_{2}\right)+D\left(t_{1}\right) t_{2}$.

b) pour tout ouvert $V$ de $X$, toute section $t$ de $\mathcal{O}_{X}$ au-dessus de $V$ et toute section $s$ de $\mathcal{O}_{S}$ au-dessus d'un ouvert $U$ de $S$ tel que $V \subseteq f^{-1}(U)$ on a $D\left(s_{\mid V} t\right)=s_{\mid V} D(t)$.

L'ensemble des $S$-dérivations de $\mathcal{O}_{X}$ dans $\mathcal{F}$ forment un $\Gamma\left(X, \mathcal{O}_{X}\right)$-module noté $\operatorname{Dér}_{S}\left(\mathcal{O}_{X}, \mathcal{F}\right)$.

Proposition C.5.2. Soit $f: X \rightarrow S$ un morphisme de schémas.

i) La différentielle $d_{X / S}: \mathcal{O}_{X} \rightarrow \Omega_{X / S}^{1}$ est une S-dérivation.

ii) Pour tout $\mathcal{O}_{X}$-module $\mathcal{F}$, l'application $u \rightarrow u \circ d_{X / S}$ induit un isomorphisme de$\Gamma\left(X, \mathcal{O}_{X}\right)$-modules

$$
\operatorname{Hom}_{\mathcal{O}_{X}}\left(\Omega_{X / S}^{1}, \mathcal{F}\right) \stackrel{\sim}{\longrightarrow} \operatorname{Dér}_{S}\left(\mathcal{O}_{X}, \mathcal{F}\right) .
$$

iii) On a également un isomorphisme de $\mathcal{O}_{X}$-modules*

$$
\mathcal{H o m}_{\mathcal{O}_{X}}\left(\Omega_{X / S}^{1}, \mathcal{F}\right) \stackrel{\sim}{\rightarrow} \mathcal{D e ́ r}_{S}\left(\mathcal{O}_{X}, \mathcal{F}\right) .
$$

On peut comprendre (intuitivement) cet isomorphisme en regardant ce qui se passe lorsque $X$ est le spectre premier d'un anneau de polynômes à $g$ variables (i.e. $X=\mathbb{A}_{\mathbb{Z}}^{g}$ ) et $\mathcal{F}$ est un $\mathcal{O}_{X}$-module quasi-cohérent provenant d'un module $M$. L'isomorphisme (C.12) est l'application

$$
\begin{aligned}
\operatorname{Hom}_{\mathbb{Z}[\mathbf{X}]}\left(\sum_{i=1}^{g} \mathbb{Z}[\mathbf{X}] . \mathrm{d} X_{i},\right. & M) \longrightarrow \operatorname{Dér}_{\mathbb{Z}}\left(\mathbb{Z}\left[X_{1}, \ldots, X_{g}\right], M\right) \\
\varphi & \longmapsto\left(P \mapsto \sum_{i=1}^{g} \frac{\partial P}{\partial X_{i}}(\mathbf{X}) \cdot \varphi\left(\mathrm{d} X_{i}\right)\right)
\end{aligned}
$$

C'est la dualité différentielle-dérivée!

\section{Propriétés.}

${ }^{*}$ On note en caractères romains le foncteur covariant de la catégorie des $\mathcal{O}_{X}$-modules dans celle des groupes abéliens et en caractères penchés le foncteur de la catégorie des $\mathcal{O}_{X}$-modules dans elle-même. 
1) Si $\pi: X \rightarrow S$ est localement de présentation finie et si $\mathcal{F}$ est un $\mathcal{O}_{X}$-module quasicohérent alors $\mathcal{D}$ ér ${ }_{S}\left(\mathcal{O}_{X}, \mathcal{F}\right)$ est un $\mathcal{O}_{X}$-module quasi-cohérent.

2) Si de plus $S$ est localement noethérien et si $\mathcal{F}$ est cohérent alors $\operatorname{Dér}_{S}\left(\mathcal{O}_{X}, \mathcal{F}\right)$ est $\mathcal{O}_{X}$-module cohérent.

\section{$\S$ C.5.b. Opérateurs différentiels}

Définition C.5.3. Soient $f: X \rightarrow S$ un morphisme de schémas, $\mathcal{F}, \mathcal{G}$ des $\mathcal{O}_{X}$-modules et $n$ un entier naturel. On dit qu'un homomorphisme de faisceaux de groupes additifs $D: \mathcal{F} \rightarrow \mathcal{G}$ est un opérateur différentiel d'ordre inférieur à $n$ relativement à $S$ s'il existe un homomorphisme de $\mathcal{O}_{X}$-modules $u: \mathcal{P}_{X / S}^{n} \otimes_{\mathcal{O}_{X}} \mathcal{F} \rightarrow \mathcal{G}$ tel que $D=u \circ d_{X / S}^{n}$.

L'ensemble des opérateurs d'ordre inférieur à $n$ de $\mathcal{O}_{F}$ dans $\mathcal{G}$ est un groupe additif, que l'on note $\operatorname{Diff}_{X / S}^{n}(\mathcal{F}, \mathcal{G})$, ou $\operatorname{Diff}_{X / S}^{n}$ lorsque $\mathcal{F}=\mathcal{G}=\mathcal{O}_{X}$. On note également $\operatorname{Diff}_{X / S}^{n}(\mathcal{F}, \mathcal{G})$ le préfaisceau de groupes additifs

$$
U \mapsto \operatorname{Diff}_{U / S}^{n}\left(\mathcal{F}_{\mid U}, \mathcal{G}_{\mid U}\right)
$$

Ce préfaisceau est un faisceau. Si $f: X \rightarrow S$ est un morphisme localement de présentation finie, $\mathcal{F}$ un $\mathcal{O}_{X}$-module de présentation finie et $\mathcal{G}$ un $\mathcal{O}_{X}$-module quasi-cohérent alors $\operatorname{Diff}_{X / S}^{n}(\mathcal{F}, \mathcal{G})$ est un $\mathcal{O}_{X}$-module quasi-cohérent.

Proposition C.5.4. Il y a un isomorphisme de faisceaux de groupes additifs

$$
\mathcal{H o m}_{\mathcal{O}_{X}}\left(\mathcal{P}_{X / S}^{n} \otimes \mathcal{F}, \mathcal{G}\right) \stackrel{\sim}{\longrightarrow} \operatorname{Diff}_{X / S}^{n}(\mathcal{F}, \mathcal{G})
$$

Proposition C.5.5. Soient $f: X \rightarrow S$ un morphisme de schémas, $\mathcal{F}, \mathcal{G}$ des $\mathcal{O}_{X}$-modules, $D: \mathcal{F} \rightarrow \mathcal{G}$ un homomorphisme de $f^{*} \mathcal{O}_{S}$-module, $n$ un entier naturel. Les conditions suivantes sont équivalentes :

a) $D$ est un opérateur différentiel d'ordre inférieur à $n$;

b) Pour toute section a de $\mathcal{O}_{X}$ au-dessus d'un ouvert $U$, l'homomorphisme $D_{a}: \mathcal{F}_{\mid U} \rightarrow \mathcal{G}_{\mid U}$ tel que, pour toute section $t$ de $\mathcal{F}$ au-dessus d'un ouvert $V \subseteq U$, on ait $D_{a}(t)=D(a t)-$ $a D(t)$, est un opérateur différentiel d'ordre $n-1$;

c) Pour tout ouvert $U$ de $X$, toute famille $\left(a_{i}\right)_{1 \leq i \leq n+1}$ de $n+1$ sections de $\mathcal{O}_{X}$ au-dessus de $U$, et toute section $t$ de $\mathcal{F}$ au-dessus de $U$, on a l'identité

$$
\sum_{H \subseteq\{1,2, \ldots, n+1\}}(-1)^{|H|}\left(\prod_{i \in H} a_{i}\right) D\left(\left(\prod_{i \notin H} a_{i}\right) t\right)=0 .
$$

L'avantage d'être sur un schéma différentiellement lisse provient du fait que l'on peut utiliser des « dérivées divisées ». En effet on a le

Théorème C.5.6. Soient $f: X \rightarrow S$ un morphisme, $U$ un ouvert de $X,\left(z_{\lambda}\right)_{\lambda \in \Lambda}$ une famille de sections de $\mathcal{O}_{X}$ au-dessus de $U$ telle que les $d z_{\lambda}$ forment un système de générateurs de $\Omega_{U / S}^{1}$. Les conditions suivantes sont équivalentes :

i) $f_{\mid U}$ est différentiellement lisse et $\left(d z_{\lambda}\right)$ est une base $d u \mathcal{O}_{U}$-module $\Omega_{U / S}^{1}$. 


\section{C.5. Dérivations et opérateurs différentiels}

ii) Il existe une famille $\left(D_{\underline{p}}\right)_{p \in \mathbb{N}(\Lambda)}$ d'opérateurs différentiels de $\mathcal{O}_{U}$ dans lui-même, vérifiant les conditions

$$
D_{\underline{p}}\left(z^{q}\right)=\left(\frac{q}{p}\right) z^{\underline{q}-\underline{p}} \text { pour } \underline{p}, \underline{q} \in \mathbb{N}^{(\Lambda)} .
$$

De plus lorsque ces conditions sont vérifiées, la famille $\left(D_{\underline{p}}\right)$ est déterminée de façon unique par les conditions précédentes et vérifie les relations :

$$
D_{\underline{p}} \circ D_{\underline{q}}=D_{\underline{q}} \circ D_{\underline{p}}=\frac{(\underline{p}+\underline{q}) !}{\underline{p} ! \underline{q} !} D_{\underline{p}+\underline{q}} .
$$

Enfin, si $\Lambda$ est fini, pour tout entier $n$, les $\left(D_{p}\right)_{|\underline{p}| \leq n}$ forment une base $d u \mathcal{O}_{U}$-module Diff ${ }_{U / S}^{n}$, autrement dit, tout opérateur différentiel d'ord̄e inférieur à $n$ sur $U$ s'écrit d'une seule façon sous la forme

$$
D=\sum_{|\underline{p}| \leq n} a_{\underline{p}} D_{\underline{p}}
$$

où les $a_{\underline{p}}$ sont des sections de $\mathcal{O}_{X}$ au-dessus de $U$. Lorsque $X$ est un schéma de caractéristique zéro,

$$
D_{\underline{p}}=(\underline{p} !)^{-1} \prod_{\lambda \in \Lambda} D_{\lambda}^{p_{\lambda}}
$$

où les $D_{\lambda}=\frac{\partial}{\partial z_{\lambda}}$ sont les formes coordonnées correspondant à la base $\left(d z_{\lambda}\right)$. 
Annexe C. Aide-mémoire sur la notion de voisinage infinitésimal 


\section{Références Bibliographiques}



[1] M. ABLy. Formes linéaires de logarithmes de points algébriques sur une courbe elliptique de type cm. Ann. Inst. Fourier (Grenoble), 50(1) :1-33, 2000.

[2] A. BAKER. Linear forms in the logarithms of algebraic numbers. i,ii,iii. Mathematika, 13/14 :204-216/102-107/220-228, 1966/1967.

[3] A. BAKER. A sharpening of the bounds for linear forms in logarithms i. ii. iii. Acta Arith., 21/24/27 :117-129/33-36/247-252, 1972/1973/1975.

[4] J. Bertin, J.-P. Demailly, L. Illusie, and C. Peters. Introduction à la théorie de Hodge, volume 3 of Panoramas et Synthèses. Soc. Math. France, 1996.

[5] D. BerTRAnd. Galois representations and transcendental numbers. In New advances in Transcendence Theory. Cambridge University Press, 1988.

[6] D. Bertrand. Minimal heights and polarizations on group varieties. Duke Math. J., 80(1) :223-250, 1995.

[7] D. Bertrand. Duality on tori and multiplicative dependence relations. J. Austral. Math. Soc., 62(Series A) :198-216, 1997.

[8] D. Bertrand and P. Philippon. Sous-groupes algébriques de groupes algébriques commutatifs. Illinois J. Math, 23 :263-280, 1988.

[9] A. BiJlsma. Simultaneous approximations in transcendental number theory . PhD thesis, Amsterdam : Mathematisch Centrum, 1978. Math. Centre Tracts. 94.

[10] A. Bijlsma and P.L. Cijsouw. Degree-free bounds for dependence relations. J. Austral. Math. Soc., 31(A) :496-507, 1981.

[11] Y. Bilu, G. Hanrot, and P.M. Voutier. Existence of primitive divisors of lucas and lehmer numbers. J. reine angew. Math., 539 :75-122, 2001.

[12] C. Birkenhake and H. LAnge. Complex abelian varieties, volume 302 of Grundlehren Der Mathematischen Wissenschaften. Springer-Verlag Berlin, 1992.

[13] E. Bombieri and J. VAaler. On siegel's lemma. Invent. Math., 73 :11-32, 1983. Addendum 75, 377 (1984).

[14] S. Bosch, W. LÜtkebohmert, and M. Raynaud. Néron Models, volume 21 of Ergebnisse der Mathematik und ihrer Grenzgebiete. Springer-Verlag Berlin, 1990.

[15] V. Bosser. Minorations de formes linéaires de logarithmes pour les modules de drinfeld. J. Number Theory, 75 :279-323, 1999.

[16] J.-B. Bost. Théorie de l'intersection et théorème de Riemann-Roch arithmétiques. In Séminaire Bourbaki. 1990/1991, volume 201-203 of Astérisque, pages 43-88. Société Mathématique de France. 
[17] J.-B. Bost. Arakelov geometry of abelian varieties. In Conference on Arithmetical Geometry, volume 96-51, Max Planck Institut für Mathematik Bonn, mars 1996. Notes manuscrites.

[18] J.-B. Bost. Intrinsic heights of stable varieties. Application to abelian varieties. Duke Math. J., 82 :21-70, 1996.

[19] J.-B. Bost. Périodes et isogénies des variétés abéliennes sur les corps de nombres (d'après D. Masser et G. Wüstholz). In Séminaire Bourbaki, volume 237 of Astérisque, pages 115161. Société Mathématique de France, 1996.

[20] J.-B. Bost. Cours de dea, 1997 et 1999. Notes manuscrites.

[21] J.-B. Bost. Potential theory and lefschetz theorems for arithmetic surfaces. Ann. Scient. Éc. norm. Sup, 32 (4 ème série) :241-312, 1999.

[22] J.-B. Bost. Algebraic leaves of algebraic foliations over number fields. Publications Mathématiques de l'IHÉS, 93 :161-221, 2001.

[23] J.-B. Bost, C. Soulé, and H. Gillet. Heights of projective varieties and positive Green forms. J. Amer. Math. Soc., 7(4) :903-1027, 1994.

[24] N. Bourbaki. Espaces Vectoriels Topologiques. Éléments de Mathématique. MassonParis, 1981.

[25] S. Bruiltet. D'une mesure d'approximation simultanée à une mesure d'irrationalité : le cas de $\gamma(1 / 4)$ et $\gamma(1 / 3)$. Prépublication, 1999.

[26] Y. Bugeaud. On the diophantine equation $x^{2}-p^{m}= \pm y^{n}$. Acta Arith., 80(3) :213-223, 1997.

[27] D.V. Chudnovsky and G.V.Chudnovsky. Padé approximations and diophantine geometry. In Proc. Natl. Acad. Sci. USA, volume 82, pages 2212-2216, 1985.

[28] G.V. ChudNovsky. Measures of irrationality, transcendence and algebraic independence. In Recent progress in Number Theory, pages 11-82. Cambridge Univ. Press., 1980.

[29] G.V. Chudnovsky. Contributions to the theory of transcendental numbers, volume XI. Providence, R.I. : American Math. Soc., 1984. Mathematical Surveys and Monographs.

[30] S. DAVID. Minorations de formes linéaires de logarithmes elliptiques, volume 62 of Mémoire de la Société Mathématique de France. S. M. F., 1995.

[31] S. DAvid and N. Hirata-Kohno. Recent progress on linear forms in elliptic logarithms. À paraître.

[32] S. DAVID and P. Philippon. Minorations des hauteurs normalisées des sous-variétés de variétés abéliennes. In V.K. Murty et M. Waldschmidt, editor, Number Theory, Tiruchirapalli, India, 3-6 janvier 1996, volume 210 of Contemp. Math., pages 333-364, 1998.

[33] S. DAVID and P. PhILIPPON. Minorations des hauteurs normalisées des sous-variétés de variétés abéliennes ii. Preprint, 2001.

[34] G. Diaz. Minorations de combinaisons linéaires non homogènes pour un logarithme elliptique. C. R. Acad. Sci, I 318(10) :879-883, 1994. «Tapuscrit » de la démonstration détaillée.

[35] N.I. Fel'DMAn and Yu.V. Nesterenko. Transcendental numbers, volume 44 of Encyclopaedia of Mathematical Sciences. Springer Berlin, 1998. 
[36] J.-M. Fontaine. Il n'y a pas de variété abélienne sur $\mathbb{Z}$. Invent. Math., 81 :515-538, 1985.

[37] G. Gillet and C. SoulÉ. An Arithmetic Riemann-Roch Theorem. Invent. Math., $110: 473-543,1992$.

[38] P. Graftieaux. Groupes formels et critères d'isogénie. Thèse de Doctorat, Université Paris VI, mars 1998.

[39] P. Graftienux. Formal Groups and Isogeny Theorem. Duke Math. J., 106 :81-121, 2001.

[40] P. Graftieaux. Formal subgroups of abelian varieties. Invent. Math., 145 :1-17, 2001.

[41] P. Griffiths and J. HARRIS. Principles of algebraic geometry. Pure and applied mathematics. Wiley, 1978.

[42] A. Grothendieck. Schémas en groupes I. Propriétés générales des schémas en groupes, volume 151 of L. N. M. Springer-Verlag, 1970.

[43] A. Grothendieck and J. Dieudonné. Éléments de Géométrie Algébrique, volume IV. I.H.E.S, 1967. Étude locale des Schémas et des Morphismes de Schémas.

[44] M. Hazewinkel. Formal Groups and Applications. Pure and Applied Mathematics. Academic Press, 1978.

[45] N. Hirata-Kohno. Formes linéaires de logarithmes de points algébriques sur les groupes algébriques. Invent. Math., 104 :401-433, 1991.

[46] N. Hirata-Kohno. Approximations simultanées sur les groupes algébriques commutatifs. Compos. Math., 86 :69-96, 1993.

[47] G. Kempf. Complex Abelian Varieties and Theta Functions. Springer Verlag Berlin, 1991.

[48] J.-P. LAFOn. Algèbre commutative, volume 24 of Collection enseignement des sciences . Hermann, 1977. Langages géométrique et algébrique.

[49] S. LAng. Diophantine approximations on toruses. Amer. J. Math., 86 :521-533, 1964.

[50] S. LANG. Transcendental numbers and diophantine approximations. Bull. Amer. Math. Soc., 77 :635-677, 1971.

[51] M. Laurent. Hauteur de matrices d'interpolation. In Approximations Diophantiennes et Nombres Transcendants, Luminy 1990, pages 215-238. de Gruyter, 1990.

[52] M. Laurent, M. Mignotte, and Y. Nesterenko. Formes linéaires en deux logarithmes et déterminants d'interpolation. J. Number Theory, 55(2) :285-321, 1995.

[53] M. LE. On the diophantine equation $d_{1} x^{4}-d_{2} y^{2}=1$. Acta Arith., 76(1) :1-9, 1996.

[54] F. Lemmermeyer, M. Mignotte, and A. Pethoe. On the family of thue equations $x^{3}-(n-1) x^{2} y-(n+2) x y^{2}-y^{3}=k$. Acta Arith., 76(3) :245-269, 1996.

[55] J.H. Loxton and A.J. van der Poorten. Multiplicative dependence in number fields. Acta Arith., 42 :291-302, 1983.

[56] H. Matsumura. Commutative ring theory. Cambridge University Press, 1989.

[57] M. Mignotte. Sur l'équation $x^{p}-y^{q}=1$ lorsque $p \equiv 5 \bmod 8$. C. R. Math. Acad. Sci., Soc. R. Can, 18(5) :228-322, 1996. 
[58] M. MignotTe and Y. Roy. Catalan's equation has no new solution with either exponent less than 10651. Exp. Math., 4(4) :259-268, 1995.

[59] L. Moret-Bailly. Métriques permises. In Séminaire sur les pinceaux arithmétiques : La conjecture de Mordell, Astérisque. S. M. F., 1985. éditeur L. Szpiro.

[60] L. Moret-Bailly. Pinceaux de variétés abéliennes, volume 129 of Astérisque. Société Mathématique de France, 1985.

[61] L. Moret-Bailly. Sur l'équation fonctionnelle de la fonction thêta de riemann. Compositio Math., 75(2) :203-217, 1990.

[62] G. Nicklash and N.P. Smart. Exceptional units in a family of quartic number fields. Math. Comput., (222) :759-772, 1998.

[63] P. Philippon. Lemme de zéros dans les groupes algébriques commutatifs. Bull. Soc. Math. France, 114 :355-383, 1986. Errata et Addenda, id.115 (1987).

[64] P. Philippon. Nouveaux lemmes de zéros dans les groupes algébriques commutatifs. Rocky Mt. J. Math., 26(3) :1069-1088, 1996.

[65] P. Philippon and M. WAldschmidt. Formes linéaires de logarithmes sur les groupes algébriques commutatifs. Illinois J. Math., 32(2) :281-314, 1988.

[66] P. Philippon and M. Waldschmidt. Formes linéaires de logarithmes simultanées sur les groupes algébriques commutatifs. In Séminaire de Théorie des Nombres Paris 1986 - 87, volume 75 of Progress in Mathematics, pages 313-347. Birkhäuser Boston, Inc., 1989. Édité par Catherine Goldstein.

[67] H. RandRiambololona. Hauteurs de sous-schémas, géométrie d'Arakelov des schémas de Hilbert et exemples d'utilisation de méthodes arakeloviennes en théorie de l'approximation diophantienne. Thèse de doctorat, Université Paris XI, Janvier 2002.

[68] M. Raynaud. Modèles de néron. C. R. Acad. Sci Paris, A 162 :345-347, 1966.

[69] G. RÉmond. Sur des problèmes d'effectivité en géométrie diophantienne. Thèse de Doctorat, Université Paris VI, septembre 1997.

[70] D.J.S. Robinson. A Course in the Theory of Groups, volume 80 of Graduate Texts in Mathematics. Springer-Verlag, 1982.

[71] W.M. SchmidT. Diophantine Approximations and Diophantine Equations, volume 1467 of Lecture Notes in Math. Springer-Verlag, 1991.

[72] J.-P. SERRE. Représentations linéaires des groupes finis . Collection Méthodes. Hermann, 1994 edition, 1967.

[73] J.-P. SERRE. Quelques propriétés des groupes algébriques commutatifs, volume 69/70, pages 191-202. Astérisque, 1970.

[74] B.V. ShABAT. Distribution of Values of Holomorphic Mappings, volume 61 of Translations of Mathematical Monographs. American Mathematical Society, 1985.

[75] J.H. Silverman. Advanced Topics in the Arithmetics of elliptic Curves, volume 151 of G. T. M. Springer-Verlag, 1994.

[76] L. Szpiro. La conjecture de mordell [d'après g. faltings]. In Astérisque, volume 121/122, pages 83-103. S. M. F., 1985. 
[77] W.V. VAsconcelos. Computational methods in commutative algebra and algebraic geometry. With chapters by D. Eisenbud, D.R. Grayson, J. Herzog and M. Stillman, volume 2 of Algorithms and computation in Mathematics . Springer-Verlag Berlin Heidelberg, 1998.

[78] E. ViadA-Aenle. Elliptic Isogenies and Slopes . Dissertation, Swiss Federal Institute of technology Zurich, 2001. Number 14195.

[79] M. Waldschmidt. Nombres Transcendants, volume 402 of Lecture Notes in Mathematics. Springer-Verlag, 1974.

[80] M. WALDSChmidT. Nombres transcendants et groupes algébriques, volume 69/70 of Astérisque. Société Mathématique de France, 1979.

[81] M. Waldschmidt. A lower bound for linear forms in logarithms. Acta Arith, 37 :257$283,1980$.

[82] M. WALDschmidt. Nouvelles méthodes pour minorer des combinaisons linéaires de logarithmes de nombres algébriques. Séminaire de Théorie des Nombres de Bordeaux, Ser. II 3(1) :129-185, 1991.

[83] M. WaldschmidT. Approximation diophantienne dans les groupes algébriques commutatifs. (I) : Une version effective du théorème du sous-groupe algébrique. J. reine angew. Math, 493 :61-113, 1997.

[84] M. Waldschmidt. Diophantine Approximation On Linear Algebraic Groups, volume 326 of Grundlehren der mathematischen Wissenschaften. Springer, 2000.

[85] G. WÜstholz. Some remarks on a conjecture of waldschmidt. In Approximations diophantiennes et nombres transcendants (Luminy 1982), volume 31 of Prog. Math., pages 329-336, 1983.

[86] G. WÜstholz. Algebraische punkte auf analytischen untergruppen algebraischer gruppen. Annals of Math., 129(3) :501-517, 1989.

Université Jean-Monnet de Saint-Étienne

ÉQUiPE DE THÉORIE DES NOMBRES

23, rue du Docteur Paul Michelon

42023 Saint-Etienne Cedex 2

Eric.Gaudron@univ-st-etienne.fr 


\section{Résumé}

Cette thèse s'inscrit dans la lignée des travaux relatifs à la théorie des formes linéaires de logarithmes. Elle comporte deux parties ainsi que trois annexes.

Dans la première partie, nous nous intéressons au cas général d'un groupe algébrique commutatif quelconque, défini sur la clôture algébrique de $\mathbb{Q}$. Étant donné un tel groupe $G$, un hyperplan $W$ de l'espace tangent à l'origine de $G$ et $u$ un point complexe de cet espace tangent, dont l'image par l'exponentielle du groupe de Lie complexe $G(\mathbb{C})$ est algébrique, nous obtenons une minoration de la distance de $u$ à $W$, qui améliore les résultats connus auparavant et qui, en particulier, est optimale en la hauteur de l'hyperplan $W$. La démonstration repose sur la méthode de Baker ainsi que sur un nouvel argument de nature arithmétique (procédé de changement de variables de Chudnovsky) qui nous permet d'évaluer précisément les normes ultramétriques des nombres algébriques construits au cours de la preuve.

Dans la seconde partie, nous étudions plus en détail le « cas abélien non-homogène » (dans lequel le groupe $G$ est le produit direct du groupe $\mathbb{G}_{\mathrm{a}}$ et d'une variété abélienne) et nous établissons une nouvelle mesure, comparable à celle donnée dans la première partie mais totalement explicite en les invariants liés à la variété abélienne. La particularité de cette seconde partie est de mettre en œuvre, pour la première fois dans ce contexte, la méthode des pentes de J.-B. Bost et certains résultats de géométrie d'Arakelov qui lui sont attachés.

Mots-clés: Formes linéaires de logarithmes, groupe algébrique, méthode de Baker, groupe formel, logarithme formel, méthode des pentes, géométrie d'Arakelov.

\section{Abstract}

This thesis falls within the theory of linear forms in logarithms. It comprises two parts as well as three annexes.

In the first part, we are interested in the general case of an unspecified algebraic commutative group, defined over the algebraic closure of $\mathbb{Q}$. Given such a group $G$, an hyperplane $W$ of the tangent space at the origin of $G$ and $u$ a complex point of this tangent space, whose image by the exponential map of the Lie group $G(\mathbb{C})$ is an algebraic point, we obtain a lower bound for the distance between $u$ and $W$, which improves the results known before and which is, in particular, the best possible for the height of the hyperplane $W$. The demonstration rests on Baker's method as well as a new arithmetic argument (Chudnovsky's process of variable change) which enables us to give a precise estimate of the ultrametric norms of some algebraic numbers, built during the proof.

In the second part, we study in details the "abelian non-homogeneous case" (in which the group $G$ is the direct product of $\mathbb{G}_{\mathrm{a}}$ by an abelian variety) and we establish a new measure, comparable with the one given in the first part, but totally explicit in function of the invariants of the abelian variety. An important feature of this second part is the implementation, for the first time in this context, of J.-B. Bost's slopes method and some results of Arakelov geometry naturally associated with it.

Keywords: Linear forms in logarithms, algebraic group, Baker's method, formal group, formal logarithm, slopes method, Arakelov Geometry.

MSC2000 : 11J86, 14L05, 14L10, 11J20, 14G40. 
